Florida International University

FIU Digital Commons

3-4-2019

\title{
Role of Withaferin A as a Neuroprotectant against Beta Amyloid Induced Toxicity and associated mechanism
}

\author{
Sneham Tiwari \\ Florida International University, stiwa004@fiu.edu
}

Follow this and additional works at: https://digitalcommons.fiu.edu/etd

Part of the Biology Commons, and the Molecular and Cellular Neuroscience Commons

\section{Recommended Citation}

Tiwari, Sneham, "Role of Withaferin A as a Neuroprotectant against Beta Amyloid Induced Toxicity and associated mechanism" (2019). FIU Electronic Theses and Dissertations. 3963.

https://digitalcommons.fiu.edu/etd/3963

This work is brought to you for free and open access by the University Graduate School at FIU Digital Commons. It has been accepted for inclusion in FIU Electronic Theses and Dissertations by an authorized administrator of FIU Digital Commons. For more information, please contact dcc@fiu.edu. 


\title{
FLORIDA INTERNATIONAL UNIVERSITY
}

\author{
Miami, Florida
}

\section{ROLE OF WITHAFERIN A AS A NEUROPROTECTANT AGAINST BETA AMYLOID INDUCED TOXICITY AND ASSOCIATED MECHANISM}

\author{
A dissertation submitted in partial fulfillment of \\ the requirements for the degree of \\ DOCTOR OF PHILOSOPHY \\ in \\ BIOMEDICAL SCIENCES \\ by \\ Sneham Tiwari
}




\section{To: Dean Robert Sackstein \\ Herbert Wertheim College of Medicine}

This dissertation, written by Sneham Tiwari, and entitled Role of Withaferin A as a Neuroprotectant against Beta Amyloid Induced Toxicity and Associated Mechanism, having been approved in respect to style and intellectual content, is referred to you for judgment.

We have read this dissertation and recommend that it be approved.

$\begin{array}{r}\hline \text { Nazira El-Hage } \\ \hline \text { Hoshang Unwalla } \\ \hline \text { Ajeet Kaushik } \\ \hline \text { Michal Toborek } \\ \hline \text { Chenzhong Li } \\ \hline \text { Madhavan Nair, Major Professor }\end{array}$

Date of Defense: March 4, 2019

The dissertation of Sneham Tiwari is approved.

Dean Robert Sackstein
Herbert Wertheim College of Medicine

Andres G. Gil Vice President for Research and Economic Development and Dean of the University Graduate School

Florida International University, 2019 


\section{DEDICATION}

This work is firstly dedicated to my late grandparents Shyama and Lalta Prasad Tiwari, whose dreams far exceeded those of my own, to my late uncle Prof. Suresh Chandra Tiwari, PhD for inspiring me to the field of

Science,

and

to my maternal grandparents Sushila and Gopal Mishra

for always motivating me

and

For my loving and supporting parents

Neelam Tiwari and Mahesh Tiwari 


\section{ACKNOWLEDGMENTS}

First and foremost, I would like to express my heartfelt gratitude to Prof, Madhavan Nair, my Dissertation Advisor and mentor, for providing me this opportunity, to pursue my $\mathrm{PhD}$ under his esteemed and brilliant guidance. I can't thank you enough Prof. Nair for your support and guidance, for giving me freedom to design and progress with the research project, for guiding me throughout, for teaching me the importance of independent thinking and providing me a strong foundation to grow as a student researcher. Your guidance is precious and none of this would have been possible without your constant supervision. I would also like to extend my gratitude towards my respected Dissertation committee members, Dr. Nazira El-Hage, Dr. Hoshang Unwalla, Dr. Ajeet Kaushik, Dr. Michal Toborek and Dr. Chenzhong Li for agreeing to be on my committee, and for providing their positive feedbacks, guidance and support. Thank you for being so patient and helping me positively.

I would like to express my sincere gratitude to the funding agencies, for supporting my PhD project. My studies were supported by The National Institute of Health (Grant\# R01DA034547), Florida Department of Health's Ed, and Ethel Moore Alzheimer's Disease Research Program (Grant\# 800009191) supported this work. I would like to acknowledge the University Graduate School, Florida International University, for awarding me the prestigious Dissertation Year Fellowship 2018 and supporting my PhD. I would especially like to express my gratitude to Dr. Alexander Agoulnik for always having an open door to provide guidance and support throughout. A special thank you to Ms. Odalys De La Rosa for helping me with all the requirements starting from the admission process to graduation. She has been so patient with me by always helping me with the paper work, signatures and numerous questions, always with a smile on her face. Research challenges become more interesting if we have a virtuous team to work with, and I feel so blessed to have an 
amazing team of colleagues, who supported and helped me through out. My special thanks are extended to the Immunology crew including Dr. Hiten Chand, Dr. Rahul Dev Jayant, Dr. Venkata Atluri, Dr. Asahi Tomitaka Dr. Andrea Raymond, Dr. Marisela Agudelo, Dr. Nagesh Kolishetti, Dr. Arti Vashist, Dr. Sudheesh Pilakka, Dr. Purnima Gupta, Dr. Roozbeh Nikkhah-Moshaie, Dr. Zaohua Huang, for their help and guidance in the laboratory experiments and constant support. A special thank you to Ms. Adriana Yndart for being a fantastic colleague and a wonderful friend and for helping me with flow cytometry experiments and analysis. Big thank you to Dr. Prem Chapagain, and Dr. Krati Sharma for helping me with the in-silico studies. I am extremely thankful to Dr. Hind Harrak, Karla Munoz, Kimberly Justin and Ariele Riviere for their immense help. I would also like to acknowledge my friends I earned in this journey, who were there to help me through thick and thins, a big shout-out to Dr. Jessica Lapierre, Mr. Kyle Martins, Dr. Myosotys Rodriguez, Yashaswi Tapadia, and Udaya Buchireddy, for being my family away from home. I acknowledge Prof. Nazira El-Hage for helping me with the microscopy experiment and allowing me to use the microscope, Prof. Hiten Chand for helping and allowing me to use Keyence Microscope, Dr. Marisela Agudelo and Tiyash Parira for helping and Dr. Agudelo for Single flow cytometry, Dr. Manuel Barbieri for the Flow cytometry and Dr. Jonathan Geiger for kindly gifting us the SH-APP cells.

There are some very important people to be acknowledged from my hom country India, because it would not have been possible without their support. A big thank you to my parents Mahesh and Neelam Tiwari and my brother Shivam Tiwari for being there for me throughout, even if they were so far, they were always just a phone/video call away. I would also want to give a special mention to my former mentor Dr. Shailendra Saxena, who supported me when I was working as a Project JRF at Centre for Cellular and Molecular Biology, India and urged me to dream bigger and aim for PhD in the United 
States. He has been a constant support eversince and I thank him with all my heart for his support. I want to thank rest of my very big family back in India for living this dream with me and praying for me.

Last and most important, I am extremely grateful to Herbert Wertheim College of Medicine at Florida International University, for accepting me as a student in the brilliant PhD in Biomedical Sciences program. It gives me immense happiness to be able to associate myself with this prestigious institution. As an International student, it is always a little more challenging to survive in a new country, but the ISSS office made my journey so smooth with their constant guidance and support, I am extremely thankful to the ISSS team, especially to Claudia Balzan for her help and guidance.

I acknowledge Dove Medical Press as the original publisher of the article titled "Alzheimer' disease: Pathogenesis, Diagnostics and Therapeutics.Accepted in the the International Journal of Nanomedicine, 2019. I also thank them for giving me the permission to include the article in the chapter in my thesis. Additionally, I also want to acknowledge Frontiers to allow me to include my article titled "Withaferin A Suppresses Beta Amyloid in APP Expressing Cells: Studies for Tat and Cocaine Associated Neurological Dysfunctions" doi: 10.3389/fnagi.2018.00291, in my thesis and for allowing me to use the article in the chapter in my thesis. 


\section{ABSTRACT OF THE DISSERTATION}

\section{ROLE OF WITHAFERIN A AS A NEUROPROTECTANT AGAINST BETA AMYLOID \\ INDUCED TOXICITY AND ASSOCIATED MECHANISM}

by

Sneham Tiwari

Florida International University, 2019

Miami, Florida

Professor Madhavan Nair, Major Professor

Neurological disorders are the biggest concern globally and ageing contributes in worsening the disease scenarios. In $A D$ or $A D$ like diseases, there is abnormal accumulation of extracellular amyloid beta produced due to abnormal processing of the transmembrane amyloid precursor protein, by $\beta$ and $y$-secretases. It spreads in the cortical and limbic regions of the brain leading to neuronal toxicity, impairment in memory and neurological functions. A $\beta$ deposition in the CNS is common in aging HIV patients. Neurotoxic protein Tat, results in increased $A \beta$ in combination with drugs of abuse cocaine. We examined the role of Withaferin $A$, against $A \beta$ induced neurotoxicity. Our invitro dose optimization study demonstrates that lower concentrations $(0.5-2 \mu \mathrm{M})$ of WA significantly reduce the $A \beta 40$, without inducing cytotoxicity in the APP plasmid transfected SH-SY5Y cells (SHAPP). We demonstrate that A $\beta$ secretion is increased in the presence of Tat $(50 \mathrm{ng} / \mathrm{ml})$ and $\operatorname{coc}(0.1 \mu \mathrm{M})$, WA reduces the Tat and coc induced increase in A $\mathrm{A} 40$. Additionally, we studied the role of WA against NF-kB mediated neuroinflammation, and observed that WA inhibits the expression of NFkB2 and RELA transcription factors, which play a major role in the expression of inflammatory chemokines. Further, to address the issue of minimal drug bioavailability in the CNS, we developed the WA loaded liposomal 
nanoformulation (WA-LNF) and characterized its size (499+/-50nm), toxicity and drug binding efficacy (28\%). Our in-vitro 3D BBB transmigration of WA-LNF demonstrated $\sim 40 \%$ transmigration efficiency. Furthermore, it was imperative for us to understand the mechanism of action of WA, therefore we studied the molecular mechanism of interaction of WA with $A \beta$ protein by in-silico molecular dynamics simulations. We demonstrated that WA binds to the middle region of $A \beta$ protein and the amino acid motif involved were FAEDVGS highlighting the mid-region $A \beta$ capture by WA. 3 Hydrogen bonds were formed between WA and the amino acids, ASN17, GLY15 and SER16. This study reports WA as a potent neuroprotectant against amyloid induced neurotoxicity. Our study may have an immense therapeutic potential to target $A \beta$ in the CNS, in the ageing patients and/or PLWH and/or ageing drug abusers. 


\section{TABLE OF CONTENTS}

CHAPTER

PAGE

CHAPTER 1: ALZHEIMER 'S DISEASE: PATHOGENESIS, DIAGNOSTICS

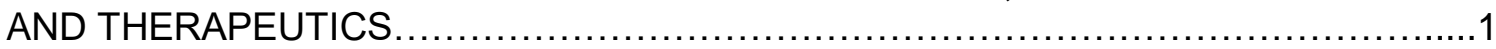

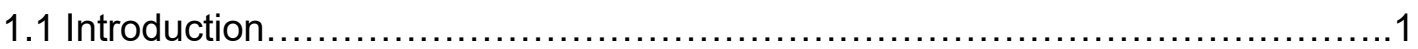

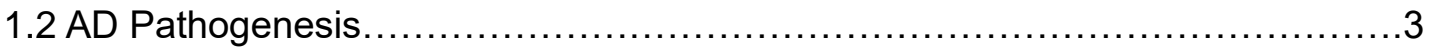

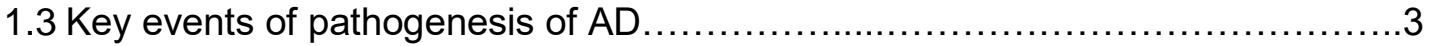

1.3.1 Structure and function of amyloid precursor protein...................... 4

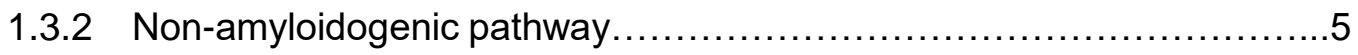

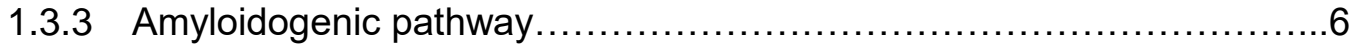

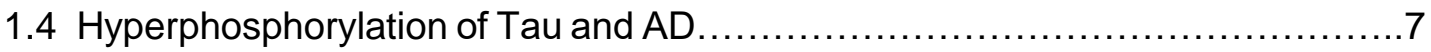

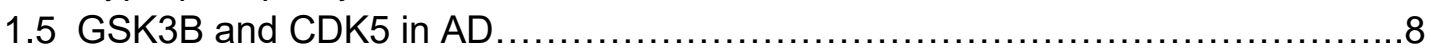

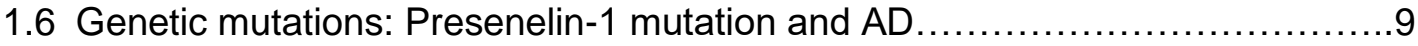

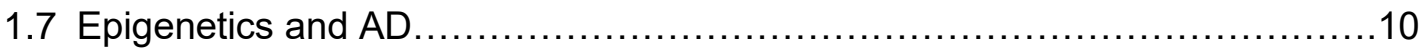

1.8 Microglial infiltration during plaque formation: leading to neurodegeneration....11

1.8.1 Microglia and $A \beta$ clearance ............................................... 14

$1.9 A \beta$ and HIV-1 associated neurological disorders................................. 14

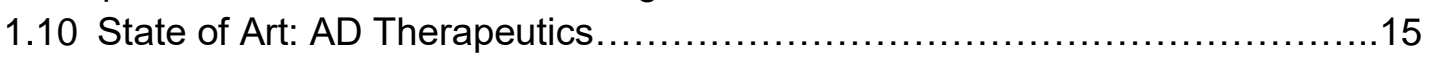

1.11 Diagnostics for AD: Labeling and imaging ......................................17

1.12 Need of nanotechnology as Therapeutic strategy ..............................19

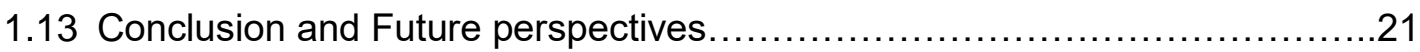

CHAPTER 2: WITHAFERIN A SUPPRESSES BETA AMYLOID IN APP EXPRESSING CELLS: STUDIES FOR TAT AND COCAINE ASSOCIATED NEUROLOGICAL DYSFUNCTIONS ...................................23

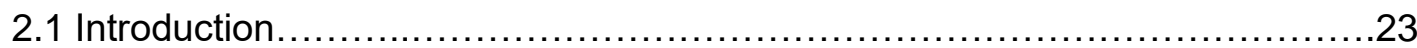

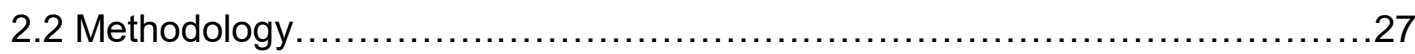

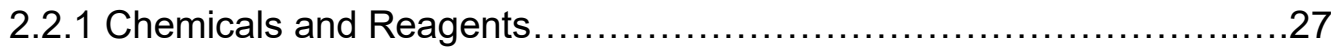

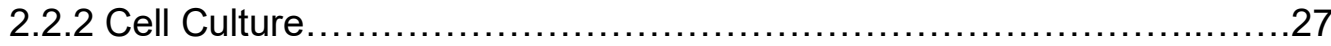

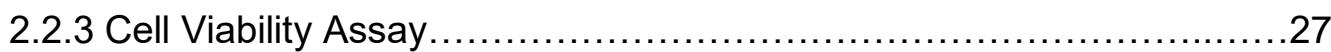

2.2.4 Tat and Coc Treatment of SH-APP Cells................................28

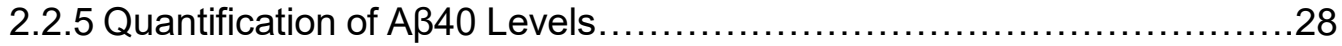

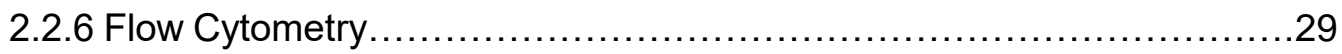

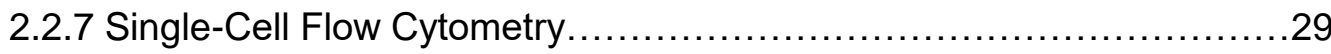

2.2.8 Immunofluorescence Staining and Analysis for Studying Beta Amyloid Aggregation...........................................................

2.2.9 Immunofluorescence Staining for Studying Effect of Withaferin A

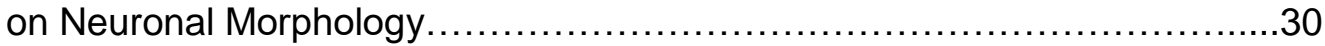

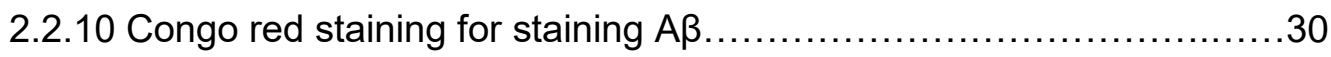

2.2.11 Beta secretases ELISA ......................................................

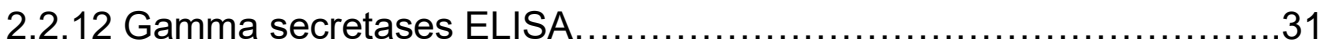




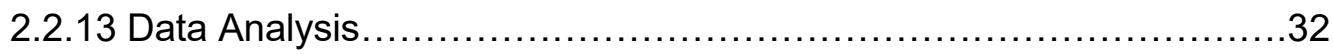

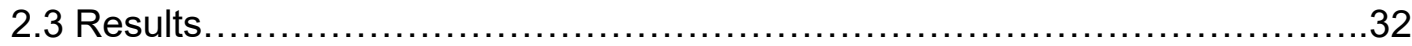

2.3.1 WA dose optimization and $A \beta$ decreasing efficacy studies in

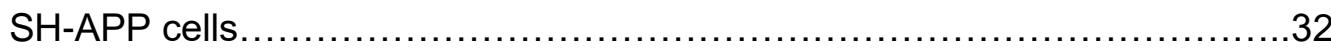

2.3.2 Effect of HIV-Tat protein on A $\beta$ production in SH-APP cells.................35

2.3.3 Effect of HIV-Tat protein and Cocaine on $A \beta$ production in

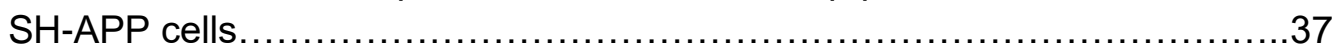

2.3.4 Tat and coc Induced Increase in A 340 Levels, in combination............38

2.3.5 WA reverses Tat and Cocaine induced Amyloid aggregates

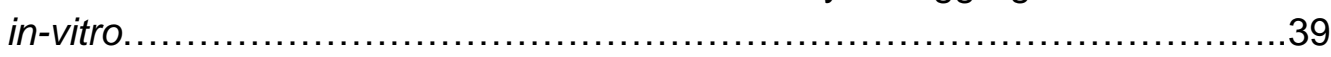

2.3.6 WA may decrease Cocaine induced neurotoxicity .........................41

2.3.7 Congo red stain based quantification of $A \beta$ in WA treated cells............44

2.3.8 WA effect on Gamma secretases levels......................................45

2.3.9 WA effect on Beta secretases levels.......................................46

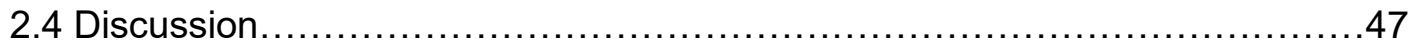

CHAPTER 3: WA AND CRID3 AGAINST A INDUCED INFLAMMATION ................52

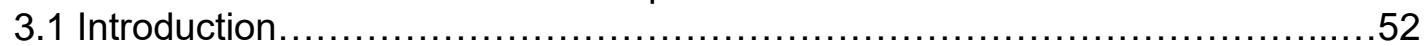

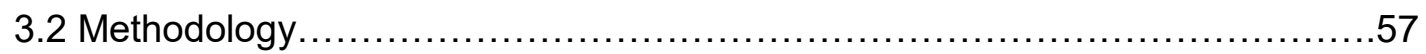

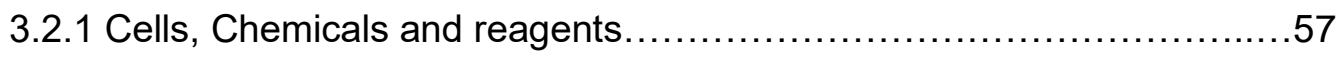

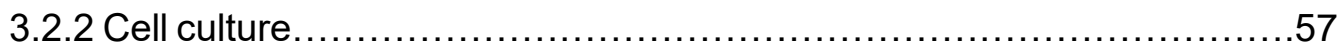

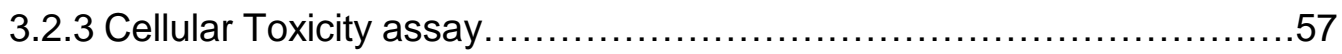

3.2.4 Treatment with CRID3 and estimation of Caspase-1 and

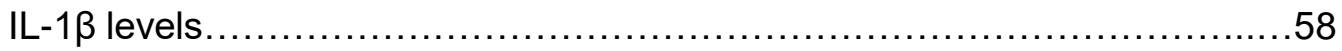

3.2.5 Estimation of NF-kB mediated neuroinflammatory genes on

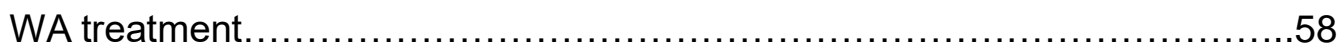

3.2.6 Estimation of inflammasome mediated genes expression on

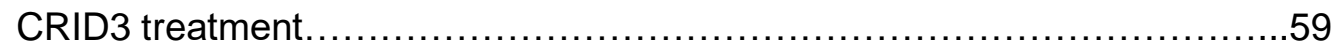

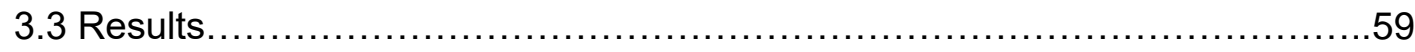

3.3.1 CRID3 and WA do not cause cellular toxicity .............................59

3.3.2 WA inhibits inflammatory regulated genes associated with

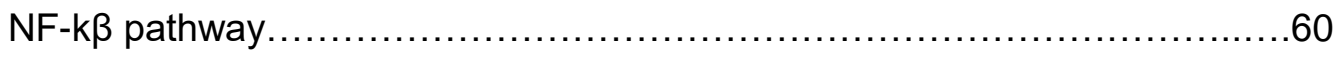

3.3.3 CRID3 inhibits inflammatory regulated genes associated with

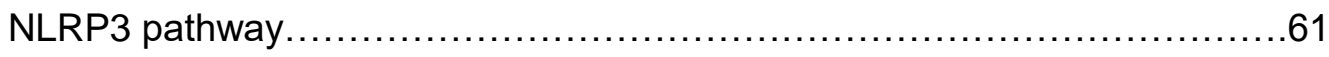

3.3.4 CRID3 inhibits Caspase- 1 and IL-1 $1 \beta$ protein expression...................63

3.3.5 Liposomal Nanoformulation of CRID3 ..................................65

3.3.6 Liposomal CRID3 and its transmigration across BBB....................67

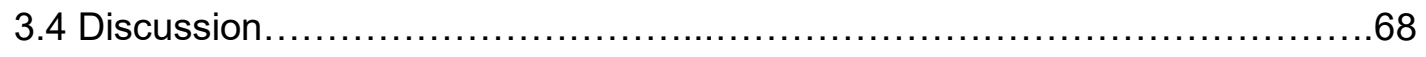

CHAPTER 4: LIPOSOMAL NANOFORMULATION OF WITHAFERIN A AS

A PROMISING NANOCARRIER AGAINST AMYLOID BETA ...........................76

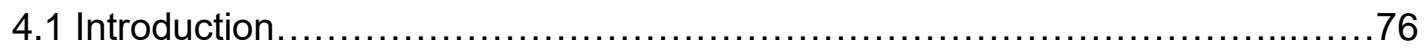

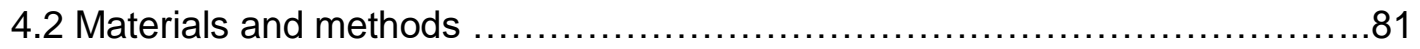




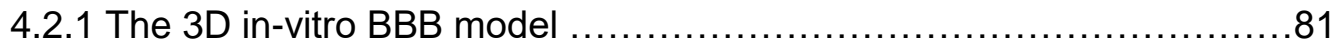

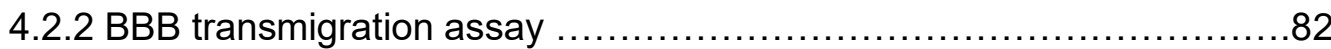

4.2.3 Synthesis of WA Liposomal Nanoformulation ......................... 83

4.2.4 Characterization of liposomes size and bound drug calculation...........85

4.2.5 Toxicity studies: Trypan Blue Cell viability test .......................85

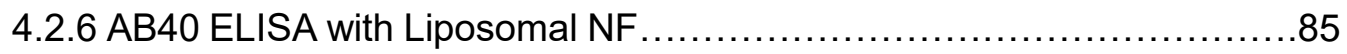

4.2.7 WA Drug release kinetics from the Liposome by Dialysis

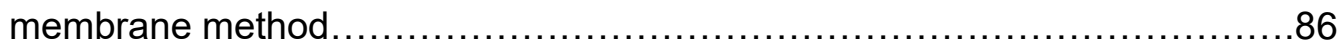

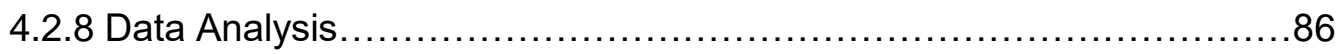

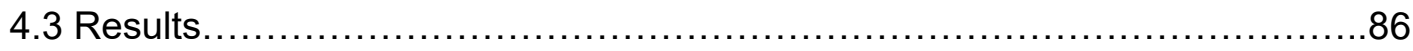

4.3.1 In-vitro 3D Blood Brain Barrier model ................................ 86

4.3.2 Development and characterization of NF ............................ 87

4.3.3 Liposomal Nanoformulations are not toxic ..........................90

4.3.4 Liposomal Nanoformulations transmigrate across the BBB.............91

4.3.5 Functional Efficacy of the WA-LNF upon BBB transmigration (ELISA)....91

4.3.6 WA release study by membrane dialysis method.....................92

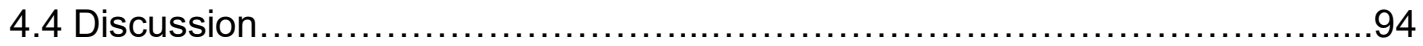

\section{CHAPTER 5: MOLECULAR BASIS FOR MID-REGION A $\beta$ CAPTURE BY}

LEADING ALZHEIMER'S DISEASE IMMUNOTHERAPIES:

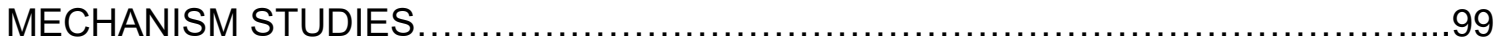

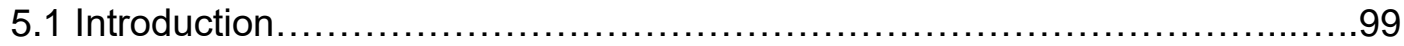

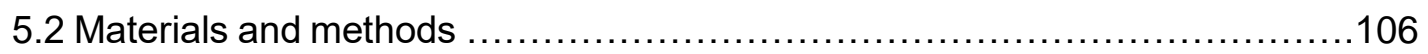

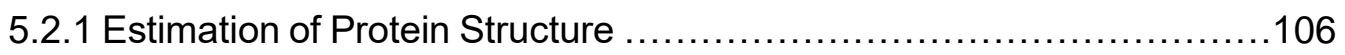

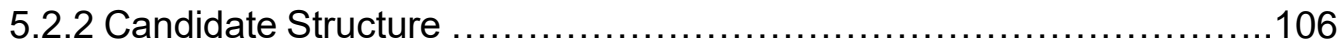

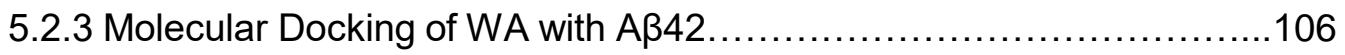

5.2.4 Molecular Dynamics Simulation....................................106

5.2.5 Protein Structure Visualization and analysis.........................107

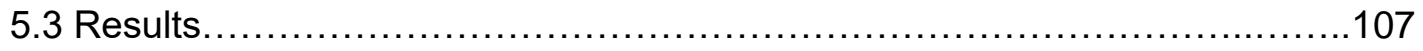

5.3.1 Physical and Chemical properties of WA compound...................108

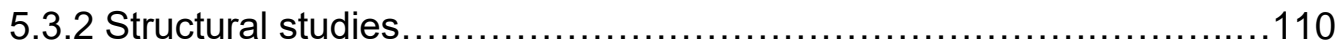

5.3.3 A $\beta$ has an interactive hydrophobic motif...........................112

5.3.4 Drug-protein interactions happens through Hydrogen bonding and the binding is stable and specific......................................... 114

5.3.5 Dynamic modelling: Speculating the mechanism of action of WA......116

5.3.6 Protein drug binding is stable and specific with time lapse............117

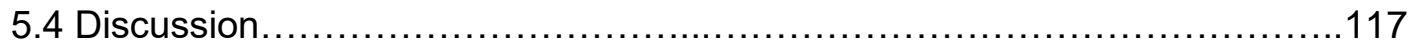

CHAPTER 6: FINAL CONCLUSIONS AND FUTURE DIRECTIONS $\ldots \ldots \ldots \ldots \ldots \ldots \ldots 122$

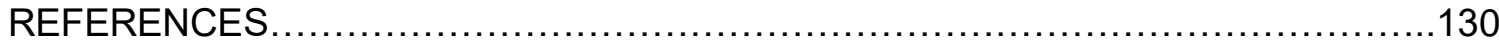

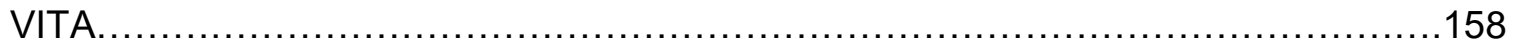




\section{LIST OF TABLES}

TABLE

PAGE

Table 1.1 Drugs specific to amyloid which are studied and target sufficiently against fundamental and proximate degenerative mechanisms.

Table 3.1 WA inhibits NF-k $\beta$ mediated inflammatory response related genes expression array

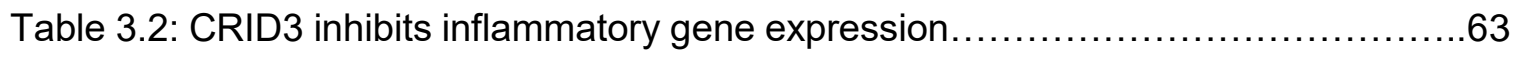

Table 4.1: Characterization of developed Liposomal Nanoformulation..................89

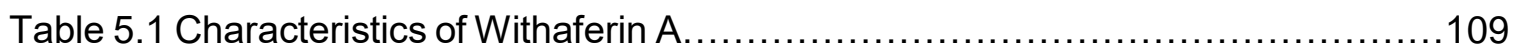




\section{LIST OF FIGURES}

FIGURE

PAGE

Figure 1.1 An overview of the fields of research that need to be elucidated to understand the pathophysiology of $A D$ and therapeutic strategies to

combat $A D$.

Figure 1.2 An overview of $A \beta$ pathogenesis hypothesis. 4

Figure 1.3 Alternative splicing of APP and the amyloidogenic and non-amyloidogenic pathways.

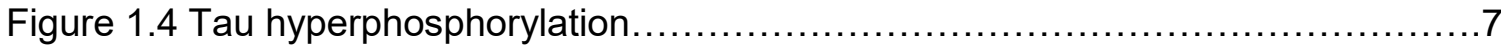

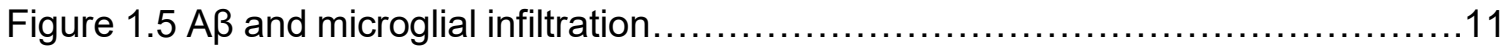

Figure 1.6 A $\beta$ clearance by microglia via triggering receptor expressed on myeloid Cells 2 ........

Figure 1.7: The Semipermeable Blood Brain Barrier and transmigration route of the Nanoparticles

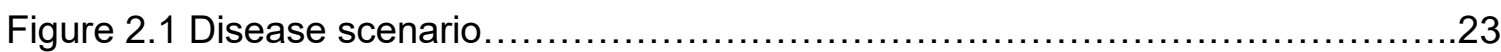

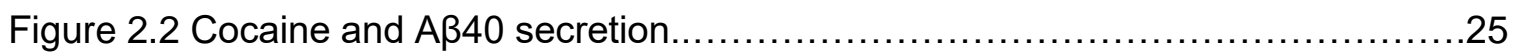

Figure 2.3 Effect of Withaferin $A(W A)$ on amyloid beta $(A \beta)$ secretion....................33

Figure 2.4 WA inhibits $A \beta 1-40$ in concentration dependent manner......................34

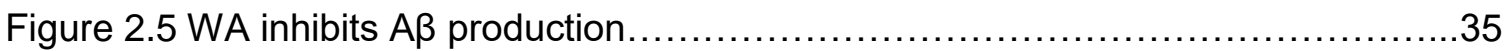

Figure 2.6 Tat induces increase in secreted $A \beta 40$ levels.................................

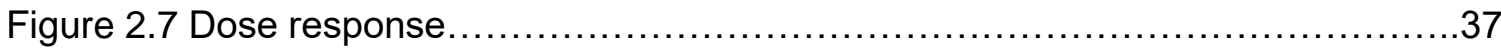

Figure 2.8 Coc induces increase in secreted $A \beta 40$ levels................................

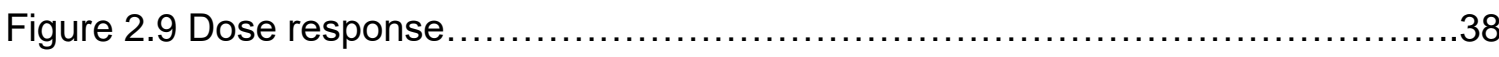

Figure 2.10 Effect of Tat and coc combination............................................

Figure 2.11 WA inhibits HIV-1 Tat induced A $\beta$-production, respectively.................41

Figure 2.12. WA reverses coc induced dendritic beading and cytoplasmic vacuoles.....42 
Figure 2.13 Quantification studies.

Figure 2.14 Congo red staining for Amyloid shows decreased amyloid in WA treated cells $(20 \mathrm{X})$

Figure 2.15 Congo red staining for Amyloid shows decreased amyloid in WA treated cells (10X)

Figure 2.16 WA effect on the gamma secretases.....................................46

Figure 2.17 WA effect on the Beta secretases.........................................4

Figure 3.1 Hypothesis of WA and CRID3 against inflammation .......................56

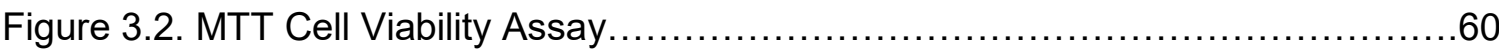

Figure 3.3 CRID3 inhibits Caspase- 1 and IL-1 $\beta$ protein expression.......................65

Figure 3.4 Designed CRID3 Liposomal Nanoformulation (CRID3-LNF) and its

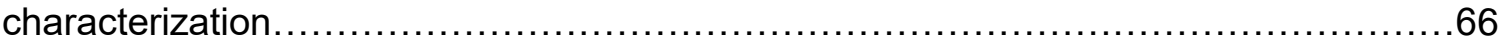

Figure 3.5 In-vitro effect of NF on the integrity of 3D BBB model.......................67

Figure 3.6 In-vitro NF transmigration across the 3D BBB model.........................68

Figure 3.7 The interdependent relationship between between $A \beta$, Microglial infiltration, production of inflammatory mediators and finally inflammation..........................69

Figure 4.1 The flow-plan of the strategy to increase the bioavailability of the drugs in to the CNS across the BBB

Figure 4.2 Preparation of Liposome Nano formulation ................................... 84

Figure 4.3 Developed in-vitro 3D BBB model demonstration............................87

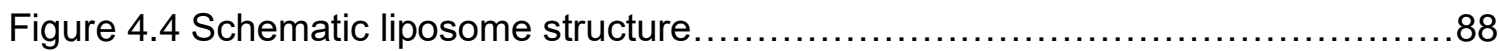

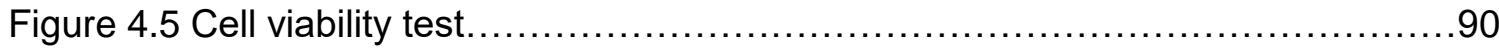

Figure 4.6 In-vitro WA Liposome transmigration and effect on BBB integrity compared

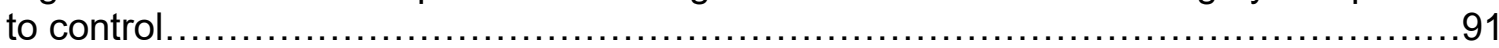

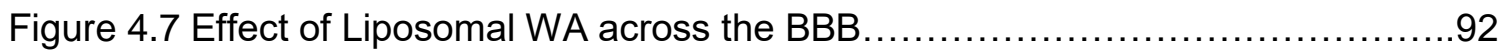

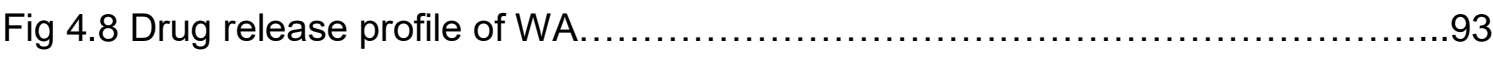


Figure 5.1 The stages of amyloid fibril formation and the associated cellular toxicity...100

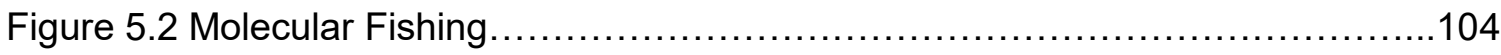

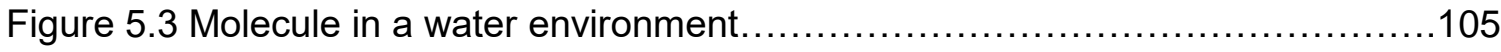

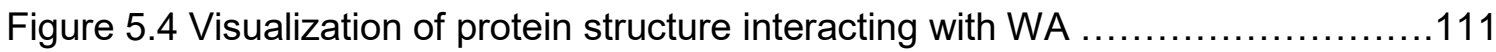

Figure 5.5 Visualization of the amino acid labelled protein drug complex..............111

Figure 5.6 Mesh view of the ab42 protein (golden ribbon) and its interaction with

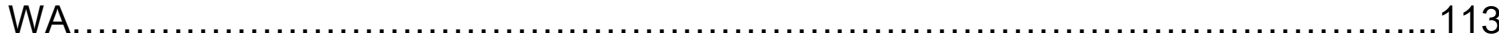

Figure 5.7 Surface view of the ab42 protein (golden ribbon) and its interaction with

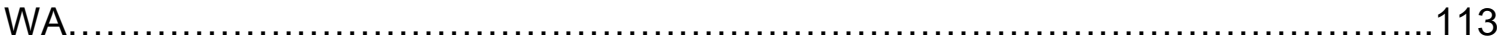

Figure 5.8 Demonstration of Hydrogen bonding between $A \beta 42$ and WA.............115

Figure 5.9 Two proposed mechanisms of WA's interaction with $A \beta 42$ fibrils............116

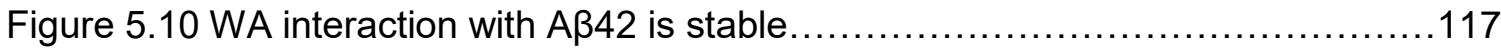

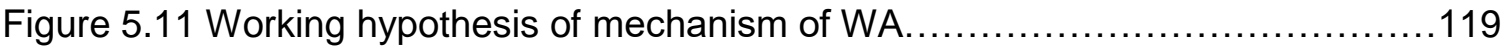




\section{ABBREVIATIONS AND ACRONYMS}

Alzheimer's disease

AD

APP intracellular domain

AICD

Anterior Pharynx-Defective-1

Aph-1

Amyloid Precursor like Proteins (1 and 2)

APLP1 and APLP2

Amyloid Precursor Protein

APP

Amyloid Precursor Protein-Like

APPL

APPs-alpha

APPsa

Amyloid Beta

$A \beta$

Beta-Secretase 1

BACE-1

Blood Brain Barrier

BBB

Cyclin-Dependent Kinase 5

CDK5

Central Nervous System

CNS

Cerebrospinal Fluid

CSF

Glycogen Synthase kinase 3

GSK3 $\beta$

HIV-1-associated neurocognitive disorders

HAND

c-Jun $\mathrm{NH}(2)$-Terminal Kinases

JNKs

Mitogen-Activated Protein Kinase

MAPK

Microtubule Affinity-Regulating Kinase

MARK

MAP Kinase

MEK

Neurofibrillary Fibrillary Tangles

NFTs

Protein Conformational Disease

PCD

Presenilin Enhancer-2

Pen2

Helically Wound Protein Filaments

PHF 
Protein kinase $\mathrm{A}$

PKA

Protein kinase C

PKC

Presenelin 1

PS1

Saxagliptin

SAX

Triggering Receptor Expressed On Myeloid Cells 2

TREM2

Trichostatin A

TSA

Valproic acid

VPA

Withaferin A

WA

Cocaine

Coc

Ashwagandha

$\mathrm{ASH}$

Nuclear factor kappa-light-chain-enhancer of activated B cells

NF-KB

Reactive oxygen species

ROS

Nitric oxide

NO

Cytokine Release Inhibitory Drug

CRID

Nod-like receptor protein

NLRP

CRID3 Liposomal Nanoformulation

CRID3-LNF

Withaferin A Liposomal Nanoformulation

WA-LNF

Tight junctions

TJ 


\section{CHAPTER 1: ALZHEIMER 'S DISEASE: PATHOGENESIS, DIAGNOSTICS AND THERAPEUTICS}

Parts of this chapter will appear in the International Journal of Nanomedicine, 2019 (Acknowledgements to Dove Medical Press as the original publisher of the article)

\subsection{Introduction}

Alzheimer's Disease $(A D)$ is a neurodegenerative and prominent protein conformational disorder (PCD) [1, 2] primarily caused by the aberrant processing and polymerization of normally soluble proteins [ $\underline{3}$ ]. The process of protein folding in the cell is tightly regulated, which encompasses various proteins, proteases, molecular chaperones that play a vital role in folding and clearance of misfolded protein along with regulation. However, genetic or sporadic environmental factors can alter this proof reading mechanism compromising the efficiency of protein folding process and resulting in non-native misfolded, dysregulated, destabilized, and aggregated proteins. This leads to PCD, in which the altered protein conformations contribute to cell toxicity, functional insufficiency and negative regulation. Protein misfolding is responsible for several neurological and metabolic disorders [2]. Proteins need to be folded into their final active state, corresponding to a particular conformation and have to be stable in that state for their proper functioning. A misfolded protein can undergo self- aggregation. Soluble neuronal proteins when misfolded attain altered conformations due to genetic mutation, external factors or ageing, and aggregate leading to abnormal neuronal functions and loss [4]. AD's discovery as a neurodegenerative disease is attributed to Alois Alzheimer, a German neurologist, who made first discovery when he examined a 51-year-old woman named Auguste Deter who was suffering with loss of memory, language issues, disorientation and hallucinations. Her autopsy revealed plaques and tangles in the cerebral cortex [5], 
which convinced him that this was unusual than typical dementia. His discovery was followed by further research which disclosed the presence of neuritic Amyloid Beta (A $\beta$ ) plaques in dementia patients [] $]$. Young onset of the disease is attributed to predisposition to Presenelin 1 (PS1) genetic mutation, which is rare but a potent cause [7]. Other neurodegenerative diseases associated with abnormal protein conformations, are Parkinson's disease (PD), Creutzfeldt- Jacob disease (CJD), Huntington's disease (HD), Machado-Joseph disease (MJD), which are caused due to abnormalities in a-synuclein, normal and pathological isoforms of prion protein $\left(\mathrm{PrP}^{\mathrm{c}}\right.$ and $\left.\mathrm{PrP}^{\mathrm{Sc}}\right)$, Huntingtin and Ataxin 3 proteins respectively. Here we have focused on understanding factors associated with AD pathogenesis, mechanisms, state of ART diagnostics and therapeutics available currently (Figure 1.1).

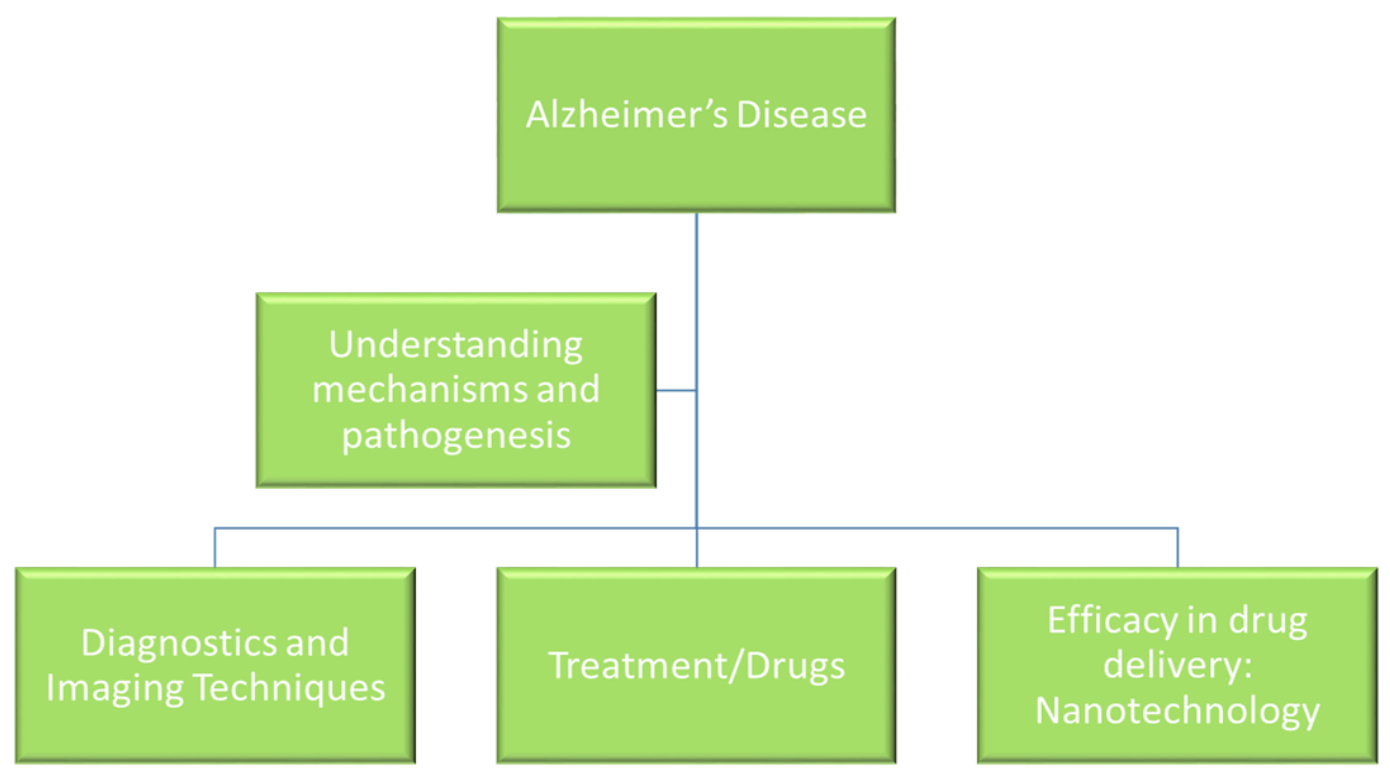

Figure 1.1 An overview of the fields of research that need to be elucidated to understand the pathophysiology of $A D$ and therapeutic strategies to combat AD. 


\subsection{AD Pathogenesis}

$A D$ is a highly complex and progressive neurodegenerative disease [8]. It is one of the leading cause of dementia cases globally. In US alone, approximately 5.3 million Americans have AD, out of which 5.1 million are 65 years or older and 200,000 have younger onset of $A D$ [9]. Commonly studied histopathological characteristics of $A D$ are extracellular aggregates of $A \beta$ plaques and intracellular aggregations of neurofibrillary tangles (NFTs), made of hyperphosphorylated microtubule-associated Tau protein. A $\beta$ plaques develop initially in the basal, temporal and orbitofrontal neocortex regions of brain and in later stages progress throughout the neocortex, hippocampus, amygdala, diencephalon and the basal ganglia. In critical cases, $A \beta$ is found throughout the mesencephalon, lower brainstem and cerebellar cortex. This concentration of $A \beta$ triggers Tau tangles formation, in the locus coeruleus, transentorhinal and entorhinal areas of brain. In critical stage, it spreads to the hippocampus and neocortex [10]. A $\beta$ and NFTs are the major players in the AD progression.

\subsection{Key events in the pathogenesis of $A D$}

The amyloid pathogenesis starts with the altered cleavage of Amyloid Precursor Protein (APP), an integral protein on the plasma membrane, by $\beta$-secretases (BACE-1) and $\gamma$ secretases to produce non-soluble $A \beta$ fibrils. $A \beta$ then oligomerizes and diffuses into synaptic clefts and interferes with the synaptic signaling [11, 12]. Consequently, it polymerizes into insoluble amyloid fibrils that aggregate into plaques. This polymerization leads to activation of kinases, which lead to hyperphosphorylation of the microtubuleassociated protein, tau, and its polymerization into insoluble NFTs. The aggregation of plaques and tangles is followed by microglia recruitment near plaques. This promotes microglial activation and local inflammatory response and contributes to neurotoxicity. 


\subsubsection{Structure and function of amyloid precursor protein}

APP belongs to a family of associated proteins including mammalian amyloid precursor like proteins (APLP1 and APLP2) and amyloid precursor protein-like (APPL) in Drosophila. It is an integral transmembrane protein with extracellular domains (Figure 1.2). APP, in diseased state, generates amyloidogenic fragments, by differential cleavage by enzymes [7]. The physiological functions of APP are still less understood, studies with transiently transfected cell lines show that APP moderates cell survival, growth and motility, along neutraneutrnnwith neurite outgrowth and functions, which are attributed to release of soluble ectodomains upon normal cleavage of APP $[13,14]$. The importance of APP has been highlighted by studies where neuronal abnormalities have been reported in animals injected with APP RNA interference (APP-RNAi) [15] and APP ectodomain intracerebral injections show improved cognitive function and synaptic density [16]. APP encodes type 1 transmembrane glycoprotein which is cleaved either via non-amyloidogenic pathway (normal state) or via amyloidogenic pathway (diseased state) [17]. APP releases various polypeptides which arise possibly due to alternative splicing, glycosylation, phosphorylation or complex proteolysis $[\underline{18}, \underline{19}]$.

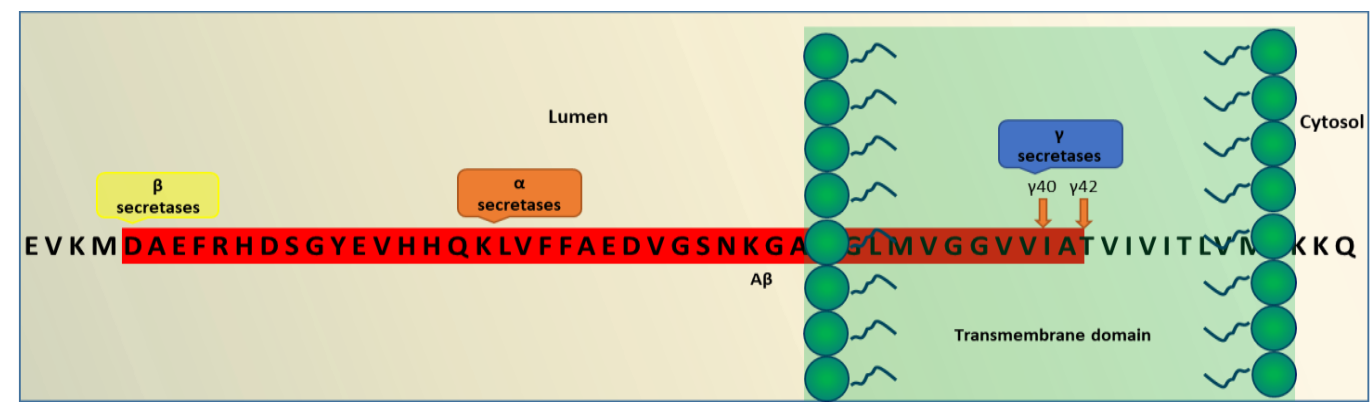

Figure 1.2 An overview of $A \beta$ pathogenesis hypothesis

This figure demonstrates the amino acid sequence of the $A \beta$ fragment and the location of action of $\alpha, \beta$ and $\gamma$ secretases in diseased neurons during amyloidogenic diseased pathway. 
APP comprises of 770 amino acids, out of which $A \beta$ includes 28 residues and additional 14 residues from the transmembrane domain of APP. At cleavage site, $\alpha$-secretase cleaves and secretes large soluble ectodomain APPs-alpha (APPsa) into the medium and C-terminal fragment (C83) is retained in the membrane, cleaved by $\gamma$ - secretase at residue 711 releasing soluble p3 peptide. Alternatively, in diseased state abnormal cleavage is done by $\beta$-secretase releasing truncated APPs $\beta$ and C-terminal fragment (C99) is retained in the membrane which is further cleaved by $\gamma$-secretase releasing insoluble $A \beta$ peptides. Cleavage of both C83 and C99 by $\gamma$-secretase releases the APP intracellular domain (AICD) into the cytoplasm which is soluble and translocates to nucleus for further gene expression function [5].

\subsubsection{Non-amyloidogenic pathway}

APP undergoes constitutive and regulated cleavage. Enzyme $\alpha$-secretase, cleaves APP at residue16-17 of $A \beta$ domain and yield soluble and nonpathogenic precursors. In neurons, ADAM10 and ADAM 17 (metalloproteases) are the major a-secretases. Processing by $\alpha$-secretase and $\gamma$-secretase generate, a small hydrophobic fragment $p 3$, which is soluble and has role in normal synaptic signaling, but its exact functions are not well elucidated. Cell-surface APP may get endocytosed resulting in endosomal production of $A \beta$, which leads to extracellular release, and aggregation of $A \beta$. The $\alpha$-secretase processing releases large soluble ectodomain APPsa that acts a neuroprotective factor and has a role in cell substrate adhesion. The presence of APPsa associates with normal synaptic signaling and adequate synaptic plasticity, learning and memory, emotional behavior and neuronal survival. Further, sequential processing releases AICD which translocates into nucleus and facilitates nuclear signaling and gene expression and regulation pathways [20]. 


\subsubsection{Amyloidogenic pathway}

APP is differently cleaved in case of the diseased state. A $\beta$ is released from APP through sequential cleavages by $\beta$-secretase, a membrane-spanning aspartyl protease with its active site situated in lumen and $\gamma$-secretase, an intramembrane aspartyl protease which is made up for four proteins; presenilin, nicastrin, Aph-1 and Pen-2 complexed together [21]. This complex attributes to the activity of $\gamma$ secretase, which produces insoluble and neurotoxic $A \beta$ fragments. $\beta$ secretase cleavage is first and rate-limiting step, making a cut at $\mathrm{N}$ terminus of $A \beta$. It removes the majority of the extracellular portion of the protein, leaving the C-terminal of APP [22], which is cleaved at C- terminus of A $\beta$ resulting in formation of $A \beta$ oligomers, which further polymerizes forming the aggregated plaques (Figure 1.3).

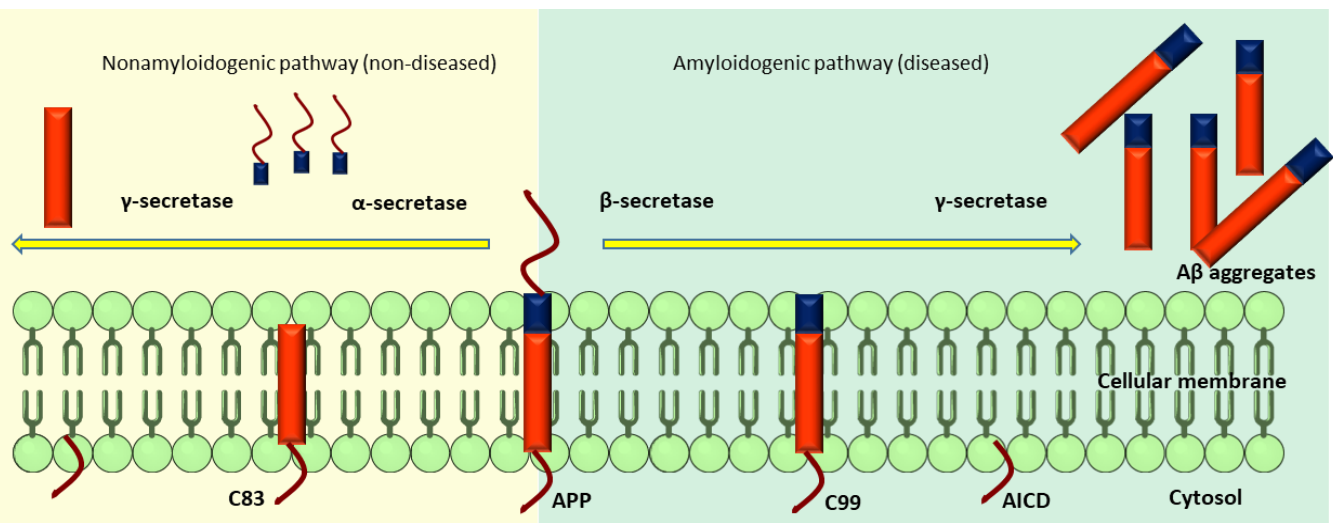

Figure 1.3 Alternative splicing of APP and the amyloidogenic and nonamyloidogenic pathways.

Cleavage by $\alpha$ and $\gamma$ secretase in normal state and alternative cleavage by $\beta$ and $\gamma$ secretase in diseased state.

There are two main types of $A \beta$ polymers, which contribute in plaque formation and induce neurotoxicity; $A \beta 40$ and $A \beta 42$ isoforms. $A \beta 40$ is abundant and neurotoxic when compared to $A \beta 42$, which is less abundant, insoluble and severely neurotoxic, more aggregation- 
prone and acts as toxic building fraction of $A \beta$ assembly. $A \beta 40$ is also neurotoxic and easily detectable in in-vitro studies. A $340 / 42$ aggregation results in blocked ion channels, altered calcium homeostasis, increased mitochondrial oxidative stress, diminished energy metabolism and glucose regulation, which contributes to deterioration of neuronal health and eventually cell death.

\subsection{Hyperphosphorylation of Tau and AD}

In addition to $A \beta$, another central pathological hallmark of $A D$ are the NFTs. These tangles are the result of hyperphosphorylation of the microtubule-associated protein Tau and its intracellular aggregates. NFTs are fragments of paired and helically wound protein filaments (PHF) in the cell cytoplasm of neurons and in their processes. Tau protein is a member of the microtubule-associated proteins (MAP) family, important for microtubular assembly and equilibrium in neuronal cells. Tau has a microtubule-binding domain and it co-assembles with tubulin to form mature and stable microtubule [23, 24]. It has the

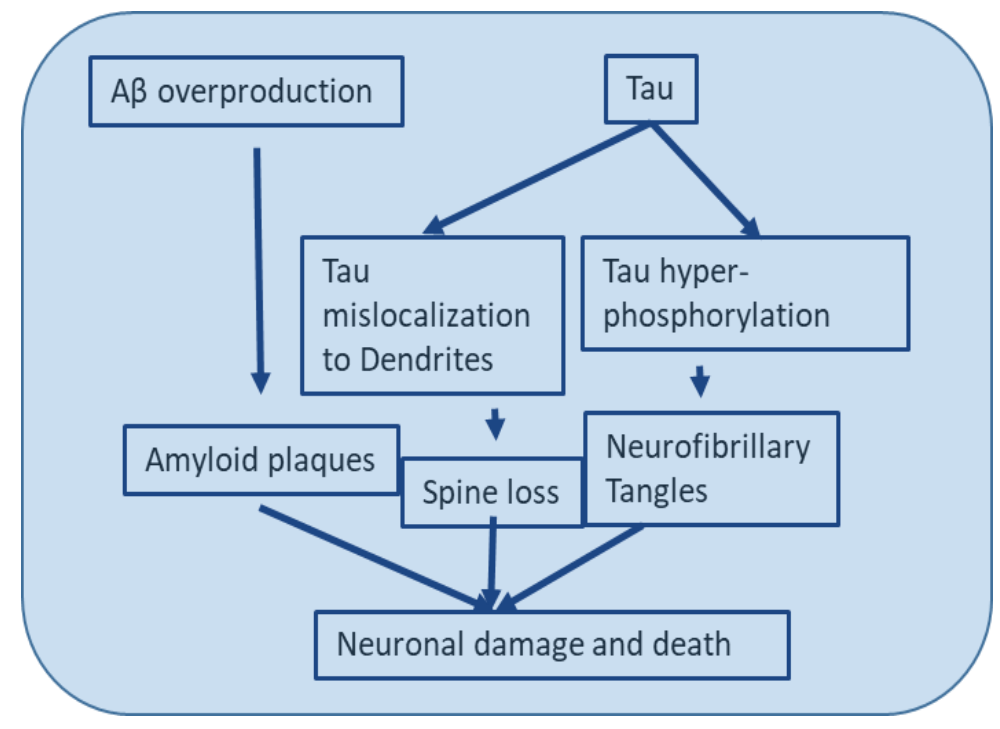

Figure 1.4 Tau hyperphosphorylation

Tau phosphorylation leads to instability of the microtubule and finally microtubule subunits fall apart leading to formation of insoluble and big Neurofibrillary Tangles. 
capability of forming interconnecting cross bridges between contiguous microtubules to form a proper stable network of microtubules and to hold them together. When the Tau protein encounters the kinases released due to abundance of $A \beta$ in the environment, it gets hyperphosphorylated. Its hyperphosphorylation leads to its dissociation from microtubules, the tubule gets unstable due to dissociation of tubule subunits and fall apart. These destabilized structures then convert into big chunks of tau filaments, which further aggregate into NFTs. These NFTs are straight, fibrillar and highly insoluble patches in the neuronal cytoplasm and processes, leading to abnormal loss of communication between neurons and signal processing and finally apoptosis in neurons [25] (Figure 1.4). Extracellular Tau aggregates from neurons spread the neurodegeneration in prion-like mechanism [26]. It has been reported that soluble $A \beta$ controls cleavage and phosphorylation of Tau for NFT generation [7].

Further, phosphorylation of tau is regulated by several kinases, including glycogen synthase kinase 3 (GSK3 $\beta$ ) and cyclin-dependent kinase 5 (CDK5), activated by extracellular A . Even though GSK-3 and CDK5 are primarily responsible kinases for Tau hyperphosphorylation, other kinases like Protein kinase $C(P K C)$, Protein kinase A (PKA), Erk2 a serine/threonine kinase, caspase 3, and caspase 9 have prominent roles too, which may be activated by $A \beta[27]$.

\subsection{GSK3B and CDK5 in AD}

GSK-3 regulates the cleavage of APP carboxy-terminal fragments. It has been showed that lithium and kenpaullone (GSK-3 inhibitors), prevent GSK-3 expression, and contribute to the inhibition of $A \beta$ production [28]. Hence, GSK-3 inhibitors might indirectly interfere with the generation of both $A \beta$ plaques and Tau tangles in AD. GSK3 $\beta$ activity in the 
mitochondria has been associated with increased oxidative stress [29]. Abnormal processing of APP leads to secretion of $A \beta$, which affects GSK-3 kinases leading phosphorylation of Tau protein. This leads to aggregation of Tau filaments which are insoluble and finally formation of huge masses of NFTs in the neurons [30].Thus, GSK3 $\beta$ plays a significant role in AD pathogenesis attributing to $A \beta$ production and $A \beta$-mediated neuronal death by increasing tau hyperphosphorylation. Additionally, A $\beta$ and CDK5 interactions affect Tau phosphorylation. This interaction leads to cleavage of adjacent proteins releasing cleaved peptides with lower solubility and longer half-lives; they may also phosphorylate distant proteins. Several studies focusing on identifying and classifying kinases accountable for pathogenic Tau hyperphosphorylation point towards primary pathogenic kinases GSK3 $\beta$ and CDK5, in addition to mitogen-activated protein kinase (MAPK), Erk 1 and 2, MAP kinase (MEK), microtubule affinity-regulating kinase (MARK), c-Jun NH(2)-terminal kinases (JNKs), p38, PKA, etc. [31, 32].

\subsection{Genetic mutations: Presenelin-1 mutation and AD}

APP is not the only gene associated with $A D$, presenilin gene (PS1 and PS2) which are part of $\gamma$ secretases may also undergo mutation [33]. Moreover AD patients may be predisposed to PS1 mutation leading to familial AD at young age [34]. The $y$-secretase complex encompasses four proteins: PS1, presenilin enhancer-2 (Pen-2), anterior pharynx-defective (Aph-1), and nicastrin. PS, an aspartyl protease, attributes to catalytic core of the complex. Pen-2 facilitates the maturation of PS, whereas Aph-1 stabilizes the complex [35]. Nicastrin acts as a receptor for $y$-secretase substrates. There are 179 PSEN1 and 14 PSEN2 gene mutations that participate in early-onset of autosomal dominant $A D$. These mutations favor production of more toxic form of amyloid; $A \beta 42$ compared to $A \beta 40$ which contributes in disease progression [36]. 


\subsection{Epigenetics and $A D$}

Epigenetics is the field which focuses on the interactions between genes, expression of the genotypes and various molecular pathways which modify genotype expression into respective phenotype [37]. Epigenetics exploring the neurological diseases, called as neuroepigenetics has developed fairly well and has been widely studied in the CNS associated diseases comprising of learning, motor, behavior and cognition pathologies and disorders $[\underline{38}, \underline{39]}$. Epigenetics is important to understand the depth of effect of environment or paternal genes, nutritional habits, trauma, stress or learning disabilities, exposure to chemicals or drug addiction, on the DNA and resulted structural disturbances, mutations or changes $[\underline{40}, \underline{41}]$. The involvement of epigenetics has recently been explored in case of $A D$ [42]. The onset of $A D$ and its progress involves complex interplay of various factors like ageing, genetic mutations, metabolic and nutritional disorders, exposure to the environment and involvement of social factors [43]. There are fair chances that additional factors in addition to ageing, for examples, hypertension, disorders like diabetes and obesity, and inflammatory disorders may be inducing epigenetic changes and may induce AD-like pathogenesis in young age. DNA methylation patterns in brain and aging is possible [44]. From the studies involving various regions of the brain, an association between DNA methylation and ageing was reported [45]. Since DNA epigenetic mechanisms have a role in memory formation and its maintenance, like decrease in DNA methylation deteriorates neuronal plasticity, leading to memory loss, it is speculated that understanding of epigenetic mechanism is important to understand ageing and associated complexities in AD patients [46]. In addition to DNA methylation, histone modifications may also play an important role. Studies have explored histone acetylation in APP/presenilin1 double mutant transgenic mice, where impairment in associative learning was connected to H4K14 histone acetylation reduction [47]. Additionally, HDAC inhibitors 
(HDACls) are believed to have effect on $A \beta$ production and aggregation in $A D$ mice. Therefore, the studies involving their inhibitors like trichostatin A (TSA), valproic acid (VPA), and vorinostat (SAHA) are important to target HDAC associated epigenetic mechanism involved with ageing, to target $A D[48]$.

\subsection{Microglial infiltration during plaque formation: leading to neurodegeneration}

There are extensive evidences that associate neuroinflammation with AD progression. In addition to extracellular $A \beta$ plaques and NFTs, microglial infiltration in response to these aggregates exacerbates $A D$ pathogenesis. The extracellular and intracellular $A \beta$ and tangles cause extreme toxicity, which results in synaptic damage, increased reactive oxidative stress, leading to increased microglial infiltration in vicinity to the plaques. Microglia are the resident phagocytes in the CNS, and play vital role as immune cells, in

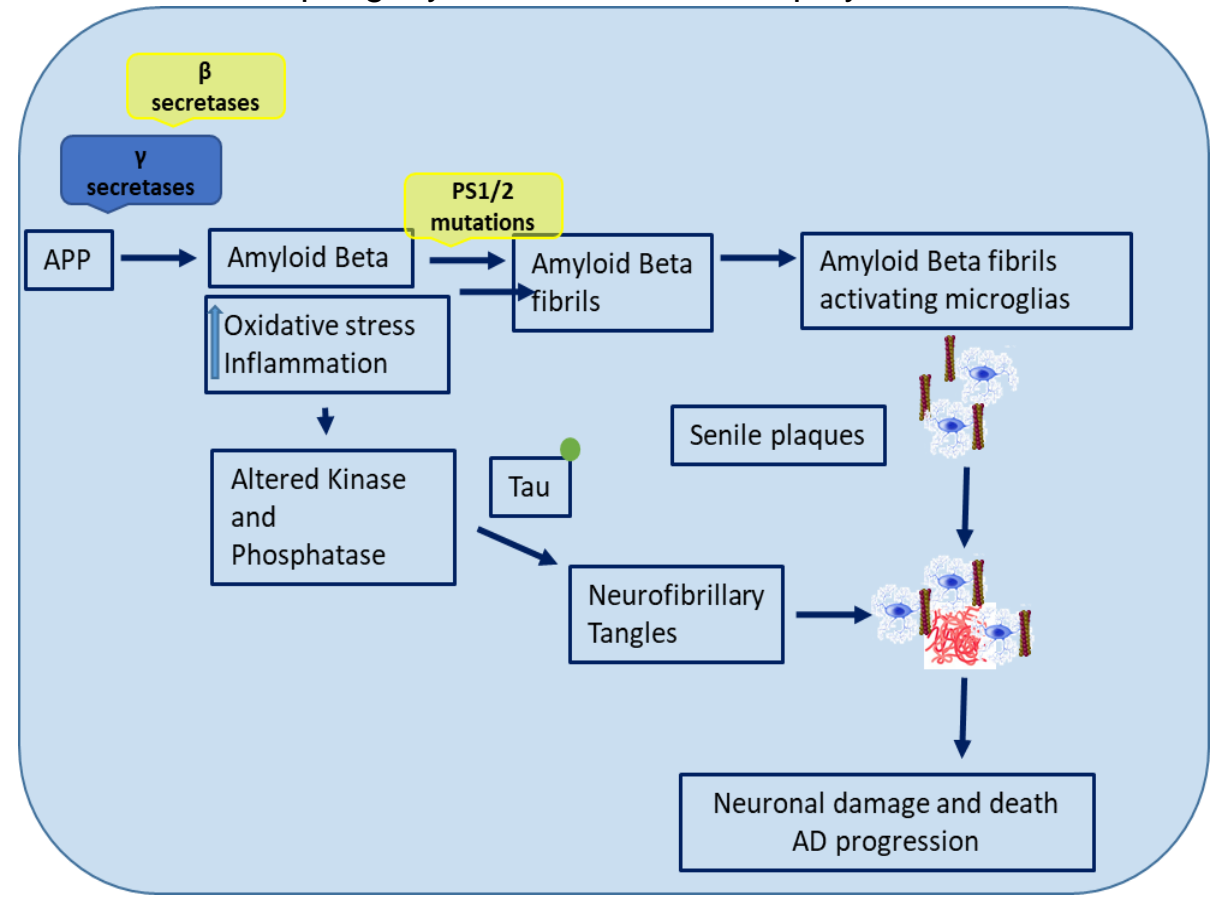

Figure 1.5: $A \beta$ and microglial infiltration

The extracellular and intracellular $A \beta$ and tangles cause extreme toxicity, which results synaptic damage, increased reactive oxidative stress that then leads to microglial infiltration around the plaque areas. 
the maintenance of neuronal plasticity and synapse remodeling [49]. Microglia get activated by protein accumulation which act as pathological trigger, and they migrate to the location and initiate an innate immune response [두 (Figure 1.5).

In neurotoxic or neurodegeneration scenario, abnormal amounts of aggregated $A \beta$ plaques activate Toll-like receptors on microglia leading to microglial activation leading to secretion of pro-inflammatory cytokines and chemokines [50]. In AD, microglia can bind to $A \beta$ via cell-surface receptors, including SCARA1 (scavenger receptor A-1), CD36, CD14, $\alpha 6 \beta 1$ integrin, CD47, RAGE (receptor for advanced glycation end products) and Toll-like receptors $[\underline{51}, \underline{52}]$. SCARA-1 is associated with $A \beta$ clearance of, whereas CD36 and RAGE contribute in microglial activation by $A \beta$ [53]. Upon receptor binding of $A \beta$, microglia endocytose $A \beta$ oligomers and NFTs fibrils, which are eliminated by endolysosomal degradation. Microglial proteases like neprilysin and insulin-degrading enzyme (IDE) play major roles in the degradation [54]. However, in severe cases of AD, microglial clearance of $A \beta$ is inefficient due heavy $A \beta$ load and increased localized cytokine concentrations which downregulate the expression of $A \beta$ phagocytosis receptors, leading to minimal $A \beta$ clearance [55]. One of the factors behind compromised $A D$ clearance by microglia is mutation in triggering receptor expressed on myeloid cells 2 (TREM2). TREM2 mutations are associated with increased AD severity. TREM2 is a cellsurface receptor of the Ig-superfamily highly expressed on microglia and involved in mediating phagocytic clearance of neuronal debris. It also binds anionic carbohydrates, bacterial products and phospholipids, and transmits intracellular signals through the associated transmembrane adaptor DAP12 (Figure 1.6) and further phosphorylation of downstream mediators [드]. 


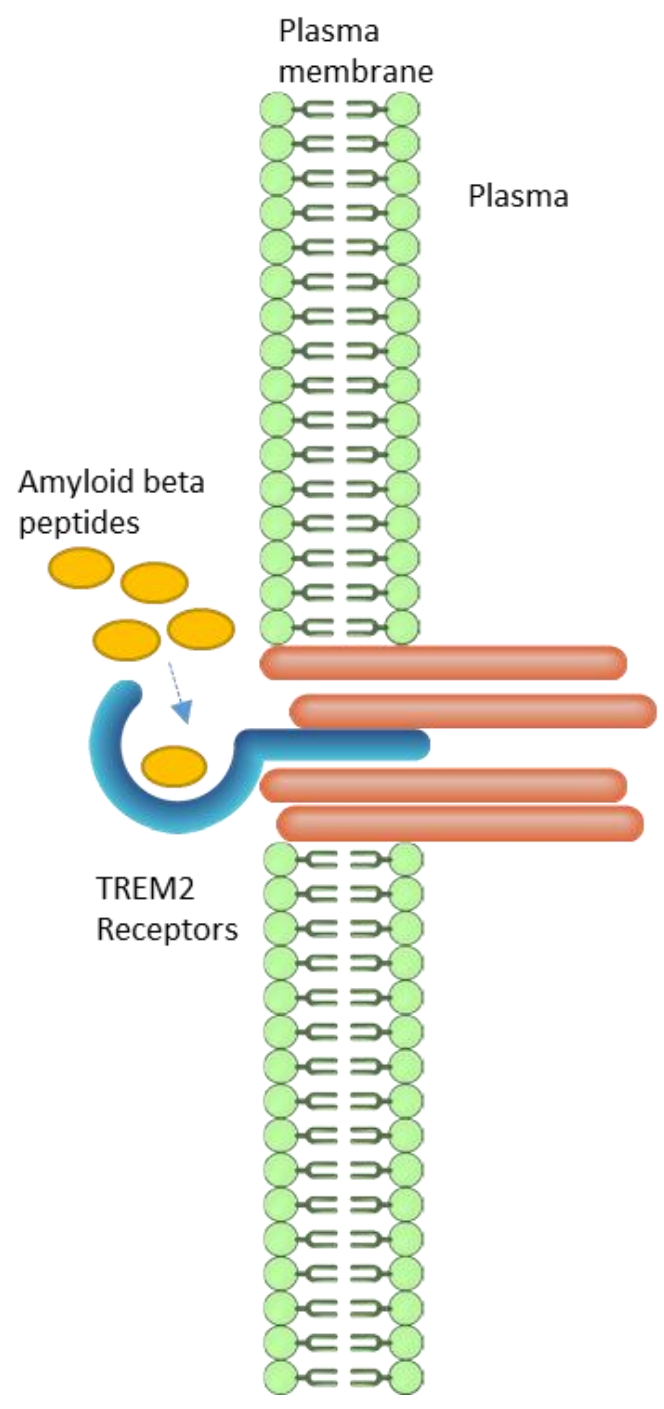

Figure 1.6 A $\beta$ clearance by microglia via triggering receptor expressed on myeloid cells 2 (TREM2)

During $A D$, a rare Arginine-47-Histidine $(\mathrm{R} 47 \mathrm{H})$ mutation of TREM2 is reported which plays a potent role in aggravating the risk [57]. This mutation leads to inability of the receptors to clear $A \beta$ from the CNS contributing to $A \beta$ accumulation and further intensification of pathogenesis in AD patients. TREM2 mutations in microglia contribute towards $A \beta$ induced toxicity. 


\subsubsection{Microglia and $A \beta$ clearance}

Microglia or resident macrophages of the CNS are derived from embryonic yolk sac and later enter into CNS [됴] . The role of microglia is important with respect to protection and maintenance against the neurodegenerative disorders and their pathogenesis in the CNS [59-62]. The primary role of microglia in a normal state is to infiltrate at the pathogenesis site and alters its receptor expression based on the pathogenesis factors, and perform both pro- or anti-inflammatory activity, including clearance of $A \beta$ [63]. Due to $A \beta$ chemotactic effect, microglia infiltrate and gather near the dense and insoluble amyloid plaques [64]. During excessive load of $A \beta$, microglia gets outnumbered and their clearance mechanism does not work efficiently, instead this leads to more microglial infiltration which then via pro-inflammatory cytokines (Interleukin-1 (IL-1), tumor necrosis factor (TNF- $\alpha$ )) and nitric oxide (NO) result in heightened levels of $A \beta$ aggregation [65]. IL-1 $\beta$ is also released due to $A \beta$ accumulation [66]. Furthermore, the classical microglial activation is associated with degradation and clearance of $A \beta$ peptides through phagocytosis [67], and clearance through G protein-coupled receptor (GPCR)-mediated signaling [ $\underline{68}, \underline{69}]$.

\section{$1.9 \mathrm{~A} \beta$ and HIV-1 associated neurological disorders}

Currently, disease associated neurological disorders are the biggest area of concern. In this era of Anti-Retroviral Therapy (ART), with the increase in the aged Human Immunodeficiency Virus (HIV) patients, the incidences of dementia or other neurocognitive functions are increasing in the aged patients when compared to younger patients. HIV-1associated dementia risk in these patients is three times higher than in younger people [70]. In $A D$, there are neurological dysfunctions due to abnormal accumulation of extracellular $A \beta$ produced by alternate cleavage of the APP. The A deposition is also reported to occur in the cortex of HIV patients when compared to age matched non-HIV 
controls [71-74]. The studies to explain the increased AD like indications, with increase in $A \beta$ levels, during the HIV infection are in embryonic stage. It is hypothesized that $A \beta$ deposition may be a common factor which aggravates in HIV-1 infection thus contributing towards HIV associated neurocognitive disorders (HAND) and causing subtle neurodegeneration especially in hippocampal neurons. Additionally, increased A $\beta$ in HIV1-infected brains may affect the brain vascular functions contributing to Blood Brain Barrier (BBB) dysfunction. If $A \beta$ is the common factor between $A D$ and HIV-1 disease scenarios, it becomes imperative to address the targeting of $A \beta$ pathway with a single and efficacious drug molecule.

\subsection{State of Art: AD Therapeutics}

In $A D$, there are constant studies going on towards targeting various stages of neurotoxicity by inhibiting the production and aggregation of the misfolding proteins $A \beta$ and Tau, their spread and induced toxicity [75]. The majority of AD therapeutic approaches are focused on reducing levels of toxic forms of $A \beta$ and Tau, the broad scope of neurodegenerative processes underlying both early- and late-stage AD. Several drugs have been analyzed and reached Phase 1, 2 and 3 of clinical trial levels. A detailed table summarizes the drugs specific to amyloid, which are studied and target sufficiently fundamental and proximate degenerative mechanisms [ $[\underline{76}, \underline{77]}$ (Table 1.1).

However, all these current therapeutics (e.g. rivastigmine, galantamine and donepezil) target dementia associated secondary features and do not directly involve against specific AD characteristics. Therapy failure frequently occurs due to the unfavorable pharmacokinetics and pharmacodynamics of drugs. Pharmacotherapy failure is the result of inadequate physical chemistry of drugs (such as hydrophobicity), unfavorable 
absorption by biological membranes, unfavorable pharmacokinetic parameters (such as intense and plasma metabolism), instability of drugs (oxidation, hydrolysis, or photolysis), and toxicity to tissues (hepatotoxicity, neurotoxicity, or kidney toxicity).

\section{Table 1.1: Drugs specific to amyloid that are studied and target sufficiently against fundamental and proximate degenerative mechanisms}

\begin{tabular}{|c|c|c|c|}
\hline Agents & Drug Trials & Target & Action \\
\hline Aducanumab & Phase 1 & Antiamyloid & Monoclonal antibody \\
\hline Albumin + immunoglobulin & Phase 1 & Antiamyloid & Polyclonal antibody \\
\hline AZD3293 (LY3314814) & Phase 1 & Antiamyloid & BACE1 inhibitor \\
\hline CAD106 & Phase 1 & Antiamyloid & Amyloid vaccine \\
\hline CNP520 & Phase 1 & Antiamyloid & BACE inhibitor \\
\hline E2609 & Phase 1 & Antiamyloid & BACE inhibitor \\
\hline Gantenerumab & Phase 1 & Antiamyloid & Monoclonal antibody \\
\hline Nilvadipine & Phase 1 & Antiamyloid & Calcium channel blocker \\
\hline Solanezumab & Phase 1 & Antiamyloid & Monoclonal antibody \\
\hline ATP & Phase 2 & Antiamyloid & amyloid misfolding and toxicity \\
\hline Atomoxetine & Phase 2 & Antiamyloid & Adrenergic uptake inhibitor \\
\hline AZD0530 (saracatinib) & Phase 2 & Antiamyloid & Kinase inhibitor \\
\hline Crenezumab & Phase 2 & Antiamyloid & Monoclonal antibody \\
\hline JNJ-54861911 & Phase 2 & Antiamyloid & BACE inhibitor \\
\hline Posiphen & Phase 2 & Antiamyloid & Selective inhibitor of APP production \\
\hline Sargramostim (GM-CSF) & Phase 2 & Antiamyloid & amyloid removal \\
\hline UB-311 & Phase 2 & Antiamyloid & Monoclonal antibody \\
\hline Valacyclovir & Phase 2 & Antiamyloid & Antiviral agent \\
\hline Aducanumab & Phase 3 & Antiamyloid & Monoclonal antibody \\
\hline KHK6640 & Phase 3 & Antiamyloid & Amyloid aggregation inhibitor \\
\hline Lu AF20513 & Phase 3 & Antiamyloid & Polyclonal antibody \\
\hline LY2599666 + solanezumab & Phase 3 & Antiamyloid & Monoclonal antibody combination \\
\hline NGP 555 & Phase 3 & Antiamyloid & Gamma-secretase modulator \\
\hline MK-8931 (verubecestat) & Phase 3 & Antiamyloid & BACE Inhibitor \\
\hline
\end{tabular}

Several therapeutic strategies have been proposed and attempted for the reduction in abnormal levels of $A \beta$. Further, several drugs were tested for targeting $A \beta$ degradation, but majority showed promising results in in-vivo studies, and unfortunately, were not able to clear the human clinical trials. This failure creates an urgent need to develop strategies, which are soluble, stable, target specific, to AD associated hallmarks, and potent to pass the human clinical trials. Many of the available drugs lose their efficacy while crossing the 
BBB and are minimally bioavailable in the brain. This requires a new area of study, which expands into efficacious neuroprotective strategies, specific to the brain. Nanoparticles are intriguing candidates for this purpose because of their potential for multifunctionalization, mimicking the physiological mechanisms of transport across the BBB, which is a semipermeable barrier protecting the brain from potential hazardous substances in the blood flow; however, it also prevents the passage of vital neurotherapeutics.

\subsection{Diagnostics for AD: Labeling and imaging}

The current AD diagnostics are primarily based on the neuropsychological testing. Clinical diagnosis of $A D$ requires neuroimaging methods and monitoring accepted biomarkers e.g. concentrations of $A \beta$ peptides ( $A \beta 1-42$ / $A \beta 1-40$ ratio) as well as of total (T-Tau) and hyperphosphorylated tau (P-tau) proteins in the cerebrospinal fluid (CSF). Amyloid oligomers and plaque accumulation can be imaged by 1 florbetapir F-18 (or alternative C11 Pittsburgh compound B, PiB ligand) Positron Emission Tomography (PET) but nonlinear association between $A \beta$ content in CSF and PET scan remains of concern. However, CSF sampling is relatively invasive and is not always well tolerated or feasible for elderly patients. Noninvasive imaging methods such as Fluoro-2-deoxy-Dglucose (FDG)-PET, which gives insights into the brain metabolism, are of great clinical utility. Certainly, altered cerebral metabolism (both hyper- and hypo-metabolism) has been associated with different stages of $A D$. Magnetic resonance imaging (MRI) at increased field strength and resolution is another helpful, non-invasive approach for identification of the functional abnormalities. MRI is utilized for detection and identification of amyloid plaques utilizing Iron oxide nanoparticles $\left(\mathrm{Fe}_{2} \mathrm{O}_{3}-\mathrm{NPs}\right)$ as contrast agents or tagged with fluorescent probes to make the detection efficient [78]. The $\mathrm{Fe}_{2} \mathrm{O}_{3}-\mathrm{NPs}$ are reported to 
bind to $\mathrm{N}$ terminal of $A \beta$, aiding in better imaging. Additionally, non-fluorescent or fluorescent rhodamine tagged $\mathrm{yFe}_{2} \mathrm{O}_{3}-\mathrm{NPs}$ have been reported to label $A \beta$ fibrils selectively and remove them, by employing external magnetic field $[\underline{79}, \underline{80}]$. In addition to $\mathrm{Fe}_{2} \mathrm{O}_{3}-\mathrm{NPs}$, there are reports of polystyrene-block-poly (n-butyl cyanoacrylate) (PS-bPnBCA) NPs encapsulating Thioflavin $T$, to be able to interact and target $A \beta[\underline{81}, \underline{82}]$. Gold nanoparticles for their use in MRI as contrasting agents to study structural stages of $A \beta$ self-assembly [83] and Fluorescent semiconductor nanocrystals (quantum dots) for labeling [84].

For sensing the soluble forms of $A \beta$ in the CSF, the ultrasensitive NPs-based bio-barcode system detecting soluble oligomers with the aid of oligonucleotide (DNA barcode)modified gold nanoparticles (AuNPs) and magnetic microparticles (MMPs), functionalized with monoclonal/polyclonal antibodies [85] are utilized. Additionally, electrochemical sensing utilizing click chemistry, which involves AuNPs and assembled monolayers on it, to interact with $A \beta$ peptide are promising [86]. Ultrasensitive electrical detection method for A $\beta 42$ by scanning tunneling microscopy (STM) are currently employed [87]. These recently introduced technological and conceptual achievements considerably provide improved $A D$ diagnosis strategies. Upon $A D$ diagnosis, the therapeutic and treatment strategies can be tactfully designed. The diagnostics and imaging techniques include, for example, nanoparticle based sensitive early phase detection of AD biomarkers in the CSF samples from the patients. Nanomaterials can also be used as contrast agents for imaging $A \beta$ aggregated plaques, this highlights a very important quality of the nanmaterials which upgrade their usage in the research experiments. It is imperative to understand the role of nanoparticles in increasing the efficacy and bioavailability of the drug across the BBB into the CNS. 


\subsection{Need of nanotechnology as Therapeutic strategy}

There are promising drugs against $A \beta$ toxicity [88], but these drugs fail to cross and are not available in pharmacologically significant concentrations in the CNS. Typically, pharmaceuticals consist of small molecules, which do not cross the BBB on their own, therefore, the nanocarriers to boost drug delivery are required. Availability of drugs in the CNS is the major issue faced in the field of therapeutics against AD. The main reason is the presence of a fully functional semi permeable BBB, which poses as an obstacle for transmigration of neurotherapeutic molecules (like drugs, peptides, vectors, molecules) across it, into the CNS.

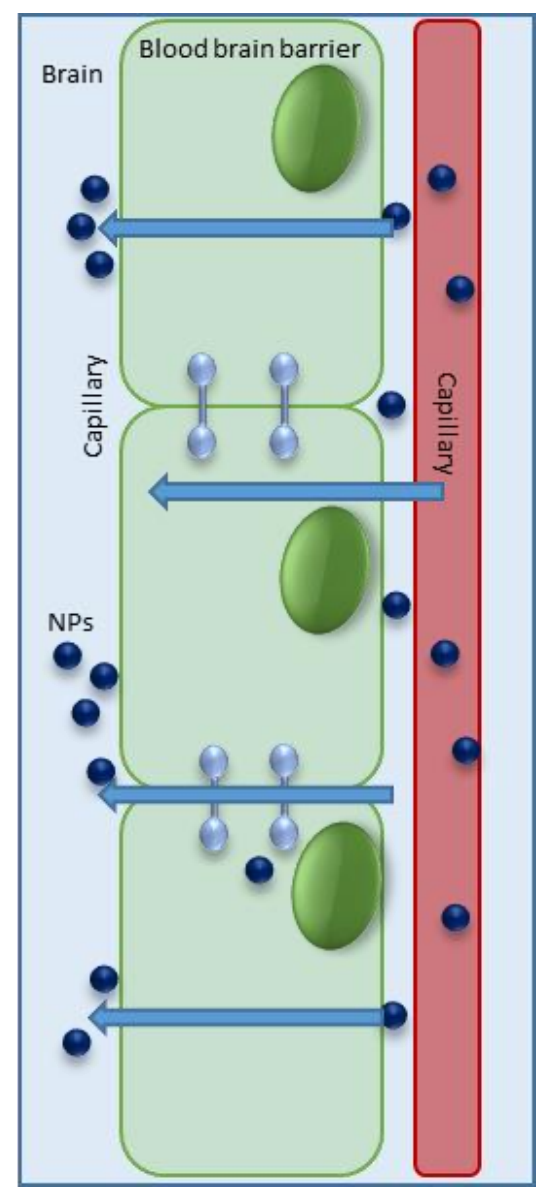

Figure 1.7 The Semipermeable Blood Brain Barrier and transmigration route of the Nanoparticles 
In addition, the BBB also negatively affects drug efficacy and tolerance, because large doses of drugs are needed to reach levels above the minimum effective concentration in the brain. Nanoparticulate systems offer an opportunity to overcome such problems and can be used as Trojan systems for transporting active molecules across the BBB (Figure $1.7)$, thus reducing toxicity and improving therapeutic efficacy [89, $\underline{90]}$.

The use of drugs in nano-platforms or nanodevices results in the enhancement of their pharmacokinetics and pharmacodynamics, as well as reduces the toxicity. It is advantageous in an essential aspect in nanomedicine for delivery and controlled release of drugs onto targeted disease sites. Thus, the effectiveness of a treatment can be increased by incorporating nanotechnology-based drug delivery systems. Some of these new platforms, which aim to improve the bioavailability, pharmacokinetics, and pharmacodynamics of drugs while reducing their side effects, are well studied. Recent nanotechnology advancements propose effective diagnostic and therapeutic options. Targeted drug delivery with the aid of nanoparticles (100nm in size) can effectively increase the drug bioavailability cross the BBB into the CNS with minimal or no side effects. Furthermore, these nanomaterials are designed to be biocompatible hence reducing the toxicity, and with the modifications in their magnetic and optical properties, they may be an efficient alternative agents for an early diagnosis [91]. For example, a study shows the delivery of Saxagliptin (SAX); a dipeptidyl peptidase-4 enzyme inhibitor molecule, which is explored for its activity in the therapy of $A D$, with the aid of the chitosanL-valine conjugate used to prepare nanoparticles encapsulating SAX. These nanoparticles were stable and crossed the BBB efficiently [92]. In Chapter 4 of thesis, we will have discussed about the importance of nanotechnology in effective drug delivery across the BBB and the importance of Liposomal Nanoformulations. 


\subsection{Conclusion and Future perspectives}

$A \beta$ was first sequenced from the meningeal blood vessels of $A D$ patients and individuals with Down's syndrome nearly 20 years ago [93-95]. A $\beta$ peptide was recognized as the primary component of the senile (neuritic) plaques of AD patient brain tissue [96]. These discoveries marked the beginning of research on AD. The cloning studies of APP gene and its localization to chromosome 21 [97-99] in addition to the fact that trisomy 21 leads to $A D$ neuropathology [100] highlighted that the $A \beta$ accumulation is the primary event in AD pathogenesis. Additionally, mutations in the APP gene which cause hereditary cerebral hemorrhage and amyloidosis also, may cause A $\beta$ deposition in the AD brain [101, 102] [103-106]. AD is one of the most common neurodegenerative diseases today, but unfortunately there is no cure available currently [107]. Several therapies are currently employed to combat with the cognitive and behavioral deficits associated with AD. Development of a targeted efficacious therapeutic approach against $A D$ is still in its developmental stage, thus the need of the hour is to look upon the cellular factors closely associated with disease pathogenesis and target them for improvement of the quality of life for AD patients. Cellular factors discussed in this project like A,$A P P$, and $\beta$ and $\gamma^{-}$ secretases, could be a key target for designing the therapeutic approach. It is utmost important to understand the limitations of drug bioavailability in the CNS due to the tightly controlled permeability of BBB. Nanomedicine offers an attractive approach for delivering drugs across the BBB [108-111]. Nanotechnology pertains to nano sizes of drug and their efficient delivery and controlled release in the brain by external magnetic field, which could be a promising factor in therapeutics for AD. Nannotechnology is also important for characterizing and visualization of the drug bound with nanomaterials, which makes it easier to track and confirm their targeting efficacies. Need of the hour is to unravel the mechanisms of the pathogenesis of $A D$, its early detection using state-of -the-art 
biosensing devises, specific targeting of the molecules associated with the disease manifestation, and efficient delivery of optimum drugs to the brain using novel nanotechnology approaches. Further, studies of comorbidities of AD with other diseases or viral infections are important for betterment of therapeutic approaches. 
CHAPTER 2: WITHAFERIN A SUPPRESSES BETA AMYLOID IN APP EXPRESSING CELLS: STUDIES FOR TAT AND COCAINE ASSOCIATED NEUROLOGICAL DYSFUNCTIONS

Parts of this chapter appear in the journal Frontiers in Aging Neuroscience. Published online 2018 Sep 27. doi: 10.3389/fnagi.2018.00291

\subsection{Introduction}

The overall life expectancy of people living with HIV (PLWH) has increased moderately due to introduction of effective anti-HIV therapies [112]. As per WHO Number of AIDS related death decreased from 1.5 million (2010) to 1.1 million (2015) globally [113]. Longer drug (anti-retroviral) consumption and virus living cycle leads to increased prevalence of HAND [114]. Additionally, PLWH ( 2 million as per World Health Organization, 2018) are more prone to the risk of developing neurological diseases like $A D$ and (AD)-like neurocognitive problems [115]. HIV-infection and associated neurological disease synergism has become a pressing health issue to be managed, globally' because HIV-

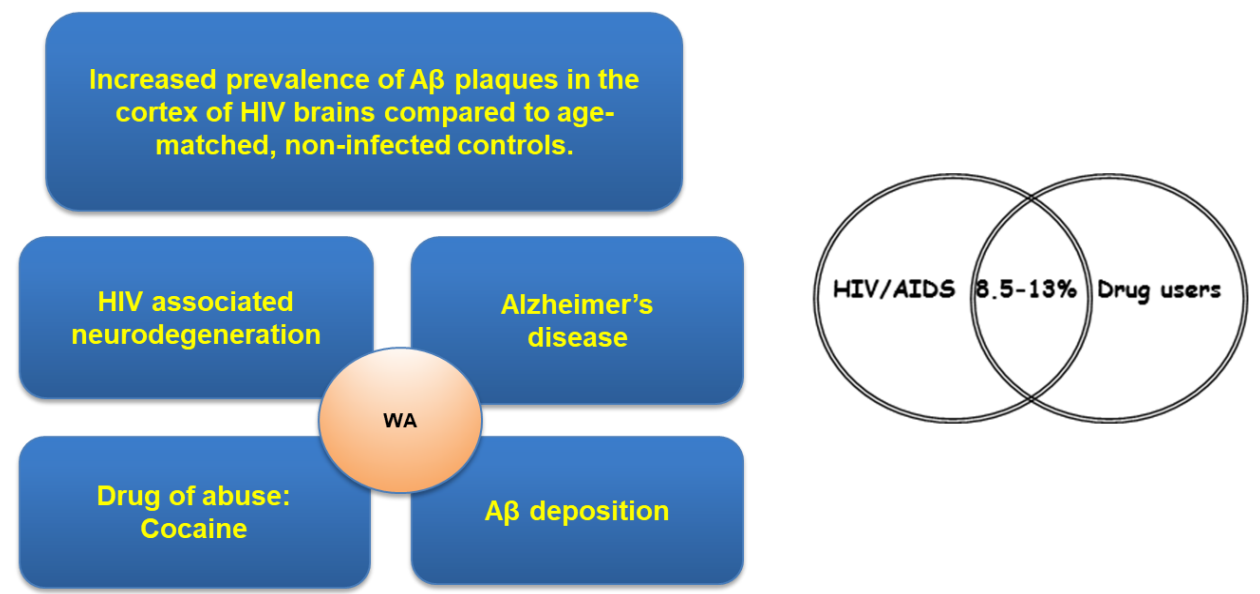

Figure 2.1 Disease scenario

$A \beta$ is a common factor between HIV associated neurocognitive dysfunctions and AD. Drug of abuse also contribute towards disease pathogenesis. 
infection progression facilitates $A D$ like pathology [116, 117] (Figure 2.1). However, neurological disorders are irreversible but investigating novel therapies of better efficacy to manage these serious disorders without side effects are urgently required.

As discussed in Chapter 1, AD is a prominent neurodegenerative disease, characterized as a progressive impairment of memory and neurocognitive functions due to abnormal accumulation of extracellular $A \beta$ and intracellular neurofibrillary tangles (NFTs) [118-121]. Alternative or abnormal cleavage of integral membrane APP by $\beta$ and $\gamma$ secretases $[\underline{122}$, $123]$ lead to abnormal $A \beta$ processing, resulting into insoluble $A \beta$ aggregation $[124,125]$ into extracellular insoluble senile plaques $[\underline{123}, \underline{126}, \underline{127}]$. This $A \beta$ accumulation leads to decreased neuronal health and stability, increased deterioration, synaptic depression [128-130], oxidative stress [131-133], augmented neuronal dysfunctions and inflammation $[134, \underline{135}] \underline{136}]$.

Studies support that impairments caused by $A \beta$ aggregation, become worst with HIV-1 infection [137, 138] and drugs of abuse. HIV patients have augmented $A \beta$ plaques deposition in the brain compared to HIV negative individuals [70-72]. Moreover, $A \beta$ aggregations are studied to be increased in cortex of HIV brains when compared to age matched non-HIV controls $[\underline{139}, \underline{140}]$. In other words, $A \beta$ may be considered as a common factor between HIV and $A D$ associated neurological dysfunctions. HIV induced $A \beta$ neurotoxicity could be due to either the entire HIV, or mainly due to the presence of neurotoxic Tat (transactivator of transcription) protein [141]. Even though ART targets all the active HIV, Tat could still be produced by the provirus in the viral reservoirs, such as the brain [142]. Tat protein as a neurotoxin, plays a prominent role in HIV neuropathogenesis as it gets secreted extracellularly and has the ability to cause 
neurotoxicity in the healthy cells $[143, \underline{144]}$. Tat may have specific reaction with the $A \beta$ in the CNS and facilitate $A \beta$ aggregation, in the CNS [145].

Neurotoxic HIV-1 Tat protein may be affecting amyloidogenesis through various indirect mechanisms and can have direct interactions with $A \beta$ fibers and plaques. Tat is capable of increasing $A \beta$ aggregation and may provide increased rigidity and mechanical resistance to the fibrils [145]. Whereas the indirect action of Tat activity can be supported by its interaction with human neprilysin, which is reported to function in $A \beta$ degradation, including both monomeric and pathological oligomeric forms of $A \beta$ [146]. HIV-Tat inhibits the activity of Neprilysin from degrading the amyloid oligomers into inert fragments [142].

Another factor that augments the $A \beta$ aggregation induced pathogenesis, are the drugs of abuse $[147, \underline{148}]$. These powerfully addictive stimulant drug molecules have been studied to have an exaggerating effect during HIV infection [149]. Cocaine (coc), a very common abused drug within PLWH, exerts malicious effects on the CNS [150-152]. Therefore, our hypothesis is based on the concept that in the presence of coc, the additive effect of HIV-

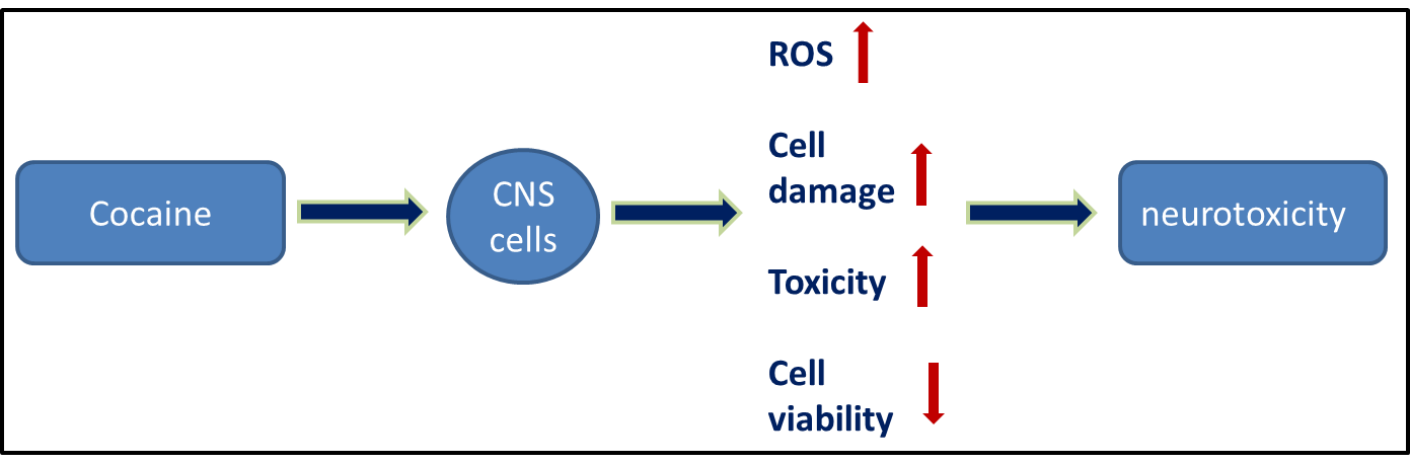

Figure 2.2 Cocaine and $A \beta 40$ secretion

Cocaine has a short half-life of only about an hour but its effects, predominantly on the CNS is strong, it may contribute in Cell damage and toxicity, and also increase the oxidative stress, therefor contributing more towards disease pathogenesis. 
1 Tat and coc may increase $A \beta$ aggregation, which is a common factor in aging and HIV associated neurological disorders. Cocaine has a short half-life of only about an hour but its effects, predominantly on the CNS, is strong and deteriorating (Figure 2.2). Therefore, investigating therapies for targeting coc abusing aging PLWH population is one of the main requirements to manage neurological disorders.

In this chapter, we have focused on elucidating the therapeutic properties of Withaferin A (WA) against multiple disease-associated factors including A, HIV-1 Tat and drug of abuse, coc. WA is an active purified drug moiety extracted from ASH, isolated from the root extract of a medicinal plant Withania Somnifera $[119,120]$. WA is a steroidal lactone, by its chemical nature, and its parent compound ASH has been traditionally used in ayurvedic medicine from ancient times in countries like India. WA is the first member of the withanolide class of ergostane type product to be discovered [153]. The beneficial effects of WA has been studied in the field of tumor inhibition [154], antiangiogenic activity [155-157], and against angioproliferative and malignant diseases like pancreatic cancer [158], leukemia, breast cancer and colon cancer [159]. It is also studied for its antimetastasis [160] and anti-carcinogenic properties [161]. WA is also explored in the field of apoptosis and adipogenesis inhibitor in 3T3-L1 adipocytes [162]. However, the therapeutic ability of WA against neurological disorders, is not well studied yet. Therefore, according to our hypothesis, we propose that WA is a neuroprotectant, which reduces $A \beta 40$ induced toxicity in human neuroblastoma cell lines in-vitro. Therefore, we planned a systematic study, to explore for the first time the neuroprotective role of WA against $A \beta$ secretion and aggregation, and exaggerating effects of HIV-1 Tat and Coc, in-vitro. 


\subsection{Methodology}

\subsubsection{Chemicals and Reagents}

WA was commercially purchased from Sigma Aldrich (Cat\# W4394 SIGMA). Methylthiazolyldiphenyl-tetrazolium bromide (MTT; Cat\# M2003) and paraformaldehyde was purchased from Sigma Aldrich. HIV-1 clade B recombinant Tat protein (86-amino acid) was obtained from NIH AIDS research and reference reagent program (Cat\# 2222).

\subsubsection{Cell Culture}

The cell type used in this study are SHAPP which is a human neuroblastoma cell line stably over-expressing human APP751 (kind gift from Dr. Jonathan Geiger, University of North Dakota, Grand Forks, ND, USA). SH-APP cells were cultured in Dulbecco Eagle's minimum essential medium (DMEM; Gibco®; Life Technologies, Grand Island, NY, USA) supplemented with $10 \%$ fetal bovine serum, $100 \mathrm{U} / \mathrm{ml}$ penicillin/streptomycin, nonessential amino acids, and sodium pyruvate $(1 \mathrm{mM})$ at $37^{\circ} \mathrm{C}$ in $5 \% \mathrm{CO} 2$.

\subsubsection{Cell Viability Assay}

SHAPP cells were plated at a density of $1 \times 10^{4}$ cells per well into 96 -well plates and maintained at $37^{\circ} \mathrm{C}$ for $24 \mathrm{hrs}$. Cells were treated with various concentrations of WA for 48 hrs. Fresh medium containing $50 \mu \mathrm{L}$ of MTT solution $(0.5 \mathrm{mg} / \mathrm{mL})$ was added to each well. After 3hrs of incubation, the MTT formazan crystals were dissolved in dimethyl sulfoxide (DMSO) and viable cells were detected by measuring the absorbance at $570 \mathrm{~nm}$ using a microplate reader (Molecular Devices, Sunnyvale, CA, USA).

For Tat and coc toxicity study on cell viability, we performed cell viability test using $0.4 \%$ Trypan Blue Solution (T8154) live-dead screening. 10 $\mu$ l of cells were taken from the pellet 
resuspended in fresh media, after centrifugation at $1500 \mathrm{rpm}$ for $5 \mathrm{mins}$, and was mixed with $10 \mu$ l of Trypan blue dye (1:1 ratio). The cells were then loaded on a cell counting slide and counted for live count on a cell counter (BioRad TC20 ${ }^{\mathrm{TM}}$ Automated cell counter).

\subsubsection{Tat and Coc Treatment of SH-APP Cells}

SHAPP cells $\left(1 \times 10^{6}\right.$ cells) were cultured overnight in T-25 flasks in complete DMEM media. After $48 \mathrm{hrs}$, the SHAPP cells were treated with different concentrations of HIV-1 Tat $(5-100 \mathrm{ng} / \mathrm{ml})$ and $\operatorname{coc}(0.1-10 \mu \mathrm{M})$ and the cells and supernatant were collected after $48 \mathrm{hrs}$ after the treatment. The optimized dose of Tat and coc were selected based on their effect on increasing $A \beta$ levels significantly compared to untreated controls. In further experiments, $1 \times 10^{5} \mathrm{SH}$-APP cells were seeded in six wells plates and were cultured for $48 \mathrm{hrs}$. Cells were treated with optimized concentrations of HIV-1 Tat1-72 and/or coc.

\subsubsection{Quantification of $A \beta 40$ Levels}

Secreted $A \beta$ levels were measured using human A $\beta 40$ ELISA kit as per the manufacturer's protocol (Thermo Fisher Scientific, Catalog\# KHB3481). For secreted A $\beta$ measurements, SH-APP cells were cultured in six well plates and after $48 \mathrm{~h}$, cells were treated with HIV$1 \mathrm{Tat} / \mathrm{coc}$ in combination with WA. After 24,48 and 72 hours the supernatant from cultured cells were collected and protease inhibitor was added. The supernatant was analyzed by human A 340 ELISA kit, as per the specific reagents and protocol provided with the kit. Each sample was analyzed in duplicate. Cells were used for flow cytometry studies to estimate intracellular A $\beta 40$ level. Additionally, for studies including Tat and coc, we added Tat and Cocaine to the SHAPP cells ( $80 \%$ confluent) and then after 24 hours, WA was added to the wells (in Tat+WA and Coc+WA wells). Since WA showed best efficacy at 48 hours (established from our previous study), we collected supernatant after 48 hours to be analyzed by A 340 ELISA. 


\subsubsection{Flow Cytometry}

Cells treated with various combinations including Tat alone, coc alone, Tat+/-WA, Coc+/WA were analyzed by flow cytometry studies to demonstrate changes in cellular $A \beta 40$ levels, in SH-APP cells after treatment with various concentrations of WA, Tat and coc. $1 \times 10^{6} \mathrm{SH}$-APP cells were stained with primary anti-human A 340 (\#PA3-16760) and secondary anti-rabbit Fluorescein isothiocyanate (FITC)-labeled antibody (catalog \#AP187F, Millipore). Auto fluorescence of the cells was based on the unstained cells. Cells were gated based on the secondary antibody. Accuri BD flow and Amnis ${ }^{\circledR}$ Imaging Flow Cytometers were used for acquisition. Analysis was conducted by Flow Jo software.

\subsubsection{Single-Cell Flow Cytometry}

The SHAPP cells were treated with different concentrations of WA and harvested after 48hrs of treatment, washed and counted. Equal amounts of cells $(1 \times 106)$ were aliquoted in $1.5 \mathrm{ml}$ Eppendorf centrifuge tubes in $250 \mu \mathrm{l} 1 \mathrm{X}$ PBS. Cells were analyzed by ImageStreamX Imaging Flow Cytometer (Amnis Corporation, Seattle, WA, USA). A magnification of 60X was employed for all readings. Ten-thousand cells (events) were analyzed for each sample. FITC and DAPI were excited with a $100 \mathrm{~mW}$ of $488 \mathrm{~nm}$ argon laser. FITC and DAPI fluorescence was collected on channel two (505-560 nm) and channel seven (560-595 nm), respectively. Intensity adjusted bright field images were collected on channel one. Bright field area and total fluorescence intensity were calculated using IDEAS software. Data analysis was performed using the IDEAS software (Amnis Corporation), with proper data compensation with respect to singly stained samples. The compensated data was then gated to eliminate cells that were out of field of focus and doublets or debris was eliminated too. 


\subsubsection{Immunofluorescence Staining and Analysis for Studying Beta Amyloid Aggregation}

To study the effect of WA on the morphology aggregation, we conducted immunofluorescence imaging experiment. The cells were cultured to $80 \%$ confluence on the 4-well microscopy slides and were then exposed to HIV-1 Tat+/- WA. After 48hrs, the supernatant was discarded and the cells were fixed in 4\% PFA. PFA embedded slides were then immunostained by using $A \beta 40$ primary antibody (1:100) and GFP secondary antibody (1:100). Immunohistochemically stained sections were captured using the Keyence microscope. The images were captured at a magnification of 10X.

\subsubsection{Immunofluorescence Staining for Studying Effect of WA on Neuronal Morphology}

To study the effect of WA on the neuronal morphology, we conducted immunofluorescence-imaging experiment. The cells were cultured to $80 \%$ confluence on the 4-well microscopy slides and were then treated with coc +/- WA. After 48hrs, the supernatant was discarded and the cells were fixed in 4\% PFA. PFA embedded slides were then washed and immunostained using MAP2 primary antibody (1:100) and antiFITC secondary antibody (1:100).

\subsubsection{Congo red staining for staining $A \beta$}

SH-APP cells were grown in 2 chamber slides at a concentration of $5.0 \times 10^{3} / \mathrm{ml}$ for 48 hrs. The cells were then treated with optimized concentration of WA $(1 \mu \mathrm{M})$ for further 48 hrs. Cell culture supernatant was discarded and cells were stained for Congo-red. For WA additions, DMSO served as the vehicle to dilute the compound at a final concentration of 
$1 \mu \mathrm{M}$. Control cultures were left untreated. After 48 hours, cells were washed with PBS, fixed in $4 \%$ formalin for $15 \mathrm{~min}$ at room temperature. Again cells were washed with PBS and then stained with a fresh alkaline solution of $0.5 \%$ filtered Congo red (Sigma-Aldrich) at room temperature for $5 \mathrm{~min}$. The cells were then washed with deionized water carefully, and the slides were mounted in DAPI Fluoromount-G® (Southern Biotech, Catalogue No. 0100-20) and then observed through Keyence microscope. The images were captured at a magnification of $10 \mathrm{X}$.

\subsubsection{Beta secretases ELISA}

The effect of WA on the $\beta$ secretases was studied in-vitro by measuring $\beta$ secretases levels using BACE-1 human ELISA kit as per the manufacturer's protocol (Thermo Fisher Scientific, Catalog\# EHBACE1). For $\beta$ secretases measurements, SH-APP cells were cultured in six well plates and after $48 \mathrm{hrs}$, cells were treated with WA compared to untreated controls. The supernatant from cultured cells was collected and protease inhibitor was added to it. The supernatant was analyzed for their $\beta$ secretase levels. Each sample was analyzed in duplicate, and was repeated three times.

\subsubsection{Gamma secretases ELISA}

The effect of WA on the Gamma secretases was studied by ELISA. Y secretases levels were measured using $\mathrm{y}$ secretase human ELISA kit as per the manufacturer's protocol (My bio Source, Catalog\# MBS704513). For y secretases measurements, SHAPP cells were cultured in six well plates and after $48 \mathrm{hrs}$, cells were treated with WA compared to untreated controls. The supernatant from cultured cells was collected and protease inhibitor was added to it. The supernatant was analyzed for their y secretase levels. Each sample was analyzed in duplicate. 


\subsubsection{Data Analysis}

Results in this study are representative of three or more independent experiments. Statistical significance was analyzed using Graph Pad Prism5 software, La Jolla, CA, USA by performing ANOVA or the Student's $t$-test for unpaired observations. The values are presented as mean \pm SEM.

\subsection{Results}

\subsubsection{WA dose optimization and A $\beta$ decreasing efficacy studies in SH-APP cells}

To optimize the non-toxic dose of WA, different concentrations of WA $(0.5-10 \mu \mathrm{M})$ were treated to SH-APP cells. The dose-dependent and time-dependent (data not shown) ELISA study demonstrated that $2 \mu \mathrm{M}$ concentration of WA at 48 hours reduces the secreted AB40 in SH-APP cells significantly when compared to non-treated control, (Figure 2.3 A) without causing cytotoxicity (Figure 2.3 B). Further, results were confirmed with the flow cytometry and showed (Figures 2.4 A-C) dose dependent reduction in the $A \beta 40$ levels and the maximum reduction was reported at $2 \mu \mathrm{M}$ WA concentration without causing cellular toxicity. Additional single cell flow cytometry and imaging also showed the same trend highlighting the effective role of $2 \mu \mathrm{M}$ WA against $A \beta 40$ (Figures $2.5 \mathrm{~A}, \mathrm{~B}$ ). 

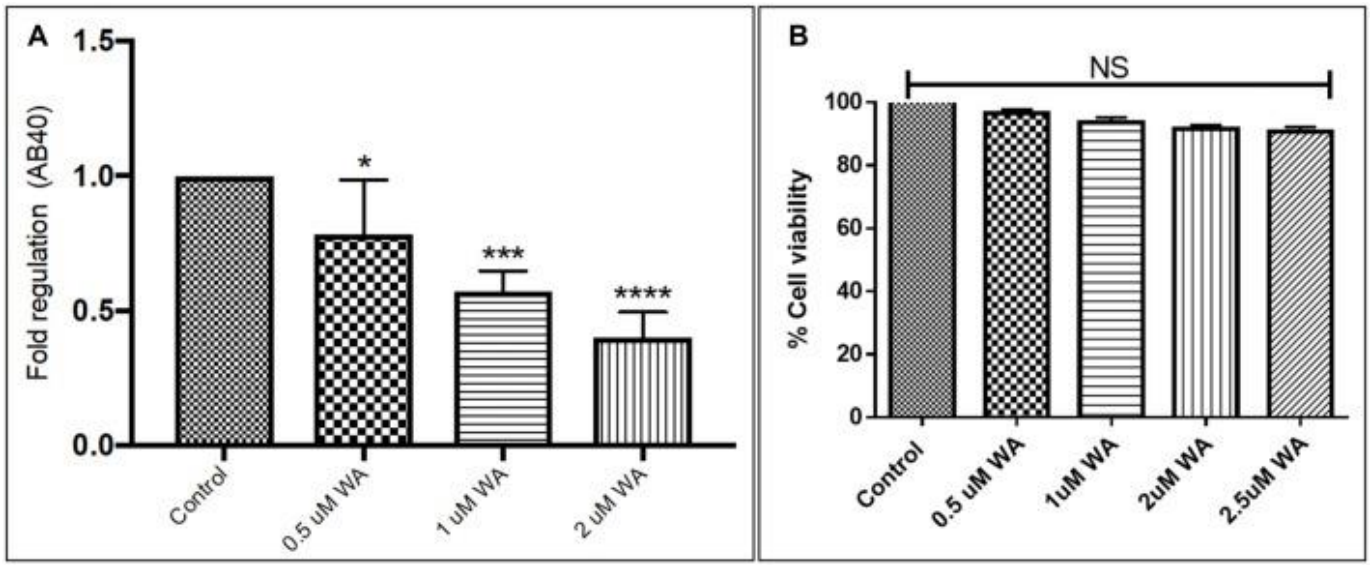

Figure 2.3 Effect of Withaferin A (WA) on amyloid beta (A $\beta$ ) secretion

(A) Cells were treated with different dose of WA and the supernatants were collected $48 \mathrm{~h}$ after treatment. The supernatant collected were analyzed by A 340 ELISA that demonstrated that at an optimum dose of $2 \mu \mathrm{M} \mathrm{WA}$, the levels of secreted $A \beta 40$ showed significant decrease compared to control untreated samples. (B) The dosage of WA used for this experiment were also analyzed for the associated cellular toxicity. The cell toxicity assay showed that the lower doses of WA were not toxic to cells. Optimum dose of $2 \mu \mathrm{M}$, WA did not cause any loss in cell viability or toxicity in SHAPP cells. 


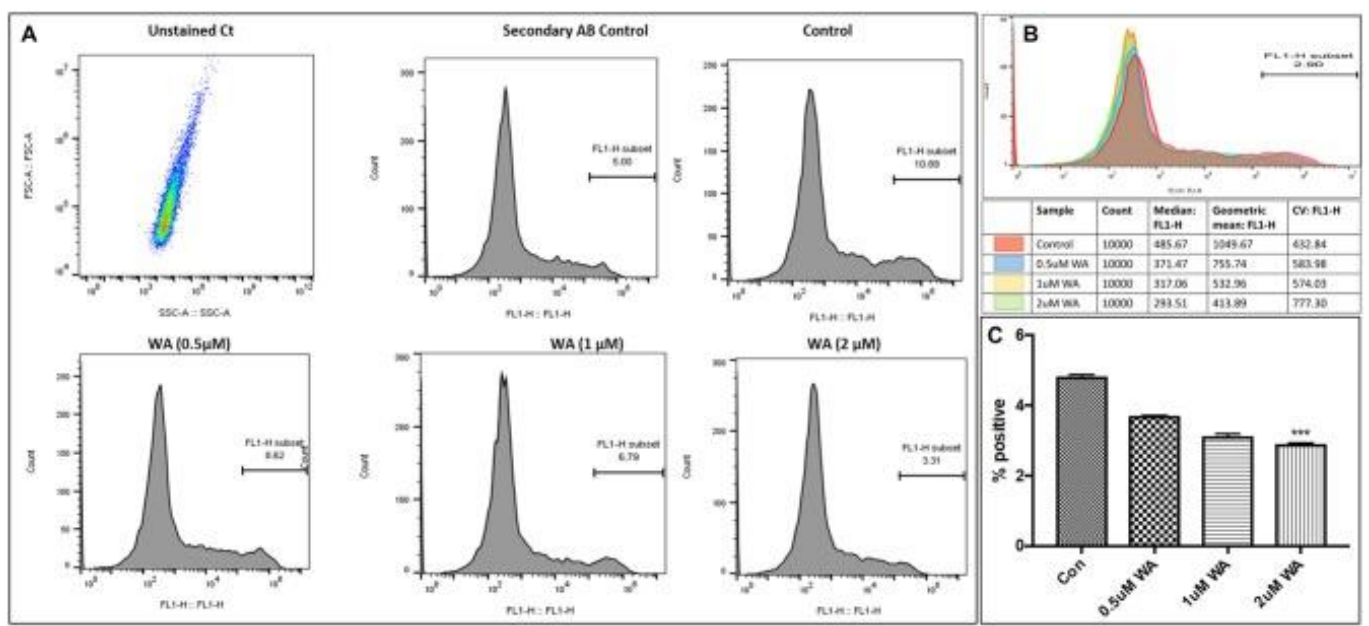

Figure 2.4 WA inhibits $A \beta 40$ in concentration dependent manner

Panel (A) shows histograms of $A \beta$ secretion by the SHAPP cells upon treatment with varying dose of WA. Panel (B) shows the layover of the peaks in one histogram, and $(C)$ shows the quantification of the same. The cells were treated with WA concentrations, and after $48 \mathrm{hrs}$ of treatment were analyzed by Flow cytometry for determining $A \beta 40$ levels. Flow cytometry was used to demonstrate the expression of $A \beta 40$ in SH-APP cells after treatment with three different concentrations of WA. $1 \times 10^{6} \mathrm{SH}$-APP cells were stained with primary anti-human AB40 (\#PA3-16760) and secondary anti-rabbit Fluorescein isothiocyanate (FITC)-labeled antibody (catalog \#AP187F, Millipore). Auto fluorescence of the cells was based on the unstained cells. Cells were gated based on the secondary antibody. Accuri BD flow and Amnis ${ }^{\circledR}$ Imaging Flow Cytometers were used for acquisition. Analysis was conducted in Flow Jo software and Amnis ${ }^{\circledR}$ FlowSight ${ }^{\circledR}$ Imaging Flow Cytometer and analysis by IDEAS $\AA$ Image software. For each experiment, from all events collected, FITC positive cells were gated from single cells. 

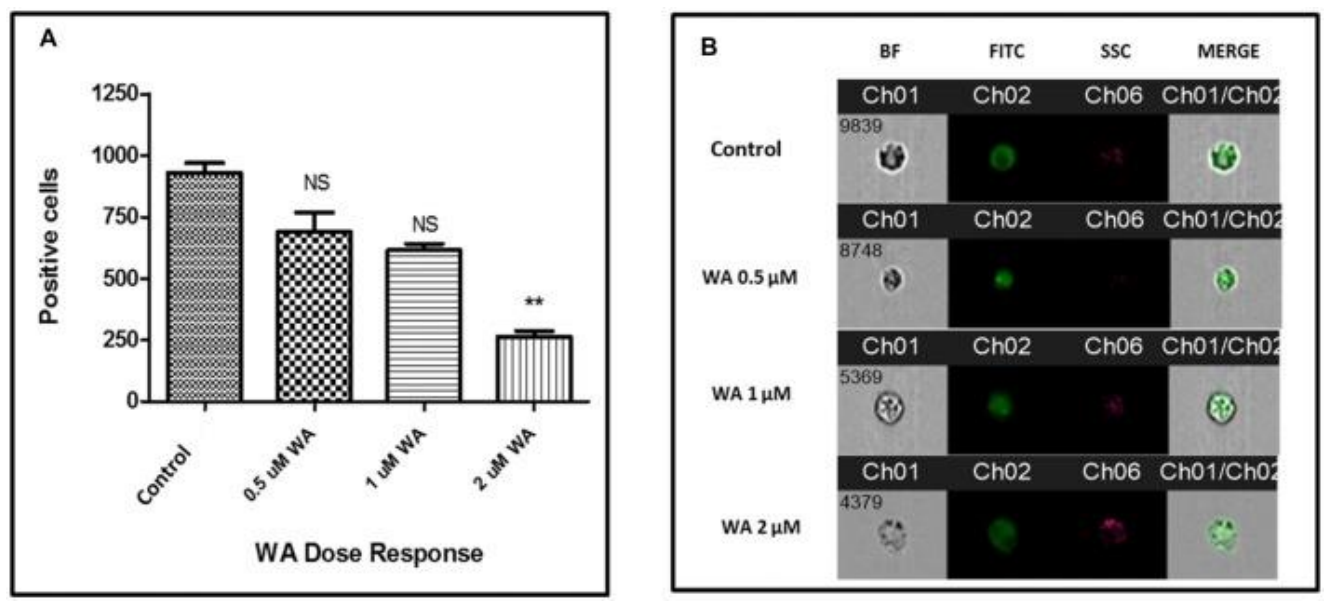

Figure 2.5 WA inhibits $A \beta$ production

Single cell flow Cytometry was used to identify the expression of $A \beta 40$ protein in SHAPP cells after treatment with different concentration of WA. (A) Bar graph representing the mean \pm standard error of percent of mean fluorescence intensity. (B) Representative single cell images. We have observed significantly reduced $A \beta$ with WA exposure dose dependently.

\subsubsection{Effect of HIV-Tat protein on A $\beta$ production in SH-APP cells}

Human A $A 40$ ELISA was performed with the supernatant collected from control and WA treated SHAPP cells to evaluate the efficacy of WA in reducing the HIV-Tat and coc induced increase in A 340 levels. SHAPP cells were treated with different concentrations of Tat $(5-50 \mathrm{ng} / \mathrm{ml})$. Figure 2.6 shows that the SHAPP cells treated with Tat exhibited upregulation of $A \beta 40$ secretion compared to untreated control (Figures 2.6 $A, B$ ). Effective dose of Tat $(50 \mathrm{ng} / \mathrm{ml})$ when treated with $2 \mu \mathrm{M}$ WA, showed significant decrease in $A \beta 40$ (Figure 2.6C). Further, the results were also confirmed by the flow cytometry using $A \beta 40$ specific primary antibody. The dose of $50 \mathrm{ng} / \mathrm{ml}$ Tat most significantly increased the A levels when compared to control (Figure 2.7 A,B). 

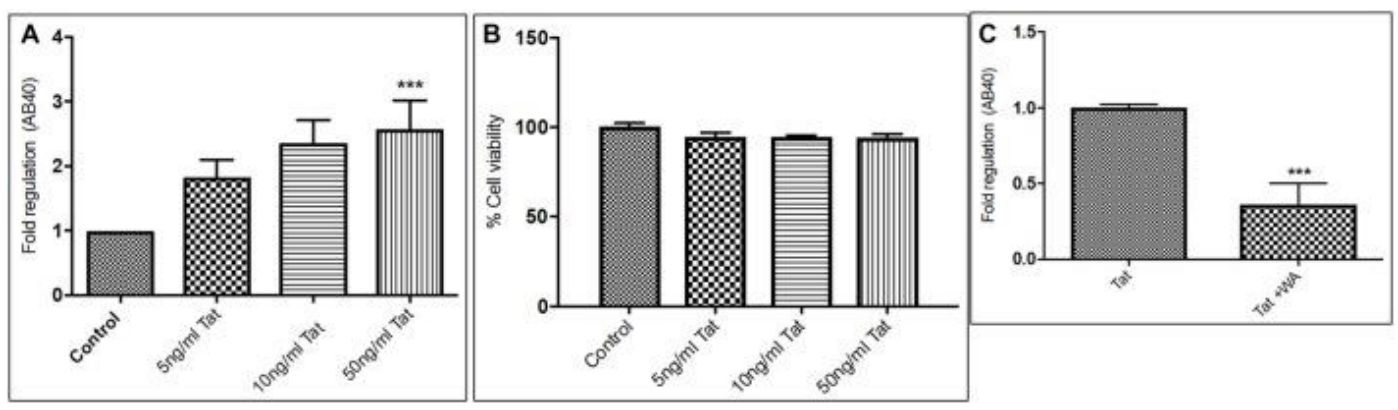

Figure 2.6 Tat induces increase in secreted $A \beta 40$ levels

(A). Human A $\beta$ ELISA analysis showing that Tat $(5-50 \mathrm{ng} / \mathrm{mL})$ increased the secreted A 40 significantly in SHAPP cells. (B) Cellular toxicity assay showing viability of the cells in the Tat treated samples. (C) $2 \mu \mathrm{M}$ WA reduced the Tat levels significantly when compared to Tat $(50 \mathrm{ng} / \mathrm{mL})$ only treated samples. $1 \times$ $10^{6}$ SHAPP cells were seeded in 6-well plates and were grown for 48hrs and then treated with Tat protein in different doses and the cells were then incubated for $48 \mathrm{hrs}$ at $37^{\circ} \mathrm{C}$. The supernatant from the culture was collected and treated with protease inhibitor $(1 \mu \mathrm{l} / \mathrm{ml})$ and analyzed by A 40 ELISA (Sigma). The results are from three independent experiments and the statistical significance was calculated by Student's t-test. Cell viability study was performed by Trypan blue live dead screening, to study the toxicity levels of various Tat dose. Dose selected for Tat treatment for further experiment was elected based on increase in $A \beta 40$ secretion levels and correlated with cell viability. 


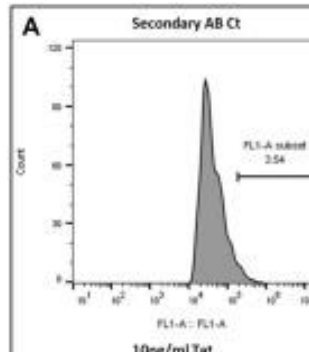

$10 \mathrm{ceg} / \mathrm{mi}$ Tat

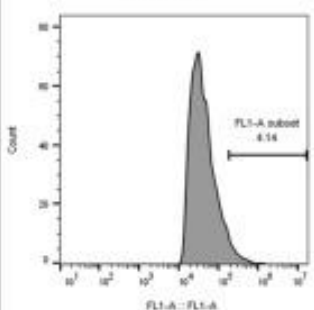

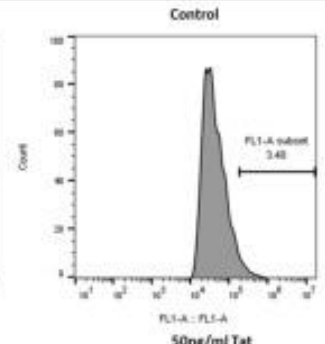

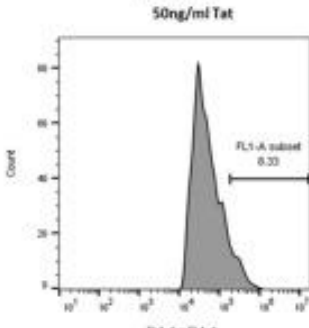

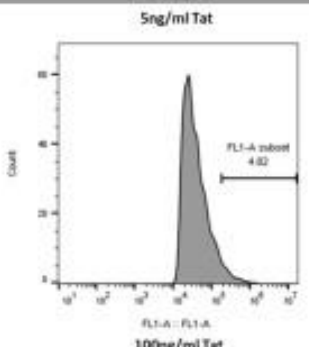

$100 \mathrm{ng} / \mathrm{mi}$ Tat

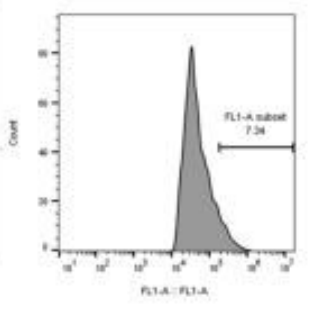

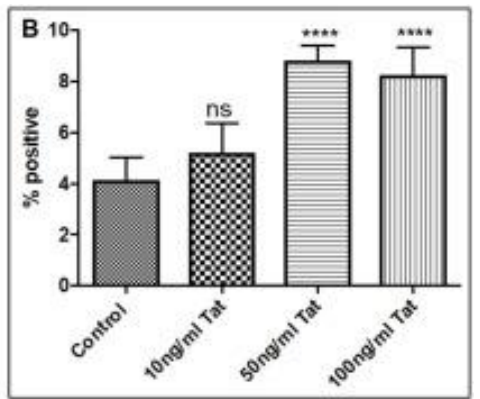

Figure 2.7 Dose response

(A) Histograms showing Tat $(5-100 \mathrm{ng} / \mathrm{mL})$ increases the A $\beta 40$ levels. SHAPP cells were treated with different concentrations of Tat and after 48hrs of treatment were analyzed by Flow cytometry for studying the cellular A 440 levels. (B) Quantification representation of the percent positive cells.

\subsubsection{Effect of HIV-Tat protein and Cocaine on A $\beta$ production in SH-APP cells}

Additionally, Human A 440 ELISA was performed with the supernatant collected from control and WA treated SHAPP cells, to evaluate the efficacy of WA in reducing the coc induced $A \beta$ secretion. SHAPP cells were treated with different concentrations of $\operatorname{coc}(0.1-$ $10 \mu \mathrm{M})$. We studied the effect of coc in the similar study pattern, and observed the increase in $A \beta 40$ secretion (Figures $2.8 \mathrm{~A}, \mathrm{~B}$ ). We report that $0.1 \mu \mathrm{M} \operatorname{coc}$ showed most significant up-regulation in $A \beta 40$ levels compared to untreated controls. Effective dose of $\operatorname{coc}(0.1 \mu \mathrm{M})$

when treated with $2 \mu \mathrm{M}$ WA, showed significant decrease in A 340 (Figure $2.8 \mathrm{C}$ ). This result was further confirmed by the flow cytometry studies which showed a coc induced increase in $A \beta$ levels (Figures $2.9 \mathrm{~A}, \mathrm{~B}$ ). 


\subsubsection{Tat and coc Induced Increase in A 440 Levels, in combination}

An optimized dose of Tat $(50 \mathrm{ng} / \mathrm{mL})$ and $\operatorname{coc}(0.1 \mu \mathrm{M})$ alone or in combination were used to study the decreasing efficacy of WA $(2 \mu \mathrm{m})$ in SH-APP cells. Results showed the
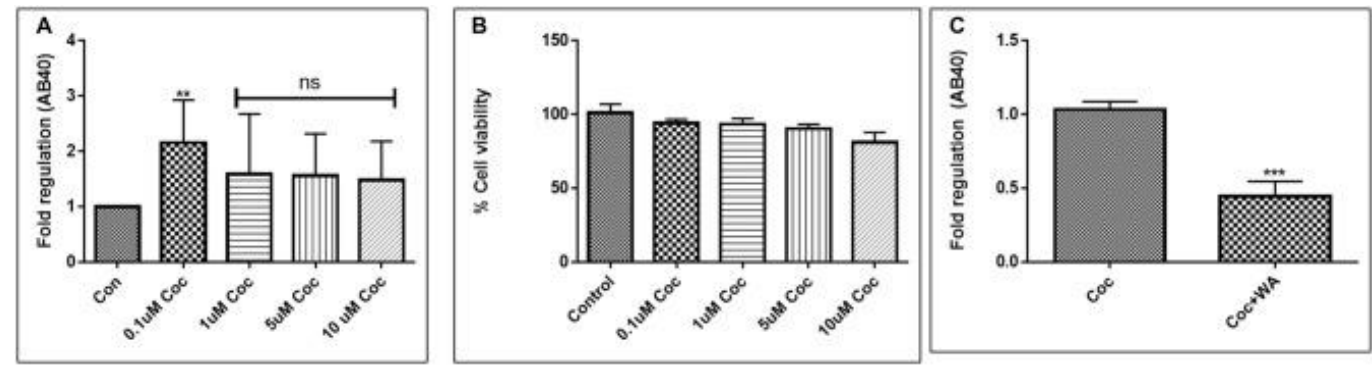

Figure 2.8 Coc induces increase in secreted $A \beta 40$ levels

Similar study pattern of ELISA and flow cytometry, like in the case of HIV-1 Tat, was performed with various concentrations of coc to choose an optimized dose of coc for further studies. (A) Coc increases $A \beta 40$ secretion. Coc $(0.1-$ $10 \mu \mathrm{M})$ increased the secreted $A \beta 40$ but the significant increase was found in the samples treated with $0.1 \mu \mathrm{M} \operatorname{coc}$. (B) Cellular toxicity assay showing viability of the cells in the coc treated samples. (C) $2 \mu \mathrm{M}$ WA reduced the coc $(0.1 \mu \mathrm{M})$ induced $A \beta 40$ levels significantly when compared to coc only treated samples.

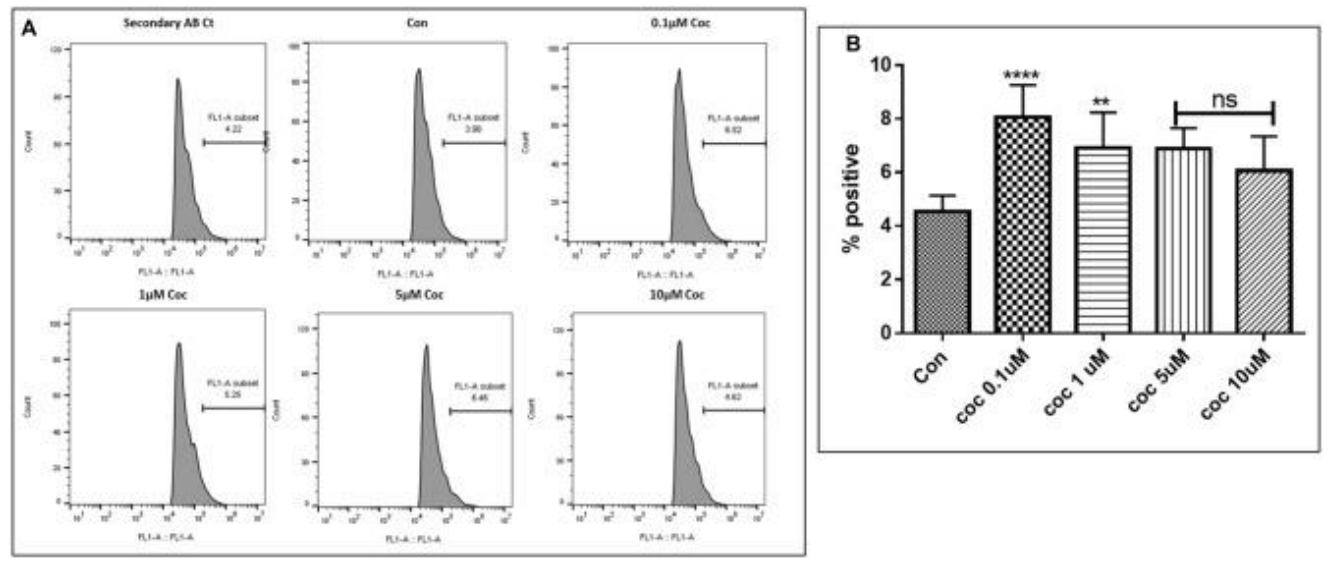

Figure 2.9 Dose response

(A) Histograms showing $\operatorname{coc}(0.1-10 \mu \mathrm{M})$ increases the $A \beta 40$ levels. The cells were treated with different concentrations of $\operatorname{coc}$ and after $48 \mathrm{~h}$ of treatment were analyzed by flow cytometry for determining the $A \beta 40$ levels. (B) Quantification representation of the percent positive cells $\left({ }^{* *} \mathrm{p} \leq 0.01 ;{ }^{* * * *} \mathrm{p} \leq\right.$ 0.0001; ns, not significant). 
combined effect of Tat and coc together in increasing the $A \beta 1-40$ levels. (Figures 2.10 A,B). Individual optimized dose of Tat $(50 \mathrm{ng} / \mathrm{mL})$ and $\operatorname{coc}(0.1 \mu \mathrm{M})$ were used for further WA decreasing efficacy studies.
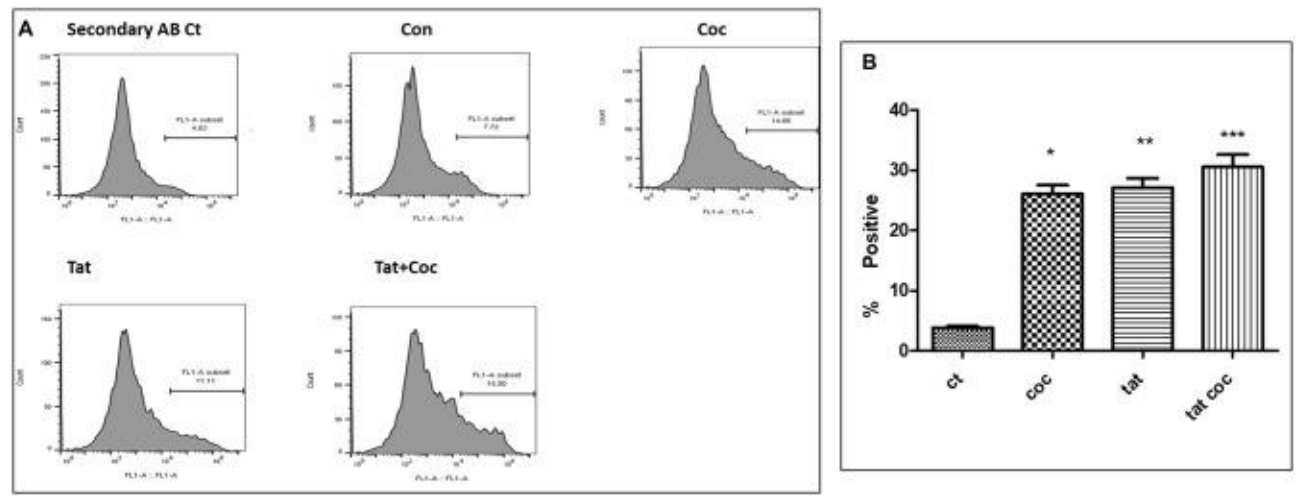

Figure 2.10 Effect of Tat and coc combination.

(A) Tat $(50 \mathrm{ng} / \mathrm{mL})$ and $\operatorname{coc}(0.1 \mu \mathrm{M})$ individually and in combination increase the $A \beta 40$ levels. The cells were treated with selected Tat and coc concentrations and combination of both, and after 48hrs of treatment were analyzed by flow cytometry for determining the $A \beta 40$ levels. (B) Quantification representation of the percent positive cells.

\subsubsection{WA reverses Tat and Cocaine induced Amyloid aggregates in-vitro}

Immunocytochemistry studies showed that WA was able to reduce the amyloid aggregation when compared to the untreated control SHAPP cells. The cells were grown in the microscopic slides (eight wells) and after $24 \mathrm{hrs}$ of growth, the wells were treated individually with Tat+/- WA and coc+/- WA for 48hrs and control wells were replaced with fresh media. The cells were collected, fixed and stained with primary A $\beta 40$ antibody (1:100) and GFP secondary antibody (1:100). We observed that the cells exposed to Tat and $\operatorname{coc}$ had strong signals for $A \beta$ aggregation, which was mitigated by WA treatment as observed in the Tat+WA and coc+WA, when compared to control well (Figure 2.11 A-G). 

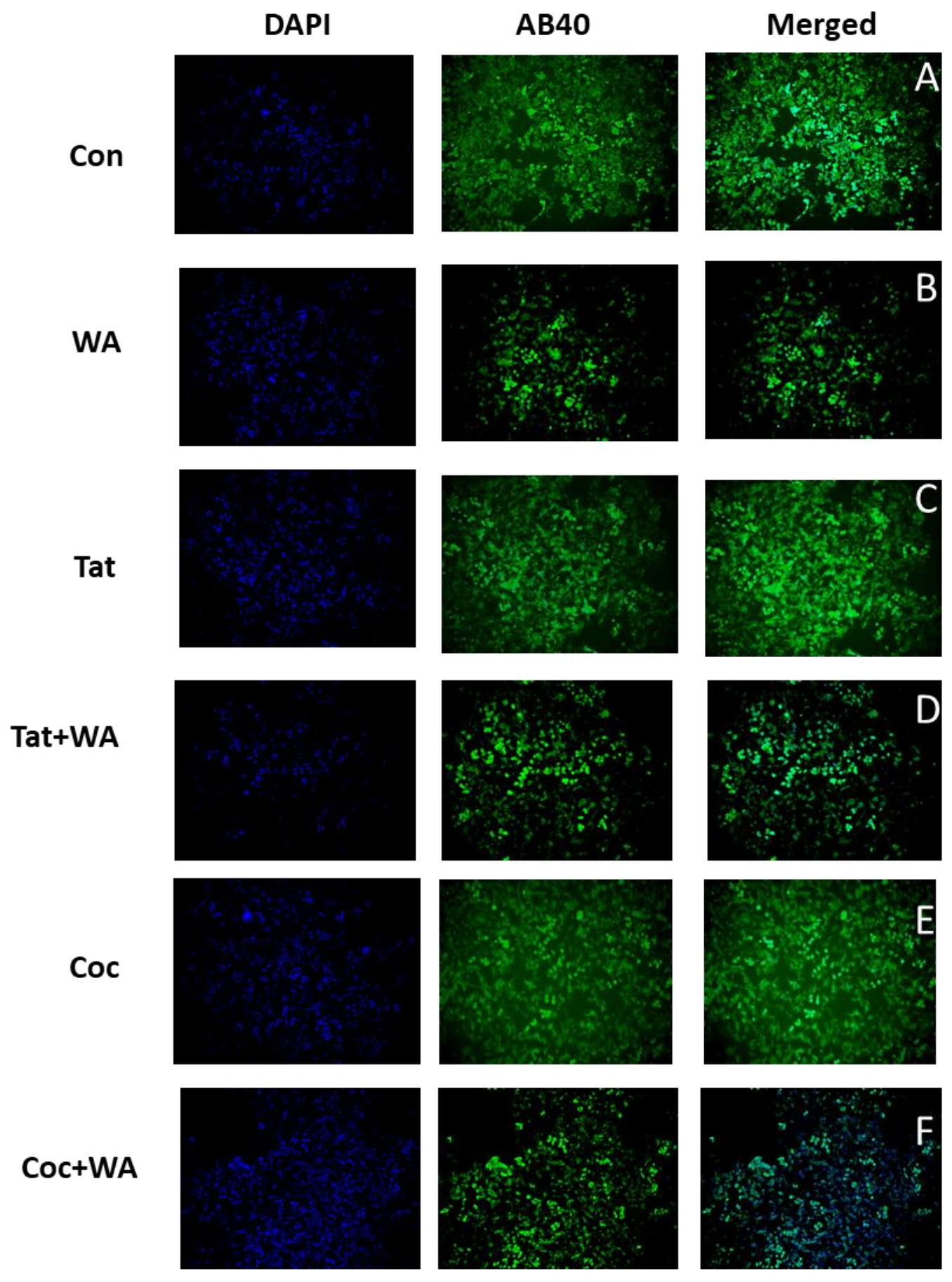


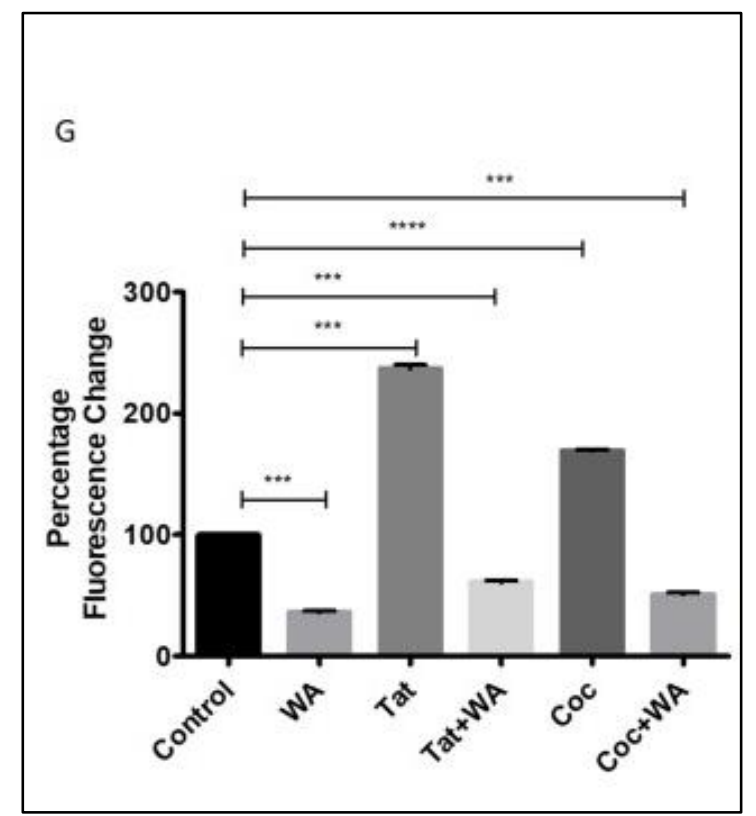

Figure 2.11 WA inhibits HIV-1 Tat induced A $\beta$-production, respectively SH-APP cells were treated with HIV-1 Tat $(50 \mathrm{ng} / \mathrm{mL}$; C) $\operatorname{coc}(0.1 \mu \mathrm{M}$; E) +/- WA $(2 \mu \mathrm{M} ;(\mathrm{D}, \mathrm{F})$ respectively) were compared to Control $(A)$ and only WA treated cells (B). After $24 \mathrm{~h}$, cells were fixed and stained with Anti-Human Aß40 overnight. Cells were washed and stained with secondary anti-rabbit FITC-labeled antibody (catalog \#AP187F, Millipore). Images were acquired using Keyence All in one microscope (10x). WA significantly suppressed Tat and coc induced $A \beta$-secretion, respectively. Florescent intensity of these stained cells was quantified using the ImageJ software $(G)\left({ }^{* * *} p \leq 0.001 ;{ }^{* * * *} p \leq 0.0001\right)$.

\subsubsection{WA may decrease Cocaine induced neurotoxicity}

To demonstrate the effects of coc and WA treatment on the SHAPP cells morphology, we conducted immunofluorescence imaging experiment. Cultures of SHAPP cells were grown in the chambered imaging slides for $48 \mathrm{hrs}$, and then were treated with coc+/-WA. The cells were allowed to grow and were then washed and stained with the neuronal marker anti-MAP2 primary antibody and then with anti-rabbit FITC-labeled antibody. We observed that the cells exposed to coc exhibited dendritic beading (indicated by yellow arrows) and cytoplasmic vacuoles (Figure 2.12 C) when compared to the untreated control 
(Figure 2.12 A) and WA only treated SHAPP cells (Figure 2.12 B) which showed no abnormal beading or thickening of the dendrites. Upon treatment with WA, in coc exposed cells (Figure 2.12 D), we observed reduced dendritic beading and more pronounced and elongated dendrites, communicating with other neuronal cells. We quantified the numbers of healthy dendrites (green) (excluding the distorted dendrites with dendritic beading) and number of vacuoles like distortions in the cell cytoplasm (purple). Our quantification demonstrated that WA treated coc exposed cells showed enhanced healthy dendrites and less vacuoles when compared to coc treated cells only (Figure 2.13). There were no
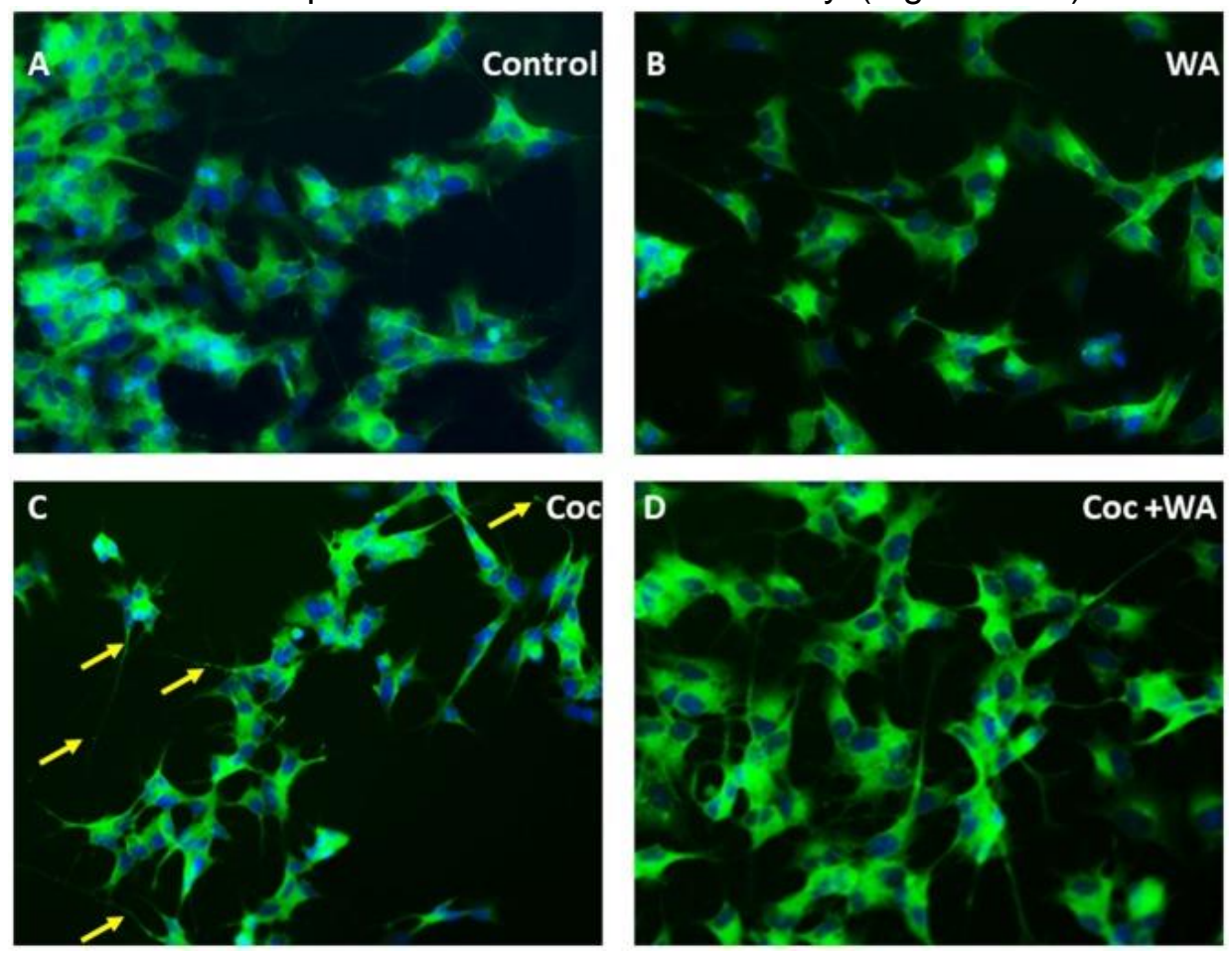

Figure 2.12. WA reverses coc induced dendritic beading and cytoplasmic vacuoles

SH-APP cells were treated with $\operatorname{coc}(0.1 \mu \mathrm{M})+/-$ WA $(2 \mu \mathrm{M})$. Cells were fixed and stained with MAP2 primary antibody overnight. Cells were washed and stained with secondary anti-rabbit FITC-labeled antibody. (A) Control SHAPP cells and (B) WA only treated cells showed no abnormal beading or thickening of the dendrites when compared to (C) coc exposed SHAPP cells which exhibited heavy dendritic beading (yellow arrows), measure of the cells being in drug-induced stress, (D) WA treated $\operatorname{coc}$ exposed cells on the other hand displayed reduced dendritic beading and elongated dendrites. 
significant changes observed in the number of cells with distorted cytoplasm between the different treatments. This indicates that coc induces the stressed environment in the cell culture system, which leads to neuronal damage and WA may reduce the damage caused by coc exposure.

A

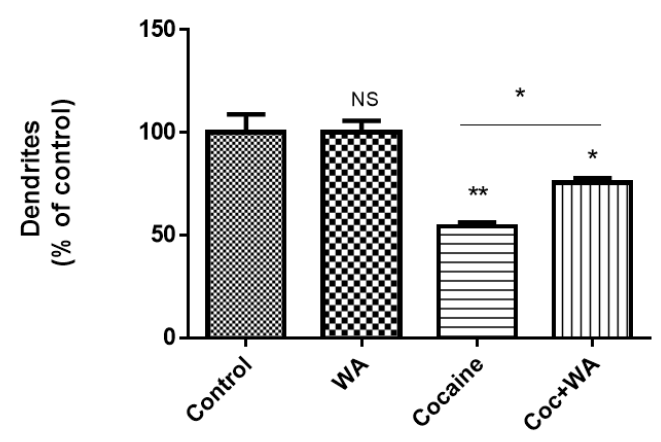

B

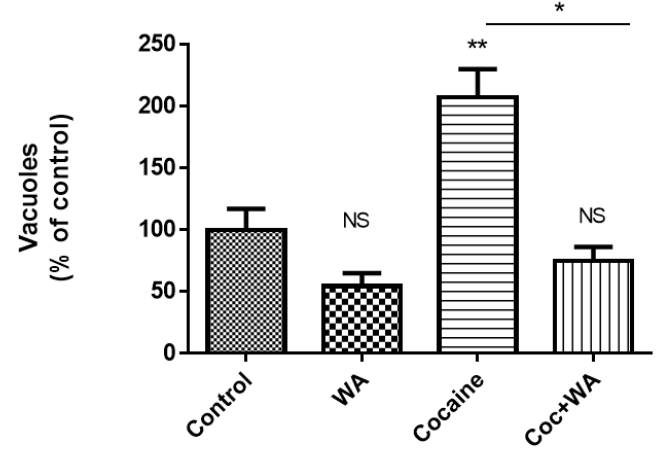

Fig 2.13 Quantification studies

Our quantification studies demonstrated that WA treated Coc exposed cells A) showed enhanced healthy dendrites and B) decreased vacuoles when compared to Coc treated cells only. There were no significant changes observed in the number of cells with distorted cytoplasm between the different treatments (The quantification is done from $n=4$ experiments). 


\subsubsection{Congo red stain based quantification of $A \beta$ in WA treated cells}

We studied the effect of WA on $A \beta$ by staining the cells with Congo red (CR) Stain. As Figure 2.14 (20X resolution) and Figure 2.15 (10X resolution) shows, cell cultures treated with WA alone showed reduced amyloid staining (specific to with the toxic A $\beta$ peptide) than in untreated SHAPP cell controls. CR also called as Amyloid stain was utilized for histological visualization of amyloid in the cell culture, which is also termed as indicator of amyloidosis (deposition of amyloid in tissues or cell culture). The principle behind the staining is that CR dye forms nonpolar hydrogen bonds with the amyloid peptides and red staining is visible when viewed by confocal microscopes due to the alignment of dye molecules with the linearly arranged amyloid fibrils. The high $\mathrm{pH}$ or alkaline environment improves the non-polar hydrogen bonding of $C R$ with the amyloid. CR tags the $A \beta$ peptide
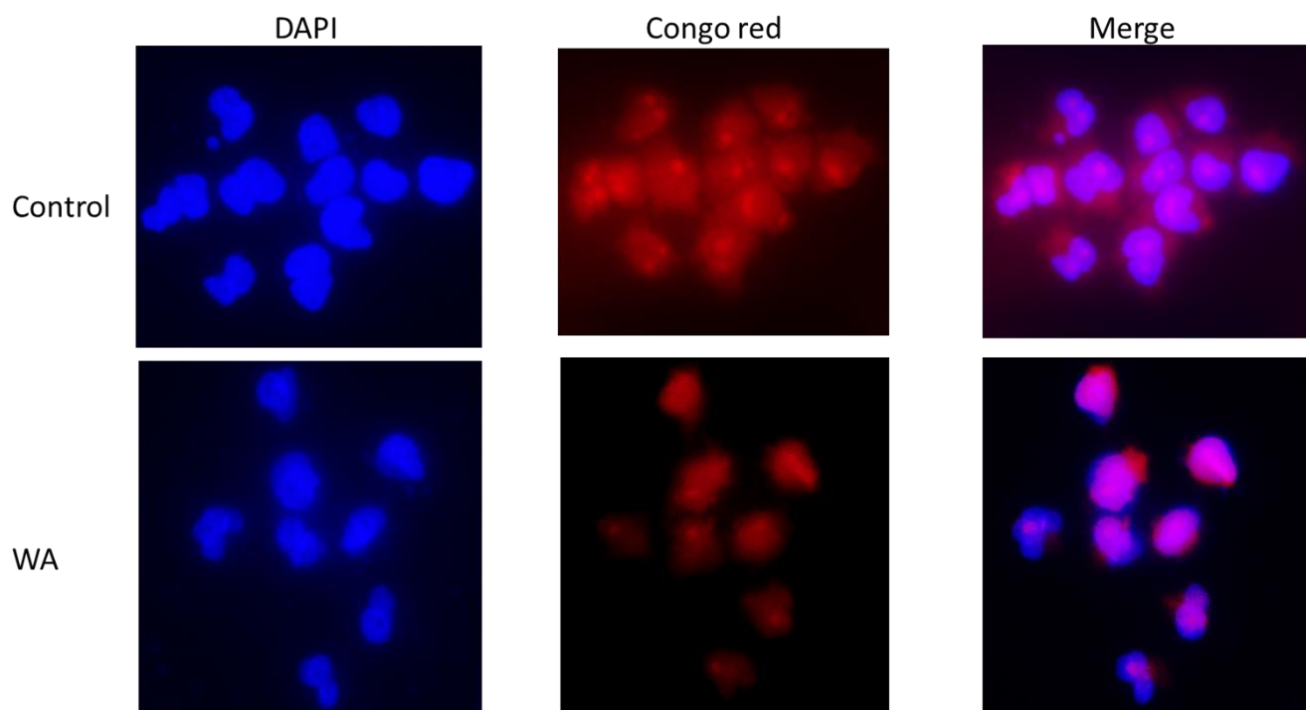

Figure 2.14 Congo red staining for Amyloid shows decreased amyloid in WA treated cells.

Control and WA treated SHAPP cells were stained by Congo red and observed by confocal microscopy; lens 20x. We observed that Cells treated with WA show less amyloid (red) compared to controls. Merged images show overall visualization of cell nuclei (blue), amyloid (red), and overlap (pink). Images are from one representative experiment of three independent experiments. 
and stains them red when untreated SHAPP cells expressing A $\beta$ were stained whereas cells treated with WA had reduced levels of $A \beta$, therefore less staining was observed in these control cells. We observed the cells at 10x and 20x resolutions. This study aligns with our prior results that WA has the ability to reduce $A \beta$ in-vitro.
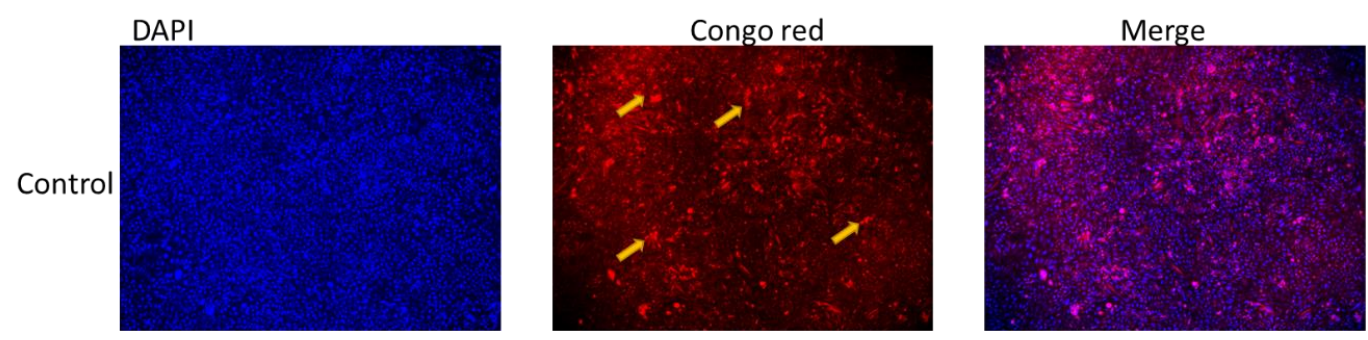

WA
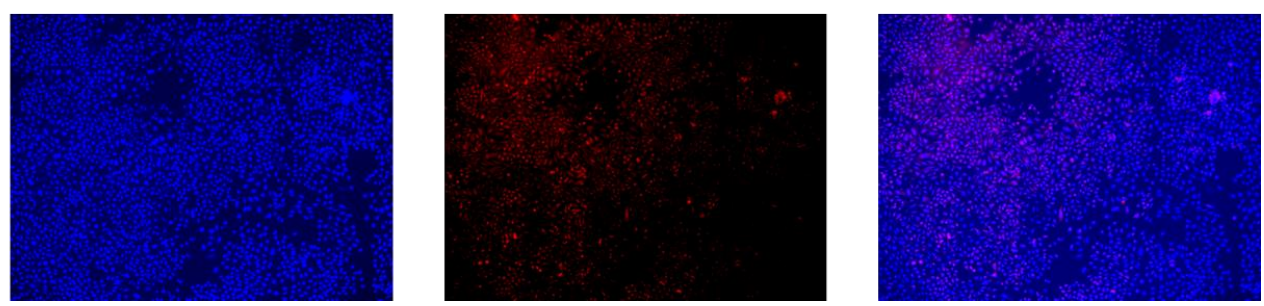

Figure 2.15 Congo red staining for amyloid shows decreased amyloid in WA treated cells.

Control and WA treated SHAPP cells were stained by Congo red and observed by confocal microscopy; lens 20x. We observed that Cells treated with WA show less amyloid (red) compared to controls. The mass pointed by yellow arrows are the aggregated amyloid. Merged images show overall visualization of cell nuclei (blue), amyloid (red), and overlap (pink). Images are from one representative experiment of three independent experiments.

\subsubsection{WA effect on Gamma secretases levels}

In order to understand the mechanism, behind WA's role against amyloid induced toxicity, we studied the effect of WA on the $y$ secretases. The human $y$ secretases ELISA study showed that WA did not have any effect on the level of $y$ secretase levels in the WA treated SHAPP cells when compare to untreated cells, in-vitro. An optimized dose of WA $(2 \mu \mathrm{M})$ 
was used to treat the SHAPP cells for 48 hours and the cell supernatant were used for $Y$ secretase ELISA. We found that the level of $y$ secretases in treated SHAPP cells remained same and showed no significant changes (Figure 2.16).
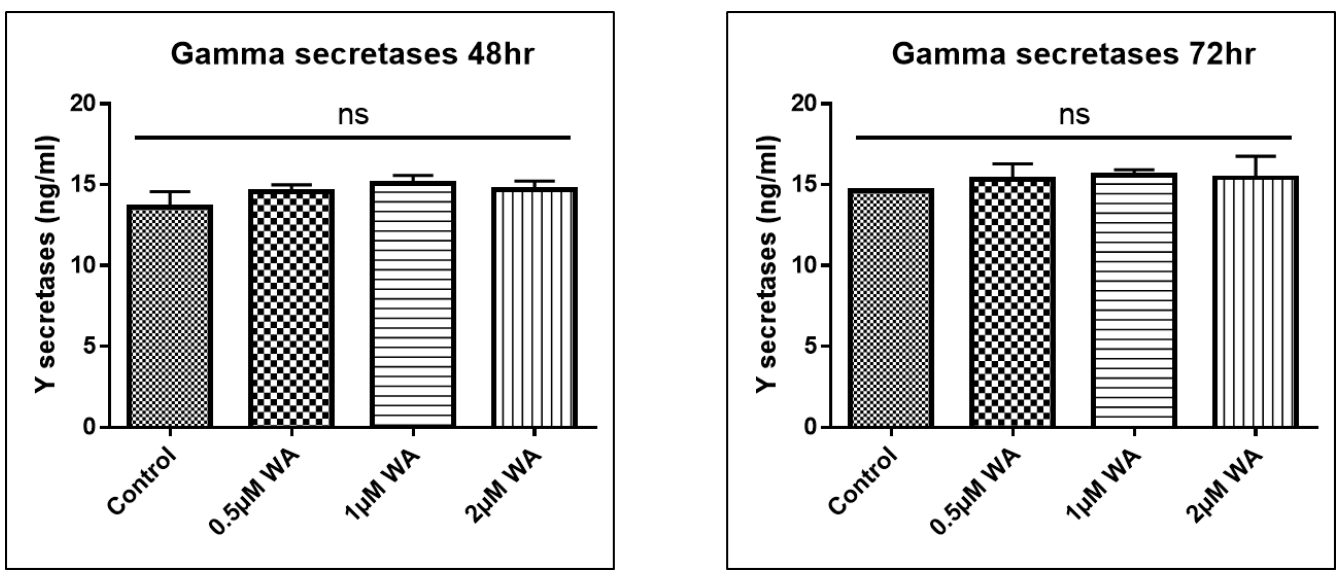

Figure 2.16 WA effect on the gamma secretases

No significant difference was observed in the levels of $y$ secretases in the supernatant from WA treated cells when compared to non-treated control.

\subsubsection{WA effect on Beta secretases levels}

We studied the effect of WA on the $\beta$ secretases. ELISA results demonstrated no change in the levels of $\beta$ secretases, in the WA treated cells when compared to control. An optimized dose of WA $(2 \mu \mathrm{m})$ was used to treat the SHAPP cells for $48 \mathrm{hrs}$ and the supernatant was then collected and used for the ELISA experiment. We found that WA was not able to reduce the levels of $\beta$ secretases in treated SHAPP cells, which could be due to low detection limit of the $\beta$ secretases from the supernatant samples in-vitro (Figure 2.17). 


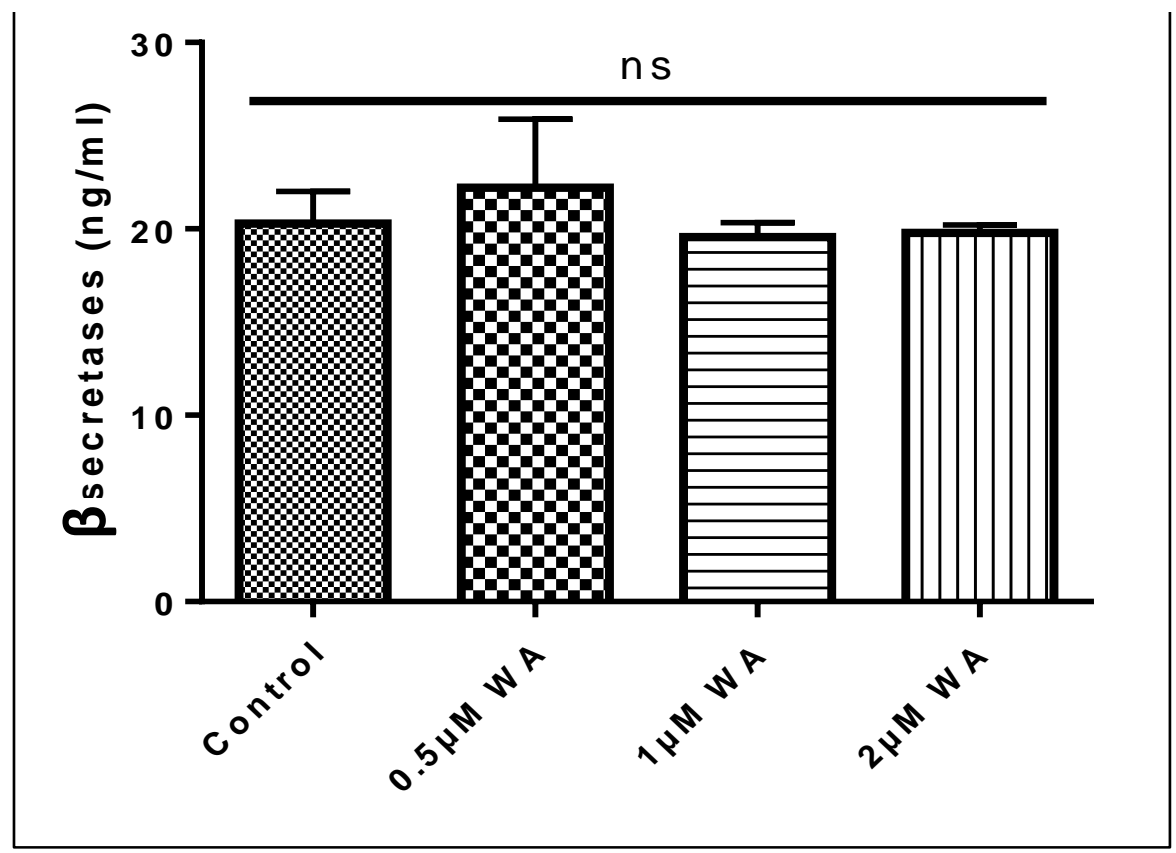

Figure 2.17 WA effect on the Beta secretases

No significant difference was observed in the levels of $\beta$ secretases in WA treated cells when compared to untreated control.

\section{$\underline{2.4 \text { Discussion }}$}

Neurological disorders are the biggest concern globally. Out of $\sim 36$ million HIV positive people about $30-60 \%$ exhibit neurological disorders including dementia and AD like pathology. In AD or AD like neurological disorders, the pathogenesis is mainly due to the abnormal accumulation of extracellular $A \beta$ in the CNS and is a major factor contributing towards neurodegeneration [73]. The introduction of HAART gives a longer life span, giving a major opportunity for developing age related disorders in these recovering patients [163-165]. The currently available drugs against $A \beta$ aggregation, for example, Memantine (N-methyl-D-aspartic acid (NMDA) receptor antagonist), helps in repair of damaged neurons [166], but does not aid in overall cure for neurological issues. Another 
drug which is very well studied for its anti-inflammatory, antioxidant and neuroprotective properties is Cucurmin/Curcuminoid, obtained from the roots of a plant Curcuma longa [167]. It has been reported that Curcuma may have potential role in AD treatment by targeting $A \beta$ aggregates and associated toxicity in the neuronal cells [168, 169]. Unfortunately, Curcuma is weakly stable and easily hydrolyzed, and gets photodegraded and even oxidized. This makes it very challenging and leads to its minimal bioavailability in the CNS [170].

Currently, there is no direct cure available for AD or AD-like neurodegenerative symptoms. Since plant based products are non-toxic and easily available, we planned to study the plant derived drug for this project. Therefore, here we have focused on a drug compound $W A$, and studied its role as a neuroprotective agent against $A \beta$ induced neuronal toxicity. Our in-vitro studies showed for the first time that a small sized active moiety of Withania root extract, WA decreases secreted $A \beta 40$, in the SHAPP cells without causing cytotoxicity. With the help of the microscopy studies, we were able to demonstrate the protective role of WA in the SHAPP cells, which showed healthy growth in the presence of WA. We observed that WA treatment reduced dendritic beading and cytoplasmic vacuoles in the SH-APP cells, conferring towards protective role of WA. Our observations on the role of WA, coincides with other studies, utilizing whole root extracts or parent compound $\mathrm{ASH}$, which show that $W$. somnifera whole root extract treatment promotes neuronal health by inducing dendrite formation in-vitro $[\underline{171}, \underline{172}]$. Moreover, our lab, in the previous studies, has also demonstrated the role of $A S H$ towards decreasing $A \beta$ in the neuronal cells in-vitro. ASH showed the reduction of $A \beta$ in treated cells significantly when compared to untreated controls, suggesting anti-amyloid role of $\mathrm{ASH}$. Nevertheless, even though $\mathrm{ASH}$ is capable of reducing the secreted $\mathrm{A} \beta$, the understanding of ASH's efficacy 
in the CNS across the BBB is minimum, as ASH molecule is big in size, and it is highly unlikely for it to cross the BBB to reach the brain. Therefore, the systematic delivery of the drug into the CNS and increasing bioavailability becomes a pressing issue. This urged a need to find potent smaller molecular weight molecules with similar properties. Systematic chromatographic studies show the various components, upon breakdown of ASH molecule. This gave us an opportunity to study small molecule WA and assess its ability as a neuroprotectant to target the $A \beta$ levels.

In addition to studying the role of WA in decreasing $A \beta$, we wanted to target Tat protein and drug of abuse, coc, induced increase in secreted $A \beta$ levels. Deposition of the $A \beta$ plaques in the CNS is one the major phenomenon in ageing HIV patients. Neurotoxic protein Tat is present in the brain even after ART administration and its interaction with $A \beta$ results in further increased levels of $A \beta$ [173-175]. Our study demonstrates the effect of WA on induced $A \beta$ production upon the exposure of HIV-1 Tat. Therefore, we analyzed the effect of HIV-1 Tat protein on the A $\beta$ secretion in SHAPP neuronal cells and found significantly increased $A \beta$ production in- vitro. Our results are in agreement with other studies, which have reported the role of Tat protein in increased neuronal $A \beta$ secretion [176-178]. The mechanism through which Tat increases $A \beta$ toxicity, is still not well understood, but some studies propose that Tat may have a direct interaction with the $A \beta$ fibrils, resulting in induced aggregation of monomers, towards plaques [145, 179]. This hypothesis is supported by the Immunocytochemistry studies, which show dense accumulation of $A \beta$, in the cell medium exposed to HIV-1Tat $(50 \mathrm{ng} / \mathrm{ml})$. The human $A \beta 40$ ELISA studies, demonstrated increased concentration of $A \beta 40$ in Tat treated samples. This lead us to a conclusion that Tat is extremely neurotoxic with an ability to interact with $A \beta$, increasing the overall toxicity, and contributing to increased aggravation of $A \beta$. 
Furthermore, drugs of abuse as cocaine is known to aggravate the toxic effects induced by HIV and associated products. Amongst the most abused drugs PLWH, coc abuse has been one of the major contributors towards the increased severity of neurocognitive disorders in the patients [180-182]. Additionally, the percentage of drug abusers in the HIV positive population and aging population is very high. Drug abuse/addiction and HIV/AIDS are linked since the beginning of the HIV/AIDS epidemic. People who inject drugs accounted for about $6 \%$ of HIV diagnoses in 2015 (CDC, 2018). Even though the association of $\operatorname{coc}$ is shown with the exaggeration in HIV neuropathogenesis, the underlying mechanisms remain unclear. We elucidate the mechanism, in this study, for the first time, we observed the increased levels of $A \beta$ production by coc. We observed in the Immunocytochemistry experiments that coc affects neuronal morphology and communications, and aggregation of A $\beta$ in the SHAPP cells, in-vitro. This verifies the toxic effect of coc on the neuronal cells, which contribute in the increased accumulation of the amyloids. Coc alone and in combination with HIV-1 Tat is highly neurotoxic. These results coincide with various in-vivo studies done by other research groups which show that the peritoneal injection of coc in rats stimulates hyperphosphorylation of tau and neurofilament in cortex, hippocampus and caudato-putamen regions of brain, indirectly contributing to the $A \beta$ toxicity [183]. These observations indicate that coc addiction may be associated with neurofibrillary degeneration. Therefore, here we report that coc in addition to HIV-1 Tat increases $A \beta$ secretion in vitro. To target the Tat and coc induced $A \beta$ secretion, we have proposed a potent bi-functional molecule WA that may act as a neuro-protectant against $A \beta$ neurotoxicity. Our findings suggest that HIV-1 Tat and coc introduce cellular toxicity and cause neuronal dysfunctions by increasing amyloid secretion and modulating neuronal morphology and communications. Moreover, accumulation and deposition of $A \beta$ in the brain of HIV patients (active infection or latent infection) drive the pathogenic 
cascades of neurological disorders, contributing towards aging or associated dementias [74]. Therefore, we have proposed that $A \beta$ deposition is induced by the presence of Tat and Cocaine and WA is potent in reducing the secreted $A \beta$ and induced neurotoxicity. Our study provides new opportunities for exploring the pathophysiology and targeting the neurological disorders. Targeting $A \beta$ secretion will have a translational significance in the treatment of HIV coc abusers and other neurological disorders like AD.

Further, in order to understand the mechanism of action of WA, we wanted to study the effect of WA, if any, on the enzymes involved in the $A \beta$ induced disease pathway. In our in-vitro studies, upon performing the ELISA studies with the cell supernatants, we did not observe any changes in the $\beta$ and $y$ secretases levels. This could be due to low amounts of these enzymes produced by the transfected SHAPP cells, which went undetected by the ELISA, in-vitro. In order to have a better understanding, we are currently performing in-silico studies to observe the structural interactions between $\beta$ and $\gamma$ secretases and WA if any, and we plan to do in-vivo studies to understand the effect of WA on these enzymes. 
CHAPTER 3: WA AND CRID3 AGAINST A $\beta$ INDUCED INFLAMMATION

\section{$\underline{3.1 \text { Introduction }}$}

$A D$ is caused by the buildup of intracellular neurofibrillary tangles made of tau protein and extracellular amyloid- $\beta$ plaques [184]. As discussed in Chapter 1, APP is cleaved by $\beta$ and $\gamma$ secretases and produces $A \beta$ peptides that locate and aggregate into the extracellular space. The formation of hydrophobic $A \beta$ plaques cause neuronal damage by contributing to cell death and affecting the synapses between neurons in cortical and limbic regions of the brain. There are various aspects which add to the severity of AD pathogenesis, which can be collected under wide constellation of medical risk factors comprising of cardiovascular diseases, heart failure, hypertension and high cholesterol [185-190], psychiatric risk factors like depression and anxiety [191], head injury [192, 193], stroke [194, 195], environmental factors or lifestyle factors like smoking or alcohol consumption [196-198] and pharmacological factors [199, 200]. Out of these contributing factors, inflammation is a potent threat towards $A D$ pathogenesis. $A D$ associated Inflammation and alterations in inflammatory markers (interleukins, cytokines), worsen the disease progression [201], correlating with all the associated neuropathological issues like neuronal degeneration, neuroinflammation, microglial activation, dysfunctions in BBB morphology and function, and finally cognitive deterioration and decline.

Therefore, it becomes important to understand the causes and the mechanism of inflammation with respect to $A D$ pathogenesis. Inflammation recruits more cellular elements to deteriorate the disease progression. There have been studies focusing on the inflammation aspects in $A D$, and several mechanisms have been proposed on the role of inflammation leading towards AD [202-204]. 
One of the main factors in inflammation is microglial infiltration, microglia play an important role against pathogenic protein aggregates, because they normally engulf $A \beta$ fibrils and produce the pro-inflammatory cytokines and chemokines in response to a protection mechanism. However, prominent microglial dysfunctions and associated imbalance between cytokines production and $A \beta$ clearance might be an additive factor towards $A D$ pathogenesis. The imbalance starts with the abnormal concentration of $A \beta$, moreover, the neuritic plaques are extracellular residues made of highly insoluble fibrillary $A \beta$ core containing protein fragments of about 39-42 amino acids. The aggregation of these plaques contribute to neurotoxicity, and boost immune response resulting in attracting microglial cells, reactive astrocytes, and dystrophic neurites fabricated by degenerating neuronal processes [205]. The aggregated plaques trigger series of cellular events, which prompt host immune response. High plaque density reflects heavy $A \beta$ accumulation, and this accumulation in contribution with resident cells migration, stimulates acute and chronic inflammatory responses inducing reactive oxygen species (ROS), nitric oxide (NO), and pro-inflammatory cytokines (Tumor necrosis factor- $\alpha$, Interleukin-1 $\beta$ and Interleukin -6 ), which lead to toxicity and consequent neuronal death [206-209] and aggregation of further more $A \beta$. Overall, there is a relationship between neuroinflammation caused by $A \beta$ plaques and neurodegeneration in the CNS.

Furthermore, the process of production of the pro inflammatory cytokine IL-1 $\beta$ is an extremely controlled process and is sensitive to several contributing factors. Primarily, it starts with the processing of Pro-IL-1 $\beta$, which is a biologically inactive form, to produce the active IL-1 $\beta$. This processing of Pro-IL-1 $1 \beta$ to active IL-1 $\beta$ is regulated by caspase- 1 enzyme. This enzyme requires processing and activation to be converted to an active enzyme, to perform its function, and its activation is mediated by high molecular weight 
protein complexes called as inflammasomes [210]. This happens via interaction of the Nod-like receptor protein (NLRP3) with the adapter molecule apoptosis-associated specklike protein to initiate inflammasomes production. Upon interaction, inflammasomes lead to caspase activation [211]. The NLRP3 are able to sense a distinct range of pathogens, insoluble factors, cellular stress and endogenous moieties like insoluble fibrils [212]. They are not directly activated but can sense intermediate processes or intermittent species like ROS [213]. NLRP3 protein expression levels are also a limiting step in inflammasome activation. IL-1 increasing in AD patients could be linked to $A \beta$ formation [214]. IL-1 is produced by microglial cells surrounding the neuritic plaques and contribute to initiate dystrophic neurite formation in $A \beta$ deposits $[\underline{215}, \underline{216}]$. Additionally, elevated IL-1 promotes an increase in P38 mitogen-activated protein (p38-MAP) kinases activity, leading to Tau hyperphosphorylation [217, 218].

Considering the significance of inflammation as a potent factor towards disease progression, therefore, in this project we studied the efficacy and role of WA and CRID3 (Cytokine Release Inhibitory Drug also termed as CP-424,174 and CP-412,245) against $A \beta$ induced inflammation. In literature, CRID3 have been studied to target ASC oligomerization in the NLRP3 inflammasomes [219]. Therefore, one of the main hypothesis of this chapter is that CRID3 acts as a neuroprotectant and may be able to target IL-1 $\beta$. Targeting IL-1 $\beta$ is extremely important, as IL-1 $\beta$ reduction and blockade decreases $A \beta$, associated Tau phosphorylation leading to decrease in neurotoxicity, and promote neurogenesis. We wanted to study the role of CRID3 as an anti-caspase and anti-IL-1 $\beta$ drug. We demonstrated the function of CRID3 as inhibitors of IL-1 $\beta$ production. 
Additionally, we have discussed the role of WA in Chapter 2, for decreasing A $\beta 40$, but in this chapter, we wanted to focus on the role of WA against inflammation. We wanted to analyze the role of WA against nuclear factor kappa-light-chain-enhancer of activated B cells (NF-kB) to understand the mechanism of WA action. NF-kB is a protein complex that has a role in controlling DNA transcription, cytokine production and cell survival. It has been established that the $A \beta$ aggregates activate microglia via TLRs and RAGE receptors. These receptors activate NF-KB, which induce the ROS production and the expression of inflammatory cytokines (IL-1, IL-6, TNF). These inflammatory factors additionally stimulate the astrocytes, amplifying the pro-inflammatory signals, leading to neurotoxicity [220].

In response to $A \beta$ induced microglial infiltration, in addition to increased inflammation and excess production of inflammatory cytokines, the NF-KB pathway is activated. The role of NF-KB in the expression of pro-inflammatory genes including cytokines, chemokines, and adhesion molecules has been studied [221]. Activated NF-KB is found predominantly in neurons and glial cells surrounding $A \beta$ plaques which induce an up-regulation of NF-KB activity and its translocation to the nucleus. NF-KB exerts effects on almost all cell types, playing an important function in inflammatory responses, therefore in this project, we have analyzed the effect of WA against NF-KB mediated inflammatory response. Our hypothesis is that WA may have a role in inhibiting the NF-KB mediated inflammatory response by inhibiting NF-kB expression during neurological disorders (Figure 3.1). 


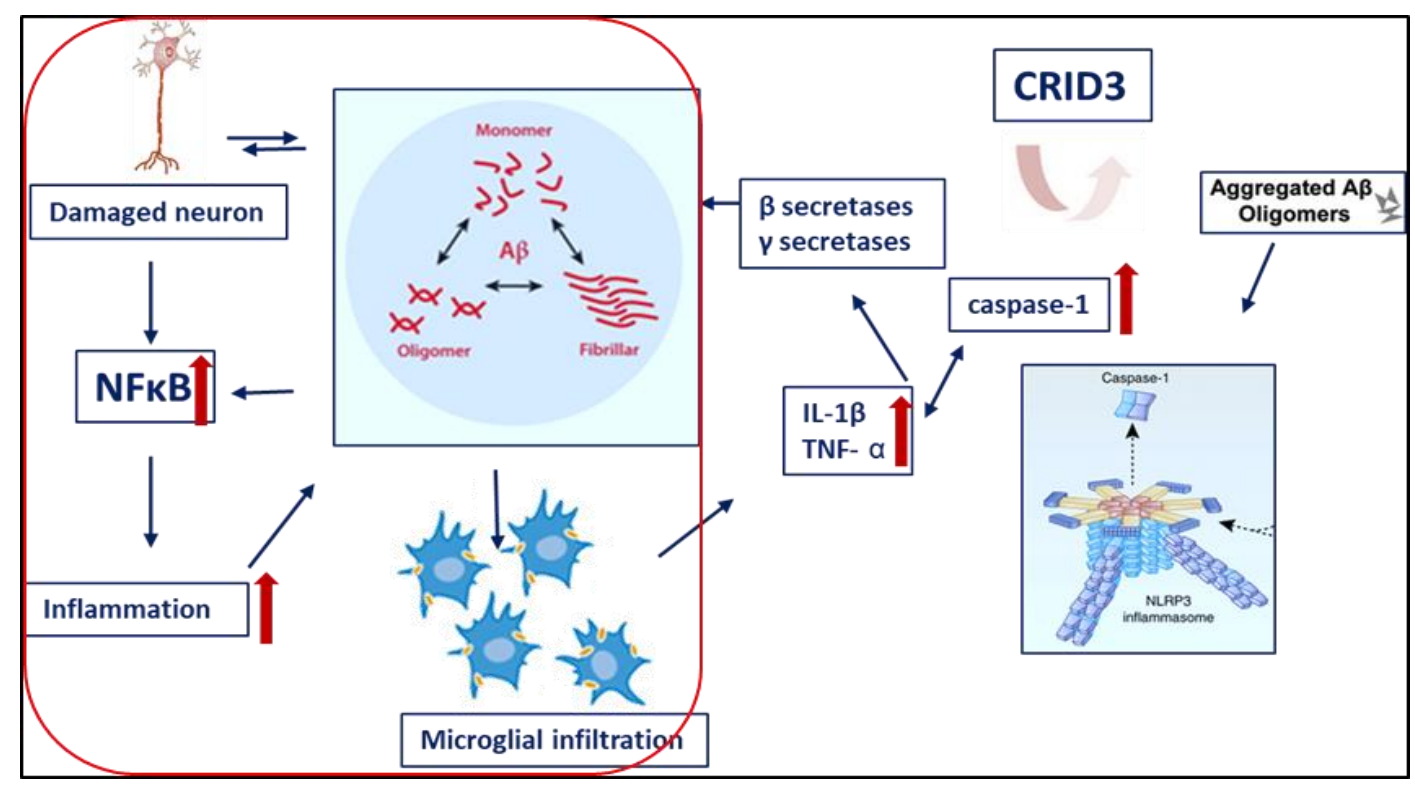

Figure 3.1 Hypothesis of WA and CRID3 against inflammation

The increased aggregation of $A \beta$ leads to series of events, which contribute in Inflammation and NF-KB plays an important role in AB associated inflammation which can be targeted by WA. Additionally, according to our hypothesis, CRID3 may reduce Caspase -1 enzyme activity, therefore may decrease active IL-1 $\beta$ levels during inflammation, therefore acting as anti-inflammatory drug

In addition to analyzing the role of WA and CRIDS individually, we have also explored the importance of developing a combination drug cocktail with the aid of nanotechnology, which can target inflammation caused by $A \beta$ in the brain. Here, we have designed a liposomal nanoformulation of CRID3 and analyzed its characteristics and efficacy to cross the BBB. We have also combined both the drugs into one liposome, and characterized the nanoformulation. Our future studies are designed to evaluate the functional efficacy of these developed combination liposomes. 


\subsection{Methodology}

\subsubsection{Cells, Chemicals and reagents}

SHAPP were received as a gift from Dr. Jonathan Geiger, University of North Dakota. WA was commercially purchased from Sigma Aldrich (Cat \# W4394 SIGMA), CRID3 was purchased commercially from Sigma Aldrich (Catalog no. PZ0280).

\subsubsection{Cell culture}

The cell type used in this study are SHAPP cells and were cultured in complete DMEM (explained in Methodology section of Chapter 2). We performed mixed cultures where the SHAPP cells were co-cultured with CHME5 microglial cell lines. CHEM5 also require DMEM for their growth, therefore, it was easier for us to culture these cells together.

\subsubsection{Cellular Toxicity assay}

CHME5 and SH-APP cells were grown separately in 96 well plates (20,000 cells/well) and incubated for $24 \mathrm{hrs}$. Cells were treated with different concentrations of WA $(0.5-2 \mu \mathrm{M})$, CRID3 (25nM-100nM) and MTM (0.1-1 $\mu \mathrm{M})$ and after 24hrs of incubation, the MTT cell viability assay was carried out. Wells were given with the media change with one $100 \mu \mathrm{l}$ medium and $10 \mu \mathrm{l}$ MTT (100 mg MTT / 20 ml PBS) was added for each well and incubated at $37^{\circ} \mathrm{C}$ for $2-3$ hours. After that, one volume $(110 \mu \mathrm{l})$ of stop solution was added and incubated with mild shaking for 3 hours. The optical density of the solubilized formazan was determined spectrophotometrically measuring the absorbance at $550 \mathrm{~nm}$. The optical density of formazan in each well is directly proportional to the cell viability and utilized for calculations. 


\subsubsection{Treatment with CRID3 and estimation of Caspase-1 and IL-1 $\beta$ levels}

SH-APP cells and CHME5 were co-cultured (2:1 ratio) in 6 well plates for $48 \mathrm{hrs}$, and treated with optimized concentration of CRID3 and incubated for further 48hrs. Cell supernatant was discarded and cells were trypsinized and centrifuged at $1500 \mathrm{rpm}$ for 5 mins, the cell pellets were collected in 1.5ml Eppendorf. Protein and RNA was isolated from these cells using the standard protocol from RNA and protein isolation kits. Protein samples were used to measure Caspase- 1 and IL-1 $\beta$ levels by the western blot assay using anti-Caspase-1 primary antibody (Thermo Fischer, Catalogue number PA1-37232) and anti-mature IL-1 $\beta$ antibody (Abcam) respectively.

\subsubsection{Estimation of NF-kB mediated neuroinflammatory genes on WA treatment} SH-APP cells and CHME5 were co-cultured (2:1 ratio) in 6 well plates for 48hrs, and treated with optimized concentration of WA and incubated for further 48hrs. Cells pellet was collected after trypsinization and centrifugation, Cells were lysed by Lysis buffer and RNA was isolated using the Qiagen Mini Easy RNA isolation kit using the protocol illustrated by the kit. $1 \mu \mathrm{g}$ of RNA was used for the first strand cDNA synthesis using SABiosciences's RT2 First Strand Kit (Cat \# 330401) as per supplier's protocol. Genomic DNA elimination step was performed before performing reverse transcription. $\mathrm{RT}^{2}$ Profiler $^{\text {TM }}$ PCR Array Human NF-kB Signaling Pathway kit (Qiagen Cat \# PAHS-025ZA12) was used to measure 84 key genes related to NF-KB mediated signal transduction. The array included genes that encode members of the Rel, NF-kB, and IkB families, NF$\mathrm{KB}$ responsive genes, extracellular ligands and receptors that activate the pathway, and kinases and transcription factors that propagate the signal. 


\subsubsection{Estimation of inflammasome mediated genes expression on CRID3 treatment}

From CRID3 treated cells, RNA was collected using the standard protocol. One microgram of RNA was used for the first strand cDNA synthesis using SABiosciences's RT2 First Strand Kit (Cat \# 330401) as per supplier's protocol. Genomic DNA elimination step was performed before going for reverse transcription. The Human Inflammasomes $\mathrm{RT}^{2}$ Profiler PCR Array (Qiagen Cat \# PAHS-097Z) was used to estimate the expression of 84 key genes involved in the function of inflammasomes, protein complexes involved in innate immunity, as well as general NOD-like receptor (NLR) signaling.

\subsection{Results}

\subsubsection{CRID3 and WA do not cause cellular toxicity}

Before utilizing CRID3 to test its role against inflammation, we tested the toxicity of CRID3 on the SHAPP and CHME5 cell types that we used for the experiments. We treated CHME5 microglial cell lines and SH-APP human neuroblastoma cells for 24 hours and observed that the range of CRID3 $(5 \mathrm{nM}-2 \mu \mathrm{M})$ was not toxic to both the cell types and did not effect the cell viability compared to untreated controls (Figure 3.2). Based on MTT assay, we selected the minimal concentration of CRID3 to be utilized for further experiments. 

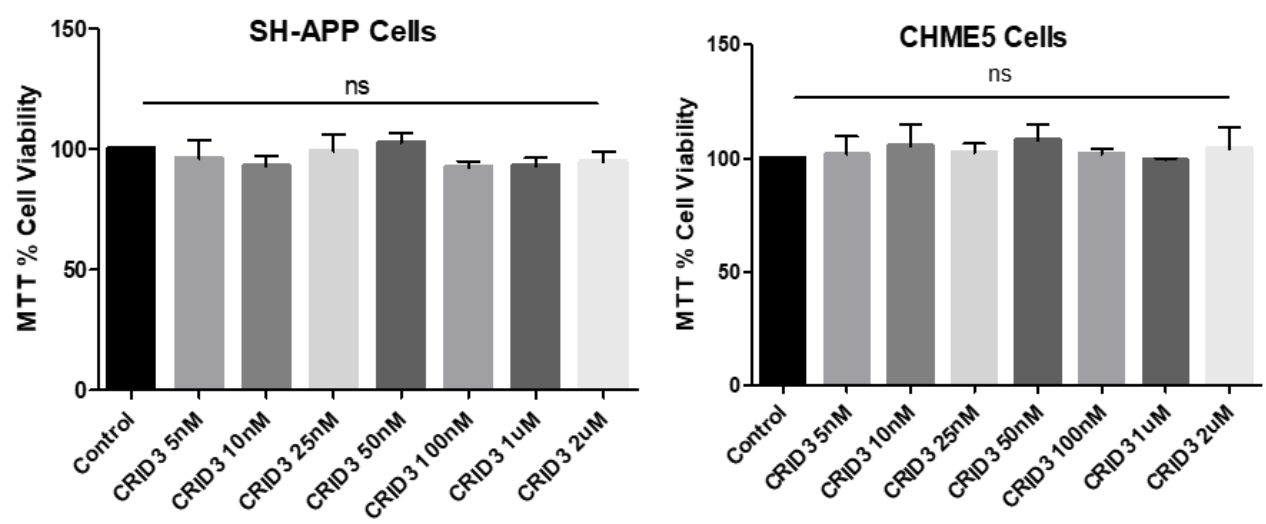

Figure 3.2 MTT Cell Viability Assay

SH-APP cells and microglial cells were exposed to different concentrations of WA (50nM-1 $1 \mu \mathrm{M})$ and CRID3 $(5 \mathrm{nM}-2 \mu \mathrm{M})$. After 24 hours of incubation, cell viability was analyzed by MTT assay. We observed no cytotoxicity with WA and CRID3 up to $2 \mu \mathrm{M}$.

\subsubsection{WA inhibits inflammatory regulated genes associated with NF-k $\beta$ pathway}

To analyze the efficacy of WA on the inflammatory factors, SHAPP cells and CHME5 Microglial cell lines were co-cultured for $24 \mathrm{hrs}$ and incubated with WA. After $48 \mathrm{hrs}$ of treatment, cells were analyzed for the expression of NF-KB mediated inflammatory response mediators using the Human NF-kB Signaling Pathway PCR Array. We observed that WA inhibited the expression of NF-KB (Nuclear Factor Kappa $\beta$ Subunit 2) and RELA transcription factors which plays a major role in the expression of inflammatory chemokines and cytokines. We also observed the IKBKB and IKBKG up-regulation (depletion of these protein activates the NF-KB) and JUN and STAT gene down-regulation. Furthermore, we observed down-regulation of IL-1 $1 \beta$, which plays a major role in the NFKB mediated neuroinflammation (Table 3.1). 
Table 3.1 WA inhibits NF-k $\beta$ mediated inflammatory response related genes expression array

Human microglia and SHAPP cells were co-cultured (microglia: neuron ratio of $1: 2)$ and exposed to WA $(2 \mu \mathrm{M})$. After $48 \mathrm{hrs}$ of incubation, we observed significant down-regulation of transcription factors, pro-inflammatory cytokines and apoptosis inducing gene expression in the culture indicate the NF-Kb mediated anti-inflammatory activity of WA.

\begin{tabular}{|c|c|}
\hline Genes & $\begin{array}{c}\text { WA } \\
\text { (Fold Change) }\end{array}$ \\
\hline IL1B & -4.0558 \\
\hline IL-10 & 4.69 \\
\hline NFKB1 & 1.6818 \\
\hline NFKB2 & -1.2834 \\
\hline REL & 1.3755 \\
\hline RELA & -1.021 \\
\hline RELB & 1.2746 \\
\hline BCL3 & -1.2226 \\
\hline CHUK & -1.1975 \\
\hline IKBKB & 1.4743 \\
\hline IKBKG & 2.1287 \\
\hline NFKBIA & 1.014 \\
\hline NFKBIE & 1.3287 \\
\hline JUN & -1.181 \\
\hline STAT1 & -1.0281 \\
\hline
\end{tabular}

\subsubsection{CRID3 inhibits inflammatory regulated genes associated with NLRP3 pathway}

The co-culture was exposed to $100 \mathrm{nM}$ concentration of CRID3 (100nM concentration chosen based on cell viability studies). After $48 \mathrm{hrs}$ of incubation, cells were harvested, RNA was isolated and Human Inflammatory Cytokines \& Receptors PCR Array was performed. Results showed that CRID3 significantly down-regulated various chemokines 
and cytokines like Complement component 5 (C5), Chemokine (C-C motif) ligand 20 (CCL20), Chemokine (C-C motif) ligand 26 (CCL26), Chemokine (C-C motif) ligand 5 (CCL5) and receptors like Chemokine receptor 6 (CCR6), Interleukin 8 receptor, beta also known as CXCR2. It also down-regulated IL-1 inflammatory cytokine gene expression and Interleukin 33 (IL-33) pro-inflammatory cytokines Additionally, Interferon Gamma (IFNG) gene expressing IFNy was observed to be up-regulated (Table 3.2). This indicates towards the anti-inflammatory property of CRID3 as it inhibits IL1- $\beta$. According to the result from this array, we have further analyzed the effect of CRID3 on the IL1- $\beta$ and its processor Caspase-1 activities. 


\section{Table 3.2: CRID3 inhibits inflammatory gene expression}

Human microglia and SHAPP were co-cultured (microglia: neuron ratio of $1: 2)$ and treated with 100nM CRID3. After 48hrs of incubation, we observed significant down-regulation of chemokines and pro-inflammatory cytokines gene expression, and cytokine receptors, in the cells, indicating towards the anti-inflammatory activity of CRID3 and indicating towards CRID3 as important molecule against Beta amyloid associated inflammation.

\begin{tabular}{|c|c|}
\hline Genes & $\begin{array}{c}\text { CRID3 } \\
\text { (Fold Change) }\end{array}$ \\
\hline IL1B & -1.05 \\
\hline C5 & -1.14 \\
\hline CCL20 & -1.78 \\
\hline CCL26 & -1.17 \\
\hline CCL5 & -1.33 \\
\hline CCR6 & -1.44 \\
\hline CXCR2 & -1.21 \\
\hline IFNG & 1.51 \\
\hline IL33 & -1.42 \\
\hline
\end{tabular}

\subsubsection{CRID3 inhibits Caspase-1 and IL-1 $1 \beta$ protein expression}

Caspase-1 (interleukin-1 converting enzyme) and consequently released mature IL-1 $\beta$ production plays a major role in the neuroinflammatory response in the AD patients. In this study, we have used various concentrations of CRID3 (25nM-100nM) to measure its efficacy in inhibiting caspase- 1 activation and IL-1 $1 \beta$ production in SHAPP cells co-cultured with CHME5 microglia cell line (microglia: neuron ratio of 1:2). This co-culture was 
incubated for 48hrs in the presence of the CRID3 (100nM). After 48 hours, the cells were harvested and the protein was isolated by lysing the cells in RIPA buffer. The isolated protein was estimated for its concentration by Bradford protein estimation method. Using western blot analysis, we analyzed the expression of both Caspase-1 and mature IL-1 $\beta$ protein expression. Data showed significant down-regulation of both Caspase-1 and mature IL-1 $\beta$ protein levels in the samples treated by $100 \mathrm{nM}$ CRID3 (Figure $3.3 \mathrm{~A}, \mathrm{C}$ ) compared to other concentrations of CRID3 and untreated control. The decreased levels of Caspase- 1 and mature IL- $1 \beta$ indicates towards potential therapeutic role of CRID3 in the prevention of neuronal inflammation due to the accumulation of $A \beta$ in $A D$ patients. We also quantified the protein using image $\mathrm{J}$ software which shows significant decrease of Caspase-1 and mature IL-1 $\beta$ in $100 \mathrm{Nm}$ CRID3 treated samples compared to untreated controls (Figure 3.3 B, D). 


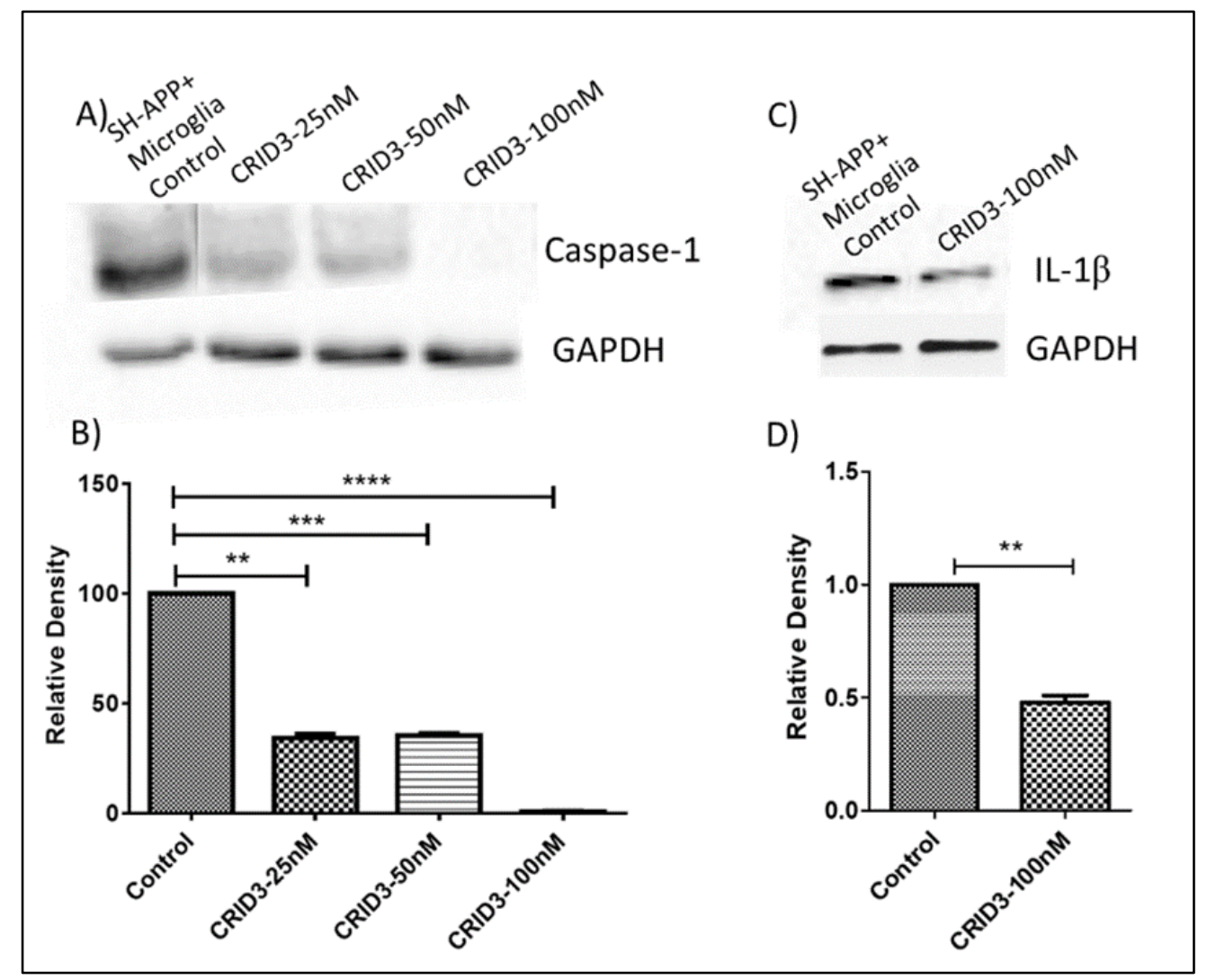

Figure 3.3 CRID3 inhibits Caspase-1 and IL-1 $\beta$ protein expression SH-APP cells were co-cultured with microglia (microglia: neuron ratio of $1: 2)$ in the presence of CRID3 (25-100nM) for $48 \mathrm{~h}$. Cells were harvested and cell pellet was used in western blot for Caspase-1 (A) and IL-1 $\beta$ (C) analysis. Relative protein expression was calculated using ImageJ and observed significant inhibition of Caspase-1 (B) and IL-1 $\beta$ (D) protein expression at 100nM CRID3 concentration compared to the untreated control cells $\left(^{* *}, p \leq 0.01 ;{ }^{* * *}, p \leq 0.001 ;{ }^{* * * *}, p \leq 0.0001\right)$.

\subsubsection{Liposomal Nanoformulation of CRID3}

Having studied the importance of CRID3 against IL-1 $\beta$ via inhibiting Caspase-1, henceforth inhibiting amyloid associated inflammation. It became utmost important for us to increase the bioavailability of hydrophilic CRID3 in the CNS, across the BBB. Therefore, in this project we have attempted to design a nanoformulation for the effective 
transmigration of CRID3 across the in-vitro 3D blood brain barrier, and to analyze the drugbinding efficacy to the liposomal nanoformulation. CRID3 before being used for the liposomal nanoformulation was studied for it toxicity on the SHAPP cells. We found that CRID3 was not toxic to the cells. We designed the liposomes by the combination of Egg PC, Cholesterol and Chloroform as an organic solvent. Upon utilizing the dehydration method of liposomal preparation and loading an initial concentration of $100 \mu \mathrm{g} / \mathrm{ml}$ CRID3 in the lipid cocktail, we were able to extrude the single bi-layered liposomes with the loaded CRID3. According to the DLS studies, the developed CRID3 liposomes were characterized as $480+/-30 \mathrm{~nm}$ in size and had $78.94 \%$ drug loading efficacy (Figure 3.4).

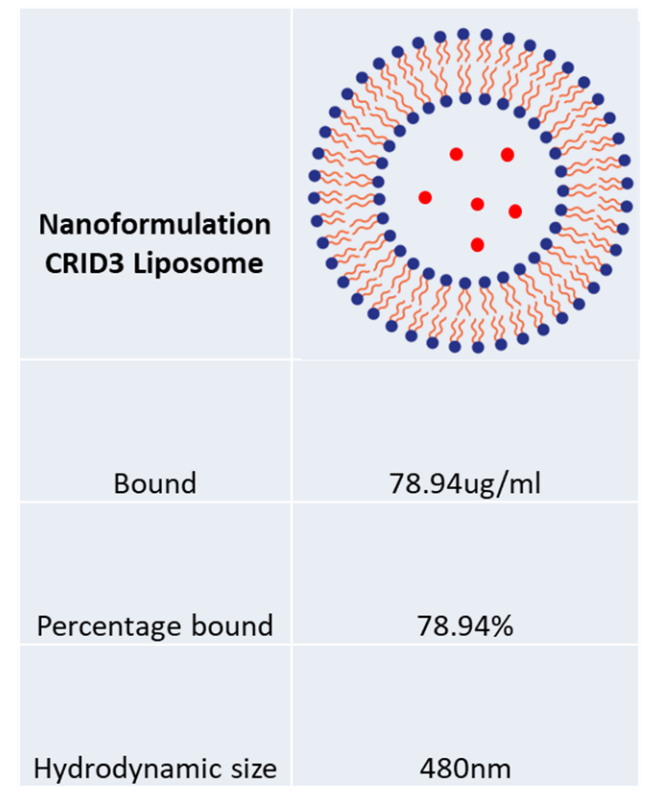

Figure 3.4 Designed CRID3 Liposomal Nanoformulation (CRID3-LNF) and its characterization.

The designed and produced liposome had a hydrodynamic size of 480nm and effectively carried approximately $78.94 \%$ of hydrophilic CRID3 drug in its hydrophilic core, and it was non-toxic to the cell culture. 


\subsubsection{Liposomal CRID3 and its transmigration across BBB}

The transmigration of CRID3-LNF across the BBB caused no effect on TEER values indicating towards no deteriorating effect on BBB resistance (Figure 3.5) and the transmigration efficiency was approximately 50\% (Figure 3.6). CRID3 being a hydrophilic drug, is trapped in the hydrophilic core of the bi-layered liposomal nanoformulations (Figure 3.4). The successful transmigration of CRID3-Liposomal Nanoformulation across the BBB make it important for the drug delivery strategies. CRID3 has showed to be decreasing IL-1 $\beta$ via decreasing Caspase-1 activity. In addition, aiding it transmigration across BBB to increase its bioavailability into the CNS is a promising step towards battling amyloid induced inflammation.

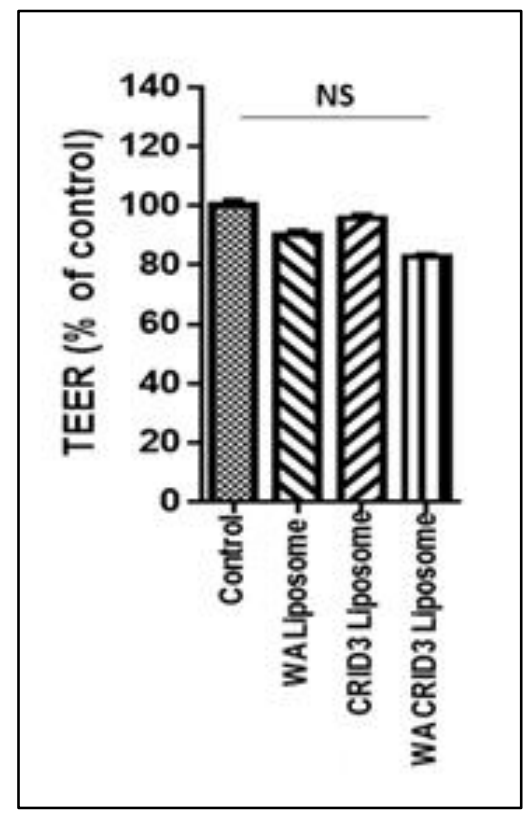

Figure 3.5 In-vitro effect of NF on the integrity of 3D BBB model

3D in-vitro BBB model was established and NFs were added on top of the BBB and incubated for $24 \mathrm{~h}$ at $37^{\circ} \mathrm{C}$ to facilitate the transmigration across the BBB and the internalization of the liposome. Integrity of BBB was determined by measuring the Trans endothelial electrical resistance of the membrane by using TEER measuring gauge. 


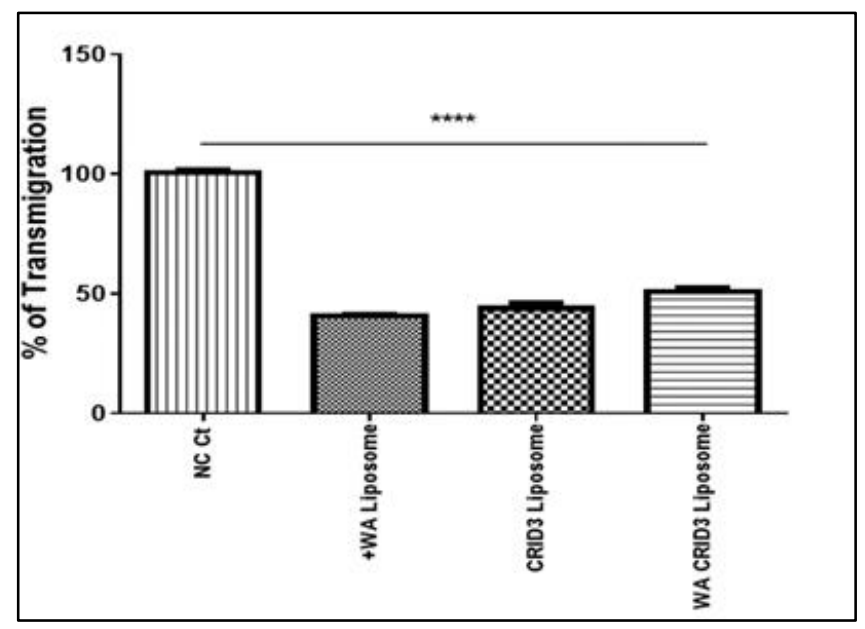

Figure 3.6 In-vitro NF transmigration across the 3D BBB model

3D in-vitro BBB model was established and NFs, free drugs, and HIV-1 Tat were added on top of the BBB under magnetic field exposure for $24 \mathrm{~h}$ to facilitate the transmigration across the BBB and the internalization of the liposome. The percentage of NF transmigration across the BBB was measured using the FITC-labelled liposome.

Hence, the developed CRID3-LNF were stable and crossed the BBB with 50\% transmigration efficiency without causing any deteriorating effect on the resistance of the BBB membrane. The nanoformulation has acceptable hydrodynamic size and aided efficient transmigration of CRID3 across the BBB. Liposomes were made of egg-PC (phosphatidylcholine) therefore, they were biocompatible and non-toxic to the barrier cells.

\section{$\underline{3.4 \text { Discussion }}$}

Amyloid plaques, built of the $A \beta$ protein, are the hallmark neuropathological features in the full-blown $A D$ brain $[134, \underline{222}, \underline{223}]$. $A \beta$ in addition to causing neuronal toxicity also plays a very crucial role in activating innate immune system and attracting microglial infiltration leading to induction of inflammation $[224,225]$. $A \beta$ is considered as an inducer of microglial activation and neuroinflammation, and poses as an underlying factor in the 
development of AD [204]. Microglia have dual role in AD pathogenesis, they are involved in $A D$ pathogenesis as when coming in contact with $A \beta$ peptides, they release inflammatory mediators such as inflammatory cytokines, complement components, chemokines, and free radicals like ROS which subsidize further $A \beta$ production and its aggregation into big and insoluble plaques [226]. There is continuous dependent relationship between $A \beta$, microglial infiltration, production of inflammatory mediators and induction of inflammation (Figure 3.7).

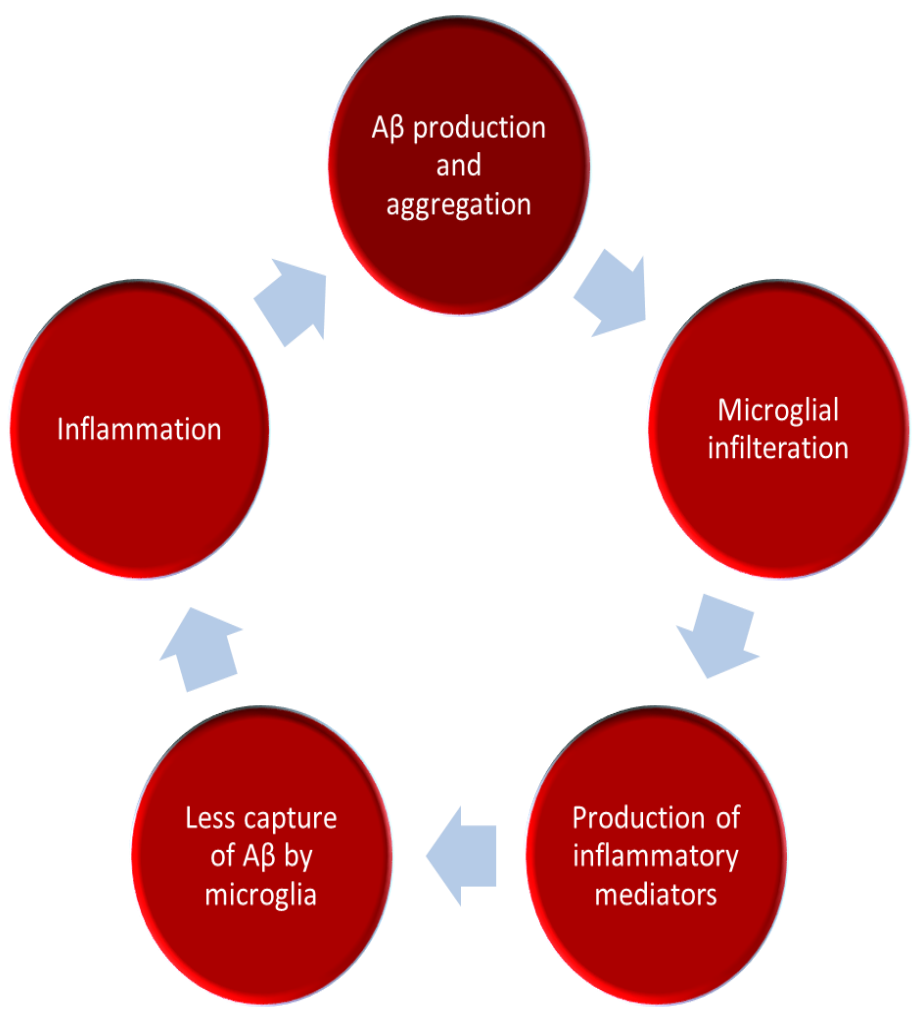

Figure 3.7 The interdependent relationship between between $A \beta$, Microglial infiltration, production of inflammatory mediators and finally inflammation. 
This interdependent pathogenesis pathway requires combination drugs cocktail to target inflammation and $A \beta$ induced toxicity. $A \beta$ aggregation activates microglia via TLRs and RAGE receptors and in turn these receptors activate NF-k B. Increased activity of NF-K B induce ROS production and the expression of inflammatory cytokines [227]. Therefore, in this chapter, we have studied the drugs WA and CRID3 for their roles against $A B$ and $A \beta$ induced inflammation.

The nuclear factor NF-KB pathway is a pro-inflammatory signaling pathway, inducing expression of pro-inflammatory genes including cytokines, chemokines, and adhesion molecules [221]. The NF-kB pathway includes Nuclear factor-KB (NF-kB)/Rel proteins include NF-kB2 (p52/p100), NF-kB1 (p50/p105), c-Rel, RelA/p65, and RelB proteins. These proteins together play role as dimeric transcription factors regulating innate and adaptive immunity, inflammation and stress response associated gene expressions. NF$\mathrm{KB} /$ Rel proteins are bound and inhibited by nuclear factor of kappa light polypeptide gene enhancer in B-cells inhibitor (IKB) proteins. But with the introduction of pro-inflammatory cytokines, Lipopolysaccharides, fibrillar antigens etc. there is an activation of an I kappa B kinase (IKK) complex (IKKß/IKKa), which then phosphorylates IKB proteins leading to IKB ubiquitination and proteasomal degradation, and releasing the NF-KB/Rel complexes. Active NF-KB/Rel complexes undergo post-translational modifications and translocate to the nucleus where, with the help of other factors like Stat, induce target gene expression. In the alternative (or non-canonical) NF-KB pathway, NF-kB2(p100)/RelB complexes are inactive in the cytoplasm and activate IKKa that phosphorylate NF-KB2 p100 leading to its ubiquitination and proteasomal processing to NF-KB2 p52 which then translocates to the nucleus and induces target gene expression [228-230]. 
Since a wide array of diseases, including cancer, are rooted in inflammation, there is a surge in interest in understanding the mechanistic regulation of inflammatory responses. NF-KB activation is widely implicated in inflammatory diseases like rheumatoid arthritis, Atherosclerosis, Asthma, Multiple sclerosis, chronic obstructive pulmonary disease etc. [231] and therefore development of anti-inflammatory drugs targeting NF-KB are currently in focus [232]. $A \beta$ aggregation may indirectly result in elevated NF-kB. We here hypothesized that WA may be able to inhibit NF-Kb associated inflammation. With the NF$\mathrm{kB}$ signaling array studies, we observed that WA inhibits inflammatory regulated genes associated with NF-kB pathway. The NF-kB assay experiment demonstrated that WA was able to inhibit the expression of NF-kB2 (Nuclear Factor Kappa B Subunit 2). NF-kB2 encodes a subunit of the transcription factor complex NF-kB. The NF-kB complex is expressed in numerous cell types and functions as a central activator of genes involved in inflammation and immune function [233]. Several studies have shown the regulation of the NF-KB2 (p100) in response to the inflammatory cytokines and have shown that IL-1 $\beta$, induces p100 expression at mRNA and protein level [234]. Therefore, if WA is downregulating NF-KB2 that means it may be equally capable of indirectly down-regulating IL1ß. NF-kB is composed of NF-KB1 or NF-KB2 bound to either REL, RELA, or RELB. The amplest form of NF-kB is NF-KB1 complexed with the product of this gene, RELA (also known as p65), which is a REL-associated protein participating in NF-KB heterodimer formation, aiding nuclear translocation and its activation [235]. We also observed the down-regulation of RELA transcription factor which plays a major role in the expression of inflammatory chemokines and cytokines. NF-kB activity at inflammation sites activates the canonical pathway and RelA containing complexes. Therefore, down-regulation of RelA indirectly down-regulates NF-kB. In addition to these, we have also observed the IKBKB (Inhibitor of nuclear factor kappa-B kinase subunit beta) and IKBKG (NF-kappa-B 
essential modulator) up-regulation. IKBKB phosphorylates components of the NF-kB signaling pathway including IKBKG, NF-kB subunits RELA and NF-KB1, as well as IKKrelated kinases TBK1 and IKBKE. This phosphorylation may help in preventing the overproduction of inflammatory mediators, highlighting the role of WA against NF-KB regulation, proving its anti-NF-kB potency. Furthermore, we have observed up-regulation of NF-KBIA (NF-kappa-B inhibitor alpha) which inhibits the activity of dimeric NF-KB /REL complexes by trapping REL dimers in the cytoplasm [236] and NF-KBIE or IKBE (NFkappa-B inhibitor epsilon) which Inhibits NF-kB by complexing with and containing it in the cytoplasm [237]. Additionally, most importantly we observed that WA was able to

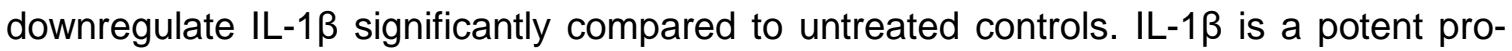
inflammatory cytokine and its production includes CASP1 activation, pro-IL-1 $1 \beta$ processing and secretion of the active IL-1 $\beta$ leading to inflammasome assembly [238]. We showed that WA treatment modulates the level of multiple factors in co-cultured cells, associated with NF-KB down-regulation. Therefore, a reduction in IL1- $\beta$ may be associated with the inhibition of NF-KB by WA treatment. Studying NF-KB is important, as some Insilico studies have showed the promoter regions of $\mathrm{IL}-1 \beta$ to be possessing NF-KB transcription factor sites. Therefore NF-kB down-regulation may be connected to IL-1 $\beta$ down-regulation [239] All these factors assert the important role of WA against inflammation and WA inhibits NF-kB mediated neuro-inflammation.

In addition to WA, we have also deciphered the role of CRID3. Microglial cells produce inflammatory cytokines in response to various stimuli like insoluble toxic protein $A \beta$ [240]. The inflammation array studies demonstrate that increased inflammation increases the inflammatory cytokines/chemokines levels, but CRID3 reduced A induced inflammatory mediators and cytokine production, including IL-1 $\beta$ and Caspase- 1 . 
However, we could not observe its effect on intracellular ROS in SHAPPs, but it could be a restriction of in-vitro experiments. We will try to look at ROS production in our ongoing in-vivo studies. Upon analyzing the array data, we found that CRID3 down-regulated IL$1 \beta$ significantly compared to untreated controls. To assess the potency of CRID3 against IL-1 $\beta$, we performed protein quantification by utilizing western blot technique and observed the down-regulation of IL1- $\beta$ and Caspase-1 protein expression upon treatment with CRID3. The cytokine cascade in inflammatory processes is complex that involves stimulants, glial cells, neuronal cells and immune system. IL-1 $\beta$ is one of the important factors, it is a pro-inflammatory cytokine that has been associated in inflammation and pain [241]. The IL-1 cytokine family is primarily associated with acute and chronic inflammation [242]. To better understand the inflammatory responses it is important to understand the role of inflammasome complex proteins like IL-1 $1 \beta$ which has main role in inflammasome activation [243, 244]. We saw the reduction in IL-1 $\beta$, in WA and CRID3 treated samples that could be due to reduced pro IL-1 $\beta$ and reduced active caspase-1. Although IL-1 $\beta$ secretion and reduction might depend on various factors [245], but according to our study it may be due to NF-KB reduction and anti-inflammasome role of WA and CRID3.

The cytosolic segment of IL-1 receptor contains the Toll-IL-1-receptor domain that is common in Toll-like receptors, which respond to external factors like viruses or microbial factors. IL-1 $\beta$ is studied to be a therapeutic target against inflammatory diseases and its decrease is important for disease reduction [246]. Further, the assay demonstrated that CRID3 down-regulates various chemokines and cytokines like Complement component 5 (C5), Chemokine (C-C motif) ligand 20 (CCL20) also called as Macrophage Inflammatory Protein-3 (MIP3A), Chemokine (C-C motif) ligand 26 (CCL26) also called as Eotaxin-3 or 
Macrophage inflammatory protein 4-alpha (MIP-4), Chemokine (C-C motif) ligand 5 (CCL5) also known as RANTES (regulated on activation, normal T cell expressed and secreted) which have been studied for their chemotactic properties. It also down-regulated the receptors like CCR6 and CXCR2. Our studies highlight that CRID3 has role against IL-1 $\beta$ in lower concentrations and causes no cytotoxicity.

Therefore, in conclusion it is important to say that the chronic deposition of $A \beta$ stimulates the persistent activation of microglia and results in increased IL-1 $1 \beta$ levels (IL-1 $\beta$ requires caspase- 1 for activation). Caspase-1 activity is controlled by inflammasomes. The NLRP3 inflammasome can sense inflammatory crystals and aggregated proteins, including $A \beta$. Therefore, inhibition of NF-kB mediated inflammatory response and inhibition of NLRP3 mediated inflammatory response is important. We introduce cytokine release inhibitory drugs or CRIDs as inhibitors of secretion of IL-1 1 . Therefore, in this study, use of CRID3 will be useful for the prevention of NLRP3 mediated inflammatory response induced by $A \beta$. These amyloid peptides in turn lead to increased oxidative stress and increased inflammation [247]. Microglia release pro-inflammatory cytokines and (TNFa) that upregulate $\beta$ and $y$ secretases and lead to increases in $A \beta 42$, therefore a cocktail of antiamyloid and anti-inflammatory drug and its delivery to the CNS was the most important aim of this project.

Having studied the importance of CRID3 against $A \beta$ associated inflammation, the next important and pressing issue is the bioavailability of the drug CRID3 across the BBB into the CNS. For this, we devised the strategy of the development of liposomal nanoformulation of CRID3 separately and as a combined cocktail formulation with WA. Liposomes are one amongst the common, efficient, biocompatible and well-studied 
nanocarriers for the drug delivery with increased efficacy and improved targeted delivery. Liposomes are exploited for their ability to stabilize drugs, increase cellular and tissue uptake, and refining and increasing the bioavailability and bio distribution of therapeutic drugs [248-251]. Based on these benefits of liposomes, we decided to design liposomes as a carrier for CRID3. The developed liposomes were loaded with CRID3, which being hydrophilic was packed in the hydrophilic core of the liposome. The drug was loaded in the liposome with great efficacy and did not contribute in increasing the hydrodynamic size of the liposomal complex. CRID3 loaded liposomal nanoformulation transmigrated across the cellular layer of the in-vitro BBB model which was measured by the transendothelial electrical resistance (TEER) measurement method [252]. Measuring TEER ensures that the developed nanoformulation is not toxic to the BBB and passes through the without effecting the permeability or the stability of the membrane. The designed liposome based on its efficacy can be of immense therapeutic importance against inflammation and toxicity caused by $A \beta$. Moreover, we have developed a nanoformulation with both WA and CRID3 to act as one formulation, multiple effects strategy. Incorporation of WA in the hydrophobic and CRID3 in the hydrophilic part of liposomes, will help us target $A \beta$ and inflammation associated with $A \beta$ with just one nanoformulation. This study is ongoing and is part of our future perspectives to evaluate its efficacy in-vivo. This strategy could be of immense therapeutic potential against $A \beta$ induced inflammation in $A D$ or $A D$ - like diseases. 
CHAPTER 4: LIPOSOMAL NANOFORMULATION OF WITHAFERIN A AS A PROMISING NANOCARRIER AGAINST AMYLOID BETA

\subsection{Introduction}

The ageing population is prone to age-related neurological disorders, including motor disorders, memory deficit, dementia and various other neurological issues [253, 254]. [255]. The most fatal and complex of neurological diseases is $A D$ or $A D$ like pathology [256] characterized by progressive loss of synapses and neurons, due to buildup of amyloid plaques, and neurofibrillary tangles, and associated inflammatory factors leading to excessive toxicity in the CNS [257]. The presence of cerebral amyloid deposition are confirmed by studies using positron emission tomographic (PET) brain scans with the aid of amyloid tracers, which also suggest that $A \beta$ may pose as one of the important target for AD detection and therapeutics [258]. This hypothesis has currently lead to a current surplus of drugs being investigated and developed against $A D$ or $A D$-like diseases targeting $A \beta$ as one of the potential biomarkers. The researchers are continuously looking for new options to treat $A D$ and associated dementia.

Drug developing field faces a major challenge, in the areas of methods development for increasing the bioavailability of the drugs in the CNS to the physiologically relevant levels. The shortcoming of the current drug strategies are the minimalistic availability of the drugs in the patient brain. The presence of semi-permeable BBB at the brain-blood junction makes it nearly impossible for the drug molecules to penetrate through and reach the brain [259-262]. Preliminary strategies to overcome the transportation issues involved passive diffusion of small lipophilic molecules but it was not acceptable, as this strategy could not include the vast majority of potential therapeutic molecules. Nevertheless, next approach 
was to develop water soluble small molecular size drugs, to facilitate their transport across BBB via paracellular diffusion pathway, but these molecules failed to penetrate past the tight junctions of endothelial cell layers [263]. Therefore, since the drugs do not reach the brain in the efficacious concentration, it has become urgent for the employment of the emerging technologies to aid in effective delivery of anti-amyloid drugs across the BBB effectively into the CNS.

Currently, nanotechnology has gained tremendous interest over the past several decades in the field of drug delivery. Nanotechnology employs various targeted nanosystems as drug carriers, which are highly capable in increasing therapeutic efficacies as they have effective role in regulating the bio distribution, circulation time, stability and solubility of the drugs. All these factors are immensely important for a successful therapeutic advancement [264-266]. Smart nano-systems are being currently designed for effective delivery of the drugs to the unapproachable body parts like brain and spleen. These nanoparticles are in range from 50-500 nano-meter and can be tagged by fluorescent factors to help with the visualization purposes upon the administration of the Nanoparticles in the targeted areas. They can be targeted to the respective areas where it is required the most and the drug binding stability and on-demand release of the drugs contribute as positive add-ons to the emerging nanotechnological advances.

There are various nanoformulations, which are being developed and tested for their efficacy in drug delivery. Out of them, liposomal nanoformulations have been investigated as effective drug delivery agents. Liposomes have the ability to co-encapsulate the drug of interest (hydrophobic or hydrophilic) in addition to the contrast agents. These contrast agents can help in tracking the drug and its distribution towards the target area [267-269]. 
The BBB is a sensitive biological dynamic barrier made up of tight junctions (TJs) between endothelial cells lining the blood vessels, end-feet of astrocytes, and a basement membrane. Role of pericytes is also important in increasing the stability of the membrane and the tight junctions. Together these cells regulate the CNS milieu and are essential to maintain the uncontaminated microenvironment of the brain [269-271]. The barrier is so tightly packed that it leaves immensely little chance for an alien molecule to pass through. The transport properties of the semi permeable BBB is limited to cells, water and ions, and is extremely selective to solutes, nutrients, therapeutic agents and drug carriers. TJs have a regulating effect, and reduce the ion and other hydrophilic solutes permeation by paracellular pathway, therefore contributing as the physical barrier [272]. Nonetheless, BBB also poses as a barrier against the delivery of the vital drugs, which are of immense importance to treat CNS associated diseases. Therefore, it requires serious efforts to devise nano-carriers for the improvement of drug delivery across the BBB to target and treat neuropathological conditions like dementia, AD, Parkinson's, and HIV associated dementia.

Amongst various strategies for increasing drug delivery including physical methods like focused ultrasound [273], chemical modifications like modifying a drug into a prodrug to enhance lipophilicity [274] and biological modifications like tagging drugs with macromolecules (proteins and peptides to aid BBB transmigration by endocytosis) [275], the strategy of nanoparticles based drug-delivery system is competent and more efficacious and promising [276].

With concern to efficacious hydrophobic drug delivery across the BBB, the most feasible, promising and targeted drug delivery system against $A D$ are Liposomes. Liposomes are 
biocompatible and flexible in nature, and have the potential to carry multiple therapeutic molecules across the BBB. In the early 1960s the development and therapeutic prospective of liposomes was studied 1961, but their importance as effective nanocarriers has been understood very recently in the field of neuroscience [277, 278]. Liposomes are bi-layered lipid entities which incorporate hydrophilic or lipophilic therapeutic agents, where hydrophilic drugs are entrapped in the aqueous core, and lipophilic compounds are contained in the hydrophobic region of the lipid bilayer $[\underline{279}, \underline{280]}$.

There are various studies where liposomes are employed as the career for the drug delivery [281]. Liposomes demonstrate high efficiency for nose-to-brain transport, for a promising strategy for drug delivery in the brain $[\underline{282}, \underline{283}]$. The current study incorporates the design of modified targeted liposomes, which are efficient across BBB and can focus on the specific molecular targets in the CNS concerning AD (Figure 4.1).

Studies suggest that liposomes are able to penetrate in the cells [284] through, either with the aid of phospholipid bilayer of the liposomes on its own which might enable transportation or by taking benefit of the negative charge of the BBB. The lipids 1,2dipalmitoyl-sn-glycero-3-phosphocholine (DPPC), Phosphatidylcholines (PC) and cholesterol component of liposomes help enhance the cellular uptake [285]. Therefore, they serve as great research alternative approaches for drug delivery in case of CNS diseases. The approach in this chapter is based on liposomal delivery of the neuroprotectant WA, which is discussed in previous chapters, decreases $A \beta$ induced toxicity and inflammation. Our laboratory has previously studied the importance and mechanisms of nanoparticles and theranaustics in the field of drug delivery [264]. Here we have focused on liposomes, which have been widely researched for diverse medical 
applications including hydrophobic or hydrophilic drugs delivery, non- toxicity and easy release of the drugs at the target, allowing for immense potential in various biomedical applications.

In the present in-vitro study, we have focused on the drug binding properties of liposomal nanoformulation with WA, to aid its transmigration across the BBB and increase WA's bioavailability. This may be a promising strategy for AD, AD-like pathology and HIV associated dementia patients. Our approach started with the liposomal synthesis process employing multiple combinations of lipids versus cholesterol ratios and then the final developed drug loaded liposomal products were characterized for their properties. Special care was taken to investigate the subsequent effect of each condition on the morphology of the liposomes, and the best-suited dehydration time, hydration temperature and lipid ratios were confirmed. Developed liposomes were characterized and studied for their transmigration and efficacy across BBB. 

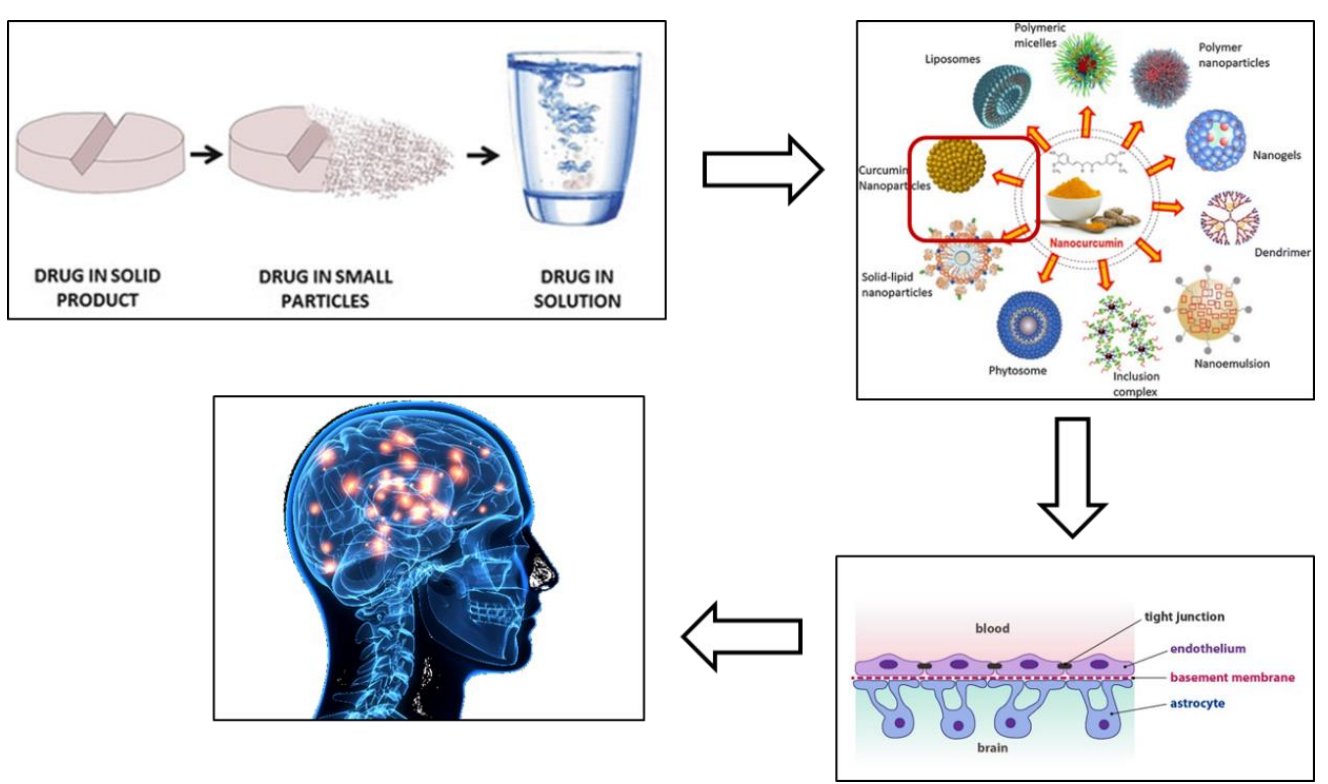

Figure 4.1 The flow-plan of the strategy to increase the bioavailability of the drugs in to the CNS across the BBB

\section{$\underline{4.2 \text { Materials and methods }}$}

\subsubsection{The 3D in-vitro BBB model}

Primary human brain microvascular endothelial cells (HBMESs), human astrocytes (HAs) and human pericytes (HPs) cells were cultivated as per the provider's recommendations (ScienCell Research Laboratories, CA, USA). The BBB model was established as described by Persidsky et al. [286]. In brief, the in-vitro BBB model was developed in a bicompartmental trans-well culture plate (product 3415; Corning Life Sciences, Mexico). The upper chamber of this plate is separated from the lower chamber by a $10 \mu \mathrm{m}$ thick polycarbonate membrane possessing $3.0 \mu \mathrm{m}$ pores. In a sterile, 24 -well cell culture plate with a pore density of $2 \times 10^{6}$ pores $/ \mathrm{cm}^{2}$ and a cell growth area of $0.33 \mathrm{~cm}^{2} .2 \times 10^{5} \mathrm{HBMEC}$ were grown and $1 \times 10^{5}$ each of $\mathrm{HA}$ and HP were grown to confluence on the upper 
chamber and underside of the lower chamber, respectively. The trans-wells were flooded with the combination media (ECM: $A M$ : $P M=2: 1: 1)$, the upper chamber can hold up to $600 \mu \mathrm{l}$ media, and lower chamber can hold $500 \mu \mathrm{l}$ of media. After the cells were adhered to the transwell inserts, these inserts were transferred to 24 wells plate. This setup was cultured at $37^{\circ} \mathrm{C}$ and the media was changed every 48 hours. The $3 \mathrm{D}$ in-vitro BBB was used for further experiments after 5 days. Intactness of the in-vitro 3D BBB model was determined by measuring the TEER, using Millicell ERS microelectrodes (Millipore) on the $5^{\text {th }}$ day after the initial seeding.

\subsubsection{BBB transmigration assay}

Transmigration study of drug-loaded WA liposomes was conducted on the 5th day of the BBB culture, when ideal integrity of the membrane was achieved, as established by TEER measurement experiment. To assess the effect of liposomal nanoformulation on the integrity of the in-vitro BBB model, transmigration assay was performed to measure paracellular transport of FITC-dextran [287]. 100 mg/mL FITC-dextran (Sigma-Aldrich, St Louis, MO, USA) was added to the upper chamber of the inserts and further incubated for 6 hours. Samples were collected from the bottom chamber after 6 hours, and relative fluorescence was measured at excitation wavelength $485 \mathrm{~nm}$ and emission wavelength 520 nm, using a Synergy HT multimode microplate reader (BioTek Instruments, Inc., Winooski, VT, USA) multimode microplate reader instrument. FITC-dextran transport was expressed as percentage FITC-dextran transported across the BBB into the lower compartment compared with negative (untreated) control. WA liposomal nanoformulation was added to the apical chamber and incubated at $37^{\circ} \mathrm{C}$ for 48 hours. Supernatant were collected from the lower chambers and percentage of transmigration was analyzed at different points, using an ammonium thiocyanate-based photometric assay [288]. 


\subsubsection{Synthesis of WA Liposomal Nanoformulation}

For the experiments, we used fluorescent and non-fluorescent liposomes. The liposomes were synthesized using the dehydration method. For the preparation of Multilamellar Liposomes (LMV), the seed solution was prepared by adding $80 \mu \mathrm{L}$ of egg-PC lipid, $20 \mu \mathrm{L}$ of Fluorescent egg-PC (100 $\mathrm{L}$ of egg-PC in case of non-fluorescent liposomes), $0.5 \mathrm{mg} / \mathrm{ml}$ of Cholesterol, and was made up to total volume of $200 \mu \mathrm{L}$ by dissolving the lipids in Chloroform, in a clean and dry glass tube. The solution was then mixed well for 1 minute and loaded in the vacuum Rotary evaporator system (Yamato RE-201 Rotary Evaporator, BM-200) for the evaporation of the organic solvent chloroform. The tube was dipped in the hot water bath, and water temperature was maintained at $40^{\circ} \mathrm{C}$. The setup was set at mild rotations, for facilitating proper mixing and dehydration of the chloroform. After 30 minutes of dehydration, the tube was checked for the dried up thin and translucent film at the base of the glass tube. $1 \mathrm{ml}$ of fresh PBS $(1 \mathrm{ml})$ was added to the glass tube. The solution was carefully vortexed for a couple of minutes until the film dissolved completely in the PBS and the mouth of the tube was sealed with parafilm to avoid contamination. The tube was kept in water bath for 1 hour with regular vortexing intervals at every 20 minutes. The temperature of the water bath was maintained at $40^{\circ} \mathrm{C}$. These LMVs were extruded into large unilamellar Vesicles (LUVs). Meanwhile the mini- extruder setup was placed on a hot plate (502-P, PMC Industries, Inc., San Diego, CA, USA) for approximately 30 mins. A thermometer was inserted into the well provided in the heating block, and was allowed to reach the temperature of $50^{\circ} \mathrm{C}$. After rehydrating the LMVs for 1 hour, and once the sample was fully hydrated, we loaded the sample into one of the gas-tight syringes and carefully place into one end of the Mini-Extruder. Second empty gas-tight syringe was placed into the other end of the Mini-Extruder set-up. The fully assembled extruder

apparatus was inserted into preheated extruder stand. The LMV sample was pushed 
across the $0.2 \mu \mathrm{m}$ membrane by gently pushing the plunger of the filled syringe until the lipid solution is completely transferred to the alternate syringe via porous membrane and the membrane filter. The step was repeated 5 times making 10 passes through membrane as more the passes though the membrane, the more homogenous the lipid solution becomes. The final extrusion filled up the alternate syringe, and the solution was collected from there into the Eppendorf $1.5 \mathrm{ml}$ tubes (Figure 4.2) [289-291]. The filtered solution was then ultra- centrifuged at $100,000 \mathrm{~g}$ for 1 hour at $4^{\circ} \mathrm{C}$. The supernatant was collected (unbound drug) and the pellet (Drug bound LUVs) was re-suspended in $1 \mathrm{~mL}$ of PBS. The drug binding was calculated indirectly by subtracting total drug from the unbound drug, measure by spectrometer.

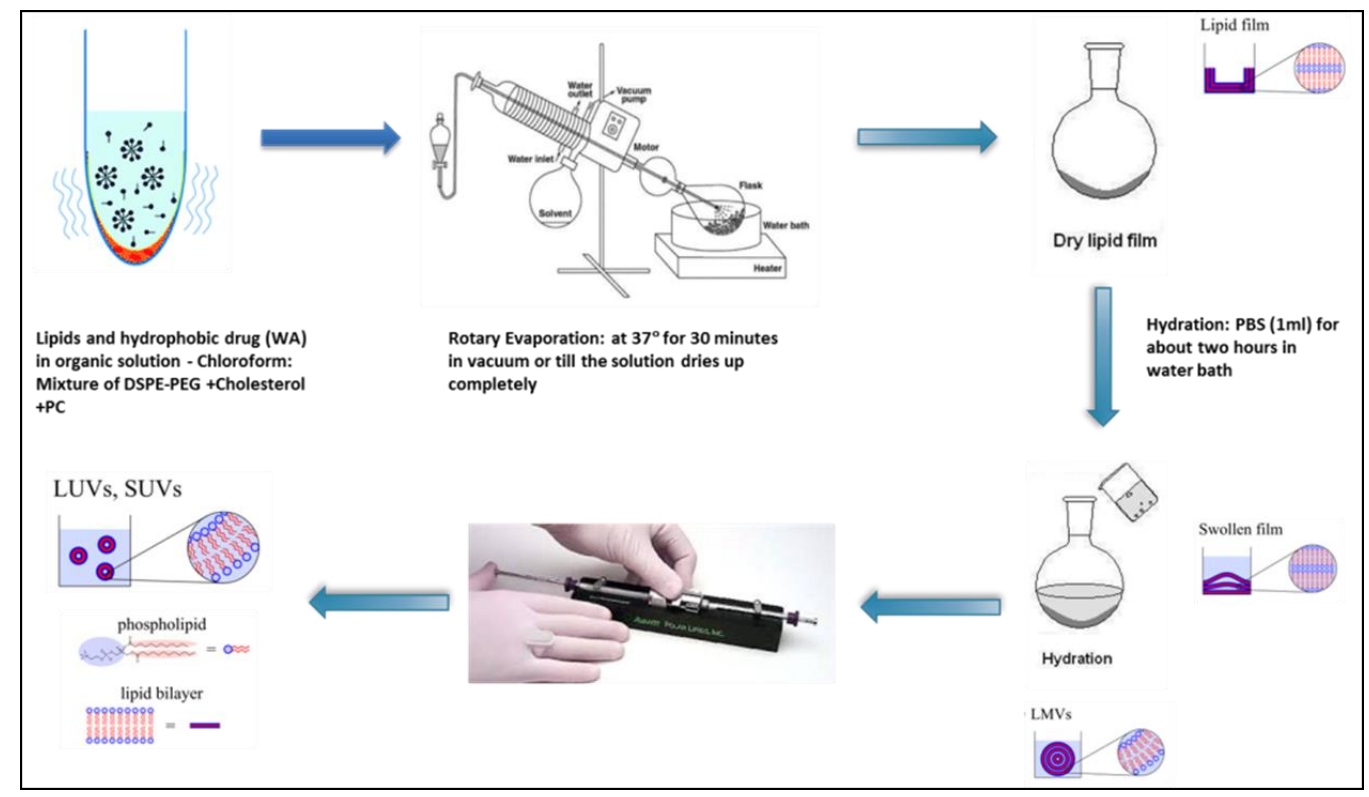

Figure 4.2 Preparation of Liposome Nano formulation Liposome was prepared by hydration method using variant ratios of lipids dispersed in organic solvent Chloroform 


\subsubsection{Characterization of liposomes size and bound drug calculation}

A zetasizer was used to calculate the average size of the developed liposomes. The drug bound liposomes from the pellet of the centrifuged liposomal samples were redispursed in PBS and measured for their size. The amount of unbound drug was predicted by analyzing the concentration of unbound drug in the supernatant at $230 \mathrm{~nm}$ and hence indirectly the amount of bound drug was calculated by subtracting unbound drug from the initial drug concentration. The drug binding was calculated in percentage by keeping initial concentration of the drug as $100 \%$. All these concentrations were calculated based on the standard curve, which was prepared using different concentration of WA read at $230 \mathrm{~nm}$ wavelength.

\subsubsection{Toxicity studies: Trypan Blue Cell viability test}

The cells were grown at the concentration of $0.1 \times 10^{6} / 2 \mathrm{ml}$ and were incubated for 48 hours at $37^{\circ} \mathrm{C}$. After 48 hours, the cells were trypsinized and collected. The cells were centrifuged and the pellet was re-suspended in $1 \mathrm{ml}$ of fresh media. $10 \mu \mathrm{l}$ of cells suspension was mixed with $1 \mathrm{X}$ Trypan blue dye in the ratio of $1: 1$ and was loaded onto the slides and read by viable cell counter. The control was considered as $100 \%$ and the viability of other samples were calculated with respect to control.

\subsubsection{AB40 ELISA with Liposomal NF}

Secreted $A \beta$ levels were measured using human $A \beta 40$ ELISA kit as per the manufacturer's protocol (Thermo Fisher Scientific, Catalog\# KHB3481). For secreted A $\beta$ measurements, the SH-APP cell supernatant were collected from the basal side of the BBB. Protease inhibitor was added to the supernatant to avoid protein degradation. The supernatant was utilized as samples for the AB40 ELISA as per the specific protocol provided with the kit. 


\subsubsection{WA Drug release kinetics from the Liposome by Dialysis membrane method}

WA Liposomes were prepared and were re-dispersed in final volume of $1 \mathrm{ml}$ PBS. $500 \mu \mathrm{l}$ of WA Liposomal solution was then loaded to the micro Float-A-Lyzer with the help of $1 \mathrm{ml}$ syringe. The micro Float-a-Lyzer (The Spectra/Por Micro Float-A-Lyzer) has an ultrapure Biotech Grade Cellulose Ester (CE) tubular membrane with a volume size of 400 - $500 \mu \mathrm{l}$ with the Molecular weight cut off range of $3.5-5 \mathrm{kD}$. The set up was placed in a glass flask of $200 \mathrm{ml}$ volume filled with $60 \mathrm{ml}$ of dissolution medium Phosphate buffer $(\mathrm{pH} 7.4)$. The speed of rotation for this setup was $2000 \mathrm{rpm}$ and the temperature was maintained at 25 $\pm 0.5^{\circ} \mathrm{C}$. Aliquot of $1 \mathrm{~mL}$ at each time points were withdrawn and replaced with $1 \mathrm{~mL}$ of phosphate buffer pH 7.4 (Time Points:0, 0.5,1,1.5,2,4,8,10,17, 18, 19, 23and 48 hours). The samples were analyzed by UV spectrophotometry analytical method at $230 \mathrm{~nm}$, and the drug released was calculated based on WA standard curve. The percent release was calculated accordingly.

\subsubsection{Data Analysis}

The experiments were repeated at least three times and the values obtained were averaged. All the results were expressed as mean \pm standard error of the mean. Statistical analysis of two groups was performed by Student's $t$-test, while more than two groups were analyzed using one-way ANOVA. Data analysis was performed with the Statistical Program, Graph Pad Prism software (La Jolla, CA, USA).

\subsection{Results}

\subsubsection{In-vitro 3D Blood Brain Barrier model}

The in-vitro 3-D Blood brain barrier was made with the primary human CNS cells; HBMEC, HA and PA. The intactness of the blood brain barrier was measured by measuring TEER 
values which were +/- 230 indicating towards, intactness of the BBB membrane. This intact BBB was used further for the treatment with the nanoformulations and for the transmigration experiments. Figure 4.3 is the representative diagram of the developed invitro 3D model of the BBB.

\subsubsection{Development and characterization of NF}

Schematic representation of the developed liposomes loaded with neuroprotectant drug WA is shown in Figure 4.4. The bi-layered liposomes were prepared and characterized for their size, toxicity and drug binding efficiency.

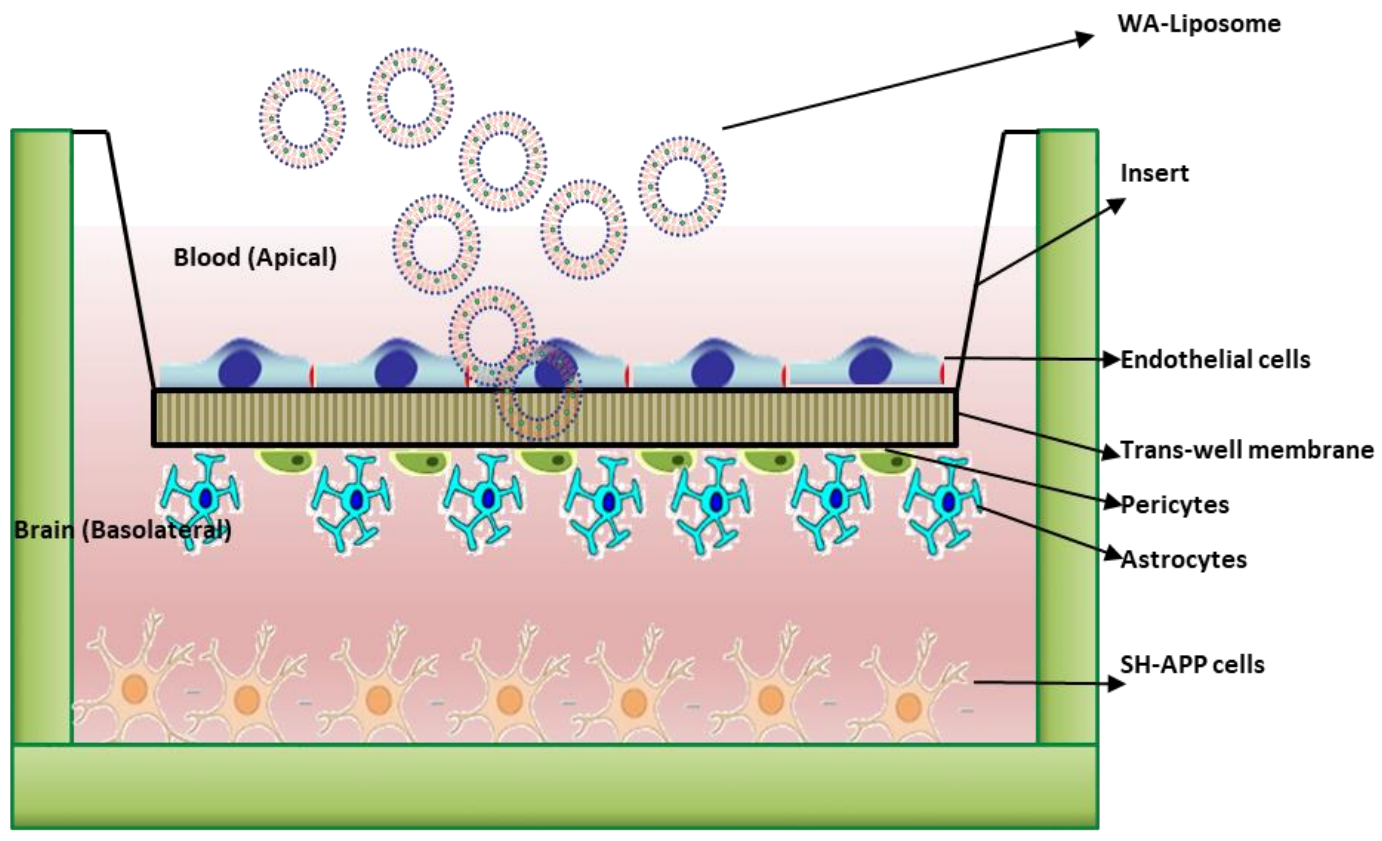

Figure 4.3 Developed in-vitro 3D BBB model demonstration

This figure demonstrates the strategy of designing the in-vitro 3D BBB model. The Endothelial cells are grown at the apical side and Astrocytes and Pericytes were grown at the basolateral side. The cells were allowed to grow and firmly attach to the membrane in the trans-well insert. The media used was a combination media of $A M$ : PM: ECM in a ratio of $1: 1: 1$. The BBB was allowed to grow at $37^{\circ} \mathrm{C}$ and $5 \% \mathrm{CO}_{2}$ and then the inserts with attached cells were transferred in the new wells with SH-APP growing at the base of these wells. The TEER was measured after 5 days, to assess the intactness of the BBB. 


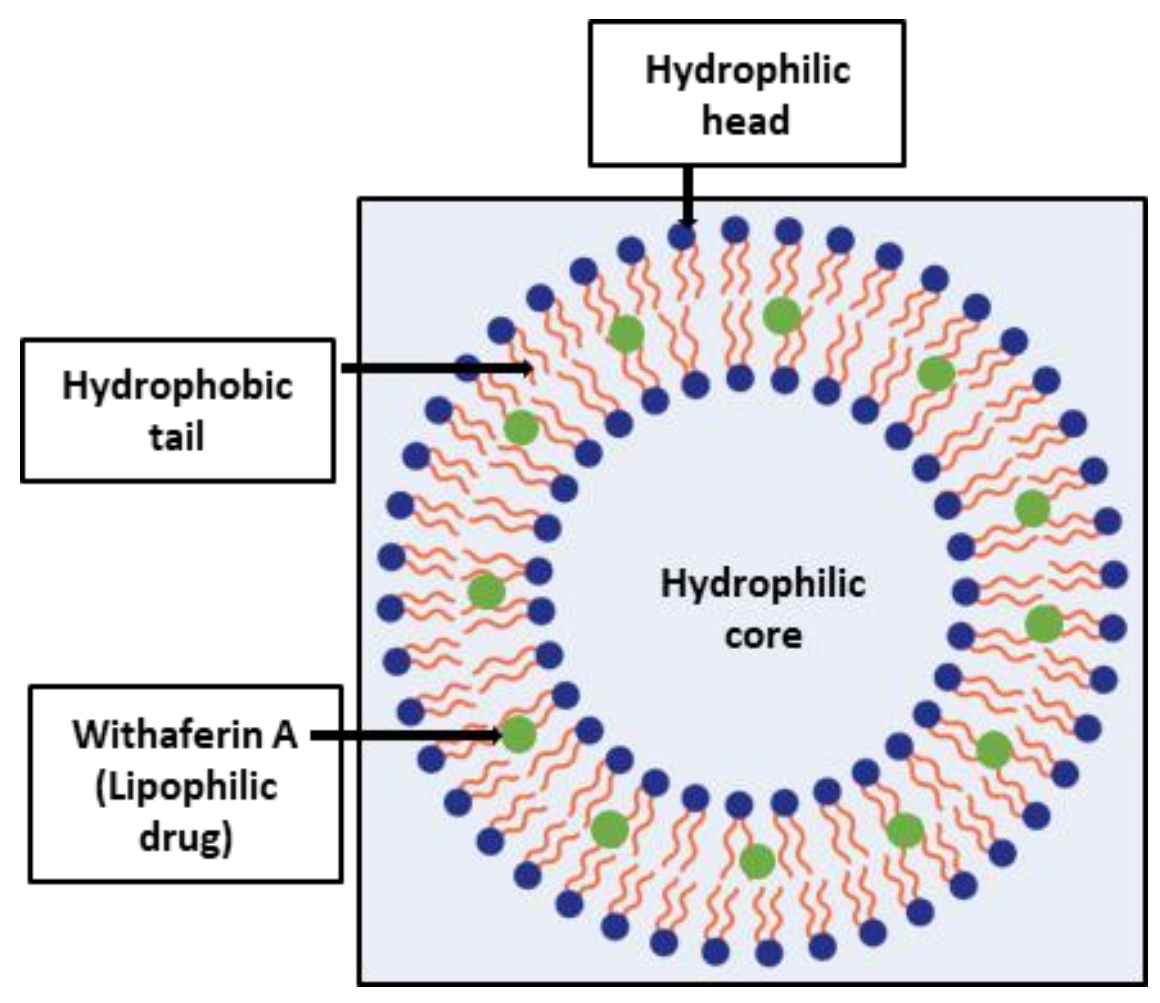

Figure 4.4 Schematic liposome structure

The figure demonstrating the phospholipid bilayer, consisting of hydrophilic head and hydrophobic tail. The entrapment of hydrophobic drug WA by the lipid bilayer is also demonstrated.

The developed WA-liposomal nanoformulation (WA-LNF) demonstrated the average size of approximately $400 \pm 50 \mathrm{~nm}$ diameter (as they were only bath sonicated and not probe sonicated), and they were uniform in size and shape as studied by Dynamic light scattering (DLS) analysis used for hydrodynamic size analysis, as shown in Table 4.1. The loading efficiency of liposomes were calculated indirectly by estimating unbound drug in the supernatant after ultracentrifugation of the prepared liposomal solution. We found that the developed liposomes had a bound drug concentration of $14.14 \mu \mathrm{g} / \mathrm{ml}$, which was approximately $28 \%$ drug binding efficacy when compared to the initial 50 $\mu \mathrm{g} / \mathrm{ml}$ loaded drug concentration while preparing the liposomes. To encapsulate the 
hydrophobic WA in the liposome, different solvent combinations (Chloroform or Chloroform to Ethanol in 90:10 or 50:50 ratios) were used for the synthesis of liposomes. Table 4.1 highlights the characterization of nanoformulations with respect to drug loading, bound percentage and hydrodynamic size of the liposome. For the final assembled liposome nanoformulation, we used Chloroform to Ethanol in 50:50 ratio as WA is easily soluble in Ethanol.

Table 4.1: Characterization of developed Liposomal Nanoformulation The developed WA Liposomal NF is stable, non-toxic and shows efficient drug binding and transmigration across the in-vitro 3D BBB trans-well model

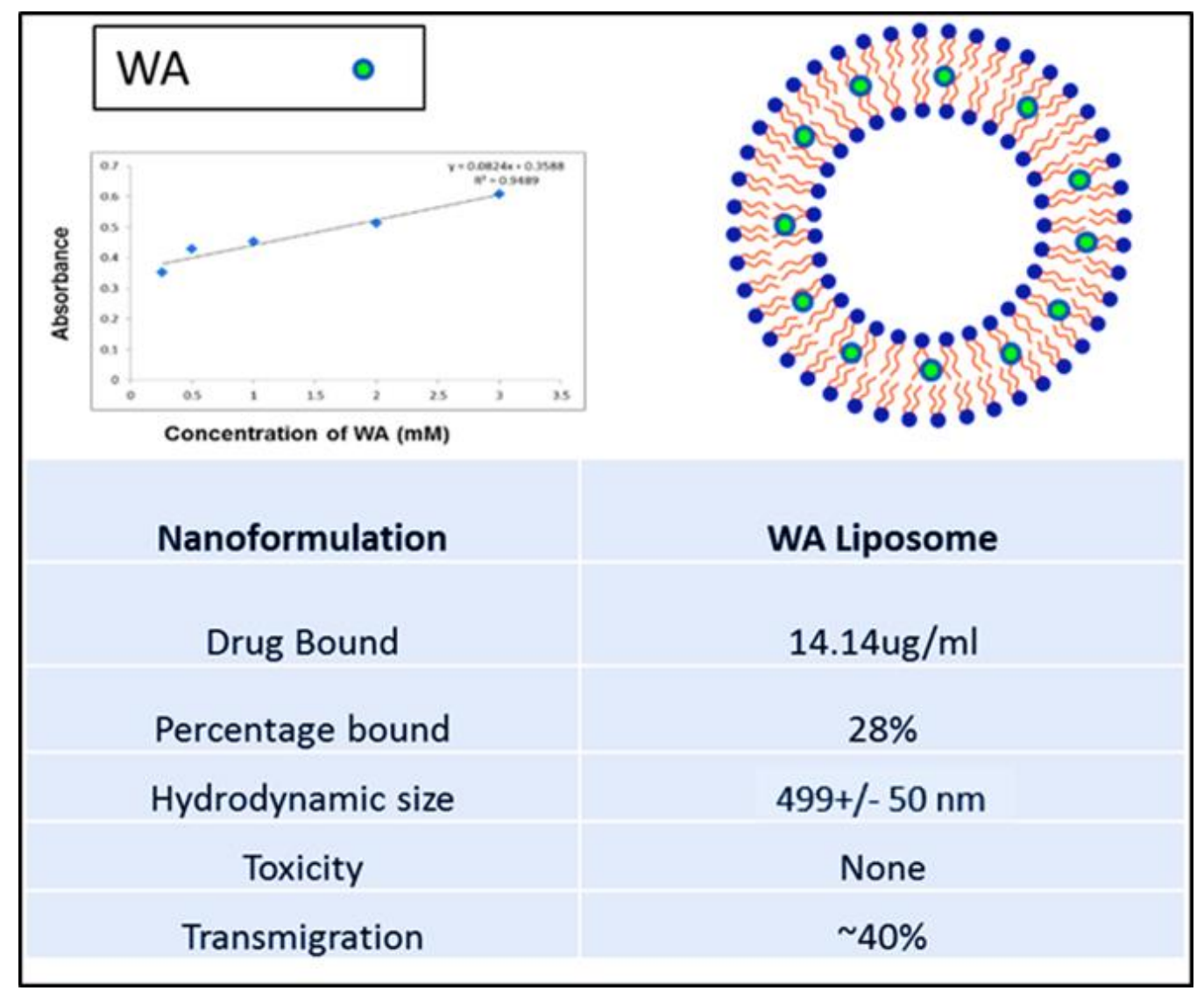




\subsubsection{Liposomal Nanoformulations are not toxic}

To evaluate the in-vitro cytotoxicity of the developed WA-LNF, we performed Trypan blue cell viability assay from the collected SHAPP cells treated with various concentrations WA-LNF. Our cell viability studies show that the Blank and drug loaded liposomes did not cause cytotoxicity, as the percentage of viable cells, were similar to untreated control up to $48 \mathrm{hrs}$ of treatment. They did not obstruct the cell viability and did not cause any toxicity to the cell cultures (Figure 4.5). Thus, signifying that all the tested doses of liposome NF were nontoxic and developed NF will not have any biocompatibility issues as the liposomes are made of biocompatible constituents.

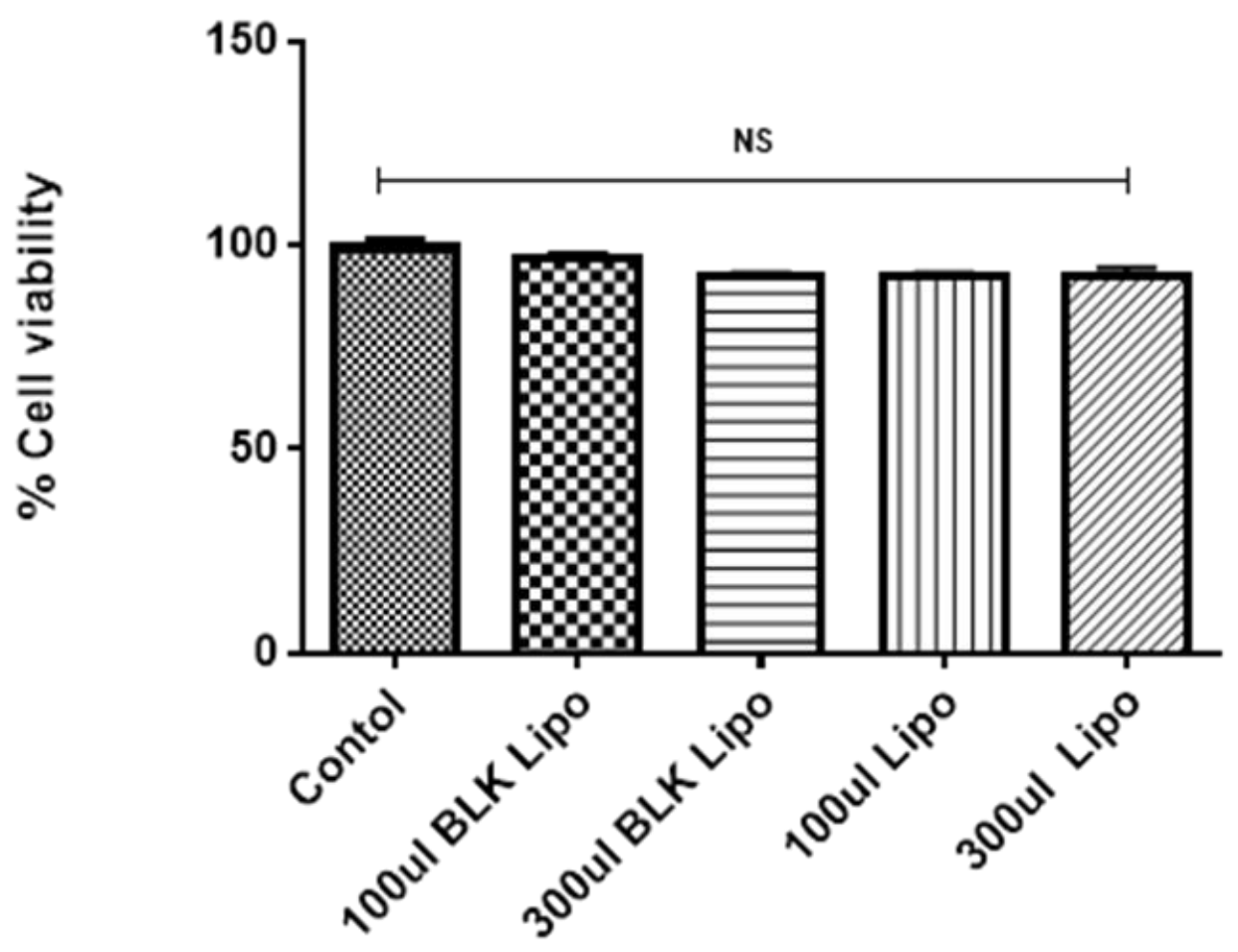

Figure 4.5 Cell viability test

The trypan blue viability test showed that the prepared liposomal nanoformulation were not having any effect on cell viability 


\subsubsection{Liposomal Nanoformulations transmigrate across the BBB}

The developed WA-LNF were added to the in-vitro BBB 3D model on the apical side of the trans-well. This setup was then incubated for 24 hours, TEER values were measured to insure that the WA-LNFs did not harm or distort the membrane integrity. FITC Dextran studies show that the $\%$ of transmigration was approximately $45 \%$ after the WA-LNF treatment. This ensured that the WA-LNF was able to transmigrate through the BBB (Figure 4.6).
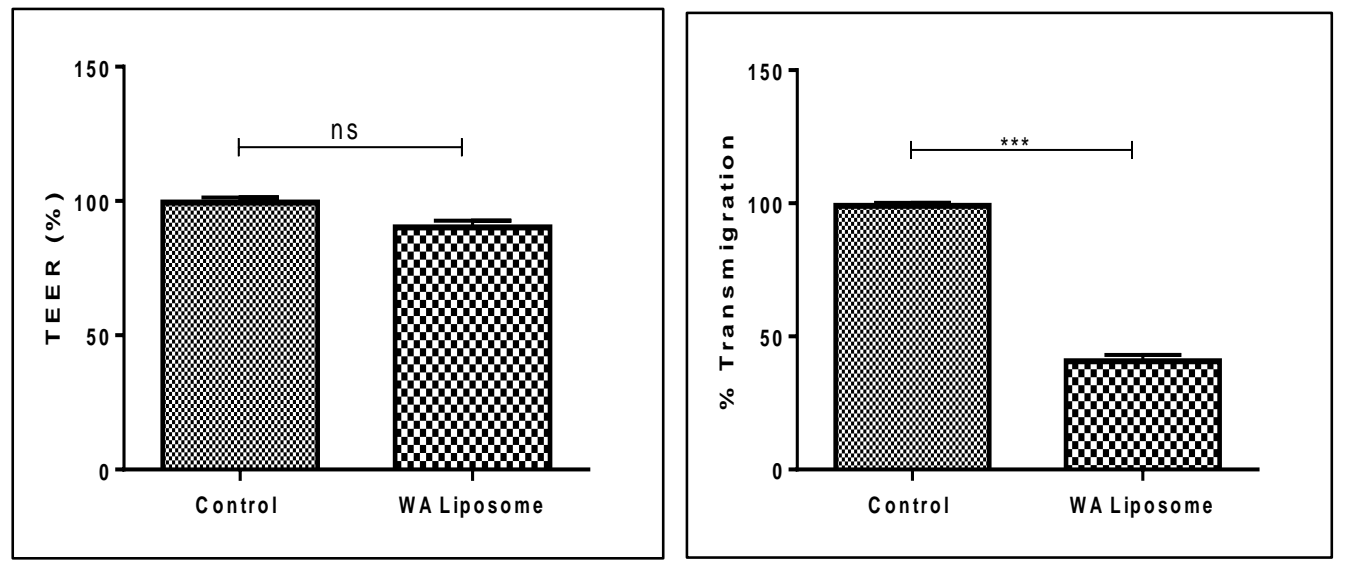

Figure 4.6 In-vitro WA Liposome transmigration and effect on BBB integrity compared to control

\subsubsection{Functional Efficacy of the WA-LNF upon BBB transmigration (ELISA)}

The efficacy analysis of the WA-LNF with respect to the cellular uptake and functional efficacy was performed. As explained before, the basal side of the BBB had SHAPP cells grown to full confluency. The SHAPP cells were harvested from the basal side of the transwells, the supernatant was collected after 24 hours of WA-LNF treatment. Control wells were treated by PBS. Supernatant were then analyzed by Human A 40 ELISA kit and compared to control. The standard curve was generated by utilizing different 
standards (different concentrations) and was utilized to calculate the concentration of A 340 in the sample supernatant. We observed approximately $40 \%$ reduction in the secreted A $A 40$ levels in the WA-LNF treated cell cultures compared to untreated control (Figure 4.7)

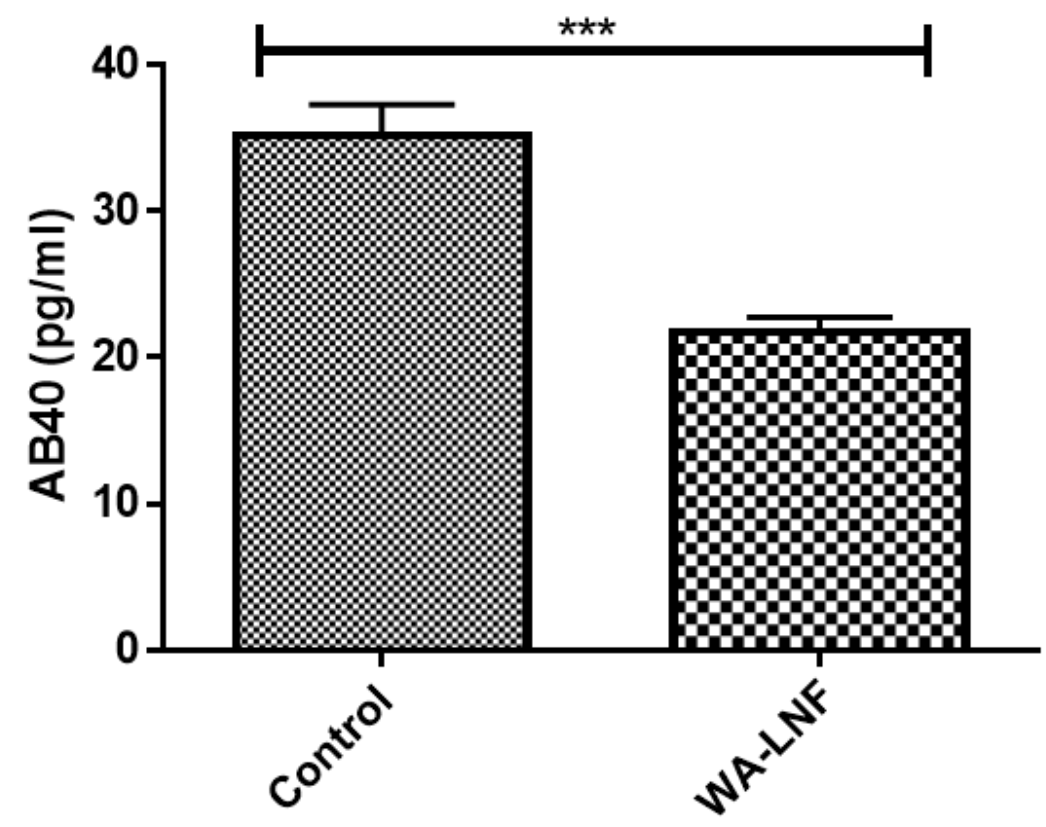

Figure 4.7 Effect of Liposomal WA across the BBB

WA-LNF decrease the AB40 levels across the BBB in the SH-APP cells, compared to untreated BBB associated SHAPP cells

\subsubsection{WA release study by membrane dialysis method}

Here we show a release pattern of the hydrophobic drug WA from liposomal Nanoformulation. The liposomes prepared were loaded with $100 \mathrm{mg} / \mathrm{ml}$ WA initially, and upon analyzing the unbound drug, we found that the drug bound to the liposomes was $\sim 29 \%(29 \mathrm{mg} / \mathrm{ml})$. Upon performing the membrane dialysis experiment with the drugloaded liposomes, we demonstrate that the cumulative release of WA was found to be 
$29.73 \mu \mathrm{g} / \mathrm{ml}$ until 24 hours. In this study, the surge of WA release was observed between 1-2 hours of the release, where a maximum of $4.5 \mathrm{mg} / \mathrm{ml}$ drug release was observed. Further, show that our liposomal nanocarrier displays a prolonged release pattern of $2+/ 1 \mathrm{mg} / \mathrm{ml}$ of WA until 48 hours (Figure 4.8 ).
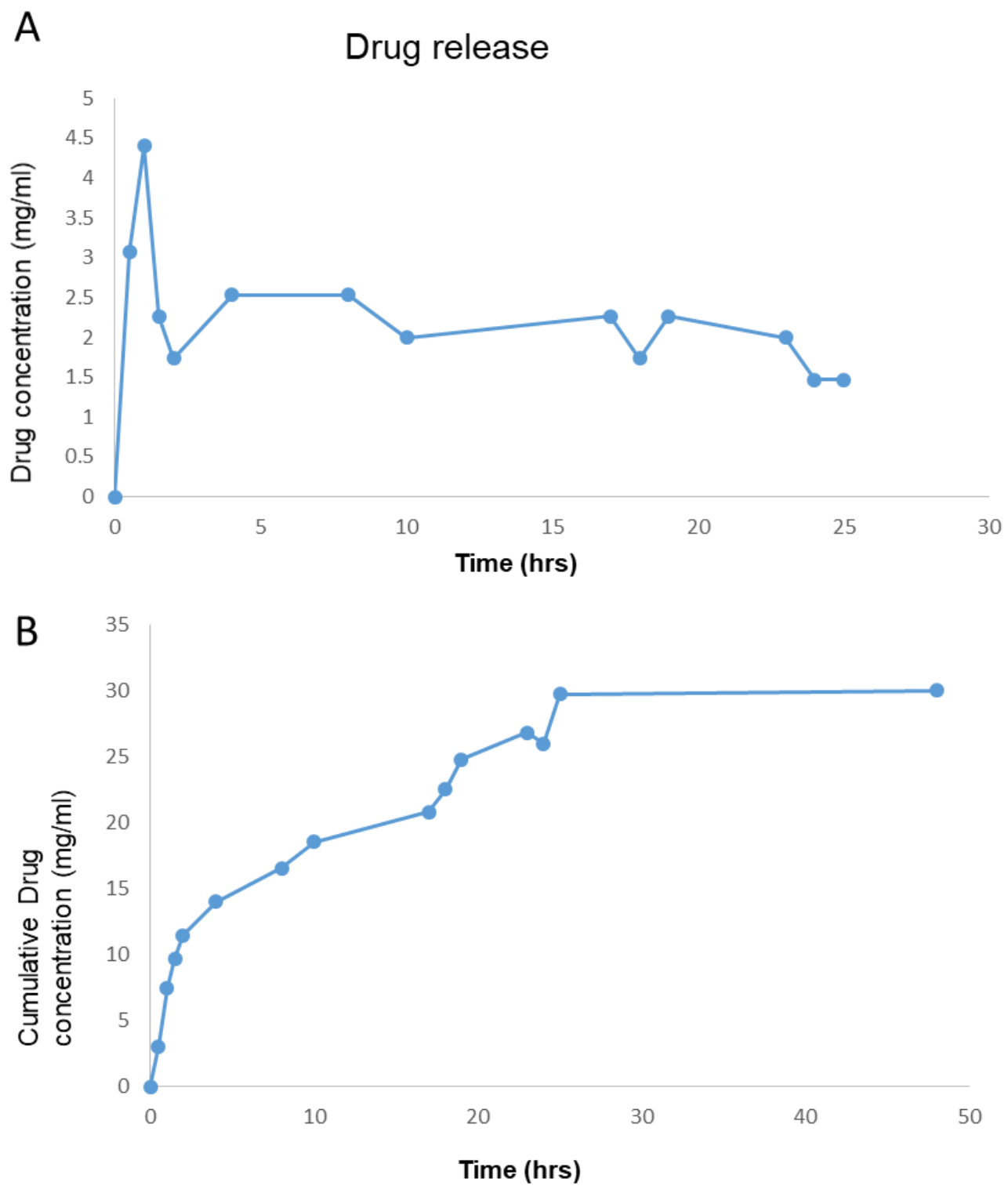

Fig 4.8 Drug release profile of WA

A) The graph demonstrates the concentration of WA release over the time of 48 hours. The surge of drug release was observed between 1-2 hours and later a prolonged release until 48 hours was observed. B) Cumulative drug release until $48 \mathrm{hrs}$. 
This design of liposomal delivery system for the hydrophobic drug molecule WA is promising as it has improved the encapsulation efficiency and enriched the drug retention time within the liposome. Therefore, it becomes utmost necessary to validate the release studies of WA from the liposome and the factors leading the encapsulation and retention/ release of WA. This membrane dialysis experiment shows an effective way of and release method using dynamic dialysis to study the liposomal membrane permeability of hydrophobic compounds.

\section{$\underline{4.4 \text { Discussion }}$}

In-vitro cell culture based studies, ex-vivo studies and clinical studies with respect to AD or AD-like neurological diseases indicate towards the heavy load of $A \beta$ deposition in the aged patients [292-295]. Amyloid deposition during disease progression covers almost the entire brain [296-299]. A $\beta$ plaques are reported to be heavily toxic to the CNS cells. The neuronal cells lose their communications, undergo oxidative stress and eventually lose their functions contributing towards disease pathogenesis. Therefore, targeting $A \beta$ in the brain could be of significant therapeutic advantage. The major shortcoming pertaining to CNS targeting drugs is the $\mathrm{BBB}$, which restricts the transport of most of the potential and vital drugs. Considering this problem, nanotechnology shows a promising potential in delivering drugs and targeting specific regions in the brain [109, $\underline{300}, \underline{301}]$. The field of nanotechnology is gaining more attention and is able to address more drug delivery and drug targeting challenges. The major attributes of nanomaterials and their conjugates like magnetic nanoparticles, magnetic-electronanoparticles, metallic nanomaterials, gold and silver nanoparticles, polymeric nanomaterials, metal-polymer nanocomposites, nanotubes etc. is their small size (in the range of nanometers). The inciting growth and clinical adoption of nanotechnology in the field of medicine is termed as nanomedicine. The 
important elements of nanotechnology are the minute size range, significant measurement and transformation at nanoscale and nanoscale specific functions over micro and bulk drug particles. The development of nanomedicine to the succession into clinical stage is very sensitive and complex process. Extreme preciseness and expertise is required to synthesize and characterize the developed nanoparticles, then studying its functional efficacy in-vitro and in-vivo, preclinical studies in non-human primates and then finally clinical trials. The nanomedicine or nanoparticles against diseases can be broadly categorized into (i) Polymer and liposome nanomedicine [302] and (ii) metal based nanomedicine [303-305]. Nanomedicine or nanotherapeutics is utilized against imbalance and abnormal disease factors in neurological disorders. Nanomedicine could be used for disease diagnostics as well as against neuropathological diseases, promotion of functional regeneration of damaged neurons, provide neuroprotection and facilitate drug delivery across the BBB.

Our laboratory is working in the field of nanomedicine delivery against infectious disease from several years and has demonstrated that anti-HIV drug loaded magnetic nanoparticle under the influence of external magnetic field can transport the anti-HIV drug across the BBB and can achieve the desired antiviral efficacy without inducing any cytotoxicity [ $\underline{108}$, $\underline{287}, \underline{288}, \underline{306]}$. The need of nanotechnological intervention in the field of drug development and delivery is extremely pressing in the current scenario. With the advent of various nanotechnological methods.

In this project, we have focused towards organic nanomaterials based medicine carriers i.e. liposomes to emphasize their advantages in context of therapeutics. Development of liposomes was intended primarily towards therapeutics and major attention was given 
towards their controlled size, stability, drug entrapment efficacy and sustained drug release [307]. The rationale behind utilizing liposomes in this study was to exploit the advantage of liposomal neuronal cells entry through the endocytic pathway [308-310]. We have employed liposomes as our drug delivery vectors due to the major qualities of the Liposomes. Liposomes are lipid bi-layered entities [311-313] and have an advantage over other nanoparticles as they can be loaded with both hydrophobic and hydrophilic drugs. The lipid bilayer of the liposomes captures the hydrophobic drug molecules. The liposomes are made up of biocompatible constituents like lipids and cholesterol. In this study, we are studying a novel formulation of phospholipid-based liposomes, which are made of egg a-phosphatidylcholine (amphiphilic molecule having a hydrophilic head and two hydrophobic polar chains) which is highly biocompatible, adaptable, stable, and forms bi-layered membranes when dispersed in aqueous or saline environment. Cholesterol, the second important constituent of developed liposomes, can facilitate the preferential transit of liposomal carrier through BBB and can be easily taken up by neuroblastoma cells. Liposomes are ideal drug carrier systems as their morphology is comparable to the cellular membranes, plus they can incorporate major variety of drugs.

Moreover, since various nanoparticles have large surface areas and have potential for drug delivery, the challenge arises during efficient loading of hydrophobic drugs, or high amounts of combination drugs on a single nanoparticle. Liposomes are promising, as loading hydrophobic drugs as well as combination drugs is easier and efficient in liposomes due to their hydrophobic as well as hydrophilic components. The liposomes can enter the cells in three ways, firstly they can be adsorbed by cell membrane and then lipase enzymes degrade their lipid bilayers and the active drug molecules are released and are diffused in cell membrane as well as cytoplasm. Secondly, liposomal membrane 
can directly fuse with the cell plasma membrane, releasing drugs into cytoplasm, and thirdly via receptor mediated endocytosis.

In this study, we were able to encapsulate the hydrophobic drug WA in the liposomes by dehydration method. Our results in this study demonstrate that WA-LNF had a hydrodynamic size of $\sim 450 \mathrm{~nm}$ in size and showed $28 \%$ drug binding capacity, with no cellular toxicity. The charge carried by the developed liposome was positive, therefore there was no aggregation of liposomes and due to positive $\zeta$-potential, and it was easier for the liposomes to interact with the cells. We also studied the BBB integrity by measuring the TEER and paracellular permeability using FITC-dextran transmigration of the developed NF. We demonstrated that the developed WA-LNF showed almost $50 \%$ transmigration across the BBB and did not disrupt the integrity of the $3 \mathrm{D}$ in-vitro BBB model. Next, we wanted to analyze the efficacy by which the liposomes entrap the drug and the continuous and sustained release of WA from the liposomal complex into the release medium. Our membrane dialysis study showed that the drugs start releasing from the liposomal complex within couple of hours and the dug release is continuous and stable. These liposomes cross the BBB either by absorptive-mediated transcytosis or receptor-mediated transcytosis, facilitated by the electrostatic communication between liposomal positive (cationic) charge and endothelial cells membrane's negative (anionic) charge [314]. WA liposomes could be of promising therapeutic importance. Therefore, in order to take this developed NF to further clinical levels, several optimization studies regarding the stability and toxicity plus efficient BBB transmigration, are needed. These liposomal nanoparticles are promising in applications such as age related or neuropathological diseases, which require the delivery of the drug across the blood brain barrier. The drugs available towards neurological issues are rejected due to 
pharmacotherapy failures like inadequate physical chemistry, minimal absorption, unfavorable pharmacokinetic parameters, instability and toxicity. This urges the need of the alternate medicine/nanomedicine. Further in vivo efficacy and drug delivery mechanistic studies are necessary to explore WA's therapeutic role in neurological disorders like HIV associated neurocognitive disorders and AD. Therefore, the WA Liposomal synthesized in our study have the potential of a combined therapy as a neuroprotectant and anti-inflammation molecule. It requires further studies to explore more in this field. 
CHAPTER 5: MOLECULAR BASIS FOR MID-REGION A $\beta$ CAPTURE BY LEADING ALZHEIMER'S DISEASE IMMUNOTHERAPIES: MECHANISM STUDIES

\section{$\underline{5.1 \text { Introduction }}$}

There is a constant speculation and scientific discrepancy behind the selection of main disease mechanism or the causal factor of $A D$ but the amyloid hypothesis is the most important and accepted one. In the amyloid hypothesis, AD is caused by excessive accumulation of the $A \beta$ peptide leading to the plaques. Amyloidogenesis has been associated with a broad spectrum of diseases in which amyloid protein is invariably misfolded and deposited [1] [ $\underline{315}, \underline{316}$ ] [317]. The monomers react with neurotoxic oilgomers and aggregate into multimers, fibrils and finally fibrillary aggregates (Figure 5.1). The multiple structural forms of $A \beta$ interact and associate together forming big and insoluble plaques. Therefore, in order to understand and evaluate the ability of WA chemical structure to physically interact with $A \beta$ protein structure (monomeric and multimeric), in this chapter, we have focused on the physical interactions of $A \beta$ with WA to understand the mechanism of WA working in the disease pathway. 


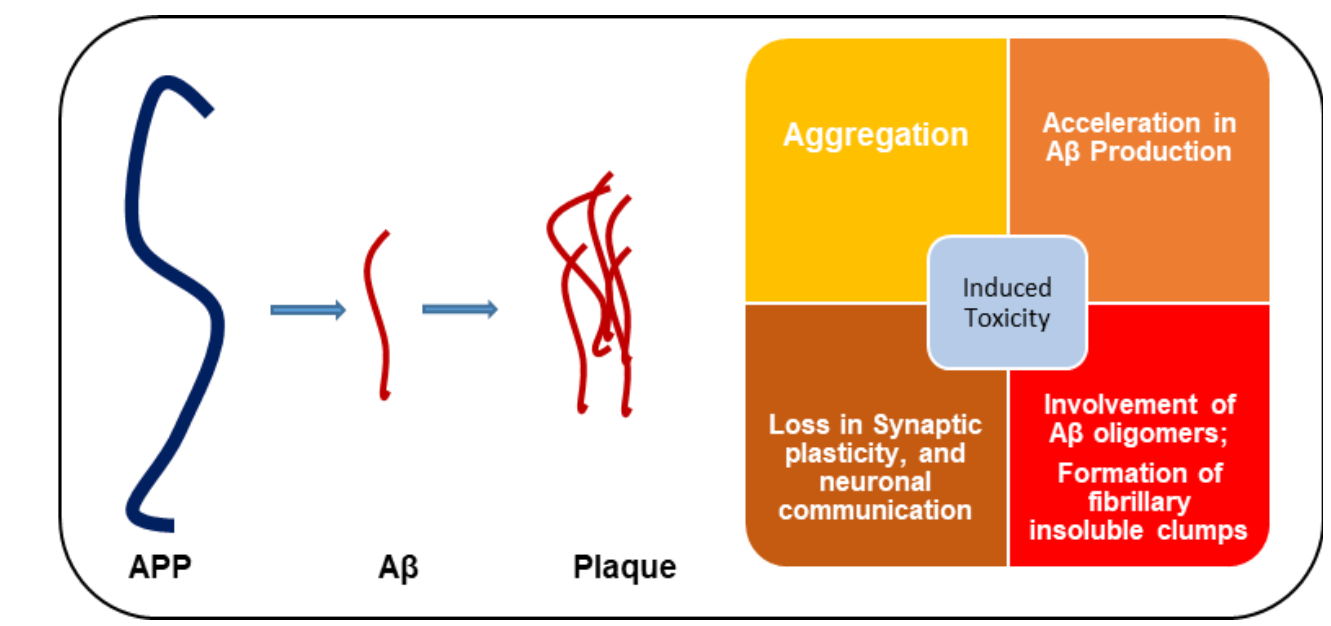

\section{Figure 5.1 The stages of amyloid fibril formation and the associated cellular toxicity}

Before studying the interaction of $A \beta$ directly with WA, we focused on elucidating the role of WA and its effect on the enzymes involved in $A \beta$ processing, i.e. $\beta$ and $\gamma$ secretases. These enzymes are extremely important in APP processing during amyloidogenesis, to release $A \beta$ that is why we speculated that WA might have a direct effect on these enzymes as well. We designed an experimental study to have a deeper understanding towards mechanism of action of WA by measuring its effect on $\beta$ and $y$ secretases. Even though we were able to demonstrate the role of WA in decreasing the secreted $A \beta$ in our preliminary in-vitro study, we were not able to observe effect of WA on $\beta$ and $y$ secretases enzymes. This could be due to low amounts these enzymes secreted by the SHAPP cells, which went undetectable by the ELISA technique. We plan to do in-vivo and in-silico studies to address this question. Therefore, in this thesis currently, we hypothesize that that the main target of WA is $A B$ monomers and aggregates, and WA may be highly interactive with the specific regions of $A \beta$ proteins, for which we further performed in-silico studies to support our hypothesis. 
$A \beta$ peptide is a sticky peptide which is abundant in the brain plaques, and its abnormal accumulation of $A \beta$ in $C S F$ and plasma $[19, \underline{318}]$ result due to biochemical abnormalities caused by APP mutations [292]. This concept has been well reported by study incorporating 3D human neural cell culture model to demonstrate $A \beta$ accumulation [319].

There are various strategies studied by researchers globally, where the popular approach towards $A D$ is the targeting strategy of the various antibodies being developed as immunotherapies. Antibodies are considered amongst the most promising approaches for the treatment and prevention of $A D$ and $A D$ related diseases. The promising Solanezumab (Eli Lilly) and crenezumab (Genentech) were humanized monoclonal antibodies targeting the mid-region of the neurotoxic $A \beta$ peptide [320] an early biomarker of $A D$ pathology and the major component of plaques found in AD-affected brain. Results of large-scale phaseIII clinical trials of solanezumab, and clinical anti-A $\beta$ antibody called bapineuzumab (Pfizer, Johnson \& Johnson) in patients with mild to moderate AD were reported in 2014. Both studies concluded that treatment did not improve clinical outcomes in AD patients.

Unlike solanezumab, bapineuzumab demonstrated target engagement in ApoE4 carriers, lowering brain amyloid and hyperphosphorylated-tau (the constituent of tangles) and total tau levels in cerebral spinal fluid relative to placebo [321]. The failure of bapineuzumab and solanezumab to improve clinical outcomes is considered by many to be a question of treatment window since deposition of amyloid in the brain can predate symptomatic dementia by decades [322]. Thus, clinical trials examining anti-Ab antibody treatment in at-risk, asymptomatic individuals are planned or underway. These include the antibodies solanezumab (in the Anti-Amyloid treatment in Asymptomatic Alzheimer's disease (A4)

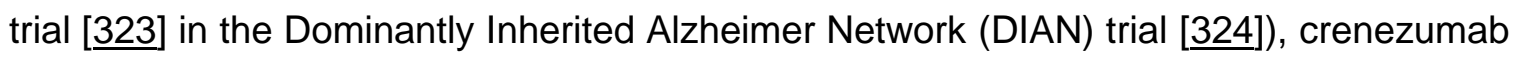


(in the Alzheimer Prevention Initiative (API) trial [325]) and gantenerumab (Chugai/Hoffmann-La Roche - in the DIAN trial).

The murine parent antibody of the humanized monoclonal antibody solanezumab, 266 is reported to target $A \beta$ within residues 13-28. It becomes important to understand $A \beta$ engagement with the clinical candidates for facilitating the development of active A $\mathrm{A}$ directed therapies that can be drug therapy (naturally acquired plant based drugs like ASH and Curcumin) or it can be immunotherapy (vaccines). Studies demonstrating the involvement of mid region of $A \beta$ employed crystallized recombinant solanezumab Fab fragment complexed to the mid-region of the $\mathrm{Ab}$ peptide and determined its structure to a resolution of $2.4 \mathrm{~A}^{\circ}[326]$. This study showed the conformation adopted by the Ab peptide in the antibody-binding site. They observed unambiguous electron density across $A \beta$ residues 16-26 (KLVFFAEDVGS) in the most complete model of solanezumab.

Earlier, anti-N-terminal antibody holding $A \beta$ in an extended coil over the first eight residues were reported [327-330] Antibody like bapineuzumab and its murine parent 3D6, showed the $\mathrm{N}$-terminal five residues of $A \beta$ captured [331]. Additionally, ponezumab (Pfizer) antibody with specificity for the C-terminus of $A \beta 40$, was demonstrated to attach to the highly hydrophobic region in an extended coil conformation [332]. The structural studies of anti-A $\beta$ structures, provides a foundation for the designing of upcoming and next generation immunotherapies to lower cross-reactions and more interaction with the peptides. The understanding of peptide or protein structures may also help in the identification of alternative mechanisms of action of immunotherapies or drug therapies, by exploiting the proteins sharing elements of the $A \beta$ epitope. 
The majority of patients with $A D$ suffer from impaired cerebral circulation. $A \beta$ multimerizes and interacts with $A \beta$ fibrils and $A \beta$ oligomers act as seed to further accumulate the fibrils into multimers and clump, forming clearance-resistant abnormal aggregates or plaques. Since immunotherapy candidates like solanezumab, and crenezumab failed the clinical trials. It becomes imperative to unfold and dig new tangents of therapy. The natural components derived from the plants and plant products become important candidates in targeting $A \beta$ during $A D$ pathogenesis. Therefore, we chose as a neuroprotectant and studied its interaction with the middle region of $A \beta$, like Immunotherapy candidates like solanezumab. Current advances in bioinformatics and drug development made it easier for us to strategize the in-silico experiments. Upon understanding the $A \beta$ hypothesis and we were interested in understanding the targeting mechanisms of the WA drugs against $A \beta$. We assessed WA's interaction with the peptide and focused on analyzing the targeting specificity to strengthen the importance of WA as a promising drug candidate against the $A \beta$ fibrils and their aggregated complex and insoluble structure.

The in-silico molecular dynamics structural studies capable of designing protein 3D models and estimating drug binding with the antigens or the protein of interest is currently one of the most desirable techniques. In the field of drug discovery and targeting, the method of finding the best-suited drug to aim at target protein is termed as molecular fishing (Figure 5.2). There are experimental methods of direct molecular fishing developed for identification of potential partners of protein-protein and protein-peptide interactions. The experiments are based on surface plasmon resonance technology (SPR) studies of the molecules, size exclusion and affinity chromatography techniques and identification of proteins by employing mass spectrometry (LC-MS/MS). However, in this project there was no requirement of the molecular fishing as we already have established the role 
of WA against $A \beta$ and we performed in-silico molecular simulation to support our results and elucidate the mechanism involved.
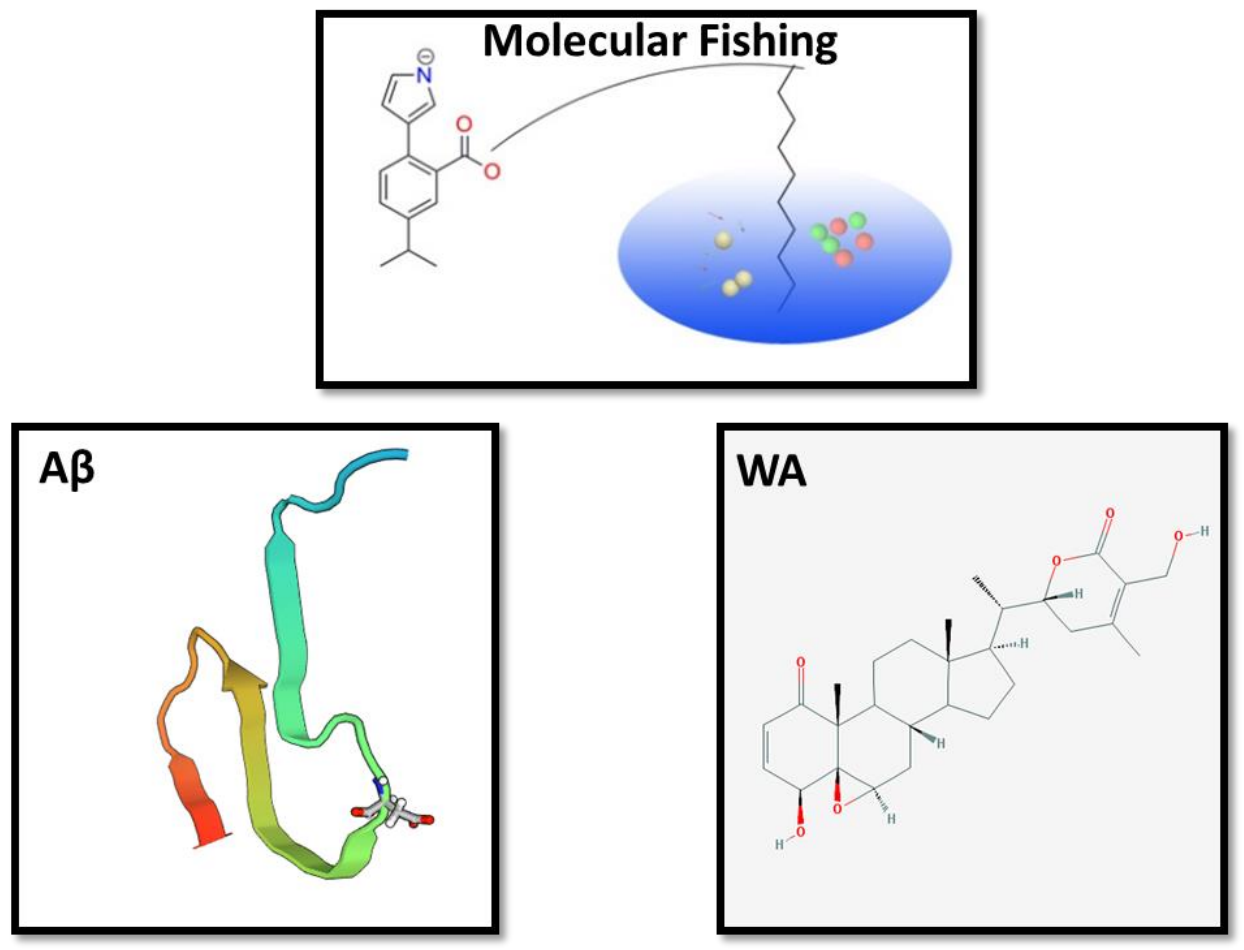

Figure 5.2 Molecular Fishing

The study of deducing best-fit drugs against a target protein or antigen based on their structural interactions. The drug candidate interacting with the target utilizing the least energy structural modifications are chosen to be the bestsuited drug molecule against the protein

Further, we have demonstrated the structure of the $A \beta 42$ protein molecule in the water environment. Every protein exists in a particular environment, and can reach its maximum stable structure within a medium or an environment like saline or water. Here we demonstrate that the PDB model when generated and visualized in a water environment takes proper structure of $A \beta 42$ protein (Figure 5.3). 

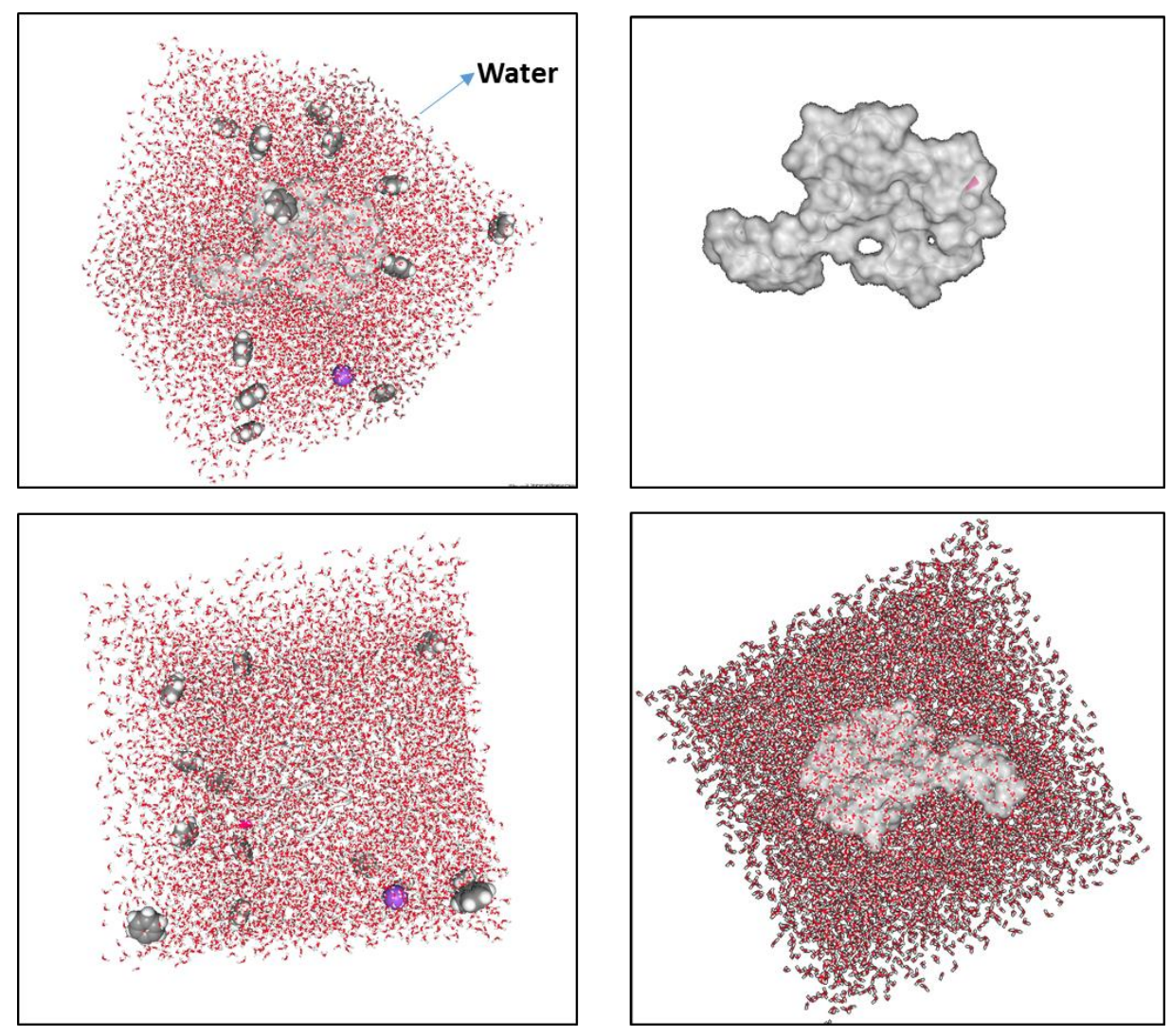

Figure 5.3 Molecule in a water environment.

The figure demonstrates the $3 D$ crystal structure of $A \beta$ molecule in water environment

In this study, we investigate the structural details of the $A \beta-W A$ interaction. We identified the middle region of $A \beta 42$ as the most critical region for the interaction, which can be targeted by drugs. WA binding to this region may block further aggregation or addition of new peptides or may break the mature insoluble plaques. Overall, our study elucidates the $A \beta-W A$ interaction and clarifies the mechanism by which WA binding depletes $A \beta$ aggregation and further plaque formation. These results may facilitate the effective therapeutics against plaque aggregation leading to the treatment of neurotoxicity and cerebrovascular abnormalities in AD. 


\section{$\underline{5.2 \text { Materials and methods }}$}

\subsubsection{Estimation of Protein Structure}

The human crystal structure of Monomorphic A 342 amyloid Fibrils (PDBIDs: 5KK3) [333] was retrieved form the Protein Data Bank (PDB) (http://www.rcsb.org). Additionally, A $\beta$ monomer protein was modelled by Amber Molecular Dynamics Software, Version 14. The energy minimization of target proteins was conducted by using online tool Sander package of AMBER14 resolve the stearic clashes from protein structures. By using, 3D structure of Alzheimer's $A \beta$ Chain $A$ of monomorphic $A \beta 42$ (5kk3.A.pdb) as a template with an $E$ value of $4.3 e-16$ and a score of 63 , we modelled this protein structure.

\subsubsection{Candidate Structure}

The steroid lactone WA was selected as the drug of interest for present study. The 2D structure of WA drug was derived from Pubchem Open Chemistry Database the ACD/ChemSketch tool. (Pubchem ID: 265237). A detailed compound summary of chemical and physical properties and of WA were retrieved from PubChem database.

\subsubsection{Molecular Docking of WA with A $\beta 42$}

Further, WA was docked with $\mathrm{A} \beta 42$ by Rosetta, a software suite that includes algorithms for computational modeling and analysis of protein structures. The avenger binding affinity of WA cluster of 100 decoys in the common binding pocket was calculated to be approximately -8.12 REU.

\subsubsection{Molecular Dynamics Simulation}

To investigate the role of WA on destabilization of $A \beta$ fibrils, we performed extensive $\mu S$ length all-atom molecular dynamics (MD) simulations using NAMD [3ㅜㄴ with Charmm36 
force field [335]. We performed extensive $\mu$ S-length simulations to investigate the fibril disruption and growth inhibition by WA. The $A \beta$ fibrils exist in several polymorphs with different interfacial interactions between protofilaments [336-339]. Because of the different local arrangements $A \beta$ have potential implications for biological activities [ $\underline{340}$ ], therefore, we have considered different fibrillar structures of both $A \beta_{1-42}$ ( $p d b$ codes $50 Q V, 5 K K 3$, 2NAO) and A 340 (pdb id 2M4J, 2LMP, 2MVX). To incorporate local fluctuations in fibril conformations, we implemented a short 100-ns MD simulation for each of these fibril structures. From each run, about 1,000 conformations were sampled, making a pool of 6,000 A $\beta$ fibril conformations. WA was screened against these sampled $A \beta$ conformations using AutoDock Vina [341] and custom scripts [342]. About 100 top-ranked WA-A $\beta$ fibril complexes from the docking results were analyzed to filter and identify consensus bestranked binding poses. About ten complexes thus identified were used to set up MD simulations.

\subsubsection{Protein Structure Visualization and analysis}

The generated protein structures from the Docking experiment were complex and visualized by Chimera $X$ protein structure visualization software, which shows the 3D structure of the protein and the specific regions of drug binding. This tool provides several command tools to view the protein structures, their surfaces, and the intra and inter bonding between protein-protein or protein-drug interactions.

\section{$\underline{5.3 \text { Results }}$}

As we have established in this thesis, that WA has a direct effect on A $\beta$ levels, we wanted to further investigate the mechanism of underlying process by which WA might be 
interacting with the Amyloid protein. It was important to address the WA binding/interaction with $A \beta 42$ and the precise region of binding, and structural modification in protein, if any.

\subsubsection{Physical and Chemical properties of WA compound}

WA is purified compound derived from from root extract of the medicinal plant Withania Somnifera known for its importance in the field of Ayurveda medicine. WA molecule is a size-excluded product from parent compound $\mathrm{ASH}$, and is small in size. WA has been studied well in various fields research and medicine field including cancer and angiogenesis. In order to utilize WA for the in-silico study, we focused on detailed characteristics of WA's physical and chemical properties, which are available at Pubchem website (Table 5.1) 
Table 5.1 Characteristics of Withaferin A

\begin{tabular}{|c|c|}
\hline Chemical Name & Withaferin A \\
\hline MeSH Entry Terms & $\begin{array}{l}\text { 5,6-epoxy-4,22,27-trihydroxy-1- } \\
\text { oxoergosta-2,24-dienoic acid delta- } \\
\text { lactone }\end{array}$ \\
\hline Molecular Formula: & C28H3806 \\
\hline Molecular Weight: & $470.606 \mathrm{~g} / \mathrm{mol}$ \\
\hline \multicolumn{2}{|l|}{ 2D structure } \\
\hline \multicolumn{2}{|l|}{ 3D Conformer } \\
\hline Canonical SMILES & $\begin{array}{c}\mathrm{CC} 1=\mathrm{C}(\mathrm{C}(=0) \mathrm{OC}(\mathrm{C} 1) \mathrm{C}(\mathrm{C}) \mathrm{C} 2 \mathrm{CCC} 3 \mathrm{C} 2(\mathrm{CC} \\
\mathrm{C} 4 \mathrm{C} 3 \mathrm{CC} 5 \mathrm{C} 6(\mathrm{C} 4(\mathrm{C}(=0) \mathrm{C}=\mathrm{CC} 60) \mathrm{C}) 05) \mathrm{C}) \\
\mathrm{CO}\end{array}$ \\
\hline Hydrogen Bond Donor Count & 2 \\
\hline Hydrogen Bond Acceptor Count & 6 \\
\hline Topological Polar Surface Area & $96.4 A^{\wedge} 2$ \\
\hline Heavy Atom Count & 34 \\
\hline \multicolumn{2}{|l|}{ Crystal Structure } \\
\hline Ionization Mode & Positive \\
\hline
\end{tabular}




\subsubsection{Structural studies}

Our structural studies showed that WA has the capacity to interact and bind to the hydrophobic middle region of the $A \beta 42$. This middle region is reported to be interactive by various other studies, which reported the interaction of clinical immunotherapy agents/ antibodies promising for immunotherapy, with the middle region of $A \beta[\underline{320}, \underline{343}$ (as discussed in Section 5.1). Upon docking the neuroprotectant drug WA with the generated structure of $A \beta 42$, we established that WA binds to the $A \beta$ protein firmly in the middle region of the protein (Figure 5.4). The drug sits precisely in the hydrophobic pocket of A 442 protein. This interaction of WA with $A \beta 42$ did not distort the chemical structure of WA, as it maintained the stability of the WA drug. The amino acid motif involved in the binding with the WA molecule was "VFAEDVGS" which constructs the mid-region A $\beta$. This result was fascinating as WA is a hydrophobic drug, we were expecting its obvious interaction with the hydrophobic part of the protein, to acquire safe hydrophobic environment, which will not distort its chemical structure. The representative figure of protein structure complexed with WA shows position of single amino acids (one letter code labelled) and shows the placement of WA near the FAEDVGS motif (Figure 5.5). Amino acids in the hydrophobic core are sequenced as VFAEDVGS ( $F=$ Phenylalanine, $A=$ Alanine, $\mathrm{E}=$ Glutamic acid, $\mathrm{D}=$ Aspartic Acid, $\mathrm{V}=$ Valine, $\mathrm{G}=\mathrm{Glycine}, \mathrm{S}=\mathrm{Serine})$. These amino acids help in facilitating the interaction between protein and drug, leading to potent protein-drug interactions. 


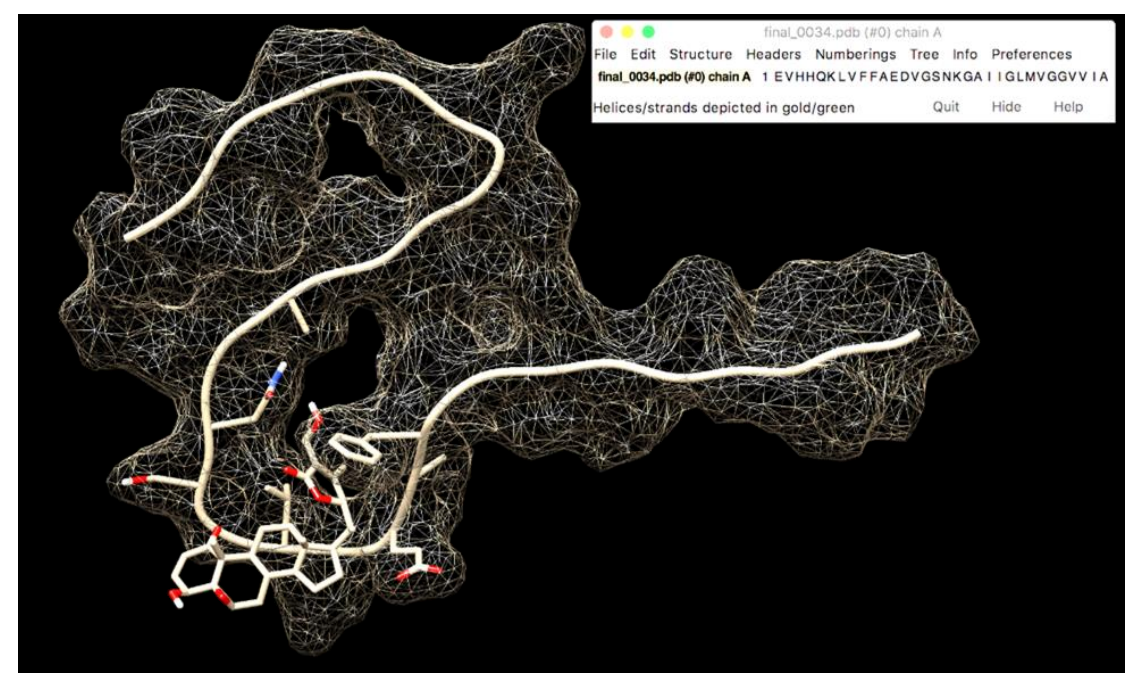

Figure 5.4 Visualization of protein structure interacting with WA

Visualization of the generated protein drug complex with the help of Chimera X, visualization software. The pdb file generated by the docking experiment was uploaded in the ChimeraX software. This figure demonstrates the Mesh view of the $A \beta 42$ protein (golden ribbon) with WA (Red, Blue and Gold sticks chemical structure).

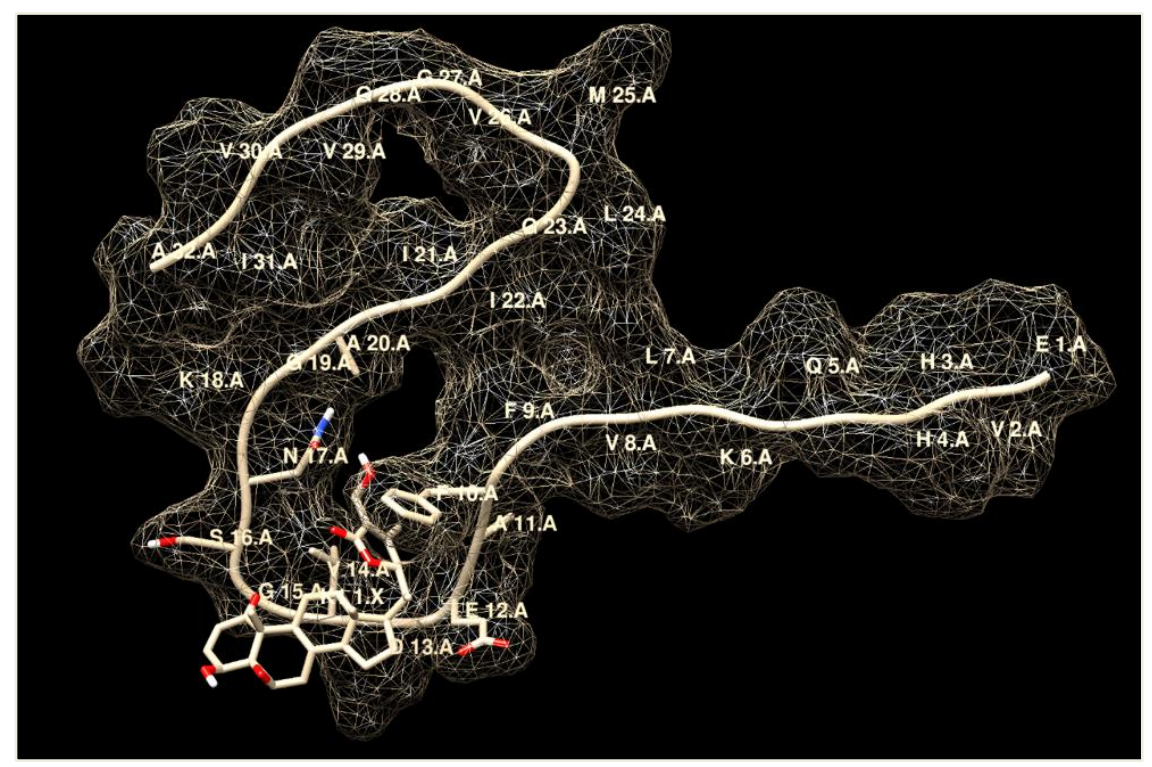

Figure 5.5 Visualization of the amino acid labelled protein drug complex

The pdb file generated by the docking experiment was uploaded in the ChimeraX software. This figure demonstrates the Mesh view of the $A \beta 42$ protein (golden ribbon) labelled with one-letter amino acid code, and its interaction with Withaferin A (Red, Blue and Gold sticks chemical structure). (Sequence:DAEFRHDSGYEVHHQKLVFFAEDVGSNKGAIIGLMVGGVVIA 


\subsection{3 $A \beta$ has an interactive hydrophobic motif}

Upon deriving, the interaction site of WA with the hydrophobic middle region of the protein (Figure 5.6) and labeling the amino acids in the hydrophobic core sequenced as VFAEDVGS. The figure demonstrates the mesh view of the $A \beta 42$ protein (golden ribbon) labelled with one-letter amino acid code, and the highlighted (Teal) mid-region of the protein. It shows the interaction of WA (Red, Blue and Gold sticks chemical structure) with the mid- region (Teal) (hydrophobic pocket) of the $A \beta 42$ protein. It is a clear representation of protein-drug interaction. $A \beta$ capture by the neuroprotective agent is explained here with the reported mid-region A $\beta$-WA complex of the generated drug-protein in-silico structure. Additionally, the protein surface view presentation (Figure 5.7) shows that WA interacts with the hydrophobic pocket which has minimal stearic hindrance. The minimal stearic hindrance provides a potent area or a sort of pocket for WA to bind, without loosing its benzene rings, or getting distorted. Visualization of the generated protein drug complex with the help of Chimera $X$ visualization software, demonstrates the surface view_of the A 342 protein (golden ribbon) labelled with one-letter amino acid code, and its interaction

with WA (Red, Blue and Gold sticks chemical structure). WA interacts with the mid- region (Orange arrows) (hydrophobic pocket) of the $A \beta 42$ protein. 


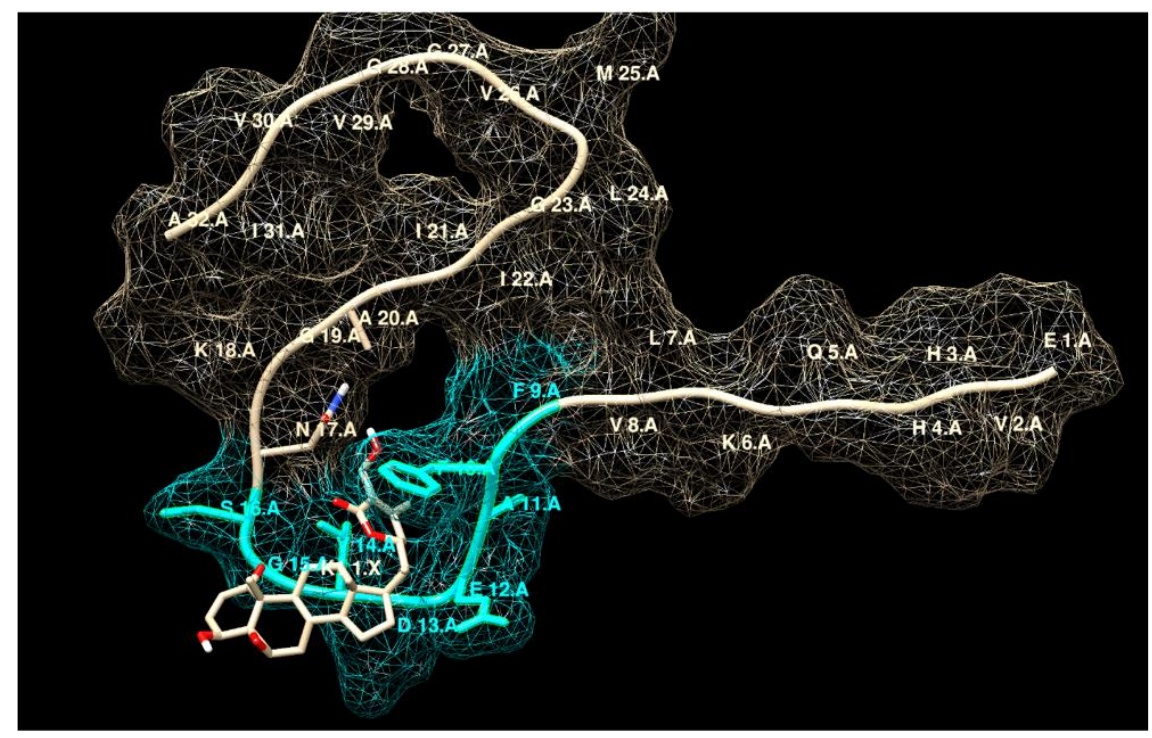

Figure 5.6 Mesh view of the Aß42 protein (golden ribbon) and its interaction with WA

Red, Blue and Gold sticks represent the WA chemical structure and the highlighted Teal colored section shows the hydrophobic middle region of the $A \beta 42$ protein, which contains FAEDVGS motif

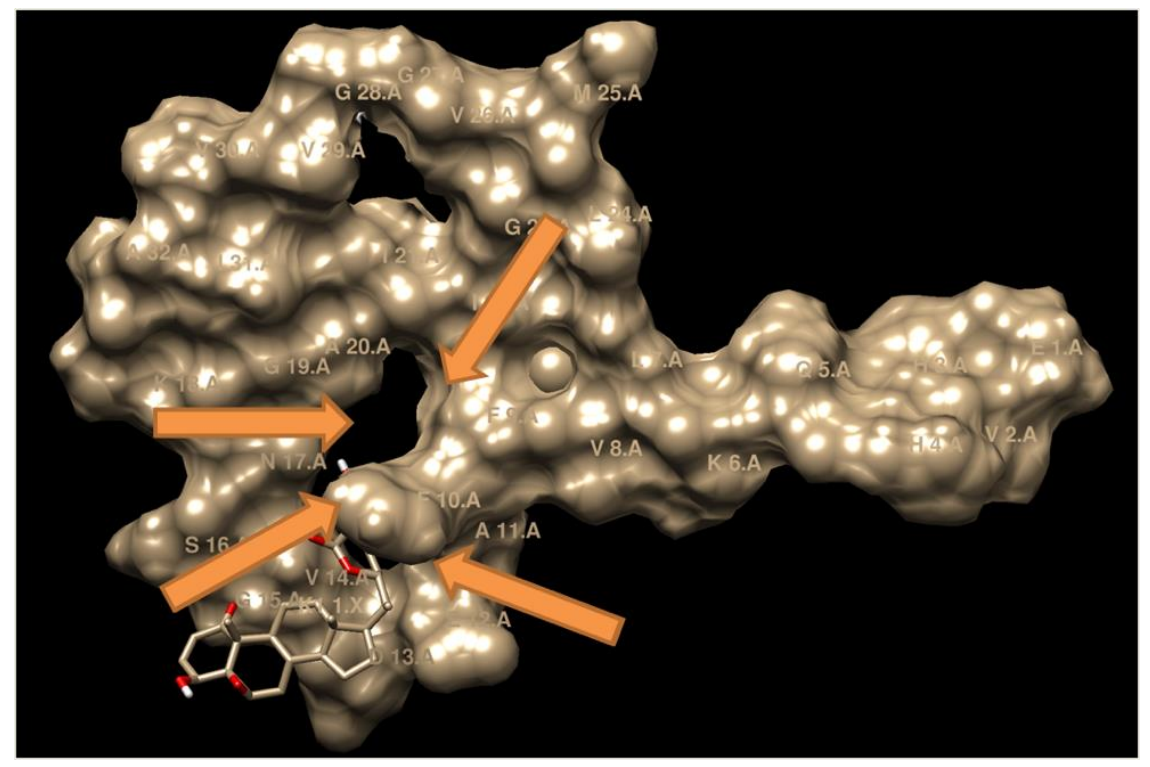

Figure 5.7 Surface view of the $A \beta 42$ protein (golden ribbon) and its interaction with WA Red and Gold sticks represent WA and show its interaction with the mid- region (Orange arrows) (hydrophobic pocket) of the golden surface of $A \beta 42$ protein 


\subsubsection{Drug-protein interactions via Hydrogen bonding and the binding is stable and specific}

Furthermore, we wanted to study the type of bonding if any between these two moieties. Upon selecting the probable hydrogen bonds by the Chimera software interface, made between drug and protein, we were able to show the presence of 3 significant Hydrogen bonds made by WA with $A \beta 42$. The best-docked energy complexes were further deep analyzed based on hydrogen and hydrophobic interactions pattern between ligand and target protein. The active binding region of $A \beta$ was hypothesized to be the middle region based on literature involving immunotherapies and their interaction with amyloid peptides. Focusing on the hypothesis when we ran our experiments, our results proved our hypothesis and showed that WA binds within the active region of target protein by forming couple of hydrogen bonds. Figure 5.8 A,B demonstrate the closer look of the mid region of $A \beta 42$ protein and show three specific hydrogen bond interaction of WA with the amino acids ASN17, GLY15 and SER16 of the protein, from a slightly different angle. The structure activity relationship (SAR) analysis shows that WA forms three hydrogen bonds at specific residues (ASN17, GLY15 and SER16) with target protein. The OH- (anions) (hydroxyl group) had a strong interaction with the $\mathrm{H}+$ (cations) of the three amino acids

namely ASN17, GLY15and SER16 (Figure 5.8 A, B) These interacting residues Asn $\left(\mathrm{C}_{4} \mathrm{H}_{8} \mathrm{~N}_{2} \mathrm{O}_{3}\right)$, Gly $\left(\mathrm{C}_{2} \mathrm{H}_{5} \mathrm{NO}_{2}\right)$ and $\operatorname{Ser}\left(\mathrm{C}_{3} \mathrm{H}_{7} \mathrm{NO}_{3}\right)$ have molar masses of $131.12 \mathrm{~g} / \mathrm{mol}$, $75.07 \mathrm{~g} / \mathrm{mol}$ and $105.09 \mathrm{~g} / \mathrm{mol}$ respectively. They are studied to be significant in the downstream signaling pathways. 



Figure 5.8 Demonstration of Hydrogen bonding between $A \beta 42$ and WA

A) Three Hydrogen bonds are formed between WA and the A $\boldsymbol{\beta}$ (Yellow lines) giving stability to the binding structures, B) The figure in the zoomed-in area of the mid region of $A \beta 42$ protein and shows three specific hydrogen bond interaction of WA with the amino acids ASN17, GLY15 and SER16 of the protein. 


\subsubsection{Dynamic modelling: Speculating the mechanism of action of WA}

Our preliminary computational work on WA binding to $A \beta$ fibril has shown that WA binds in two physiologically relevant sites and affects the fibril growth and stability. Specifically, WA can successfully recognize and insert through the loop segment between residues 24-33 in the $A \beta$ fibril. This insertion of WA into the $A \beta$ fibril destabilizes the fibril, suggesting that it can potentially destabilize the fibrillar structures. Another binding site is on the fibril interface and this can potentially inhibit the fibril growth. We will perform extensive $\mu \mathrm{S}$ length simulations to investigate the fibril disruption and growth inhibition by WA (Figure 5.9).

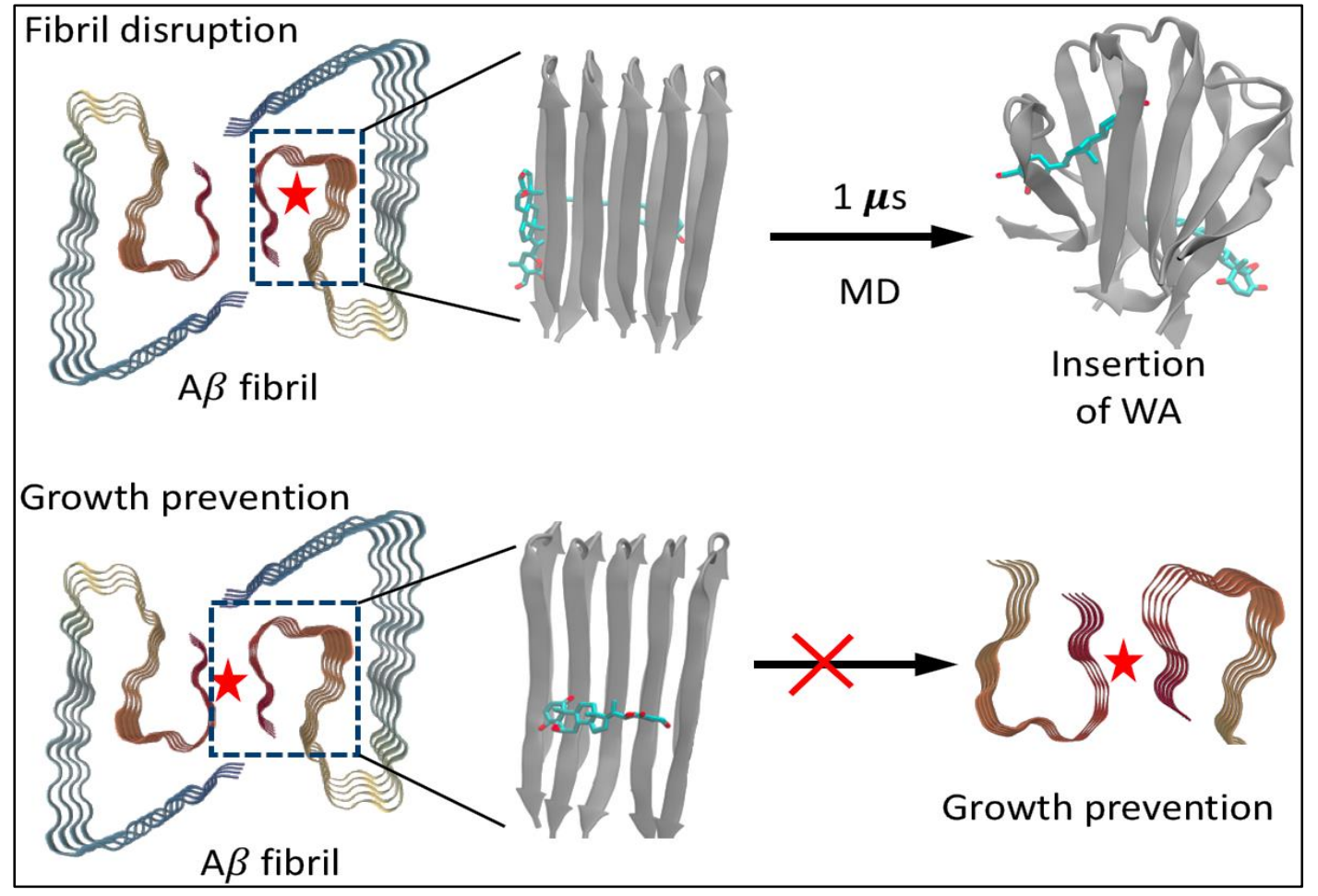

Figure 5.9 Two proposed mechanisms of WA's interaction with Aß42 fibrils 


\subsubsection{Protein drug binding is stable and specific with time lapse}

Upon the time-lapse study of the interaction of two representative WA molecules with the matured fibrils of $A \beta 42$, we report constant movement of WA between the fibrils and then eventually settling down in the middle portion of the proteins, behaving similar to what we showed in our monomer structure depiction studies (Figure 5.10).

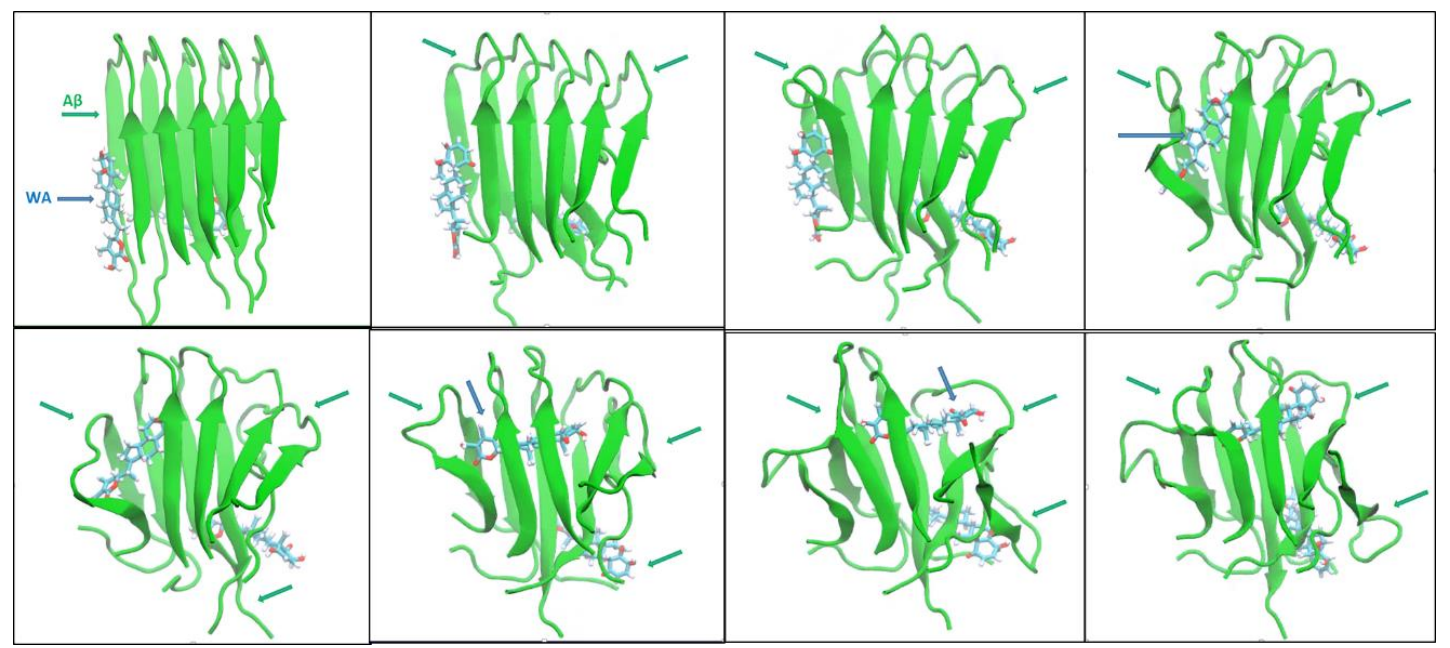

\section{Figure 5.10 WA interaction with $A \beta 42$ is stable}

These structures are the stills from the time-lapse video of interaction of WA molecules with $A \beta 42$ protein, which shows that WA remains interacting with the protein for a longer duration, and with stability

\section{$\underline{5.4 \text { Discussion }}$}

In the era of increasing neuropathological issues and complexities and lack of efficient drug targets, it becomes important to understand the structure and function of the target disease causing protein to generate the minimal free energy structures and identify the most potent target in-silico before attempting the in-vivo or clinical attempts of testing the drug efficacy. The advantage of in-silico study is that it gives us an opportunity to explore thousands of drug candidates and study their interaction with the target proteins, enzymes 
or receptors, virtually in a saline or water environment. In-silico study are extremely efficient, accurate and financially minimal, and saves valuable time of the researchers. The experiments deliver top 5 or 10 minimal free energy protein structures and potent drug candidates to initiate the studies, giving ample opportunities to the researchers to design experiments, elucidate drug targets, mechanisms and risk before testing them invitro or in-vivo. Computational interpretation or simulation of drug candidates or molecular compounds and demonstrating their binding analysis in the active region of target proteins opens pharmaceutical opportunities and the chance for the research laboratories to test the efficacy of drugs [344]. In this project, as shown in Chapter 2, WA is capable in decreasing the toxic effects of secreted $A \beta 40$. Upon establishing that WA has neuroprotective properties, we wanted to elucidate the mechanism underlying behind the neuroprotective action of WA. Therefore, we were interested in molecular simulation studies to support our hypothesis of WA interaction with $A \beta$ structure, resulting in morphological changes in the protein aggregates, which may prevent the further insoluble aggregation of $A \beta$ into plaques. In our in-silico approach, we have tested WA against $A D$ pathogenesis and after employing multiple online drug analysis computational tools and servers to predict the efficacy of WA compound, we showed WA as a potent compound against $A \beta$ induced $A D$ pathogenesis.

In this chapter, we examined the structure of $A \beta$ and studied its interaction with the neuroprotectant WA. There are multiple forms of amyloid, from single $A \beta$ peptide to interacting monomers, which further aggregate into multimers, protofibrils and then into mature fibrils. These mature fibrils aggregate with the help of seed oligomers, into insoluble aggregates of $A \beta$ plaques. Our drug candidate WA targets on $A \beta$ by two possible mechanisms. First, it interacts with single fibers and does not allow the fibrils to mature 
retaining their morphology and secondly it inhibits the aggregation, thereby reducing or inhibiting plaque formation (Figure 5.11). These results are important as therapeutic strategies as they show promising towards targeting amyloidogenesis has been associated with multiple and extensive range of neurological diseases. In AD specifically, abnormally misfolded amyloid is escaping immune clearance and their deposits in cells leads to conditions like memory loss, dementia and motor deficits [345].

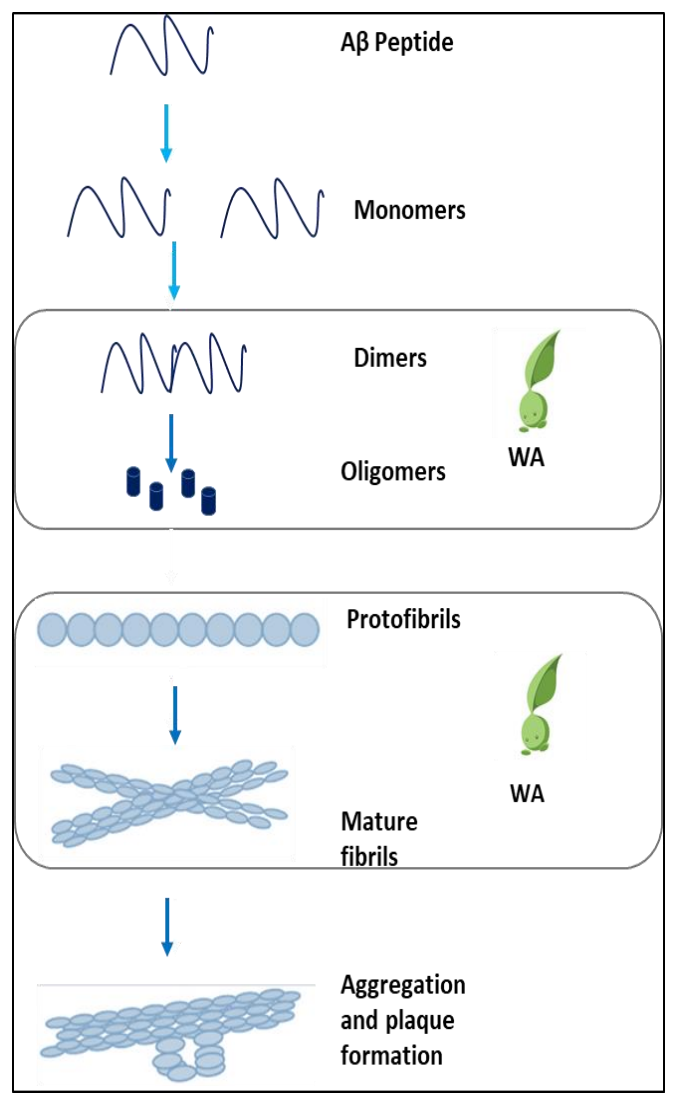

Figure 5.11 Working hypothesis of mechanism of WA

Our molecular simulation studied demonstrate that WA perfectly binds with the middle region of $A \beta 42$ protein by forming 3 distinct and stable hydrogen bonds. The mid region of $A \beta 42$ protein shows interaction and formation of hydrogen bonds between WA structure 
and specific amino acids ASN17, GLY15 and SER16 of the Aß42 protein. Our preliminary computational study on WA binding to $A \beta$ fibril has shown that WA binds in two physiologically relevant sites on $A \beta$ which are loop segment of the protein and the fibril interface, which affects both fibril growth and fibril stability. $A \beta$ is one of the important player leading to plaque formation is of important therapeutic concern and WA binds to the hydrophobic motif of amyloid beta and the binding is specific and stable, and is one step towards AD therapeutics. Many further studies are definitely required in this field to explain dynamic modelling interactions, stable environment, aggregated fibrillary toxicity, water coefficient, angle of the Hydrogen bonds and the bond strength. It is also important to run simulation for longer durations to ensure the stability of WA molecules in the amyloid complex, to avoid re-integration of the aggregates in the diseased scenario.

Our future in-silico studies will be focused on studying interaction of $\beta$-secretases with WA, to analyze if $\beta$-secretases can be potent therapeutic target for AD treatment or not. A variety of BACE1 inhibitors with promising properties have been identified with structurebased drug design strategies, leading to clinical development of selected inhibitors. Despite the clinical potential exhibited by several inhibitors, an effective FDA approved BACE1 inhibitor is still lacking. Therefore, we will investigate the role of WA as a potential BACE1 inhibitor. Several x-ray crystal structures of BACE1 with or without inhibitors are available (e.g. pdb id: 1FKN, 3DM6, 3KYR, 2G94, 2VKM, 1XS7), showing slight conformational variability in BACE1-compound complexes. Therefore, we will generate diverse array of conformations by performing $>100$ ns MD simulations of BACE1 and sample about 1,000 conformations for docking/screening with WA. About top five WAbound complexes will be selected for performing MD simulations to examine the stability of the complex. Due to the similarity of the active site binding pockets in BACE1 and 
BACE2, selectivity of the BACE1 inhibitor can be limited. To differentiate the binding of WA between BACE1 and BACE2, we will perform similar screening analysis of WA against conformations of BACE2. The top-scoring binding poses that are different in WA-BACE1 compared to WA-BACE2 will be selected for further MD simulations. 


\section{CHAPTER 6: FINAL CONCLUSIONS AND FUTURE DIRECTIONS}

Parts of this chapter appear in the journal Biosensors \& Bioelectronics. Published online 2016 Jun15. doi: 10.1016/j.bios.2016.01.065.

$A D$ is described as the progressive and irreversible neurodegenerative disorder, characterized by the presence of abnormal extracellular and insoluble $A \beta$ peptide ([346348] which aggregate into neuritic plaques [349-351]. A $\beta$ protein deposition in the human brain is one of the key component in possible theories of the AD pathogenesis [292]. The presence of hyperphosphorylated tau protein in the brain resulting in the formation of NFTs is reported $[352,353]$. Other theories propose the role of the APOE $\epsilon 4$ allele [354] which may pose as a risk factor in AD pathogenesis $[355, \underline{356}]$. The continuous accumulation of the plaques and tangles in the brain result into toxicity leading to cognitive impairments [357]. A $\beta$ is produced from a transmembrane APP through abnormal cleavage by the $\beta$ and $y$ secretases $[14, \underline{318}] . A \beta$ proteins can occur in soluble and fibrillary forms, and the senile plaque which are typical in $A D$ pathogenesis are aberrant plaques which are dense and insoluble comprised of $A \beta$ fibrils and toxic oligomers [358]. Furthermore, $A \beta$ production triggers abnormal Tau processing, linking $A \beta$ accumulation and tau protein phosphorylation [359] which are also believed to be an important contributor in $A D$ pathogenesis. NFTS are constitutive of Tau which is a microtubule-associated protein and in diseased scenario Tau phosphorylates and leads to distortion in the microtubular conformations, implicating towards in the pathological progression of $A D$ [360]. There are varied opinions towards the pathological features behind $A D$ mechanism, and amongst them $A \beta$ hypothesis is the most accepted one, associated with the onset and progression of $A D . A B$ is found in two main isoforms (amino acid chain length are different); namely $A \beta 40$ and $A \beta 42[\underline{361}, \underline{362}]$ where $A \beta 42$ is more toxic but less abundant compared to $A \beta 40$ 
which is reported in high concentrations in the AD brain. These peptides undergo self and spontaneous association leading to supramolecular aggregated assemblies of dense and insoluble. Plaque formation leads to deteriorated neuronal synapses and communication, therefore producing more neurotoxicity. The $A \beta$ aggregation leads to release of ROS and $\mathrm{NO}$, contributing to neurotoxicity, altering immunological responses, boosting abnormal secretion of pro-inflammatory cytokines, formation of soluble neurotoxic oligomers and aggregating fibrils leading to severe neuropathological conditions, contributing towards poor neuronal communications, memory deficits, motor disorientations and dementia. The $A \beta$ oligomers attach with the neuronal lipid bilayer and induce abnormal alterations, depolarization, Ca ion up-regulation and increase in ROS levels [363]. In our future studies, we intend to study the effect of WA on the Ca ions regulation and function which will give us better insight into WA action mechanism as these $A \beta$ associated neurodegenerative pathological changes damage the neuronal network by altering synaptic structure and functionality resulting in the spatial memory weakening linked with neuronal dysfunctions.

We also want to study the effect of WA on the mitochondrial dysfunctions caused by $A \beta$ load as a result of altered energy homeostasis and insulin signaling pathways. Most deteriorating $A \beta$ neurotoxic mechanisms comprise oxidative stress and mitochondrial damage. It has been reported that the perivascular accumulation of $A \beta$ harms the $B B B$, including microbleedings, damaged neuronal network, and abnormal inflammatory reactions [364]. Therefore, in our future studies we want to study WA as the endothelial protective drug by lowering oxidative damage and vascular dysfunction, favoring healthy BBB. 
As a response to abnormal concentrations of $A \beta$ plaques and NFTs aggregation, microglial infiltration at the site has been reported which plays a vital role in triggering innate immune response against the aggregation. Additionally, the increase in release of inflammatory cytokines attracts microglia towards the plaque-populated regions, therefore worsening the situation and contributing towards intense neuro-inflammation [365-369]. The link between microglia and AD is well studied and it has been reported that microglia tend to accumulate near the senile plaques in the $A D$ brain [370]. $A \beta$ aggregation induce microglial infiltration and induce neuroinflammation by binding with microglial innate immune receptors (G-Protein-Coupled Receptors), which initiate an inflammatory cascade [371]. In AD, synaptic impairment and associated neuronal death prompted by $A \beta$ peptide are in part due to microglial and astrocyte cells activation. Microglial activation results in pro-inflammatory cytokines and ROS production towards chronic inflammatory process. Additionally, astrocytes are involved with neuroinflammation, too, and are activated by A $\beta$ load, pathogens, and oxidative stress, producing inflammatory cytokines cyclooxygenase2 enzyme, augmenting $A \beta$ production $[\underline{366}, \underline{372}, \underline{373}]$.

Several studies indicate towards some correlations of $A \beta$ to decreased memory scores $[\underline{374}, \underline{375}]$-and difficulty of patients to perform memory tasks $[\underline{293}, \underline{376}$. Therefore, in our understanding it becomes utmost important to study the effect of drugs which can target $A \beta$ without causing cytotoxicity in neuronal cells. Therefore, in this study we introduced WA, and explored its potential in decreasing $A \beta$. WA is a purified steroidal lactone, derived from the parent component Ashwagandha (from W. Somnifera). Our previous study has shown that the most evident and abundant component of $\mathrm{ASH}$ are Withanolides, and WA is an active moiety of Withanolides with significant $A \beta$ decreasing properties [119]. 
In the current study, we demonstrated the neuroprotective role of WA against $A \beta 40$ toxicity. We have also shown that $A \beta 40$ associated toxicity is boosted by the presence of HIV-1 Tat and drug of abuse Cocaine in-vitro. We have shown that WA is able to reduce the A A $\beta$ levels induced by HIV-1 Tat and Cocaine. WA reduces associated neurotoxicity and boosts synaptic plasticity and neuronal communications, by promoting synaptic plasticity associated gene expression and aiding in dendritic length and communication in cultured neurons, which we have discussed in Chapter 2.

In case of amyloidogenic diseased pathway the APP protein is abnormally cleaved by $\mathrm{Y}$ and $\beta$ secretases leading to the production of $A \beta$ peptides [247], which then aggregate and form insoluble plaques $[\underline{35}, \underline{377-379}]$. Therefore, we wanted to look at the mechanism by which WA could be working. Upon performing $y$ and $\beta$ secretases targeting ELISAs, from the WA treated cell supernatants, we found that WA showed no effect on the levels of $y$ secretases. Additionally, we also wanted to look at the effect of WA on the $\beta$ secretases, but we were not able to detect differences in $\beta$ secretases by the in-vitro ELISA studies, which may be due to undetectable amount of $\beta$ secretases in the SHAPP in-vitro cell cultures. This definitely requires future in-vivo studies where we can work with APP/PS1 mice models to learn more about the effect of WA on $\beta$ secretases, if any. Even though, cohesive studies including systematic $A \beta$ clearance by targeting mechanism and related molecules are scarce, we believe that $\mathrm{y}$ and $\beta$ secretases may be potential drug targets. We believe that targeting $A D$ should not only be approached by targeting $A \beta$ only in CNS, but targeting signaling enzymes and factors may be impotent therapeutic approach as well. 
In continuation to the effect of WA on $A \beta$, we also wanted to venture in the field of $A \beta$ associated inflammation, and effect of W on inflammation, if any. As discussed in Chapter 3 , inflammation is one of the associated atrocities during $A \beta$ associated neurotoxicity, and there is a need of drug or combination of drugs to target inflammation. In order to address the inflammation due to $A \beta$ accumulation, we have shown that WA inhibits NF-kB associated neuroinflammation, as Our NF-KB assay showed that WA was able to inhibit the expression of NF-kB2 and down-regulated RELA transcription factors, which plays a major role in the expression of inflammatory chemokines and cytokines. Our second drug of interest CRID3 was shown to downregulate various chemokines like C5, CCL20, CCL26, CCL5 and receptors like CCR6 and CXCR2. Additionally, CRID3 targets IL1- $\beta$ by reducing the levels of Caspase-1 enzyme, which converts pro- IL1- $\beta$ to active e IL1- $\beta$. These results are very important to target inflammation during $A \beta$ neurotoxicity and microglial infiltration. In summary, our study highlights the mechanism by which WA modifies and inhibits NF-KB activity. We also show the role of CRID3 in targeting inflammasome activation as it targets IL-1 $\beta$ and down-regulation of IL-1 $\beta$ could be of potential importance in targeting inflammasome activation. Future studies can focus more on studying the effect of WA and CRID3 individually at major cytokines associated with inflammation at gene and protein expression levels. This will give us a clear insight on the targets of WA and CRID3 in inflammation, which could be of immense therapeutic potential against $A \beta$ induced inflammation in $A D$ or $A D$ - like diseases.

Having learnt the importance of WA against $A \beta$ induced neuronal toxicity and antiinflammatory role, the next question that we needed to address was the availability of hydrophobic WA across the BBB into the CNS. The BBB is a structural and functional obstruction between the CNS and the peripheral, it maintains the CNS milieu 
that is necessary for neural function. It consists of network of endothelial cells, pericytes, and astrocytes packed together forming Tight Junctions, which regulates minimal transmigration of molecules from the blood side to the brain across the BBB [요이. The major issue in targeting drugs to CNS is the presence of BBB, therefore it is utmost important to tackle BBB transmigration, without distorting the stability and functionality of BBB and increasing the drug bioavailability on to the CNS. To overcome the transmigration shortcoming, nanotechnology has been a promising candidate. Utilizing the role of nanoparticles as efficient drug carriers, we have utilized liposomes as drug carrier here as liposomes have an advantage over other nanoparticles. They can be loaded with both hydrophobic and hydrophilic drugs, because the lipid bilayer of the liposomes captures the hydrophobic drug molecules, which aid in delivering hydrophobic drugs efficiently and targeting specific regions in the brain for different CNS disease treatments. We modelled the 3D in-vitro BBB model utilizing primary human endothelial cells, astrocytes and pericytes grown on a trans-well insert with a membrane, which mimics the

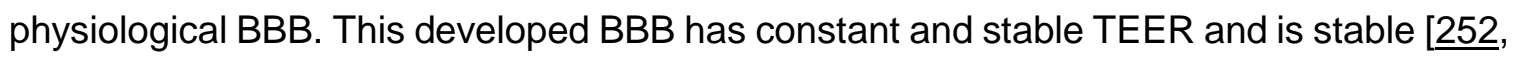
381]. In Chapter 4 we have shown the development of the WA-loaded liposomes (WALNF), and characterized them for their size and drug binding and transmigration efficiency. We report that developed WA-LNF had a hydrodynamic size of $\sim 450 \mathrm{~nm}$ in size and showed $28 \%$ drug binding capacity, with no cellular toxicity. We also studied the BBB integrity by measuring the trans-endothelial electrical resistance (TEER) and paracellular permeability using fluorescein isothiocyanate (FITC)-dextran transmigration of the developed NF and reported that WA-LNF showed almost $50 \%$ transmigration and did not disrupt the integrity of the 3D in-vitro BBB. 
Finally, we wanted to elucidate the basic mechanism of WA's interaction with the $A \beta$ protein. $A \beta$ monomer comprises of $40-42$ amino acid depending on the isoforms of amyloid. We were interested in demonstrating the structural conformation of the $A \beta$ protein, and wanted to study if WA binds or interacts with amyloid protein, and if yes, then where exactly and with what stability. Our molecular simulation studies demonstrate that WA perfectly binds with the middle region of $A \beta 42$ protein by forming 3 distinct and stable hydrogen bonds. The mid region of ab42 protein shows ionic interaction and formation of hydrogen bonds between WA constituents and specific amino acids ASN17, GLY15 and SER16 of the $A \beta 42$ protein. Our preliminary computational study on WA binding to $A \beta$ fibril has shown that WA binds in two physiologically relevant sites on $A \beta$, which are loop segment of the protein and the fibril interface, which affects both fibril growth and fibril stability. Future directions in continuation to this study is Amyloid protein crystal structure assessment via NMR and Mass spectrometry. Crystallization of pure protein derived from the brain lysates of animal models, and studying them via Nuclear Magnetic Resonance studies depicting the structure and binding potential of the protein with the WA drug candidate will give us more clear understanding of the mechanism of action of WA. We also want to look at the effect of WA on various isoforms of the Amyloid, as at clinical level, $\mathrm{AD}$ brains have multiple isoforms of amyloids reported.

There are many options for future studies to advance the work presented here [382]. The entire work presented here is in-vitro as this is preliminary work in this field. Major advanced studies at in-vivo level are required with respect to study the role of WA against Alzheimer's disease. Our focus in future is to design a magneto-liposomal Nanoformulation towards increasing the efficacy of NFs to higher percentage of drug loading within the liposome (without increasing overall size of NF), and optimization of 
magnetic treatment (time v/s field strength) for better and longer therapeutic efficacy. We plan to develop and characterize magneto electric liposomal containing multiple drugs including WA (anti-amyloid and anti-inflammation), CRID3 (anti-inflammation) \& BD1063 (anti-cocaine) and study the cargo's non-invasive BBB transmigration, controlled release and therapeutic efficacy in the animal models.

In future, we plan to study the therapeutic efficacy, cytotoxicity and on-demand controlled release of nanoformulations in a cocaine-injected double transgenic (APP/PS1 and Tat) HAND mouse model. APP/PS1 and Tat double transgenic mouse model exhibit remarkable elevation of $A \beta$ production associated with certain behavioral abnormalities as well as HAND pathology in the brain. The objective of this study will be to validate the outcome of the pre-screening studies using HAND mouse model and to evaluate the transmigration of the NFs across the BBB under the influence of an external magnetic field, on demand controlled release on a.c. magnetic stimulation and study the efficacy of the developed NFs in APP/PS1- Tat mouse model. We will study therapeutic efficacy, toxicity and in vivo efficacy of the developed NF and will perform immunohistochemistry experiments to study $A \beta$ deposition. In addition, we will analyze neurobehavioral aspects in NF injected animals including changes in locomotor activity, motor strength, ability, balance, and coordination skills, fear-motivated avoidance, novel object recognition, spatial learning and memory ability, compared to untreated control mice models by utilizing neurobehavioral tests like Locomotor Sensitization, Rotarod Test, Fear Conditioning/Active Avoidance, novel object recognition test and Morris Water Maze. These experiments will provide us basic understanding about the efficacy of the individual drugs as well as the NF cargo, and drug targeting, delivery and sustained release. Our developed nanoformulation will be a potent therapeutic cargo towards combating AD and 
AD-like diseases. We believe that successful delivery of our NF will lead to the inhibition of Tat and cocaine induced $A \beta$ and associated neuro-inflammation that will result in the improved neuro-cognitive functions in HIV-infected cocaine abusing and aging patients.

\section{REFERENCES}

1. Adav, S.S. and S.K. Sze, Insight of brain degenerative protein modifications in the pathology of neurodegeneration and dementia by proteomic profiling. Molecular brain, 2016. 9(1): p. 92.

2. Leandro, P. and C.M. Gomes, Protein misfolding in conformational disorders: rescue of folding defects and chemical chaperoning. Mini reviews in medicinal chemistry, 2008. 8(9): p. 901-911.

3. Tran, L. and T. Ha-Duong, Exploring the Alzheimer amyloid- $\beta$ peptide conformational ensemble: A review of molecular dynamics approaches. Peptides, 2015. 69: p. 86-91.

4. Horwich, A., Protein aggregation in disease: a role for folding intermediates forming specific multimeric interactions. The Journal of clinical investigation, 2002. 110(9): p. 1221-1232.

5. Selkoe, D.J., Cell biology of protein misfolding: the examples of Alzheimer's and Parkinson's diseases. Nature cell biology, 2004. 6(11): p. 1054.

6. Blessed, G., B.E. Tomlinson, and M. Roth, The association between quantitative measures of dementia and of senile change in the cerebral grey matter of elderly subjects. The British Journal of Psychiatry, 1968. 114(512): p. 797-811.

7. O'Brien, R.J. and P.C. Wong, Amyloid precursor protein processing and Alzheimer's disease. Annual review of neuroscience, 2011. 34: p. 185-204.

8. Henry, W., H. Querfurth, and F. LaFerla, Mechanisms of disease Alzheimer's disease. New Engl J Med, 2010. 362: p. 329-44.

9. Alzheimer's, A., 2015 Alzheimer's disease facts and figures. Alzheimer's \& dementia: the journal of the Alzheimer's Association, 2015. 11(3): p. 332.

10. Goedert, M., Alzheimer's and Parkinson's diseases: The prion concept in relation to assembled $A \beta$, tau, and $\alpha$-synuclein. Science, 2015. 349(6248): p. 1255555.

11. Chen, J.X. and S.S. Yan, Role of mitochondrial amyloid- $\beta$ in Alzheimer's disease. Journal of Alzheimer's Disease, 2010. 20(s2): p. S569-S578.

12. Crews, L. and E. Masliah, Molecular mechanisms of neurodegeneration in Alzheimer's disease. Human molecular genetics, 2010. 19(R1): p. R12-R20. 
13. Oh, E.S., et al., Amyloid precursor protein increases cortical neuron size in transgenic mice. Neurobiology of aging, 2009. 30(8): p. 1238-1244.

14. Thinakaran, G. and E.H. Koo, Amyloid precursor protein trafficking, processing, and function. Journal of Biological Chemistry, 2008. 283(44): p. 29615-29619.

15. Young-Pearse, T.L., et al., A critical function for $\beta$-amyloid precursor protein in neuronal migration revealed by in utero RNA interference. Journal of Neuroscience, 2007. 27(52): p. 14459-14469.

16. Meziane, H., et al., Memory-enhancing effects of secreted forms of the $\beta$-amyloid precursor protein in normal and amnestic mice. Proceedings of the National Academy of Sciences, 1998. 95(21): p. 12683-12688.

17. Selkoe, D.J., Cell biology of the amyloid beta-protein precursor and the mechanism of Alzheimer's disease. Annual review of cell biology, 1994. 10(1): p. 373-403.

18. Hefter, D., et al., Amyloid precursor protein protects neuronal network function after hypoxia via control of voltage-gated calcium channels. Journal of Neuroscience, 2016. 36(32): p. 8356-8371.

19. Shoji, M., et al., Production of the Alzheimer amyloid beta protein by normal proteolytic processing. Science, 1992. 258(5079): p. 126-129.

20. Kimberly, W.T., et al., The intracellular domain of the B-amyloid precursor protein is stabilized by Fe65 and translocates to the nucleus in a notch-like manner. Journal of Biological Chemistry, 2001.

21. Bergmans, B.A. and B. De Strooper, $y$-secretases: from cell biology to therapeutic strategies. The Lancet Neurology, 2010. 9(2): p. 215-226.

22. Tu, S., et al., Oligomeric $A \beta$-induced synaptic dysfunction in Alzheimer's disease. Molecular neurodegeneration, 2014. 9(1): p. 48.

23. Claeysen, S., et al., Alzheimer culprits: cellular crossroads and interplay. Cellular signalling, 2012. 24(9): p. 1831-1840.

24. Marcus, J.N. and J. Schachter, Targeting post-translational modifications on tau as a therapeutic strategy for Alzheimer's disease. Journal of neurogenetics, 2011. 25(4): p. 127-133.

25. Lee, V.M., M. Goedert, and J.Q. Trojanowski, Neurodegenerative tauopathies. Annual review of neuroscience, 2001. 24(1): p. 1121-1159.

26. Nizynski, B., W. Dzwolak, and K. Nieznanski, Amyloidogenesis of Tau protein. Protein Science, 2017. 26(11): p. 2126-2150.

27. De Strooper, B. and J. Woodgett, Alzheimer's disease: Mental plaque removal. Nature, 2003. 423(6938): p. 392. 
28. Phiel, C.J., et al., GSK-3a regulates production of Alzheimer's disease amyloid- $\beta$ peptides. Nature, 2003. 423(6938): p. 435.

29. Hernández, F. and J. Avila, The role of glycogen synthase kinase 3 in the early stages of Alzheimers' disease. FEBS letters, 2008. 582(28): p. 3848-3854.

30. Bossy-Wetzel, E., R. Schwarzenbacher, and S.A. Lipton, Molecular pathways to neurodegeneration. Nature medicine, 2004. 10(7): p. S2.

31. Cho, J.-H. and G.V. Johnson, Glycogen synthase kinase $3 \beta$ induces caspasecleaved tau aggregation in situ. Journal of Biological Chemistry, 2004. 279(52): p. 54716-54723.

32. Kuruva, C.S. and P.H. Reddy, Amyloid beta modulators and neuroprotection in Alzheimer's disease: a critical appraisal. Drug discovery today, 2017. 22(2): p. 223233.

33. Nizzari, M., et al., Neurodegeneration in Alzheimer disease: role of amyloid precursor protein and presenilin 1 intracellular signaling. Journal of Toxicology, 2012. 2012.

34. Dries, D.R. and G. Yu, Assembly, maturation, and trafficking of the $y$-secretase complex in Alzheimer's disease. Current Alzheimer Research, 2008. 5(2): p. 132146.

35. Edbauer, D., et al., Reconstitution of $\mathrm{Y}$-secretase activity. Nature cell biology, 2003. 5(5): p. 486.

36. Shen, J. and R.J. Kelleher, The presenilin hypothesis of Alzheimer's disease: evidence for a loss-of-function pathogenic mechanism. Proceedings of the National Academy of Sciences, 2007. 104(2): p. 403-409.

37. Waddington, C.H., The epigenotype. International journal of epidemiology, 2011. 41(1): p. 10-13.

38. Lardenoije, R., et al., The epigenetics of aging and neurodegeneration. Progress in neurobiology, 2015. 131: p. 21-64.

39. Sweatt, J.D., The emerging field of neuroepigenetics. Neuron, 2013. 80(3): p. 624632.

40. Tsankova, N., et al., Epigenetic regulation in psychiatric disorders. Nature Reviews Neuroscience, 2007. 8(5): p. 355.

41. Day, J.J. and J.D. Sweatt, DNA methylation and memory formation. Nature neuroscience, 2010. 13(11): p. 1319. 
42. Landgrave-Gómez, J., O. Mercado-Gómez, and R. Guevara-Guzmán, Epigenetic mechanisms in neurological and neurodegenerative diseases. Frontiers in cellular neuroscience, 2015. 9: p. 58.

43. Sezgin, Z. and Y. Dincer, Alzheimer's disease and epigenetic diet. Neurochemistry international, 2014. 78: p. 105-116.

44. Balazs, R., Epigenetic mechanisms in Alzheimer's disease. Degenerative Neurological and Neuromuscular Disease, 2014. 4: p. 85-102.

45. Hernandez, D.G., et al., Distinct DNA methylation changes highly correlated with chronological age in the human brain. Human molecular genetics, 2011. 20(6): p. 1164-1172.

46. Mastroeni, D., et al., Epigenetic mechanisms in Alzheimer's disease. Neurobiology of aging, 2011. 32(7): p. 1161-1180.

47. Francis, Y.I., et al., Dysregulation of histone acetylation in the APP/PS1 mouse model of Alzheimer's disease. Journal of Alzheimer's Disease, 2009. 18(1): p. 131139.

48. Delgado-Morales, R., et al., Epigenetic mechanisms during ageing and neurogenesis as novel therapeutic avenues in human brain disorders. Clinical epigenetics, 2017. 9(1): p. 67.

49. Ji, K., et al., Microglia actively regulate the number of functional synapses. PloS one, 2013. 8(2): p. e56293.

50. Heneka, M.T., et al., Neuroinflammation in Alzheimer's disease. The Lancet Neurology, 2015. 14(4): p. 388-405.

51. Bamberger, M.E., et al., A cell surface receptor complex for fibrillar $\beta$-amyloid mediates microglial activation. Journal of Neuroscience, 2003. 23(7): p. 26652674 .

52. Liu, Y., et al., LPS receptor (CD14): a receptor for phagocytosis of Alzheimer's amyloid peptide. Brain, 2005. 128(8): p. 1778-1789.

53. Wilkinson, K. and J. El Khoury, Microglial scavenger receptors and their roles in the pathogenesis of Alzheimer's disease. International journal of Alzheimer's disease, 2012. 2012.

54. Malito, E., R.E. Hulse, and W.-J. Tang, Amyloid $\beta$-degrading cryptidases: insulin degrading enzyme, presequence peptidase, and neprilysin. Cellular and Molecular Life Sciences, 2008. 65(16): p. 2574-2585.

55. Hickman, S.E., E.K. Allison, and J. El Khoury, Microglial dysfunction and defective $\beta$-amyloid clearance pathways in aging Alzheimer's disease mice. Journal of Neuroscience, 2008. 28(33): p. 8354-8360. 
56. Wang, Y., et al., TREM2 lipid sensing sustains the microglial response in an Alzheimer's disease model. Cell, 2015. 160(6): p. 1061-1071.

57. Hsieh, C.L., et al., A role for TREM2 ligands in the phagocytosis of apoptotic neuronal cells by microglia. Journal of neurochemistry, 2009. 109(4): p. 11441156.

58. Lannes, N., et al., Microglia at center stage: a comprehensive review about the versatile and unique residential macrophages of the central nervous system. Oncotarget, 2017. 8(69): p. 114393.

59. Datta, M., et al., Histone deacetylases 1 and 2 regulate microglia function during development, homeostasis, and neurodegeneration in a context-dependent manner. Immunity, 2018. 48(3): p. 514-529. e6.

60. Giunti, D., et al., Can we switch microglia's phenotype to foster neuroprotection? Focus on multiple sclerosis. Immunology, 2014. 141(3): p. 328-339.

61. Salter, M.W. and B. Stevens, Microglia emerge as central players in brain disease. Nature medicine, 2017. 23(9): p. 1018.

62. Dukhinova, M., E. Kopeikina, and E.D. Ponomarev, Usage of Multiparameter Flow Cytometry to Study Microglia and Macrophage Heterogeneity in the Central Nervous System During Neuroinflammation and Neurodegeneration, in Cellular Heterogeneity. 2018, Springer. p. 167-177.

63. McHugh, D., et al., N-arachidonoyl glycine, an abundant endogenous lipid, potently drives directed cellular migration through GPR18, the putative abnormal cannabidiol receptor. BMC neuroscience, 2010. 11(1): p. 44.

64. Serrano-Pozo, A., et al., Differential relationships of reactive astrocytes and microglia to fibrillar amyloid deposits in Alzheimer disease. Journal of Neuropathology \& Experimental Neurology, 2013. 72(6): p. 462-471.

65. Takata, K., et al., Possible involvement of small oligomers of amyloid- $\beta$ peptides in 15-deoxy- $\Delta 12,14$ prostaglandin J2-sensitive microGlial activation. Journal of pharmacological sciences, 2003. 91(4): p. 330-333.

66. Lindberg, C., et al., $\beta$-amyloid protein structure determines the nature of cytokine release from rat microglia. Journal of molecular neuroscience, 2005. 27(1): p. 112.

67. Wilcock, D.M., et al., Intracranially administered anti-A $\beta$ antibodies reduce $\beta$ amyloid deposition by mechanisms both independent of and associated with microglial activation. Journal of Neuroscience, 2003. 23(9): p. 3745-3751.

68. Peng, L., et al., The chemerin receptor CMKLR1 is a functional receptor for amyloid- $\beta$ peptide. Journal of Alzheimer's Disease, 2015. 43(1): p. 227-242. 
69. Kim, B., et al., Uridine 5'-diphosphate induces chemokine expression in microglia and astrocytes through activation of the P2Y6 receptor. The Journal of Immunology, 2011: p. 1000212.

70. Valcour, V.G., et al., Cognitive impairment in older HIV-1-seropositive individuals: prevalence and potential mechanisms. AIDS (London, England), 2004. 18(Suppl 1): p. S79.

71. Becker, J.T., et al., Prevalence of cognitive disorders differs as a function of age in HIV virus infection. Aids, 2004. 18: p. 11-18.

72. Esiri, M.M., S.C. Biddolph, and C.S. Morris, Prevalence of Alzheimer plaques in AIDS. Journal of Neurology, Neurosurgery \& Psychiatry, 1998. 65(1): p. 29-33.

73. Green, D.A., et al., Brain deposition of beta-amyloid is a common pathologic feature in HIV positive patients. Aids, 2005. 19(4): p. 407-411.

74. Pulliam, L., HIV regulation of amyloid beta production. Journal of Neuroimmune Pharmacology, 2009. 4(2): p. 213-217.

75. Scannevin, R.H., Therapeutic strategies for targeting neurodegenerative protein misfolding disorders. Current opinion in chemical biology, 2018. 44: p. 66-74.

76. Cummings, J., et al., Alzheimer's disease drug development pipeline: 2017. Alzheimer's \& Dementia: Translational Research \& Clinical Interventions, 2017. 3(3): p. 367-384.

77. Simmons, D., et al., Neuroprotective Strategies for Alzheimer's Disease Prevention and Therapy, in Developing Therapeutics for Alzheimer's Disease. 2016, Elsevier. p. 437-458.

78. Wadghiri, Y.Z., et al., Detection of Alzheimer's amyloid in transgenic mice using magnetic resonance microimaging. Magnetic Resonance in Medicine: An Official Journal of the International Society for Magnetic Resonance in Medicine, 2003. 50(2): p. 293-302.

79. Skaat, $\mathrm{H}$. and S. Margel, Synthesis of fluorescent-maghemite nanoparticles as multimodal imaging agents for amyloid- $\beta$ fibrils detection and removal by a magnetic field. Biochemical and biophysical research communications, 2009. 386(4): p. 645-649.

80. Skaat, H., et al., Effect of maghemite nanoparticles on insulin amyloid fibril formation: selective labeling, kinetics, and fibril removal by a magnetic field. Journal of Biomedical Materials Research Part A: An Official Journal of The Society for Biomaterials, The Japanese Society for Biomaterials, and The Australian Society for Biomaterials and the Korean Society for Biomaterials, 2009. 91(2): p. 342-351. 
81. Siegemund, T., et al., Thioflavins released from nanoparticles target fibrillar amyloid $\beta$ in the hippocampus of APP/PS1 transgenic mice. International journal of developmental neuroscience, 2006. 24(2-3): p. 195-201.

82. Henley, D.B., et al., Development of semagacestat (LY450139), a functional Ysecretase inhibitor, for the treatment of Alzheimer's disease. Expert opinion on pharmacotherapy, 2009. 10(10): p. 1657-1664.

83. Choi, J.-s., et al., Nanoparticle assisted magnetic resonance imaging of the early reversible stages of amyloid $\beta$ self-assembly. Chemical Communications, 2008(19): p. 2197-2199.

84. Dubertret, B., et al., In vivo imaging of quantum dots encapsulated in phospholipid micelles. Science, 2002. 298(5599): p. 1759-1762.

85. Georganopoulou, D.G., et al., Nanoparticle-based detection in cerebral spinal fluid of a soluble pathogenic biomarker for Alzheimer's disease. Proceedings of the National Academy of Sciences, 2005. 102(7): p. 2273-2276.

86. Kolb, H.C., M. Finn, and K.B. Sharpless, Click chemistry: diverse chemical function from a few good reactions. Angewandte Chemie International Edition, 2001. 40(11): p. 2004-2021.

87. Kang, D.-Y., et al., Ultra-sensitive immunosensor for $\beta$-amyloid (1-42) using scanning tunneling microscopy-based electrical detection. Biosensors and Bioelectronics, 2009. 24(5): p. 1431-1436.

88. Tiwari, S., et al., Withaferin A suppresses Beta amyloid in APP expressing cells: Studies for Tat and Cocaine associated neurological dysfunctions. Frontiers in aging neuroscience, 2018. 10: p. 291.

89. Hsiao, I.L., et al., Effects of silver nanoparticles on the interactions of neuron-and glia-like cells: Toxicity, uptake mechanisms, and lysosomal tracking. Environmental toxicology, 2017. 32(6): p. 1742-1753.

90. Hsiao, I.-L., et al., Trojan-horse mechanism in the cellular uptake of silver nanoparticles verified by direct intra-and extracellular silver speciation analysis. Environmental science \& technology, 2015. 49(6): p. 3813-3821.

91. Leszek, J., et al., Nanotechnology for Alzheimer disease. Current Alzheimer Research, 2017. 14(11): p. 1182-1189.

92. Fernandes, J., et al., Amino acid conjugated chitosan nanoparticles for the brain targeting of a model dipeptidyl peptidase-4 inhibitor. International journal of pharmaceutics, 2018.

93. Glenner, G.G. and C.W. Wong, Alzheimer's disease: initial report of the purification and characterization of a novel cerebrovascular amyloid protein. Biochemical and biophysical research communications, 1984. 120(3): p. 885-890. 
94. Glenner, G. and C. Wong, Alzheimer's disease: initial report of the purification and characterization of a novel cerebrovascular amyloid protein. Alzheimer Disease \& Associated Disorders, 1988. 2(2): p. 134.

95. Glenner, G.G. and C.W. Wong, Alzheimer's disease and Down's syndrome: sharing of a unique cerebrovascular amyloid fibril protein. Biochemical and biophysical research communications, 1984. 122(3): p. 1131-1135.

96. Masters, C.L., et al., Amyloid plaque core protein in Alzheimer disease and Down syndrome. Proceedings of the National Academy of Sciences, 1985. 82(12): p. 4245-4249.

97. Kang, J., et al., The precursor of Alzheimer's disease amyloid A4 protein resembles a cell-surface receptor. Nature, 1987. 325(6106): p. 733.

98. Goldgaber, D., et al., Characterization and chromosomal localization of a cDNA encoding brain amyloid of Alzheimer's disease. Science, 1987. 235(4791): p. 877880.

99. Tanzi, R.E., et al., Amyloid beta protein gene: cDNA, mRNA distribution, and genetic linkage near the Alzheimer locus. Science, 1987. 235(4791): p. 880-884.

100. Olson, M.I. and C.-M. Shaw, Presenile dementia and Alzheimer's disease in mongolism. Brain, 1969. 92(1): p. 147-156.

101. Levy, E., et al., Mutation of the Alzheimer's disease amyloid gene in hereditary cerebral hemorrhage, Dutch type. Science, 1990. 248(4959): p. 1124-1126.

102. Van Broeckhoven, C., et al., Amyloid beta protein precursor gene and hereditary cerebral hemorrhage with amyloidosis (Dutch). Science, 1990. 248(4959): p. 1120-1122.

103. Goate, A., et al., Segregation of a missense mutation in the amyloid precursor protein gene with familial Alzheimer's disease. Nature, 1991. 349(6311): p. 704.

104. Mullan, M., et al., A pathogenic mutation for probable Alzheimer's disease in the APP gene at the $\mathrm{N}$-terminus of $\beta$-amyloid. Nature genetics, 1992. 1(5): p. 345.

105. Buckner, R.L., et al., Molecular, structural, and functional characterization of Alzheimer's disease: evidence for a relationship between default activity, amyloid, and memory. Journal of Neuroscience, 2005. 25(34): p. 7709-7717.

106. Hendriks, L., et al., Presenile dementia and cerebral haemorrhage linked to a mutation at codon 692 of the $\beta$-amyloid precursor protein gene. Nature genetics, 1992. 1(3): p. 218.

107. Association, A.s., 2014 Alzheimer's disease facts and figures. Alzheimer's \& Dementia, 2014. 10(2): p. e47-e92. 
108. Nair, M., et al., Externally controlled on-demand release of anti-HIV drug using magneto-electric nanoparticles as carriers. Nature communications, 2013. 4: p. 1707.

109. Nair, M., et al., Getting into the brain: potential of nanotechnology in the management of NeuroAIDS. Advanced drug delivery reviews, 2016. 103: p. 202217.

110. Kaushik, A., et al., Nano-biosensors to detect beta-amyloid for Alzheimer's disease management. Biosensors and bioelectronics, 2016. 80: p. 273-287.

111. Sagar, V., et al., Towards nanomedicines for neuroAIDS. Reviews in medical virology, 2014. 24(2): p. 103-124.

112. Oguntibeju, O.O., Quality of life of people living with HIV and AIDS and antiretroviral therapy. HIV/AIDS (Auckland, NZ), 2012. 4: p. 117.

113. UNAIDS, G., Global AIDS update 2016. Geneva, Switzerland: World Health Organization Library, 2016.

114. Saylor, D., et al., HIV-associated neurocognitive disorder-pathogenesis and prospects for treatment. Nature Reviews Neurology, 2016. 12(4): p. 234.

115. Sabin, C.A., Do people with HIV infection have a normal life expectancy in the era of combination antiretroviral therapy? BMC medicine, 2013. 11(1): p. 251.

116. Bredesen, D. and M. Rosenblum, Neurological manifestations of the acquired immunodeficiency syndrome (AIDS): experience at UCSF and review of the literature. J Neurosurg, 1985. 62: p. 475-95.

117. Koppel, B.S., et al., Central nervous system involvement in patients with acquired immune deficiency syndrome (AIDS). Acta neurologica scandinavica, 1985. 71(5): p. 337-353.

118. Dorszewska, J., et al., Molecular basis of familial and sporadic alzheimer's disease. Current Alzheimer Research, 2016. 13(9): p. 952-963.

119. Kurapati, K.R.V., et al., Ashwagandha (Withania somnifera) reverses $\beta$-amyloid142 induced toxicity in human neuronal cells: implications in HIV-associated neurocognitive disorders (HAND). PLoS One, 2013. 8(10): p. e77624.

120. Kurapati, K.R.V., et al., $\beta$-Amyloid1-42, HIV-1Ba-L (Clade B) infection and drugs of abuse induced degeneration in human neuronal cells and protective effects of Ashwagandha (Withania somnifera) and its constituent Withanolide A. PLoS One, 2014. 9(11): p. e112818.

121. Snider, W.D., et al., Neurological complications of acquired immune deficiency syndrome: analysis of 50 patients. Annals of Neurology: Official Journal of the American Neurological Association and the Child Neurology Society, 1983. 14(4): p. $403-418$. 
122. Ghosh, A.K., S. Gemma, and J. Tang, $\beta$-Secretase as a therapeutic target for Alzheimer's disease. Neurotherapeutics, 2008. 5(3): p. 399-408.

123. Guardia-Laguarta, C., M. Pera, and A. Lleo, gamma-Secretase as a therapeutic target in Alzheimer's disease. Current drug targets, 2010. 11(4): p. 506-517.

124. Kretner, B., et al., Generation and deposition of $A \beta 43$ by the virtually inactive presenilin-1 L435F mutant contradicts the presenilin loss-of-function hypothesis of Alzheimer's disease. EMBO molecular medicine, 2016: p. e201505952.

125. Zheng, W.-H., et al., Amyloid $\beta$ peptide induces tau phosphorylation and loss of cholinergic neurons in rat primary septal cultures. Neuroscience, 2002. 115(1): p. 201-211.

126. Echeverria, V., et al., Altered mitogen-activated protein kinase signaling, tau hyperphosphorylation and mild spatial learning dysfunction in transgenic rats expressing the $\beta$-amyloid peptide intracellularly in hippocampal and cortical neurons. Neuroscience, 2004. 129(3): p. 583-592.

127. Ahyayauch, $\mathrm{H}$., et al., Binding of $\beta$-amyloid (1-42) peptide to negatively charged phospholipid membranes in the liquid-ordered state: Modeling and experimental studies. Biophysical journal, 2012. 103(3): p. 453-463.

128. Venkitaramani, D.V., et al., $\beta$-amyloid modulation of synaptic transmission and plasticity. Journal of Neuroscience, 2007. 27(44): p. 11832-11837.

129. Palop, J.J. and L. Mucke, Amyloid- $\beta$-induced neuronal dysfunction in Alzheimer's disease: from synapses toward neural networks. Nature neuroscience, 2010. 13(7): p. 812.

130. $\mathrm{Li}, \mathrm{Y}$., et al., Role of amyloid $\beta$ protein receptors in mediating synaptic plasticity. Biomedical reports, 2017. 6(4): p. 379-386.

131. Butterfield, D.A., A.M. Swomley, and R. Sultana, Amyloid $\beta$-peptide (1-42)induced oxidative stress in Alzheimer disease: importance in disease pathogenesis and progression. Antioxidants \& redox signaling, 2013. 19(8): p. 823835.

132. Arimon, M., et al., Oxidative stress and lipid peroxidation are upstream of amyloid pathology. Neurobiology of disease, 2015. 84: p. 109-119.

133. Cheignon, C., et al., Oxidative stress and the amyloid beta peptide in Alzheimer's disease. Redox Biology, 2018. 14: p. 450-464.

134. Barage, S.H. and K.D. Sonawane, Amyloid cascade hypothesis: pathogenesis and therapeutic strategies in Alzheimer's disease. Neuropeptides, 2015. 52: p. 1-18.

135. Marottoli, F.M., et al., Peripheral inflammation, apolipoprotein E4, and amyloid- $\beta$ Interact to induce cognitive and cerebrovascular dysfunction. ASN neuro, 2017. 9(4): p. 1759091417719201. 
136. Zolezzi, J.M., et al., Alzheimer's disease: relevant molecular and physiopathological events affecting amyloid- $\beta$ brain balance and the putative role of PPARs. Frontiers in aging neuroscience, 2014. 6: p. 176.

137. András, I.E. and M. Toborek, Amyloid beta accumulation in HIV-1-infected brain: The role of the blood brain barrier. IUBMB life, 2013. 65(1): p. 43-49.

138. Martínez-Bonet, M., M.Á. Muñoz-Fernández, and S. Álvarez, HIV-1 increases extracellular amyloid-beta levels through neprilysin regulation in primary cultures of human astrocytes. Journal of cellular physiology, 2018.

139. Achim, C.L., et al., Increased accumulation of intraneuronal amyloid $\beta$ in HIVinfected patients. Journal of Neuroimmune Pharmacology, 2009. 4(2): p. 190-199.

140. Soontornniyomkij, V., et al., Cerebral $\beta$-amyloid deposition predicts HIVassociated neurocognitive disorders in APOE $\varepsilon 4$ carriers. AIDS (London, England), 2012. 26(18): p. 2327.

141. Bagashev, A. and B.E. Sawaya, Roles and functions of HIV-1 Tat protein in the CNS: an overview. Virology journal, 2013. 10(1): p. 358.

142. Daily, A., A. Nath, and L.B. Hersh, Tat peptides inhibit neprilysin. Journal of neurovirology, 2006. 12(3): p. 153-160.

143. Chandra, T., et al., Molecular interactions of the type 1 human immunodeficiency virus transregulatory protein Tat with $\mathrm{N}$-methyl-d-aspartate receptor subunits. Neuroscience, 2005. 134(1): p. 145-153.

144. Tahirov, T.H., et al., Crystal structure of HIV-1 Tat complexed with human P-TEFb. Nature, 2010. 465(7299): p. 747.

145. Hategan, A., et al., HIV Tat protein and amyloid- $\beta$ peptide form multifibrillar structures that cause neurotoxicity. Nature structural \& molecular biology, 2017. 24(4): p. 379.

146. Kanemitsu, H., T. Tomiyama, and H. Mori, Human neprilysin is capable of degrading amyloid $\beta$ peptide not only in the monomeric form but also the pathological oligomeric form. Neuroscience letters, 2003. 350(2): p. 113-116.

147. Ramage, S., et al., Hyperphosphorylated tau and amyloid precursor protein deposition is increased in the brains of young drug abusers. Neuropathology and applied neurobiology, 2005. 31(4): p. 439-448.

148. Dublin, S., et al., Use of analgesics (opioids and nonsteroidal anti-inflammatory drugs) and dementia-related neuropathology in a community-based autopsy cohort. Journal of Alzheimer's Disease, 2017. 58(2): p. 435-448.

149. Jayant, R.D., et al., Novel nanoformulation to mitigate co-effects of drugs of abuse and HIV-1 infection: towards the treatment of NeuroAIDS. Journal of neurovirology, 2017. 23(4): p. 603-614. 
150. Javadi-Paydar, M., et al., HIV-1 and cocaine disrupt dopamine reuptake and medium spiny neurons in female rat striatum. PloS one, 2017. 12(11): p. e0188404.

151. Meade, C.S., et al., Cocaine dependence modulates the effect of HIV infection on brain activation during intertemporal decision making. Drug and alcohol dependence, 2017. 178: p. 443-451.

152. Wakim, K.-M., et al., White Matter changes in hiV+ Women with a history of cocaine Dependence. Frontiers in neurology, 2017. 8: p. 562.

153. Mirjalili, M.H., et al., Steroidal lactones from Withania somnifera, an ancient plant for novel medicine. Molecules, 2009. 14(7): p. 2373-2393.

154. Bargagna-Mohan, P., et al., The tumor inhibitor and antiangiogenic agent withaferin A targets the intermediate filament protein vimentin. Chemistry \& biology, 2007. 14(6): p. 623-634.

155. Mohan, R., et al., Withaferin A is a potent inhibitor of angiogenesis. Angiogenesis, 2004. 7(2): p. 115-122.

156. Challa, A.A., et al., Withaferin-A reduces type I collagen expression in vitro and inhibits development of myocardial fibrosis in vivo. PloS one, 2012. 7(8): p. e42989.

157. Mohan, R. and P. Bargagna-Mohan, The use of Withaferin A to study intermediate filaments, in Methods in enzymology. 2016, Elsevier. p. 187-218.

158. Yu, Y., et al., Withaferin A targets heat shock protein 90 in pancreatic cancer cells. Biochemical pharmacology, 2010. 79(4): p. 542-551.

159. Choi, B.Y. and B.-W. Kim, Withaferin-A inhibits colon cancer cell growth by blocking STAT3 transcriptional activity. Journal of cancer prevention, 2015. 20(3): p. 185.

160. Lee, I.-C. and B.Y. Choi, Withaferin-A-a natural anticancer agent with pleitropic mechanisms of action. International journal of molecular sciences, 2016. 17(3): p. 290.

161. Rah, B., et al., A novel MMP-2 inhibitor 3-azidowithaferin A (3-azidoWA) abrogates cancer cell invasion and angiogenesis by modulating extracellular Par-4. PloS one, 2012. 7(9): p. e44039.

162. Park, H.J., et al., Withaferin A induces apoptosis and inhibits adipogenesis in 3T3L1 adipocytes. Biofactors, 2008. 33(2): p. 137-148.

163. Heaton, R., et al., HIV-associated neurocognitive disorders persist in the era of potent antiretroviral therapy CHARTER Study. Neurology, 2010. 75(23): p. 20872096. 
164. Heaton, R.K., et al., HIV-associated neurocognitive disorders before and during the era of combination antiretroviral therapy: differences in rates, nature, and predictors. Journal of neurovirology, 2011. 17(1): p. 3-16.

165. Ellis, R.J., et al., Continued high prevalence and adverse clinical impact of human immunodeficiency virus-associated sensory neuropathy in the era of combination antiretroviral therapy: the CHARTER Study. Archives of neurology, 2010. 67(5): p. $552-558$.

166. Van Marum, R.J., Current and future therapy in Alzheimer's disease. Fundamental \& clinical pharmacology, 2008. 22(3): p. 265-274.

167. Sharma, R.A., W.P. Steward, and A.J. Gescher, Pharmacokinetics and pharmacodynamics of curcumin, in The molecular targets and therapeutic uses of curcumin in health and disease. 2007, Springer. p. 453-470.

168. Ringman, J.M., et al., A potential role of the curry spice curcumin in Alzheimer's disease. Current Alzheimer Research, 2005. 2(2): p. 131-136.

169. Ishrat, T., et al., Amelioration of cognitive deficits and neurodegeneration by curcumin in rat model of sporadic dementia of Alzheimer's type (SDAT). European Neuropsychopharmacology, 2009. 19(9): p. 636-647.

170. Anand, P., et al., Bioavailability of curcumin: problems and promises. Molecular pharmaceutics, 2007. 4(6): p. 807-818.

171. Tohda, C., T. Kuboyama, and K. Komatsu, Dendrite extension by methanol extract of Ashwagandha (roots of Withania somnifera) in SK-N-SH cells. Neuroreport, 2000. 11(9): p. 1981-1985.

172. Zhao, J., et al., Withanolide derivatives from the roots of Withania somnifera and their neurite outgrowth activities. Chemical and pharmaceutical bulletin, 2002. 50(6): p. 760-765.

173. Nuovo, G.J., et al., In situ detection of PCR-amplified HIV-1 nucleic acids in lymph nodes and peripheral blood in patients with asymptomatic HIV-1 infection and advanced-stage AIDS. Journal of acquired immune deficiency syndromes, 1994. 7(9): p. 916-923.

174. Nath, A., et al., Identification of a human immunodeficiency virus type 1 Tat epitope that is neuroexcitatory and neurotoxic. Journal of virology, 1996. 70(3): p. 14751480.

175. Merino, J.J., et al., HIV-1 neuropathogenesis: therapeutic strategies against neuronal loss induced by gp120/Tat glycoprotein in the central nervous system. Revista de neurologia, 2011. 52(2): p. 101-111.

176. Rempel, H.C. and L. Pulliam, HIV-1 Tat inhibits neprilysin and elevates amyloid $\beta$. Aids, 2005. 19(2): p. 127-135. 
177. Giunta, B., et al., HIV-1 Tat contributes to Alzheimer's disease-like pathology in PSAPP mice. International journal of clinical and experimental pathology, 2009. 2(5): p. 433.

178. Aksenov, M., et al., HIV-1 protein-mediated amyloidogenesis in rat hippocampal cell cultures. Neuroscience letters, 2010. 475(3): p. 174-178.

179. Chen, X., et al., Endolysosome involvement in HIV-1 transactivator proteininduced neuronal amyloid beta production. Neurobiology of aging, 2013. 34(10): p. $2370-2378$.

180. Fiala, M., et al., Cocaine enhances monocyte migration across the blood-brain barrier, in Drugs of Abuse, Immunomodulation, and Aids. 1998, Springer. p. 199205.

181. Gannon, P., M.Z. Khan, and D.L. Kolson, Current understanding of HIV-associated neurocognitive disorders pathogenesis. Current opinion in neurology, 2011. 24(3): p. 275.

182. Buch, S., et al., Cocaine and HIV-1 interplay in CNS: cellular and molecular mechanisms. Current HIV research, 2012. 10(5): p. 425-428.

183. Liu, S.-J., et al., Alzheimer-like phosphorylation of tau and neurofilament induced by cocaine in vivo. Acta pharmacologica Sinica, 2003. 24(6): p. 512-518.

184. ladecola, C., The pathobiology of vascular dementia. Neuron, 2013. 80(4): p. 844866.

185. Justin, B.N., M. Turek, and A.M. Hakim, Heart disease as a risk factor for dementia. Clinical epidemiology, 2013. 5: p. 135.

186. Kivipelto, M., et al., Obesity and vascular risk factors at midlife and the risk of dementia and Alzheimer disease. Archives of neurology, 2005. 62(10): p. 15561560.

187. Beeri, M.S., et al., Diabetes mellitus in midlife and the risk of dementia three decades later. Neurology, 2004. 63(10): p. 1902-1907.

188. Tilvis, R.S., et al., Predictors of cognitive decline and mortality of aged people over a 10-year period. The Journals of Gerontology Series A: Biological Sciences and Medical Sciences, 2004. 59(3): p. M268-M274.

189. Qiu, C., et al., Heart failure and risk of dementia and Alzheimer disease: a population-based cohort study. Archives of Internal Medicine, 2006. 166(9): p. 1003-1008.

190. Santangeli, P., et al., Atrial fibrillation and the risk of incident dementia: a metaanalysis. Heart Rhythm, 2012. 9(11): p. 1761-1768. e2. 
191. Dotson, V.M., M.A. Beydoun, and A.B. Zonderman, Recurrent depressive symptoms and the incidence of dementia and mild cognitive impairment. Neurology, 2010. 75(1): p. 27-34.

192. Fleminger, S., Head injury as a risk factor for Alzheimer's disease.(BNPA Abstracts: Recovering From Head Injury). Journal of Neurology, Neurosurgery and Psychiatry, 2003. 74(6): p. 832-833.

193. Guo, Z., et al., Head injury and the risk of AD in the MIRAGE study. Neurology, 2000. 54(6): p. 1316-1323.

194. van Kooten, F. and P.J. Koudstaal, Epidemiology of post-stroke dementia. Pathophysiology of Haemostasis and Thrombosis, 1998. 28(3-4): p. 124-133.

195. Pendlebury, S.T. and P.M. Rothwell, Prevalence, incidence, and factors associated with pre-stroke and post-stroke dementia: a systematic review and meta-analysis. The Lancet Neurology, 2009. 8(11): p. 1006-1018.

196. Anstey, K.J., et al., Smoking as a risk factor for dementia and cognitive decline: a meta-analysis of prospective studies. American journal of epidemiology, 2007. 166(4): p. 367-378.

197. Anttila, T., et al., Alcohol drinking in middle age and subsequent risk of mild cognitive impairment and dementia in old age: a prospective population based study. Bmj, 2004. 329(7465): p. 539.

198. Mukamal, K.J., et al., Prospective study of alcohol consumption and risk of dementia in older adults. Jama, 2003. 289(11): p. 1405-1413.

199. Swiger, K.J., et al. Statins and cognition: a systematic review and meta-analysis of short-and long-term cognitive effects. in Mayo Clinic Proceedings. 2013. Elsevier.

200. Wong, W.B., et al., Statins in the prevention of dementia and Alzheimer's disease: a meta-analysis of observational studies and an assessment of confounding. Pharmacoepidemiology and drug safety, 2013. 22(4): p. 345-358.

201. Schmidt, R., et al., Early inflammation and dementia: a 25-year follow-up of the Honolulu-Asia Aging Study. Annals of neurology, 2002. 52(2): p. 168-174.

202. Schott, J.M. and T. Revesz, Inflammation in Alzheimer's disease: insights from immunotherapy. Brain, 2013. 136(9): p. 2654-2656.

203. Akiyama, H., et al., Inflammation and Alzheimer's disease. Neurobiology of aging, 2000. 21(3): p. 383-421.

204. Tuppo, E.E. and H.R. Arias, The role of inflammation in Alzheimer's disease. The international journal of biochemistry \& cell biology, 2005. 37(2): p. 289-305. 
205. Iversen, L.L., et al., The toxicity in vitro of beta-amyloid protein. Biochemical Journal, 1995. 311(Pt 1): p. 1.

206. Hohsfield, L.A. and C. Humpel, Migration of blood cells to $\beta$-amyloid plaques in Alzheimer's disease. Experimental gerontology, 2015. 65: p. 8-15.

207. Zhang, K., et al., CXCL1 contributes to $\beta$-amyloid-induced transendothelial migration of monocytes in Alzheimer's disease. PLoS One, 2013. 8(8): p. e72744.

208. Akiyama, H., et al., Webster. S.; Wegrzyniak, B.; Wenk, G.; Wyss-Coray, T. Inflammation and Alzheimer's disease. Neurobiol. Aging, 2000. 21(3): p. 383-421.

209. Kitazawa, M., T.R. Yamasaki, and F.M. Laferla, Microglia as a Potential Bridge between the Amyloid $\beta$-Peptide and Tau. Annals of the New York Academy of Sciences, 2004. 1035(1): p. 85-103.

210. Bauernfeind, F., et al., Inflammasomes: current understanding and open questions. Cellular and Molecular Life Sciences, 2011. 68(5): p. 765-783.

211. Agostini, L., et al., NALP3 forms an IL-1 $\beta$-processing inflammasome with increased activity in Muckle-Wells autoinflammatory disorder. Immunity, 2004. 20(3): p. 319-325.

212. Masters, S.L., et al., Activation of the NLRP3 inflammasome by islet amyloid polypeptide provides a mechanism for enhanced IL-1 $\beta$ in type 2 diabetes. Nature immunology, 2010. 11(10): p. 897.

213. Latz, E., The inflammasomes: mechanisms of activation and function. Current opinion in immunology, 2010. 22(1): p. 28-33.

214. Goldgaber, D., et al., Interleukin 1 regulates synthesis of amyloid beta-protein precursor mRNA in human endothelial cells. Proceedings of the National Academy of Sciences, 1989. 86(19): p. 7606-7610.

215. Griffin, W.S.T., et al., Interleukin-1 expression in different plaque types in Alzheimer's disease: significance in plaque evolution. Journal of Neuropathology \& Experimental Neurology, 1995. 54(2): p. 276-281.

216. Kitazawa, M., et al., Blocking IL-1 signaling rescues cognition, attenuates tau pathology, and restores neuronal $\beta$-catenin pathway function in an Alzheimer's disease model. The Journal of Immunology, 2011: p. 1100620.

217. Sheng, J., et al., Interleukin-1 promotes expression and phosphorylation of neurofilament and tau proteins in vivo. Experimental neurology, 2000. 163(2): p. 388-391.

218. Li, Y., et al., Interleukin-1 mediates pathological effects of microglia on tau phosphorylation and on synaptophysin synthesis in cortical neurons through a p38MAPK pathway. Journal of Neuroscience, 2003. 23(5): p. 1605-1611. 
219. Coll, R.C. and L.A. O'Neill, The cytokine release inhibitory drug CRID3 targets ASC oligomerisation in the NLRP3 and AIM2 inflammasomes. PloS one, 2011. 6(12): p. e29539.

220. MERAZ RIOS, M.A., et al., Inflammatory process in Alzheimer's Disease. Frontiers in integrative neuroscience, 2013. 7: p. 59.

221. Lawrence, T., The nuclear factor NF-kB pathway in inflammation. Cold Spring Harbor perspectives in biology, 2009: p. a001651.

222. Karran, E. and B. De Strooper, The amyloid cascade hypothesis: are we poised for success or failure? Journal of neurochemistry, 2016. 139: p. 237-252.

223. Qiang, W., et al., Structural variation in amyloid- $\beta$ fibrils from Alzheimer's disease clinical subtypes. Nature, 2017. 541(7636): p. 217.

224. Chen, Y., et al., Hybrids of oxoisoaporphine-tetrahydroisoquinoline: novel multitarget inhibitors of inflammation and amyloid- $\beta$ aggregation in Alzheimer's disease. Molecular diversity, 2019: p. 1-14.

225. Cai, Z., M.D. Hussain, and L.-J. Yan, Microglia, neuroinflammation, and betaamyloid protein in Alzheimer's disease. International Journal of Neuroscience, 2014. 124(5): p. 307-321.

226. Doens, D. and P.L. Fernández, Microglia receptors and their implications in the response to amyloid $\beta$ for Alzheimer's disease pathogenesis. Journal of neuroinflammation, 2014. 11(1): p. 48.

227. Zhang, J.-X., et al., Luteolin Inhibits Fibrillary $\beta$-Amyloid1-40-Induced Inflammation in a Human Blood-Brain Barrier Model by Suppressing the p38 MAPK-Mediated NF-KB Signaling Pathways. Molecules, 2017. 22(3): p. 334.

228. Hayden, M.S. and S. Ghosh, Shared principles in NF-kB signaling. Cell, 2008. 132(3): p. 344-362.

229. Perkins, N.D., Post-translational modifications regulating the activity and function of the nuclear factor kappa B pathway. Oncogene, 2006. 25(51): p. 6717.

230. Sun, S.C., The noncanonical NF-kB pathway. Immunological reviews, 2012. 246(1): p. $125-140$.

231. Tak, P.P. and G.S. Firestein, NF-kB: a key role in inflammatory diseases. The Journal of clinical investigation, 2001. 107(1): p. 7-11.

232. Karin, M., Y. Yamamoto, and Q.M. Wang, The IKK NF-KB system: a treasure trove for drug development. Nature reviews Drug discovery, 2004. 3(1): p. 17.

233. Kuehn, H.S., et al., Novel nonsense gain-of-function NFKB2 mutations associated with a combined immunodeficiency phenotype. Blood, 2017: p. blood-2017-05782177 . 
234. De Wit, H., et al., Regulation of p100 (NFKB2) expression in human monocytes in response to inflammatory mediators and lymphokines. Leukemia, 1998. 12(3): p. 363.

235. Mulero, M.C., et al., Protein Cofactors Are Essential for High-Affinity DNA Binding by the Nuclear Factor kB RelA Subunit. Biochemistry, 2018. 57(20): p. 2943-2957.

236. Scherer, D.C., et al., Signal-induced degradation of I kappa B alpha requires sitespecific ubiquitination. Proceedings of the National Academy of Sciences, 1995. 92(24): p. 11259-11263.

237. Li, Z. and G.J. Nabel, A new member of the I kappaB protein family, I kappaB epsilon, inhibits RelA (p65)-mediated NF-kappaB transcription. Molecular and cellular biology, 1997. 17(10): p. 6184-6190.

238. Andrei, C., et al., Phospholipases C and A2 control lysosome-mediated IL-1 $\beta$ secretion: implications for inflammatory processes. Proceedings of the National Academy of Sciences, 2004. 101(26): p. 9745-9750.

239. Dubey, S., et al., Withaferin a associated Differential regulation of inflammatory cytokines. Frontiers in immunology, 2018. 9: p. 195.

240. McCormick, T.S., S.R. Stevens, and K. Kang, Macrophages and cutaneous inflammation. Nature biotechnology, 2000. 18(1): p. 25.

241. Ren, K. and R. Torres, Role of interleukin-1 $\beta$ during pain and inflammation. Brain research reviews, 2009. 60(1): p. 57-64.

242. Boraschi, D. and A. Tagliabue. The interleukin-1 receptor family. in Seminars in immunology. 2013. Elsevier.

243. Cullen, S.P., et al., Diverse activators of the NLRP3 inflammasome promote IL-1 $\beta$ secretion by triggering necrosis. Cell reports, 2015. 11(10): p. 1535-1548.

244. Karan, D. and S. Dubey, From inflammation to prostate cancer: the role of inflammasomes. Advances in Urology, 2016. 2016.

245. Piccioli, P. and A. Rubartelli. The secretion of IL-1 $\beta$ and options for release. in Seminars in immunology. 2013. Elsevier.

246. Dinarello, C.A., Interleukin-1 in the pathogenesis and treatment of inflammatory diseases. Blood, 2011. 117(14): p. 3720-3732.

247. Dyall, S., Amyloid-beta peptide, oxidative stress and inflammation in alzheimer's disease: Potential neuroprotective effects of omega-3 polyunsaturated fatty acids. International Journal of Alzheimer's Disease, 2010. 2010.

248. Koning, G.A. and G. Storm, Targeted drug delivery systems for the intracellular delivery of macromolecular drugs. Drug discovery today, 2003. 11(8): p. 482-483. 
249. Metselaar, J.M. and G. Storm, Liposomes in the treatment of inflammatory disorders. Expert opinion on drug delivery, 2005. 2(3): p. 465-476.

250. Ding, B.-S., et al., Advanced drug delivery systems that target the vascular endothelium. Molecular interventions, 2006. 6(2): p. 98.

251. Sercombe, L., et al., Advances and challenges of liposome assisted drug delivery. Frontiers in pharmacology, 2015. 6: p. 286.

252. Srinivasan, B., et al., TEER measurement techniques for in vitro barrier model systems. Journal of laboratory automation, 2015. 20(2): p. 107-126.

253. Tarawneh, R. and D.M. Holtzman, The clinical problem of symptomatic Alzheimer disease and mild cognitive impairment. Cold Spring Harbor perspectives in medicine, 2012: p. a006148.

254. Feldman, H.H. and C. Jacova, Mild cognitive impairment. The American Journal of Geriatric Psychiatry, 2005. 13(8): p. 645-655.

255. Prince, M., et al., The global prevalence of dementia: a systematic review and metaanalysis. Alzheimer's \& dementia, 2013. 9(1): p. 63-75. e2.

256. Hugo, J. and M. Ganguli, Dementia and cognitive impairment: epidemiology, diagnosis, and treatment. Clinics in geriatric medicine, 2014. 30(3): p. 421-442.

257. Brookmeyer, R., et al., Survival following a diagnosis of Alzheimer disease. Archives of neurology, 2002. 59(11): p. 1764-1767.

258. Association, A.P., Diagnostic and statistical manual of mental disorders (DSM-5®). 2013: American Psychiatric Pub.

259. Pardridge, W.M., The blood-brain barrier: bottleneck in brain drug development. NeuroRx, 2005. 2(1): p. 3-14.

260. Abbott, N.J., Blood-brain barrier structure and function and the challenges for CNS drug delivery. Journal of inherited metabolic disease, 2013. 36(3): p. 437-449.

261. Henderson, J. and M. Piquette-Miller, Blood-brain barrier: An impediment to neuropharmaceuticals. Clinical Pharmacology \& Therapeutics, 2015. 97(4): p. 308-313.

262. De Lange, E. and M. Hammarlund-Udenaes, Translational aspects of blood-brain barrier transport and central nervous system effects of drugs: from discovery to patients. Clinical Pharmacology \& Therapeutics, 2015. 97(4): p. 380-394.

263. S Hersh, D., et al., Evolving drug delivery strategies to overcome the blood brain barrier. Current pharmaceutical design, 2016. 22(9): p. 1177-1193.

264. Tomitaka, A., et al., Hybrid magneto-plasmonic liposomes for multimodal imageguided and brain-targeted HIV treatment. Nanoscale, 2018. 10(1): p. 184-194. 
265. Tomitaka, A., et al., Development of magneto-plasmonic nanoparticles for multimodal image-guided therapy to the brain. Nanoscale, 2017. 9(2): p. 764-773.

266. Mudshinge, S.R., et al., Nanoparticles: emerging carriers for drug delivery. Saudi pharmaceutical journal, 2011. 19(3): p. 129-141.

267. de Smet, M., et al., SPECT/CT imaging of temperature-sensitive liposomes for MR-image guided drug delivery with high intensity focused ultrasound. Journal of controlled release, 2013. 169(1-2): p. 82-90.

268. Mieszawska, A.J., et al., Synthesis of polymer-lipid nanoparticles for imageguided delivery of dual modality therapy. Bioconjugate chemistry, 2013. 24(9): p. 1429-1434.

269. He, Q., et al., Towards improvements for penetrating the blood-brain barrierrecent progress from a material and pharmaceutical perspective. Cells, 2018. 7(4): p. 24.

270. Fu, B.M., Transport Across the Blood-Brain Barrier, in Molecular, Cellular, and Tissue Engineering of the Vascular System. 2018, Springer. p. 235-259.

271. De, A.B. and D. Breimer, The blood-brain barrier: clinical implications for drug delivery to the brain. Journal of the Royal College of Physicians of London, 1994. 28(6): p. 502-506.

272. Bicker, J., et al., Blood-brain barrier models and their relevance for a successful development of CNS drug delivery systems: a review. European Journal of Pharmaceutics and Biopharmaceutics, 2014. 87(3): p. 409-432.

273. Wu, S.-Y., et al., Effects of the microbubble shell physicochemical properties on ultrasound-mediated drug delivery to the brain. Journal of Controlled Release, 2015. 212: p. 30-40.

274. Rautio, J., et al., Prodrug approaches for CNS delivery. The AAPS journal, 2008. 10(1): p. 92-102.

275. Hitchcock, S.A. and L.D. Pennington, Structure- brain exposure relationships. Journal of medicinal chemistry, 2006. 49(26): p. 7559-7583.

276. Saxena, S.K., S. Tiwari, and M.P. Nair, Nanotherapeutics: emerging competent technology in neuroAIDS and CNS drug delivery. Nanomedicine, 2012. 7(7): p. 941-944.

277. Vieira, D.B. and L.F. Gamarra, Getting into the brain: liposome-based strategies for effective drug delivery across the blood-brain barrier. International journal of nanomedicine, 2016. 11: p. 5381.

278. Lai, F., A.M. Fadda, and C. Sinico, Liposomes for brain delivery. Expert opinion on drug delivery, 2013. 10(7): p. 1003-1022. 
279. Noble, G.T., et al., Ligand-targeted liposome design: challenges and fundamental considerations. Trends in biotechnology, 2014. 32(1): p. 32-45.

280. Ross, C., et al., Liposome delivery systems for the treatment of Alzheimer's disease. International journal of nanomedicine, 2018. 13: p. 8507.

281. Jiang, X., et al., Enhancement of radiotherapy efficacy by pleiotropic liposomes encapsulated paclitaxel and perfluorotributylamine. Drug delivery, 2017. 24(1): $p$. 1419-1428.

282. Costa, C., et al., Nose-to-brain delivery of lipid-based nanosystems for epileptic seizures and anxiety crisis. Journal of Controlled Release, 2019.

283. Pires, P.C. and A.O. Santos, Nanosystems in nose-to-brain drug delivery: A review of non-clinical brain targeting studies. Journal of Controlled Release, 2018. 270: $p$. $89-100$

284. Stathopoulos, G. and T. Boulikas, Lipoplatin formulation review article. Journal of drug delivery, 2012. 2012.

285. Lee, S., et al., Liposomes to target peripheral neurons and Schwann cells. PloS one, 2013. 8(11): p. e78724.

286. Persidsky, Y., et al., A model for monocyte migration through the blood-brain barrier during HIV-1 encephalitis. The Journal of Immunology, 1997. 158(7): p. 3499-3510.

287. Saiyed, Z.M., N.H. Gandhi, and M.P. Nair, Magnetic nanoformulation of azidothymidine 5'-triphosphate for targeted delivery across the blood-brain barrier. International journal of nanomedicine, 2010. 5: p. 157.

288. Ding, H., et al., Enhanced blood-brain barrier transmigration using a novel transferrin embedded fluorescent magneto-liposome nanoformulation. Nanotechnology, 2014. 25(5): p. 055101.

289. Ong, S.G.M., et al., Evaluation of extrusion technique for nanosizing liposomes. Pharmaceutics, 2016. 8(4): p. 36.

290. Lapinski, M.M., et al., Comparison of liposomes formed by sonication and extrusion: rotational and translational diffusion of an embedded chromophore. Langmuir, 2007. 23(23): p. 11677-11683.

291. Winterhalter, M. and D.D. Lasic, Liposome stability and formation: experimental parameters and theories on the size distribution. Chemistry and physics of lipids, 1993. 64(1-3): p. 35-43.

292. Hardy, J. and D.J. Selkoe, The amyloid hypothesis of Alzheimer's disease: progress and problems on the road to therapeutics. science, 2002. 297(5580): p. 353-356. 
293. Rodrigue, K., et al., $\beta$-Amyloid burden in healthy aging: regional distribution and cognitive consequences. Neurology, 2012. 78(6): p. 387-395.

294. Tomiyama, T., Involvement of beta-amyloid in the etiology of Alzheimer's disease. Brain and nerve= Shinkei kenkyu no shinpo, 2010. 62(7): p. 691-699.

295. Güntert, A., H. Döbeli, and B. Bohrmann, High sensitivity analysis of amyloid-beta peptide composition in amyloid deposits from human and PS2APP mouse brain. Neuroscience, 2006. 143(2): p. 461-475.

296. Scheff, S.W., et al., Synapse stability in the precuneus early in the progression of Alzheimer's disease. Journal of Alzheimer's Disease, 2013. 35(3): p. 599-609.

297. Lesné, S.E., et al., Brain amyloid- $\beta$ oligomers in ageing and Alzheimer's disease. Brain, 2013. 136(5): p. 1383-1398.

298. Scheff, S.W., et al., Synaptic change in the posterior cingulate gyrus in the progression of Alzheimer's disease. Journal of Alzheimer's Disease, 2015. 43(3): p. 1073-1090.

299. Scheff, S.W., et al., Synaptic loss in the inferior temporal gyrus in mild cognitive impairment and Alzheimer's disease. Journal of Alzheimer's Disease, 2011. 24(3): p. 547-557.

300. Roy, U., et al., Pharmacodynamic and antiretroviral activities of combination nanoformulated antiretrovirals in HIV-1-infected human peripheral blood lymphocyte-reconstituted mice. The Journal of infectious diseases, 2012. 206(10): p. 1577-1588.

301. Bhardwaj, V. and A. Kaushik, Biomedical Applications of Nanotechnology and Nanomaterials. 2017, Multidisciplinary Digital Publishing Institute.

302. Venkatraman, S.S., et al., Polymer-and liposome-based nanoparticles in targeted drug delivery. Front Biosci (Schol Ed), 2010. 2: p. 801-14.

303. Kaushik, A., et al., Magnetically guided central nervous system delivery and toxicity evaluation of magneto-electric nanocarriers. Scientific reports, 2016. 6: p. 25309.

304. Kaushik, A., et al., The potential of magneto-electric nanocarriers for drug delivery. Expert opinion on drug delivery, 2014. 11(10): p. 1635-1646.

305. Kaushik, A., et al., Investigation of ac-magnetic field stimulated nanoelectroporation of magneto-electric nano-drug-carrier inside CNS cells. Scientific reports, 2017. 7: p. 45663.

306. Saiyed, Z.M., N.H. Gandhi, and M.P. Nair, AZT 5'-triphosphate nanoformulation suppresses human immunodeficiency virus type 1 replication in peripheral blood mononuclear cells. Journal of neurovirology, 2009. 15(4): p. 343-347. 
307. Demirdirek, B., et al., Polymerized Drugs-A Novel Approach to Controlled Release Systems. Polymers for Biomedicine: Synthesis, Characterization, and Applications, 2017: p. 357.

308. Rejman, J., et al., Size-dependent internalization of particles via the pathways of clathrin-and caveolae-mediated endocytosis. Biochemical Journal, 2004. 377(1): p. $159-169$.

309. De Camilli, P. and K. Takei, Molecular mechanisms in synaptic vesicle endocytosis and recycling. Neuron, 1996. 16(3): p. 481-486.

310. Donaldson, J.G., N. Porat-Shliom, and L.A. Cohen, Clathrin-independent endocytosis: a unique platform for cell signaling and PM remodeling. Cellular signalling, 2009. 21(1): p. 1-6.

311. Allen, T.M. and P.R. Cullis, Liposomal drug delivery systems: from concept to clinical applications. Advanced drug delivery reviews, 2013. 65(1): p. 36-48.

312. Raju, G.S.R., et al., Multifunctional nanoparticles: Recent progress in cancer therapeutics. Chemical Communications, 2015. 51(68): p. 13248-13259.

313. Bozzuto, G. and A. Molinari, Liposomes as nanomedical devices. International journal of nanomedicine, 2015. 10: p. 975.

314. Schnyder, A. and J. Huwyler, Drug transport to brain with targeted liposomes. NeuroRx, 2005. 2(1): p. 99-107.

315. Chaudhuri, T.K. and S. Paul, Protein-misfolding diseases and chaperone-based therapeutic approaches. The FEBS journal, 2006. 273(7): p. 1331-1349.

316. M Ashraf, G., et al., Protein misfolding and aggregation in Alzheimer's disease and type 2 diabetes mellitus. CNS \& Neurological Disorders-Drug Targets (Formerly Current Drug Targets-CNS \& Neurological Disorders), 2014. 13(7): p. 1280-1293.

317. Sinha, S. and I. Lieberburg, Cellular mechanisms of $\beta$-amyloid production and secretion. Proceedings of the National Academy of Sciences, 1999. 96(20): p. 11049-11053.

318. Haass, C., et al., Amyloid $\beta$-peptide is produced by cultured cells during normal metabolism. Nature, 1992. 359(6393): p. 322.

319. Choi, S.H., et al., A three-dimensional human neural cell culture model of Alzheimer's disease. Nature, 2014. 515(7526): p. 274.

320. Adolfsson, O., et al., An effector-reduced anti- $\beta$-amyloid $(A \beta)$ antibody with unique $a \beta$ binding properties promotes neuroprotection and glial engulfment of $A \beta$. Journal of Neuroscience, 2012. 32(28): p. 9677-9689.

321. Salloway, S., et al., Two phase 3 trials of bapineuzumab in mild-to-moderate Alzheimer's disease. New England Journal of Medicine, 2014. 370(4): p. 322-333. 
322. Villemagne, V.L., et al., Amyloid $\beta$ deposition, neurodegeneration, and cognitive decline in sporadic Alzheimer's disease: a prospective cohort study. The Lancet Neurology, 2013. 12(4): p. 357-367.

323. Mullard, A., Sting of Alzheimer's failures offset by upcoming prevention trials. 2012, Nature Publishing Group.

324. Shimada, H., DIAN BRAIN and NERVE, 2013. 65(10): p. 1179-1184.

325. Reiman, E.M., et al., Alzheimer's Prevention Initiative: a plan to accelerate the evaluation of presymptomatic treatments. Journal of Alzheimer's Disease, 2011. 26(s3): p. 321-329.

326. Crespi, G.A., et al., Molecular basis for mid-region amyloid- $\beta$ capture by leading Alzheimer's disease immunotherapies. Scientific reports, 2015. 5: p. 9649.

327. Miles, L.A., et al., Amyloid- $\beta$-anti-amyloid- $\beta$ complex structure reveals an extended conformation in the immunodominant B-cell epitope. Journal of molecular biology, 2008. 377(1): p. 181-192.

328. Gardberg, A.S., et al., Molecular basis for passive immunotherapy of Alzheimer's disease. Proceedings of the National Academy of Sciences, 2007. 104(40): p. $15659-15664$

329. Bohrmann, B., et al., Gantenerumab: a novel human anti-A $\beta$ antibody demonstrates sustained cerebral amyloid- $\beta$ binding and elicits cell-mediated removal of human amyloid- $\beta$. Journal of Alzheimer's Disease, 2012. 28(1): p. 4969.

330. Basi, G.S., et al., Structural correlates of antibodies associated with acute reversal of amyloid $\beta$-related behavioral deficits in a mouse model of Alzheimer disease. Journal of Biological Chemistry, 2010. 285(5): p. 3417-3427.

331. Miles, L.A., et al., Bapineuzumab captures the N-terminus of the Alzheimer's disease amyloid-beta peptide in a helical conformation. Scientific reports, 2013. 3: p. 1302.

332. La Porte, S.L., et al., Structural basis of C-terminal $\beta$-amyloid peptide binding by the antibody ponezumab for the treatment of Alzheimer's disease. Journal of molecular biology, 2012. 421(4-5): p. 525-536.

333. Colvin, M.T., et al., Atomic resolution structure of monomorphic Aß42 amyloid fibrils. Journal of the American Chemical Society, 2016. 138(30): p. 9663-9674.

334. Phillips, J.C., et al., Scalable molecular dynamics with NAMD. J Comput Chem, 2005. 26(16): p. 1781-802.

335. Huang, J. and A.D. MacKerell, Jr., CHARMM36 all-atom additive protein force field: validation based on comparison to NMR data. J Comput Chem, 2013. 34(25): p. 2135-45. 
336. Schmidt, M., et al., Peptide dimer structure in an Abeta(1-42) fibril visualized with cryo-EM. Proc Natl Acad Sci U S A, 2015. 112(38): p. 11858-63.

337. Zhang, R., et al., Interprotofilament interactions between Alzheimer's Abeta1-42 peptides in amyloid fibrils revealed by cryoEM. Proc Natl Acad Sci U S A, 2009. 106(12): p. 4653-8.

338. Gremer, L., et al., Fibril structure of amyloid-beta(1-42) by cryo-electron microscopy. Science, 2017. 358(6359): p. 116-119.

339. Steckmann, T., et al., Cooperative structural transitions in amyloid-like aggregation. J Chem Phys, 2017. 146(13): p. 135103.

340. Jucker, M. and L.C. Walker, Self-propagation of pathogenic protein aggregates in neurodegenerative diseases. Nature, 2013. 501(7465): p. 45-51.

341. Trott, O. and A.J. Olson, AutoDock Vina: improving the speed and accuracy of docking with a new scoring function, efficient optimization, and multithreading. $J$ Comput Chem, 2010. 31(2): p. 455-61.

342. Sandhaus, S., P.P. Chapagain, and Y.C. Tse-Dinh, Discovery of novel bacterial topoisomerase I inhibitors by use of in silico docking and in vitro assays. Sci Rep, 2018. 8(1): p. 1437.

343. Legleiter, J., et al., Effect of different anti-A $\beta$ antibodies on $A \beta$ fibrillogenesis as assessed by atomic force microscopy. Journal of molecular biology, 2004. 335(4): p. 997-1006.

344. Jadhav, P.B., A.R. Yadav, and M.G. Gore, Concept of drug likeness in pharmaceutical research. Int. J. Pharm. Biol. Sci, 2015. 6: p. 142-154.

345. Rajasekhar, K., M. Chakrabarti, and T. Govindaraju, Function and toxicity of amyloid beta and recent therapeutic interventions targeting amyloid beta in Alzheimer's disease. Chemical Communications, 2015. 51(70): p. 13434-13450.

346. Wilkins, H.M. and R.H. Swerdlow, Amyloid precursor protein processing and bioenergetics. Brain research bulletin, 2017. 133: p. 71-79.

347. Arbor, S.C., M. LaFontaine, and M. Cumbay, Focus: The Aging Brain: Amyloidbeta Alzheimer targets-protein processing, lipid rafts, and amyloid-beta pores. The Yale journal of biology and medicine, 2016. 89(1): p. 5.

348. Hamley, I.W., The amyloid beta peptide: a chemist's perspective. Role in Alzheimer's and fibrillization. Chemical reviews, 2012. 112(10): p. 5147-5192.

349. Ling, Y., K. Morgan, and N. Kalsheker, Amyloid precursor protein (APP) and the biology of proteolytic processing: relevance to Alzheimer's disease. The international journal of biochemistry \& cell biology, 2003. 35(11): p. 1505-1535. 
350. Zhang, Y.-w., et al., APP processing in Alzheimer's disease. Molecular brain, 2011. $4(1):$ p. 3.

351. Jiang, S., et al., Trafficking regulation of proteins in Alzheimer's disease. Molecular neurodegeneration, 2014. 9(1): p. 6.

352. Amihăesei, I., E. Cojocarut, and O. Mungiu, Alzheimer--certitudes and hypotheses. Revista medico-chirurgicala a Societatii de Medici si Naturalisti din Iasi, 2013. 117(1): p. 119-126.

353. Luna, S., D.J. Cameron, and D.W. Ethell, Amyloid- $\beta$ and APP deficiencies cause severe cerebrovascular defects: Important work for an old villain. PLoS One, 2013. 8(9): p. e75052.

354. Morris, J.C., et al., APOE predicts amyloid-beta but not tau Alzheimer pathology in cognitively normal aging. Annals of neurology, 2010. 67(1): p. 122-131.

355. Villemagne, V.L., et al., $A \beta$ deposits in older non-demented individuals with cognitive decline are indicative of preclinical Alzheimer's disease. Neuropsychologia, 2008. 46(6): p. 1688-1697.

356. Corder, E.H., et al., Gene dose of apolipoprotein E type 4 allele and the risk of Alzheimer's disease in late onset families. Science, 1993. 261(5123): p. 921-923.

357. Bloom, G.S., Amyloid- $\beta$ and tau: the trigger and bullet in Alzheimer disease pathogenesis. JAMA neurology, 2014. 71(4): p. 505-508.

358. Shankar, G.M., et al., Amyloid- $\beta$ protein dimers isolated directly from Alzheimer's brains impair synaptic plasticity and memory. Nature medicine, 2008. 14(8): p. 837.

359. Zempel, $\mathrm{H}$. and E. Mandelkow, Lost after translation: missorting of Tau protein and consequences for Alzheimer disease. Trends in neurosciences, 2014. 37(12): p. 721-732.

360. Mondragón-Rodríguez, S., et al., Phosphorylation of tau protein at sites Ser 396404 is one of the earliest events in A Izheimer's disease and D own syndrome. Neuropathology and applied neurobiology, 2014. 40(2): p. 121-135.

361. Veloso, A.J., et al., Electrochemical immunosensors for effective evaluation of amyloid-beta modulators on oligomeric and fibrillar aggregation processes. Analytical chemistry, 2014. 86(10): p. 4901-4909.

362. Kumar, S.T., et al., Structure and biomedical applications of amyloid oligomer nanoparticles. ACS nano, 2014. 8(11): p. 11042-11052.

363. Veloso, A.J. and K. Kerman, Modulation of fibril formation by a beta-sheet breaker peptide ligand: an electrochemical approach. Bioelectrochemistry, 2012. 84: p. 4952. 
364. Zolezzi, J.M. and N.C. Inestrosa, Peroxisome proliferator-activated receptors and Alzheimer's disease: hitting the blood-brain barrier. Molecular neurobiology, 2013. 48(3): p. 438-451.

365. Navarro Garrido, V., et al., Microglia in Alzheimer's disease: activated, dysfunctional or degenerative. Frontiers in aging neuroscience, 2018. 10: p. 140.

366. Sawikr, Y., et al., Neuroinflammation in Alzheimer's disease: the preventive and therapeutic potential of polyphenolic nutraceuticals, in Advances in protein chemistry and structural biology. 2017, Elsevier. p. 33-57.

367. Davies, D.S., et al., Microglia show altered morphology and reduced arborization in human brain during aging and A Izheimer's disease. Brain Pathology, 2017. 27(6): p. 795-808.

368. Du, L., et al., Role of microglia in neurological disorders and their potentials as a therapeutic target. Molecular neurobiology, 2017. 54(10): p. 7567-7584.

369. Orre, M., et al., Isolation of glia from Alzheimer's mice reveals inflammation and dysfunction. Neurobiology of aging, 2014. 35(12): p. 2746-2760.

370. McGeer, P., et al., Reactive microglia are positive for HLA-DR in the substantia nigra of Parkinson's and Alzheimer's disease brains. Neurology, 1988. 38(8): p. 1285-1285.

371. McGeer, P.L. and E.G. McGeer, The amyloid cascade-inflammatory hypothesis of Alzheimer disease: implications for therapy. Acta neuropathologica, 2013. 126(4): p. 479-497.

372. Bagyinszky, E., et al., Role of inflammatory molecules in the Alzheimer's disease progression and diagnosis. Journal of the neurological sciences, 2017. 376: $p$. 242-254.

373. Ray, B. and D.K. Lahiri, Neuroinflammation in Alzheimer's disease: different molecular targets and potential therapeutic agents including curcumin. Current opinion in pharmacology, 2009. 9(4): p. 434-444.

374. Mormino, E., et al., Episodic memory loss is related to hippocampal-mediated $\beta$ amyloid deposition in elderly subjects. Brain, 2008. 132(5): p. 1310-1323.

375. Pike, K.E., et al., $\beta$-amyloid imaging and memory in non-demented individuals: evidence for preclinical Alzheimer's disease. Brain, 2007. 130(11): p. 2837-2844.

376. Resnick, S., et al., Longitudinal cognitive decline is associated with fibrillar amyloid-beta measured by [11C] PiB. Neurology, 2010. 74(10): p. 807-815.

377. De Strooper, B., et al., Deficiency of presenilin-1 inhibits the normal cleavage of amyloid precursor protein. Nature, 1998. 391(6665): p. 387. 
378. Vassar, R., et al., $\beta$-Secretase cleavage of Alzheimer's amyloid precursor protein by the transmembrane aspartic protease BACE. science, 1999. 286(5440): p. 735741.

379. Cole, S.L. and R. Vassar, The role of amyloid precursor protein processing by BACE1, the $\beta$-secretase, in Alzheimer disease pathophysiology. Journal of Biological Chemistry, 2008. 283(44): p. 29621-29625.

380. Lee, M. and R. Jayant, Penetration of the blood-brain barrier by peripheral neuropeptides: new approaches to enhancing transport and endogenous expression. Cell and tissue research, 2018: p. 1-7.

381. Wilhelm, I., C. Fazakas, and I.A. Krizbai, In vitro models of the blood-brain barrier. Acta Neurobiol Exp (Wars), 2011. 71(1): p. 113-28.

382. Tiwari S, Atluri V, Yndart A, Kaushik A, Nair M, Alzheimer' disease: Pathogenesis, Diagnostics and Therapeutics. International Journal of Nanomedicine, 2019, 14, doi: $10.2147 / / J N . S 200490$ 


\section{VITA}

\section{SNEHAM TIWARI}

Born, India

\begin{tabular}{|c|c|}
\hline 2006-2009 & $\begin{array}{l}\text { Isabella Thoburn College } \\
\text { Department of Biology, } \\
\text { India } \\
\text { B.S. in Life Sciences }\end{array}$ \\
\hline 2009-2011 & $\begin{array}{l}\text { VIT University } \\
\text { School of Biosciences and Technology } \\
\text { Vellore, India } \\
\text { M.S. in Applied Microbiology }\end{array}$ \\
\hline 2014-2019 & $\begin{array}{l}\text { Florida International University } \\
\text { Herbert Wertheim College of Medicine } \\
\text { Department of Immunology and Nano- } \\
\text { medicine } \\
\text { Miami, FL. } \\
\text { Ph.D. in Biomedical Sciences }\end{array}$ \\
\hline
\end{tabular}

\section{PUBLICATIONS AND PRESENTATIONS}

Nair M, Tiwari S, Yndart A. Compositions and Methods for Treating HIV-Associated Neurocognitive disorders. Pub. No. US 1018/0256720 A1, Sep 13, (2018) (Patent)

Tiwari S, Atluri V, Kaushik A, Nair M. Alzheimer's disease: Pathogenesis, diagnostics and Therapeutics. Communicated, International Journal Of Nanomedicine, (2019).(accepted)

Tiwari S, Atluri V, Yndart A, Jayant RD, Kaushik A, Geiger JD, Nair M. Withaferin A suppresses Beta amyloid in APP expressing cells: Studies for Tat and Cocaine associated neurological dysfunctions. Frontiers in Aging Neuroscience. (2018) Sep 27;10:291.

Tiwari S, Lapierre J, Ojha CR, Martins K, Parira T, Dutta RK, Caobi A, Garbinski L, Ceyhan Y, Esteban-Lopez M, El-Hage N. Signaling pathways and therapeutic perspectives related to environmental factors associated with Multiple Sclerosis. Journal of Neuroscience Research. (2018)

Jayant RD, Tiwari S, Atluri V, Kaushik A, Tomitaka A, Yndart A, Colon-Perez L, Febo M, Nair M. Multifunctional Nanotherapeutics for the Treatment of neuroAIDS in Drug Abusers. Scientific Reports, volume 8, Article number: 12991 (2018).

Kaushik A, Vashist A, Shah, P, Tiwari S, Jayant R, Nair M. Scale-up and Current Clinical Trials for Nanogels in TherapeuticsRSC Smart Materials. 283-289, (2017). 
Jayant RD, Joshi A, Kaushik A, Tiwari S, Chaudhari R, Srivastava R, Nair M. Nanogels for Gene delivery, RSC Publishers, 30, 128, (2017).

Pawitwar SS, Dhar S, Tiwari S, Ojha CR, Lapierre J, Martins K, Rodzinski A, Parira T, Paudel I, Li J, Dutta RK, Silva MR, Kaushik A, El-Hage N. Overview on the Current Status of Zika Virus Pathogenesis and Animal Related Research, Journal of Neuroimmune Pharmacology, (2017) 12 (3), 371-388.

Kaushik A, Tiwari S, Jayant RD, Vashist A, Nikkhah-Moshaie R, El-Hage N, Nair M. Electrochemical Biosensors for Early Stage Zika Diagnostics. Trends in biotechnology, (2017) 35 (4), 308-317.

Kaushik A, Jayant RD, Tiwari S, Vashist A, Nair M. Nano-biosensors to detect betaamyloid for Alzheimer's disease management. Biosens Bioelectron. (2016) Jun 15; 80: 273-87.

Kaushik A, Shah P, Vabbina PK, Jayant RD, Tiwari S, Vashist A, Yndart A, Nair M. A label-free electrochemical immunosensor for A $\beta$ detection. Anal. Methods, (2016) 8, 61156120.

Kaushik A, Tiwari S, Jayant RD, Marty A, Nair M. Towards detection and diagnosis of Ebola virus disease at point-of-care. Biosens Bioelectron 75, 254-72. Epub (2015).

Tiwari S, Nair M. Molecular Dynamics of Amyloid- $\beta$ Protein Binding with the Potential Neuroprotectant, Withaferin A 5th Scientific Symposium, Society of Personalized NanoMedicine, FIU Nov 2018

Tiwari S, Jayant RD, Yndart A, Kaushik A, Nair M. Withaferin A suppresses Beta amyloid in APP expressing cells: Studies for HIV associated neurocognitive dysfunctions. Annual Biocomparative Immunolgy conference, FIU Mar 2018

Tiwari S, Yndart A, Atluri V, Jayant RD, Kaushik A, Tomitaka T, Nair M. Neuroprotective role of Withaferin A: Studies towards neurocognitive disorders. 24th Scientific Conference of the Society on Neuroimmune Pharmacology, 4-10 April 2018.

Tiwari S, Yndart A, Atluri V, Jayant RD, Kaushik A, Tomitaka A, Nair M. Withaferin A suppresses Beta amyloid in APP expressing cells: Studies for Alzheimer's disease. 4th Scientific Symposium of Society for Personalized Nano-Medicine. 2-3 Nov 2017

Tiwari S, Nair M. Withaferin A suppresses Beta amyloid in APP expressing cells: Studies for HIV and Cocaine associated neurocognitive dysfunctions. 23rd Scientific Conference of the Society on Neuroimmune Pharmacology, Philadelphia, April 2017

Tiwari S, Jayant RD, Yndart A, Kaushik A, Nair M. Withaferin A suppresses Beta amyloid in APP expressing cells: Studies for neurocognitive dysfunctions. Nanoflorida, 10th Annual Nanoscience Technology Symposium, 23-24 Sep 2017 